\title{
In Search of the Most Stable Molecular Configuration of Heptakis(2,6-O-dimethyl)- $\beta$-cyclodextrin and Its Complex with Mianserin: A Comparison of the B3LYP-GD2 and M062X-GD3 Results
}

\author{
Anna Ignaczak*, Łukasz Orszański \\ Theoretical and Structural Chemistry Group, Department of Physical Chemistry, Faculty of Chemistry, \\ University of Lodz, Pomorska 163/165, 90-236 Lodz, Poland \\ * Email: anna.ignaczak@chemia.uni.lodz.pl
}

\section{Electronic Supporting Information}




\section{Table of Contents:}

Figure S1 - Flow chart of the procedure applied to find the low energy structures of DM- $\beta-C D$

Figure S2 - Initial geometry of DM- $\beta-\mathrm{CD}$, three torsion angles varied in each glucose unit and atom numbering S4

Procedure - Detailed description of the conformational search performed for DM- $\beta$-CD .................................S5

Figure S3 - Flow chart of the procedure applied to find the low energy structures of MIA:DM- $\beta-C D$.................S6

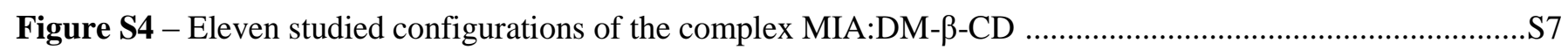

Table S1 - Total energies, enthalpies and Gibbs energies of various structures of DM- $\beta$-CD from the B3LYP-GD2 calculations

Table S2 - Total energies for selected DM- $\beta$-CD structures from the single point calculations performed with other DFT methods

Table S3 - Relative energies for selected DM- $\beta-C D$ structures from the single point calculations with other DFT methods

Table S4 - Total energies, enthalpies and Gibbs energies of various DM- $\beta$-CD structures obtained from the M062X-GD3 calculations

Figure S5 - Comparison of selected conformers of DM- $\beta$-CD optimized with the methods B3LYP-GD2, M062X-GD3 and $\omega$ B97XD

Figure S6 - IR spectra obtained for different structures with the same method

Figure S7 - Comparison of DM- $\beta-C D$ structures optimized with the 6-31G(d,p) and 6-31++G(d,p) basis sets ....S14

Figure S8 - Comparison of the B3LYP-GD2 and M062X-GD3 relative energies and Gibbs energies for DM- $\beta$-CD structures

Table S5 $-{ }^{1} \mathrm{H}$ and ${ }^{13} \mathrm{C}$ NMR chemical shifts for individual atoms in the W1 (B3LYP) and BOYFOK03 (M062X) conformers of DM- $\beta$-CD

Figure S9 $-{ }^{1} \mathrm{H}$ and ${ }^{13} \mathrm{C}$ NMR chemical shifts for selected atoms in various conformers of DM- $\beta$-CD in water ..S21

Figure S10 - The linear regression between the calculated and experimental NMR chemical shifts

Table S6 - Total energies of MIA and MIA:DM- $\beta-C D$ and complexation energies obtained from the B3LYP-GD2/6-31G(d,p) and M062X-GD3/6-31G(d,p) optimizations in water

Table S7 - Total energies of MIA and MIA:DM- $\beta-C D$ and complexation energies obtained from the single point calculations with the $6-31++G(d, p)$ basis set

Table S8 $-{ }^{1} \mathrm{H}$ and ${ }^{13} \mathrm{C}$ NMR chemical shifts for individual atoms in the CR1 (B3LYP) and NR1 (M062X) configurations of MIA:DM- $\beta-C D$

Table S9 - Atom coordinates for the DM- $\beta$-CD conformers V1 and W1 - method B3LYP-GD2/6-31G(d,p).......S29

Table S10 - Atom coordinates for the DM- $\beta$-CD conformers ZULQAY and BOYFOK03 - method M062X-GD3/6-31G(d,p)).

Table S11 - Atom coordinates for the DM- $\beta$-CD conformers ZULQAY and BOYFOK03 - method M062X-GD3/6-31++G(d,p).

Table S12 - Atom coordinates for the MIA:DM- $\beta$-CD complexes in water - method B3LYP-GD2/6-31G(d,p)).S41

Table S13 - Atom coordinates for the MIA:DM- $\beta$-CD complexes in water - method M062X-GD3/6-31G(d,p) S58

References ...S75 


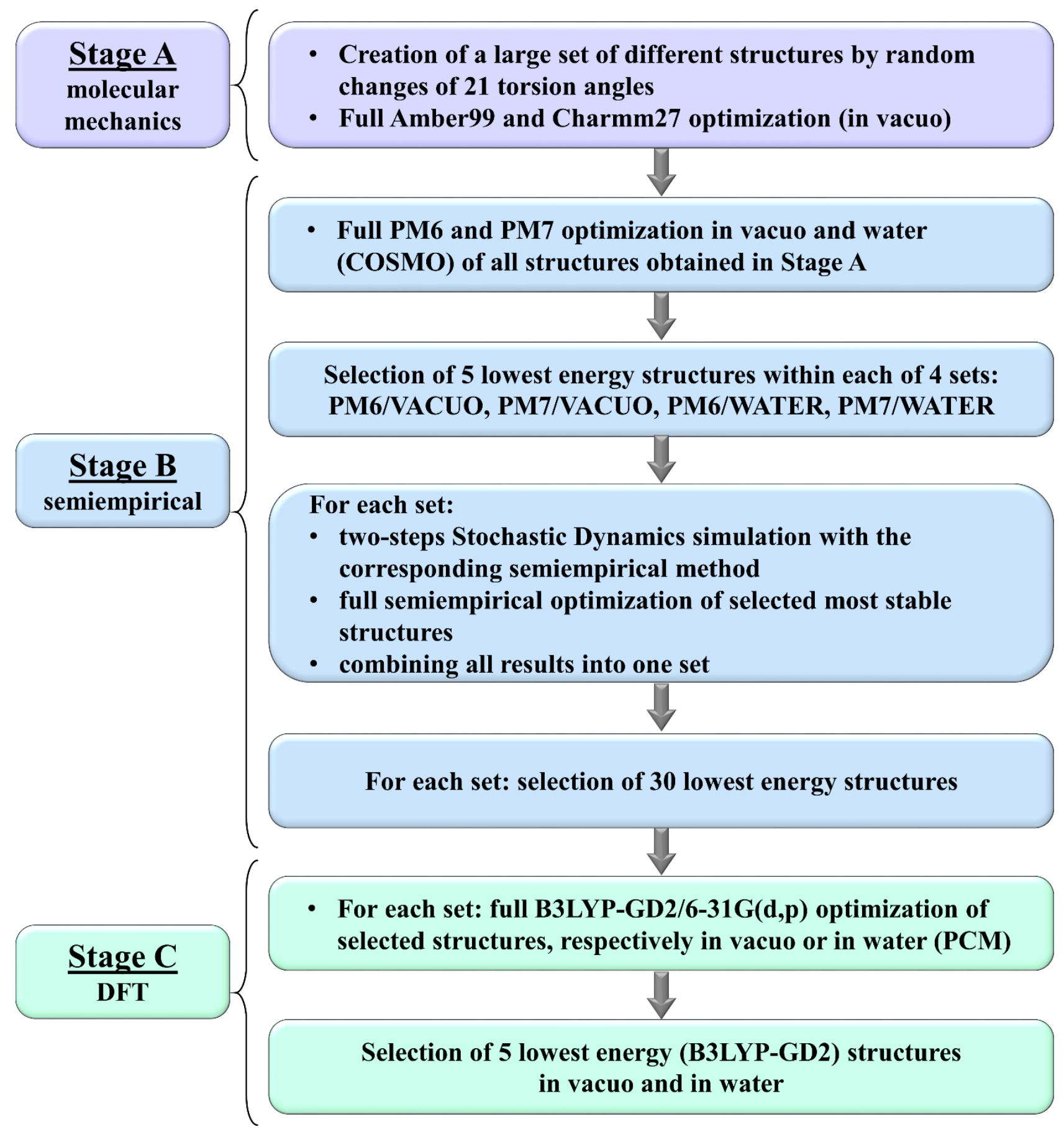

Figure S1. Flow chart of the procedure applied to find the lowest energy structures of the molecule DM- $\beta-C D$. 


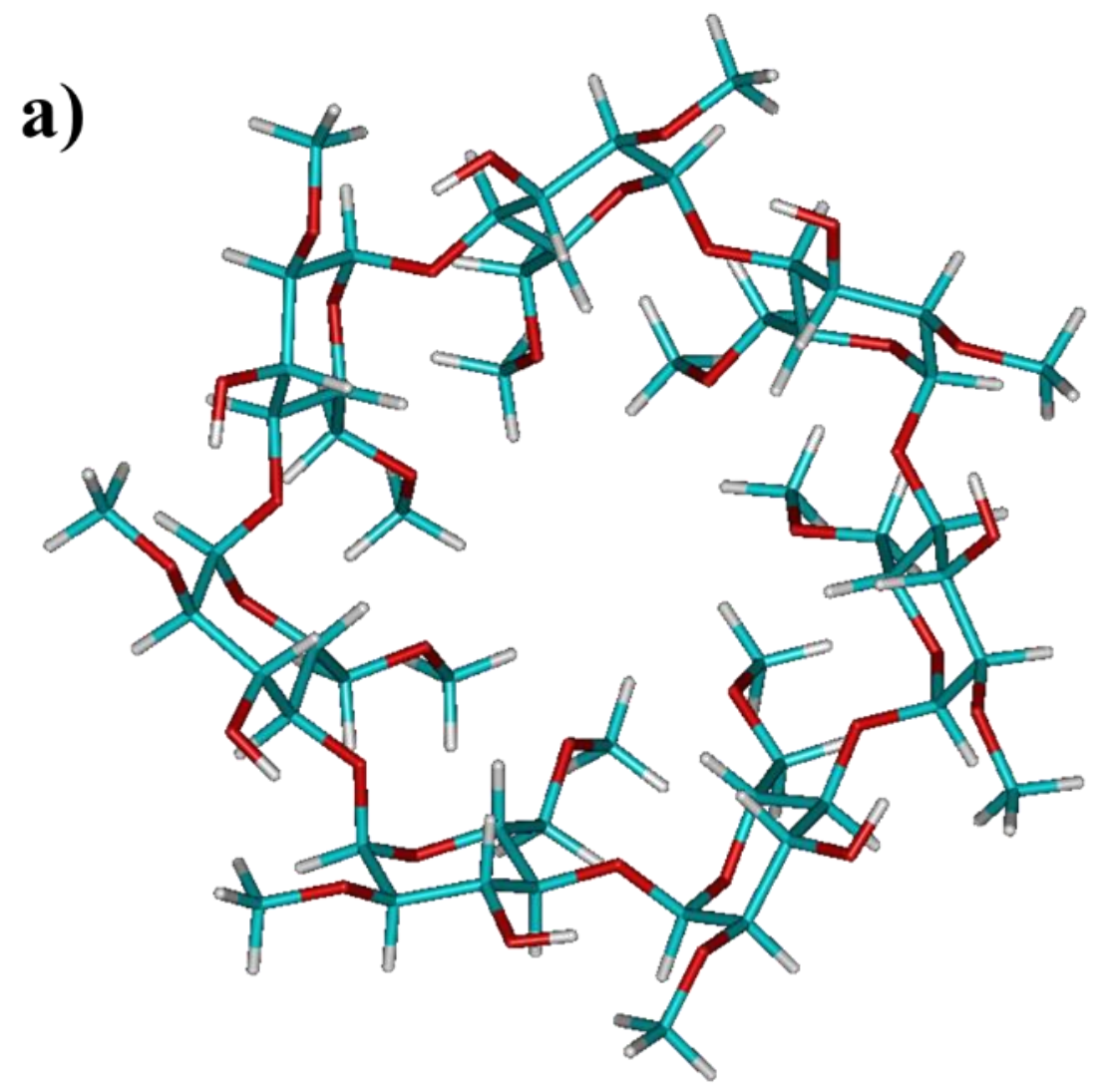

b)

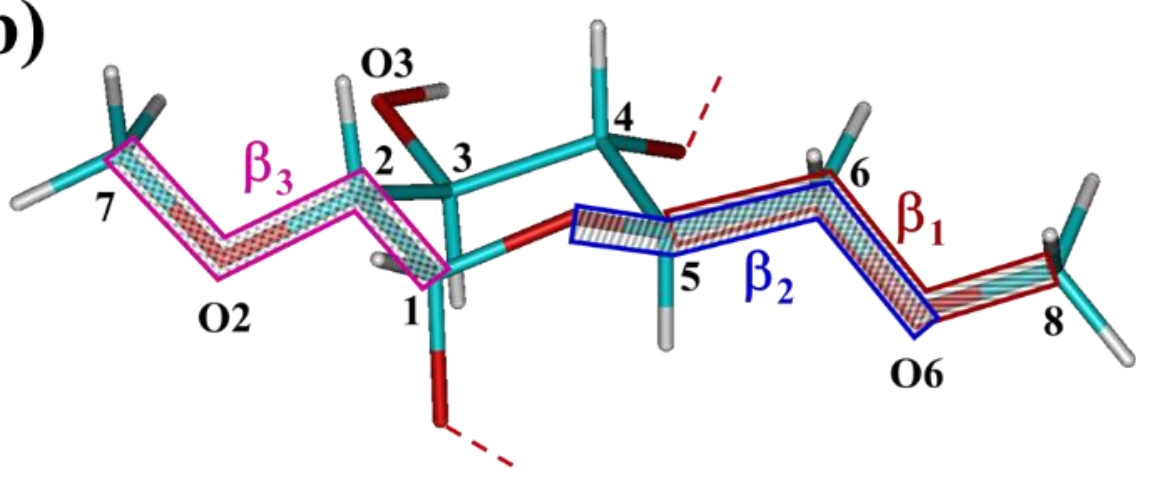

Figure S2. a) Geometry of the DM- $\beta-C D$ molecule used as an initial structure for construction of various conformers (view from the wider side of the cone), b) three torsion angles modified in each glucose unit in the procedures of construction of various conformers and the atom numbering. 


\section{Procedure}

Detailed description of the conformational search performed for DM- $\beta-C D$

Stage A: The initial structure was built in the Hyperchem program ${ }^{1}$ and it had the regular geometry shown in Fig. S2a. In each glucose unit of this structure were selected three torsion angles (Fig. $\mathrm{S} 2 \mathrm{~b}$ ), giving in total 21 torsion angles. The test conformers were produced using the molecular mechanics force fields Amber99 and Charmm27 and the Conformational Search module available in the program Hyperchem program. In the search each new conformer was created by random, simultaneous variation, in the range of $0-180^{\circ}$, of up to 3 torsion angles from the 21 torsion angles selected. The lowest energy conformers found with each force field were stored, giving in total 1722 structures which served as initial geometries in the next stage.

Stage B: All conformers were afterwards optimized in vacuo and in water using two different semiempirical methods: PM6 and PM7 available in the program MOPAC. ${ }^{2}$ In the semiempirical calculations the solvent was described with the COSMO model, ${ }^{3}$ using the values of 78.39 and 1.3 $\AA$ for the dielectric constant and the effective radius, respectively. From all geometries obtained were selected 10 structures corresponding to the lowest heats of formation in vacuo and 10 structures in water; both sets included 5 structures from the PM6 calculations and 5 structures from the PM7 calculations. For each conformer, further examination was performed using the Molecular Dynamics Conformational Search module available in the program Gabedit ${ }^{4}$ in conjunction with the MOPAC program (PM6 and PM7 methods). The goal of this stage was to allow the molecule to adopt the geometry that was more stable in terms of energy. The simulations were performed using the Stochastic Dynamics via the Verlet algorithm. The conformational space was explored at $\mathrm{T}=1000 \mathrm{~K}$ during the period of $20 \mathrm{ps}$ with the time-step $1 \mathrm{fs}$. In each simulation, from the calculated trajectory 40 lowest energy conformations were selected and subsequently optimized at the corresponding semiempirical level. From all conformers collected within each set (PM6/VACUO, PM6/WATER, PM7/VACUO, PM7/WATER) were selected 3 most stable structures, for which the simulation was repeated and the lowest energy conformers were stored. The conformers selected in the simulations were merged with these obtained earlier with the same semiempirical method (PM6 or PM7, each in vacuo or in water), which led to creation of four sets of structures. Within each set were selected the 30 lowest energy conformers, which were used as initial geometries in the DFT calculations.

Stage C: All structures obtained in Stage B were fully optimized in the Gaussian09 program ${ }^{5}$ using the 6-31G(d,p) basis set and the B3LYP-GD2 method. ${ }^{6,7}$ For the 60 structures selected within the two sets PM6/VACUO and PM7/VACUO the DFT calculations were performed in vacuo, while for the 60 structures selected within the two sets PM6/WATER and PM7/WATER - in water. In the DFT calculations the solvent was described with the Polarizable Continuum Model (PCM). ${ }^{8}$ 


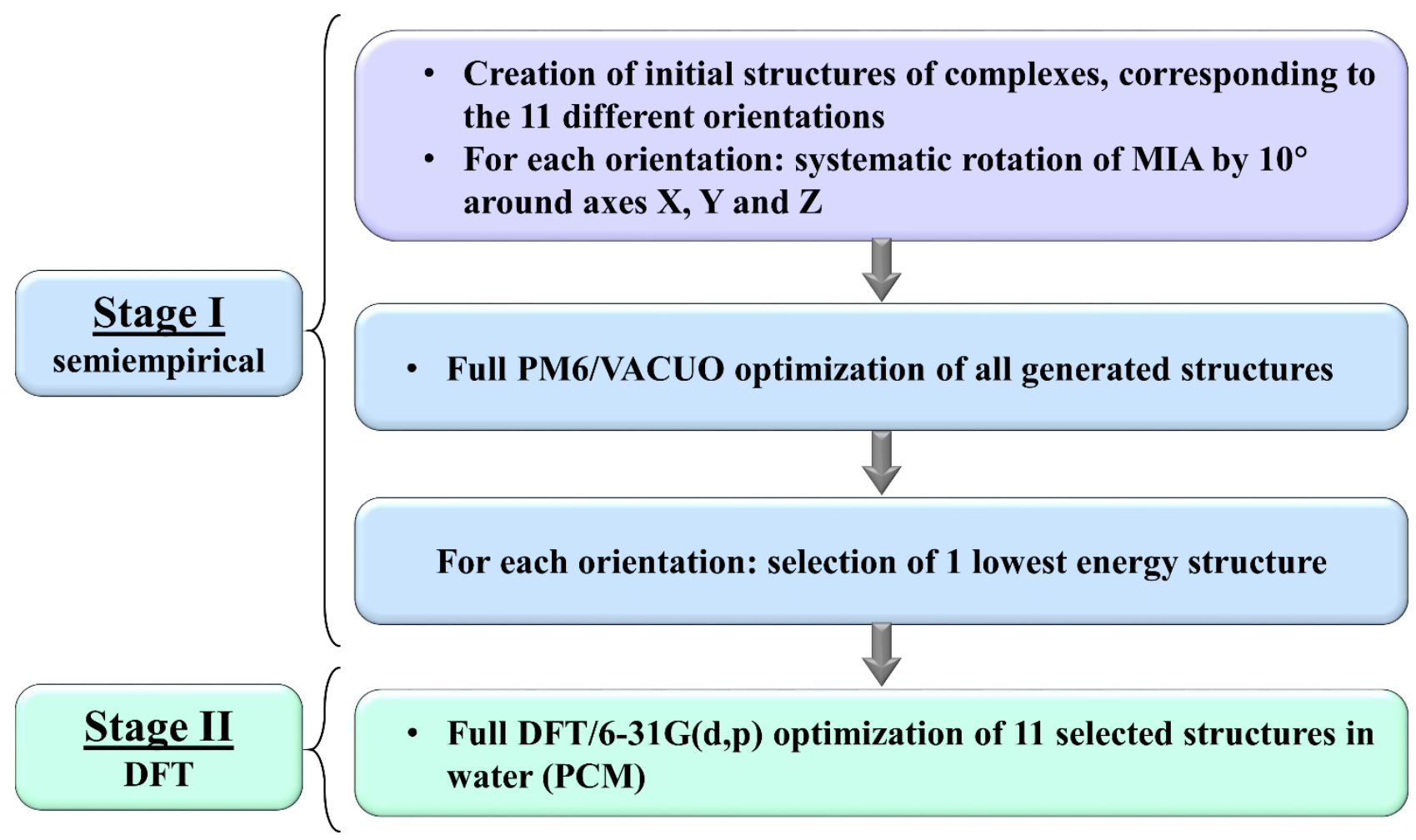

Figure S3. Flow chart of the procedure applied to find the lowest energy structures of the complex MIA:DM- $\beta-C D$. 


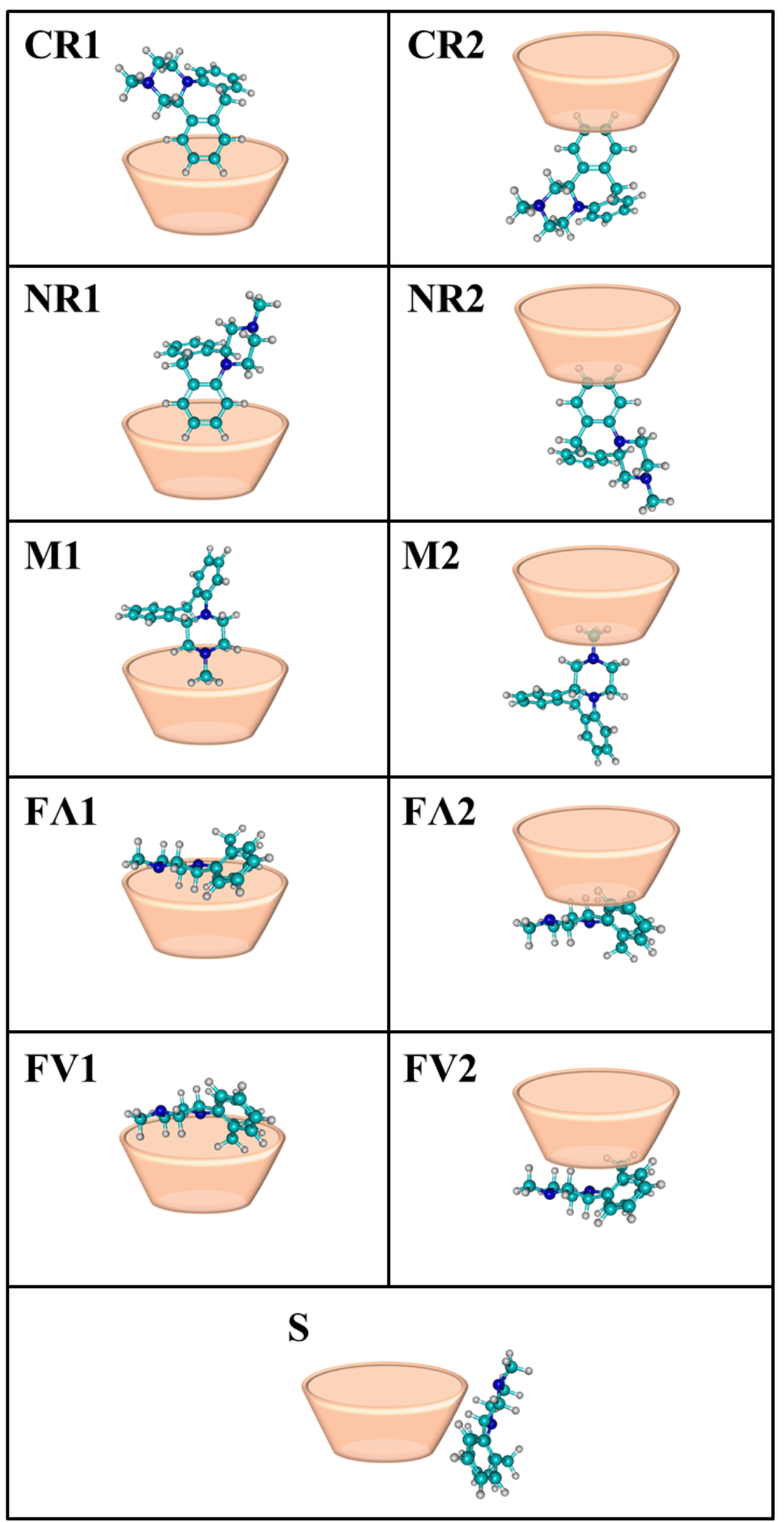

Figure S4. Eleven configurations of the complex MIA:DM- $\beta-C D(1: 1)$ considered in the calculations. CR, NR and M indicate the fragments of the MIA molecule (Chart $1 \mathrm{~b}$ in the main article) inserted in the interior cavity of DM- $\beta-\mathrm{CD}, \mathrm{F} \Lambda$ and FV - two different "flat" orientations, numbers 1,2 and the label $\mathrm{S}$ - different sides of the cyclodextrin cone. 
Table S1. The total electronic energies $E$, enthalpies $H$ and corrected Gibbs energies $G_{\text {corr }}$ obtained from the B3LYP-GD2/6-31G(d,p) calculations performed for the experimental structures of DM- $\beta$-CD (ZULQAY, CEQCUW, BOYFOK03, BOYFOK04, PABNEM; single point calculations for the original structures and after their optimization) and for the lowest energy conformers in vacuo (V1-V5) and in water (W1-W5), found from the conformational search. In the last column are given the electronic energies $E$ obtained from the single point (SP) calculations performed with the 6-31++G(d,p) basis set for the optimized structures. All values are in hartree.

\begin{tabular}{|c|c|c|c|c|c|c|}
\hline Structure & $\begin{array}{c}\text { Single } \\
\text { point } \\
E \\
\end{array}$ & $\begin{array}{c}\text { Optimized } \\
E\end{array}$ & $\begin{array}{c}\text { Optimized } \\
H\end{array}$ & $\begin{array}{c}\text { Optimized } \\
G\end{array}$ & $\begin{array}{c}\text { Optimized } \\
G_{\text {corr }}\end{array}$ & $\begin{array}{c}\text { SP } \\
6-31++G(d, p) \\
E \\
\end{array}$ \\
\hline \multicolumn{7}{|c|}{ in VACUO } \\
\hline ZULQAY & -4825.617085 & -4826.001307 & -4824.292511 & -4824.525224 & -4824.503101 & -4826.170912 \\
\hline CEQCUW & -4824.340915 & -4825.990171 & -4824.281332 & -4824.512566 & -4824.491477 & -4826.160026 \\
\hline BOYFOK03 & -4825.678624 & -4825.999707 & -4824.290515 & -4824.520273 & -4824.499660 & -4826.167843 \\
\hline BOYFOK04 & -4824.318150 & -4825.996993 & -4824.287142 & -4824.513869 & -4824.494785 & -4826.165639 \\
\hline PABNEM & -4824.661886 & -4826.002305 & -4824.293503 & -4824.524125 & -4824.503220 & -4826.171156 \\
\hline V1 & & -4826.005732 & -4824.295165 & -4824.518858 & -4824.500920 & -4826.171643 \\
\hline $\mathbf{V 2}$ & & -4826.005230 & -4824.294261 & -4824.519012 & -4824.500011 & -4826.168882 \\
\hline $\mathbf{V 3}$ & & -4826.003686 & -4824.293172 & -4824.519233 & -4824.499681 & -4826.167411 \\
\hline V4 & & -4826.003688 & -4824.292020 & -4824.512959 & -4824.496391 & -4826.161819 \\
\hline V5 & & -4826.003502 & -4824.292090 & -4824.517113 & -4824.497719 & -4826.163592 \\
\hline \multicolumn{7}{|c|}{ in WATER (PCM) } \\
\hline ZULQAY & -4825.665558 & -4826.042086 & -4824.335457 & -4824.568213 & -4824.534616 & -4826.221417 \\
\hline CEQCUW & -4824.406142 & -4826.033616 & -4824.327440 & -4824.561236 & -4824.527433 & -4826.214908 \\
\hline BOYFOK03 & -4825.737045 & -4826.045585 & -4824.338372 & -4824.570357 & -4824.537027 & -4826.224433 \\
\hline BOYFOK04 & -4824.385492 & -4826.041185 & -4824.333842 & -4824.562841 & -4824.531282 & -4826.220130 \\
\hline PABNEM & -4824.715739 & -4826.040870 & -4824.334607 & -4824.567460 & -4824.533894 & -4826.219507 \\
\hline W1 & & -4826.050254 & -4824.341034 & -4824.563364 & -4824.534257 & -4826.227305 \\
\hline W2 & & -4826.049687 & -4824.340968 & -4824.565247 & -4824.535201 & -4826.226859 \\
\hline W3 & & -4826.048893 & -4824.340023 & -4824.564005 & -4824.534213 & -4826.224549 \\
\hline W4 & & -4826.047957 & -4824.338297 & -4824.559989 & -4824.530895 & -4826.223671 \\
\hline W5 & & -4826.046416 & -4824.336733 & -4824.558734 & -4824.529630 & -4826.223056 \\
\hline
\end{tabular}


Table S2. The total electronic energies $E$ obtained from the single point calculations performed with several other DFT methods and the 6-31G(d,p) basis set for the DM- $\beta$-CD structures optimized with the B3LYP-GD2/6-31G(d,p) method. All values are in hartree.

\begin{tabular}{ccccccc}
\hline Structure & M05-GD3 & M06-GD3 & M062X-GD3 & WB97XD & mPW1PW91 & M11 \\
\hline ZULQAY & -4823.053737 & -4823.023173 & -4823.793952 & -4824.317152 & -4824.481434 & -4823.471934 \\
CEQCUW & -4823.042455 & -4823.012967 & -4823.781011 & -4824.304906 & -4824.464448 & -4823.455722 \\
BOYFOK03 & -4823.049564 & -4823.025387 & -4823.790332 & -4824.315399 & -4824.469298 & -4823.465195 \\
BOYFOK04 & -4823.045560 & -4823.018277 & -4823.784139 & -4824.309597 & -4824.462025 & -4823.458134 \\
PABNEM & -4823.054008 & -4823.022882 & -4823.793160 & -4824.317011 & -4824.480515 & -4823.471866 \\
V1 & -4823.050061 & -4823.020972 & -4823.788802 & -4824.315282 & -4824.464694 & -4823.463261 \\
V2 & -4823.046317 & -4823.019080 & -4823.788886 & -4824.312591 & -4824.460037 & -4823.466832 \\
V3 & -4823.045752 & -4823.017509 & -4823.789023 & -4824.312293 & -4824.462066 & -4823.466990 \\
V4 & -4823.036804 & -4823.024979 & -4823.782714 & -4824.309393 & -4824.421864 & -4823.455157 \\
V5 & -4823.043211 & -4823.017440 & -4823.784153 & -4824.310307 & -4824.452413 & -4823.460063 \\
\hline & & & in WATER (PCM) & & \\
\hline ZULQAY & -4823.093929 & -4823.063286 & -4823.835604 & -4824.359910 & -4824.522926 & -4823.516462 \\
CEQCUW & -4823.087391 & -4823.055930 & -4823.827084 & -4824.351570 & -4824.515744 & -4823.505944 \\
BOYFOK03 & -4823.096258 & -4823.070885 & -4823.838428 & -4824.363802 & -4824.519056 & -4823.517566 \\
BOYFOK04 & -4823.091122 & -4823.064439 & -4823.830848 & -4824.357325 & -4824.509589 & -4823.508227 \\
PABNEM & -4823.093177 & -4823.061116 & -4823.833363 & -4824.357984 & -4824.520907 & -4823.514940 \\
W1 & -4823.093172 & -4823.068445 & -4823.834813 & -4824.362721 & -4824.508782 & -4823.510844 \\
W2 & -4823.093424 & -4823.067069 & -4823.834212 & -4824.362162 & -4824.509683 & -4823.510452 \\
W3 & -4823.093320 & -4823.067626 & -4823.833037 & -4824.361121 & -4824.507820 & -4823.508814 \\
W4 & -4823.088333 & -4823.065721 & -4823.832024 & -4824.359293 & -4824.502099 & -4823.508596 \\
W5 & -4823.087409 & -4823.061778 & -4823.827021 & -4824.357252 & -4824.503056 & -4823.501273 \\
\hline & & & & & & \\
\hline
\end{tabular}


Table S3. The relative energies $\Delta E[\mathrm{kcal} / \mathrm{mol}]$ obtained from the single point (SP) calculations performed using several other DFT methods and the 6-31G(d,p) basis set, for the DM- $\beta-C D$ structures optimized with the B3LYP-GD2 method. The $\Delta E$ values are always calculated with respect to the lowest energy conformer indicated by a given method.

\begin{tabular}{ccccccc}
\hline Structure & M05-GD3 & M06-GD3 & M062X-GD3 & $\omega$ WB97XD & mPW1PW91 & M11 \\
\hline & \multicolumn{6}{c}{ in VACUO } \\
\hline ZULQAY & 0.17 & 1.39 & 0.00 & 0.00 & 0.00 & 0.00 \\
CEQCUW & 7.25 & 7.79 & 8.12 & 7.68 & 10.66 & 10.17 \\
BOYFOK03 & 2.79 & 0.00 & 2.27 & 1.10 & 7.62 & 4.23 \\
BOYFOK04 & 5.30 & 4.46 & 6.16 & 4.74 & 12.18 & 8.66 \\
PABNEM & 0.00 & 1.57 & 0.50 & 0.09 & 0.58 & 0.04 \\
V1 & 2.48 & 2.77 & 3.23 & 1.17 & 10.50 & 5.44 \\
V2 & 4.83 & 3.96 & 3.18 & 2.86 & 13.43 & 3.20 \\
V3 & 5.18 & 4.94 & 3.09 & 3.05 & 12.15 & 3.10 \\
V4 & 10.80 & 0.26 & 7.05 & 4.87 & 37.38 & 10.53 \\
V5 & 6.78 & 4.99 & 6.15 & 4.30 & 18.21 & 7.45 \\
\hline & & & in WATER (PCM) & & \\
\hline ZULQAY & 1.46 & 4.77 & 1.77 & 2.44 & 0.00 & 0.69 \\
CEQCUW & 5.56 & 9.38 & 7.12 & 7.68 & 4.51 & 7.29 \\
BOYFOK03 & 0.00 & 0.00 & 0.00 & 0.00 & 2.43 & 0.00 \\
BOYFOK04 & 3.22 & 4.05 & 4.76 & 4.06 & 8.37 & 5.86 \\
PABNEM & 1.93 & 6.13 & 3.18 & 3.65 & 1.27 & 1.65 \\
W1 & 1.94 & 1.53 & 2.27 & 0.68 & 8.88 & 4.22 \\
W2 & 1.78 & 2.39 & 2.65 & 1.03 & 8.31 & 4.46 \\
W3 & 1.84 & 2.04 & 3.38 & 1.68 & 9.48 & 5.49 \\
W4 & 4.97 & 3.24 & 4.02 & 2.83 & 13.07 & 5.63 \\
W5 & 5.55 & 5.71 & 7.16 & 4.11 & 12.47 & 10.22 \\
\hline & & & & & &
\end{tabular}


Table S4. The total electronic energies $E$, enthalpies $H$ and corrected Gibbs energies $G_{\text {corr }}$ of DM- $\beta$-CD obtained with the M062X-GD3/6-31G(d,p) method: from the single point calculations performed for the original experimental structures and from the M062X-GD3 re-optimization of the structures optimized with the B3LYP-GD2. In the last column are given the electronic energies $E$ obtained from the single point (SP) calculations performed with the $6-31++\mathrm{G}(\mathrm{d}, \mathrm{p})$ basis set for the optimized structures. All values are in hartree.

\begin{tabular}{|c|c|c|c|c|c|c|}
\hline Structure & $\begin{array}{c}\text { Single } \\
\text { point } \\
E\end{array}$ & $\begin{array}{c}\text { Optimized } \\
E\end{array}$ & $\begin{array}{c}\text { Optimized } \\
H\end{array}$ & $\begin{array}{c}\text { Optimized } \\
\qquad G\end{array}$ & $\begin{array}{c}\text { Optimized } \\
G_{\text {corr }}\end{array}$ & $\begin{array}{c}\text { SP } \\
6-31++G(d, p) \\
E \\
\end{array}$ \\
\hline \multicolumn{7}{|c|}{ in VACUO } \\
\hline ZULQAY & -4823.389601 & -4823.800060 & -4822.070092 & -4822.302710 & -4822.280333 & -4823.942002 \\
\hline CEQCUW & -4822.132292 & -4823.793097 & -4822.063048 & -4822.291878 & -4822.271529 & -4823.935096 \\
\hline BOYFOK03 & -4823.465779 & -4823.796939 & -4822.066878 & -4822.294971 & -4822.274881 & -4823.938049 \\
\hline BOYFOK04 & -4822.109973 & -4823.789994 & -4822.059647 & -4822.287794 & -4822.267637 & -4823.932270 \\
\hline PABNEM & -4822.444621 & -4823.799771 & -4822.069790 & -4822.300436 & -4822.279111 & -4823.941596 \\
\hline V1 & & -4823.794917 & -4822.064295 & -4822.289657 & -4822.270578 & -4823.935458 \\
\hline $\mathbf{V} 2$ & & -4823.795359 & -4822.064328 & -4822.289229 & -4822.269913 & -4823.933750 \\
\hline V3 & & -4823.794921 & -4822.063866 & -4822.288782 & -4822.269502 & -4823.933153 \\
\hline V4 & & -4823.789650 & -4822.059227 & -4822.279908 & -4822.263412 & -4823.927277 \\
\hline V5 & & -4823.791684 & -4822.060418 & -4822.285233 & -4822.265604 & -4823.929223 \\
\hline \multicolumn{7}{|c|}{ in WATER (PCM) } \\
\hline ZULQAY & -4823.441988 & -4823.842104 & -4822.114703 & -4822.346383 & -4822.313262 & -4823.991933 \\
\hline CEQCUW & -4822.201628 & -4823.833921 & -4822.106670 & -4822.338753 & -4822.305780 & -4823.985570 \\
\hline BOYFOK03 & -4823.528403 & -4823.844581 & -4822.116697 & -4822.346140 & -4822.313896 & -4823.993905 \\
\hline BOYFOK04 & -4822.181288 & -4823.838670 & -4822.111056 & -4822.338606 & -4822.307485 & -4823.988344 \\
\hline PABNEM & -4822.502021 & -4823.840110 & -4822.113025 & -4822.345536 & -4822.312037 & -4823.990291 \\
\hline W1 & & -4823.840641 & -4822.112514 & -4822.338050 & -4822.307429 & -4823.990322 \\
\hline W2 & & -4823.840239 & -4822.112232 & -4822.338670 & -4822.307607 & -4823.990100 \\
\hline W3 & & -4823.839681 & -4822.111671 & -4822.336153 & -4822.305937 & -4823.988730 \\
\hline W4 & & -4823.838028 & -4822.109390 & -4822.331511 & -4822.302265 & -4823.986724 \\
\hline W5 & & -4823.833373 & -4822.105102 & -4822.329521 & -4822.299215 & -4823.983184 \\
\hline
\end{tabular}


a) VACUO ZULQAY

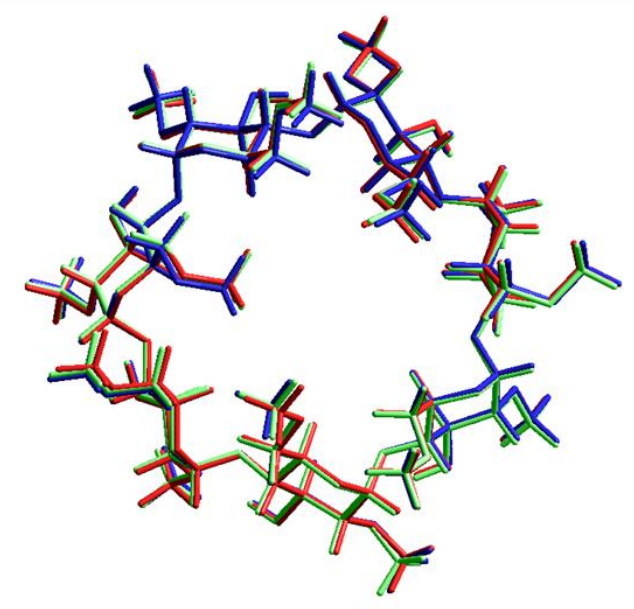

RMSD $1=0.191$

RMSD $2=0.136$

c) WATER BOYFOK03

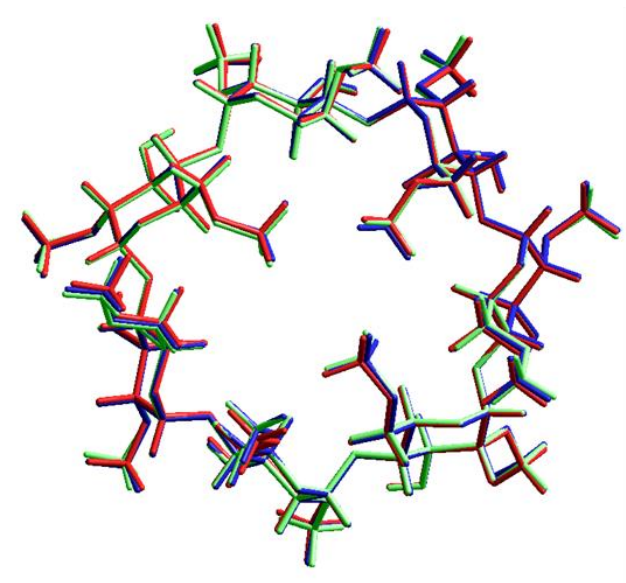

RMSD $1=0.107$

RMSD $2=0.109$ b) VACUO V1

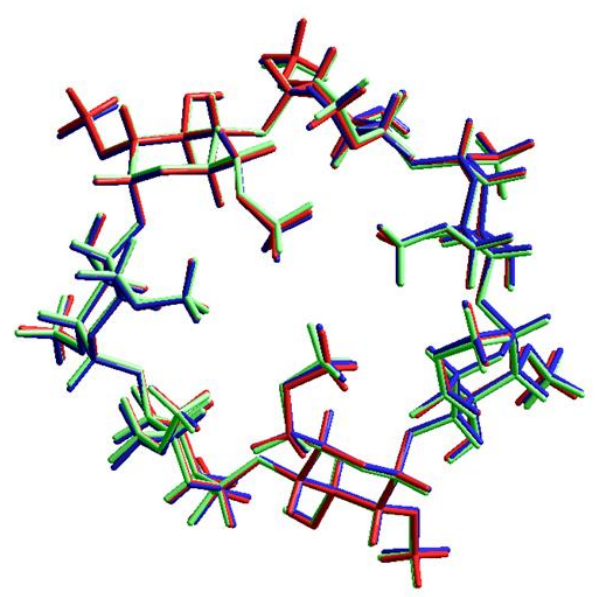

RMSD $1=0.104$

RMSD $2=0.103$ c) WATER W1

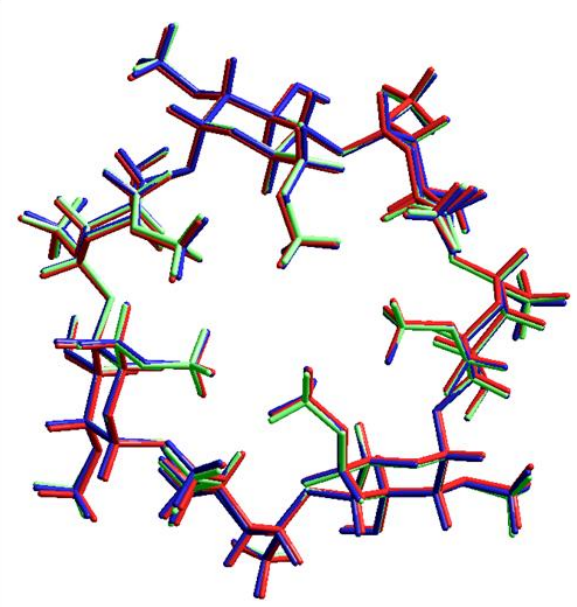

RMSD $1=0.074$

RMSD $2=0.092$

Figure S5. Comparison of the DM- $\beta$-CD structures indicated by various methods as the most stable conformers, obtained from the B3LYP-GD2/6-31G(d,p) (dark blue), M062X-GD3/6-31G(d,p) (red) and $\omega \mathrm{B} 97 \mathrm{XD} / 6-31 \mathrm{G}(\mathrm{d}, \mathrm{p})$ (green) optimizations in vacuo $(\mathrm{a}, \mathrm{b})$ and in water $(\mathrm{c}, \mathrm{d})$. For each case, the root-mean square deviations of atomic positions (in $\AA$ ) for the structures optimized with the methods M062X-GD3 (RMSD 1) and $\omega$ B97XD (RMSD 2), calculated always with respect to the structures optimized with the B3LYP-GD2 method, are also given. 

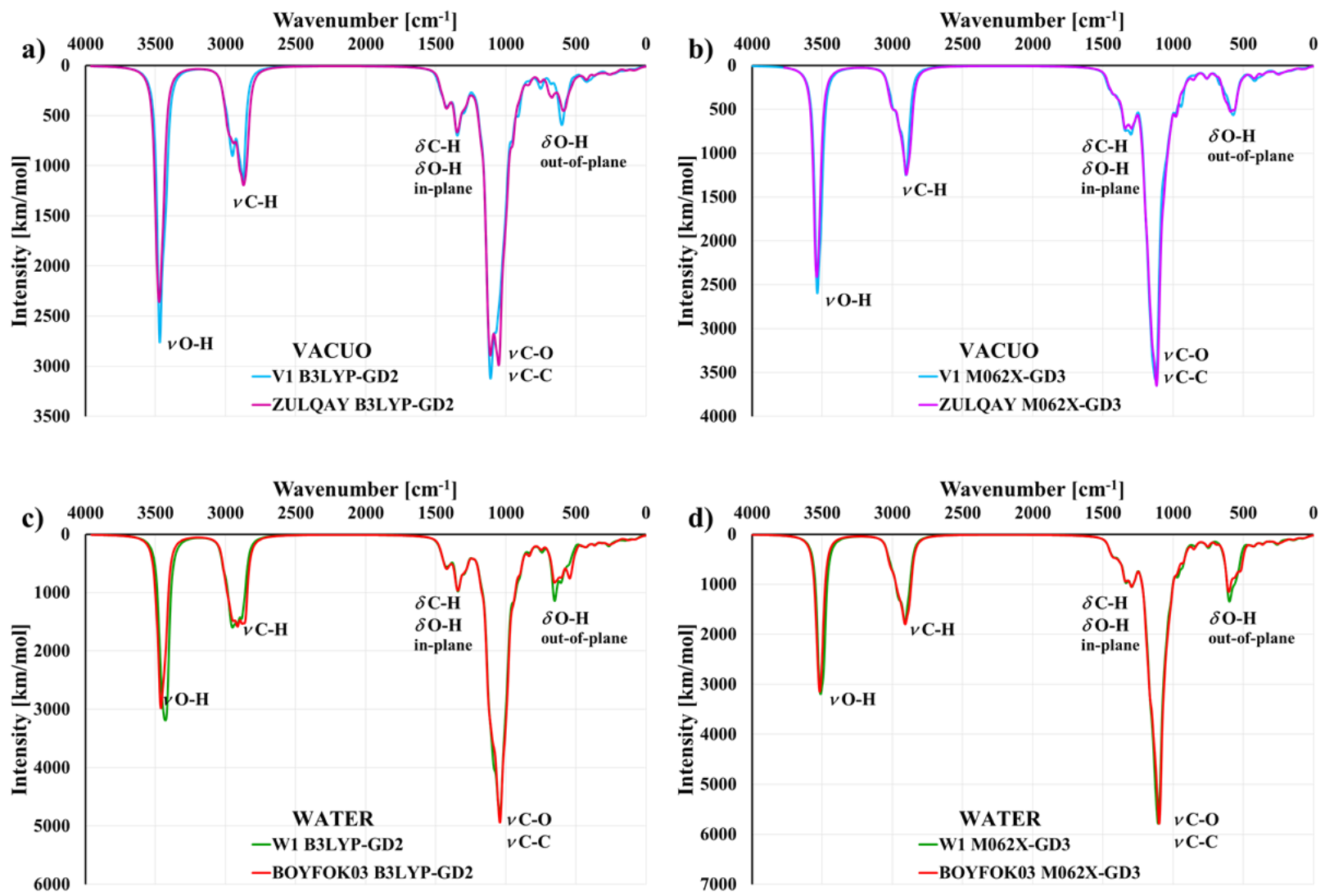

Figure S6. Comparison of IR spectra obtained from the B3LYP-GD2/6-31G(d,p) (a,c) and M062X-GD3/6-31G(d,p) (b,d) calculations for the structures of V1 and ZULQAY in vacuo (a,b) and for the structures of W1 and BOYFOK03 in water $(\mathrm{c}, \mathrm{d})$ optimized with the same DFT methods. 
a) B3LYP-GD2 V1

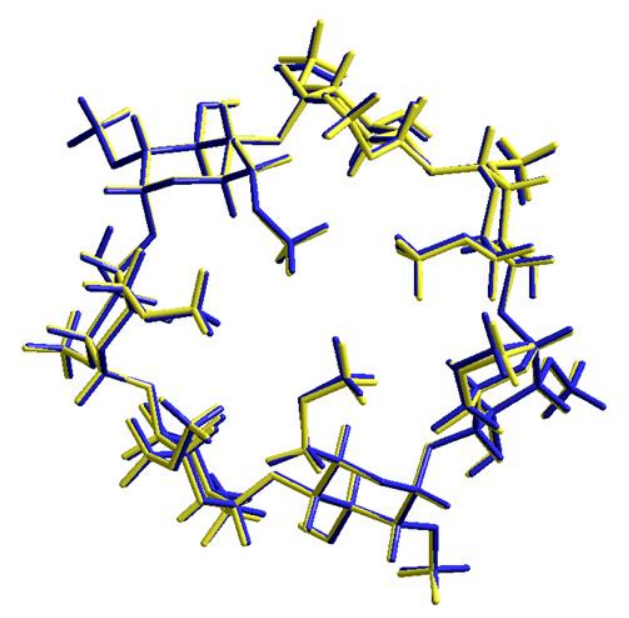

RMSD $=0.112$

c) M062X-GD3 ZULQAY

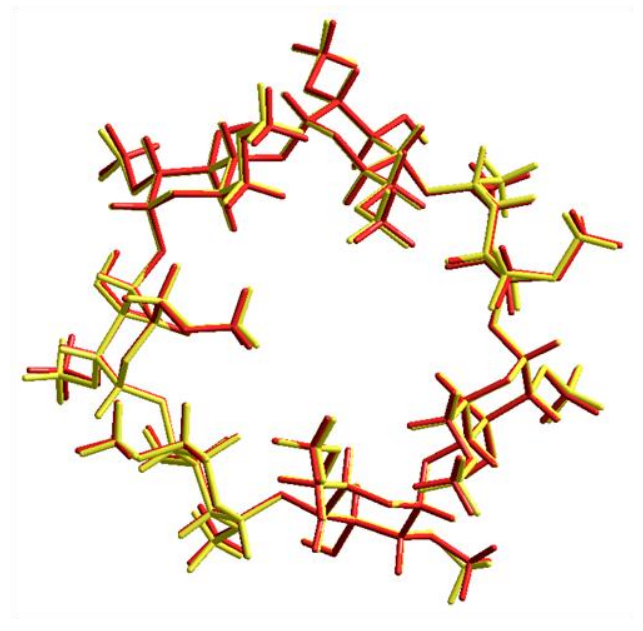

RMSD $=0.164$ b) B3LYP-GD2 W1

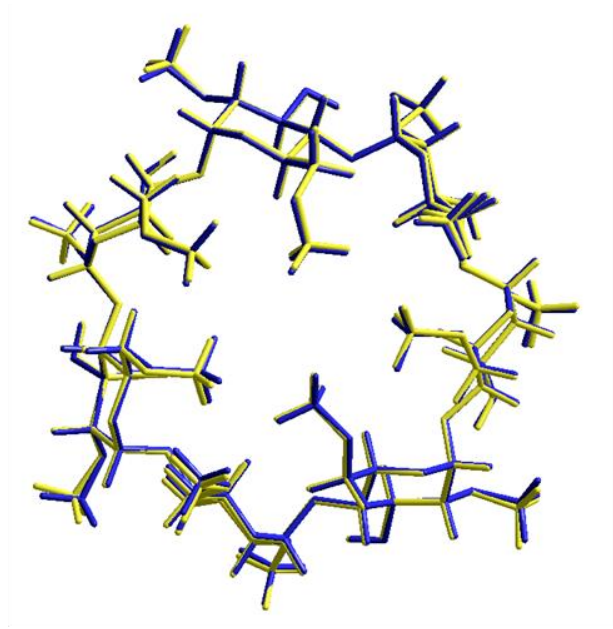

RMSD $=0.106$

\section{d) M062X-GD3 BOYFOK03}

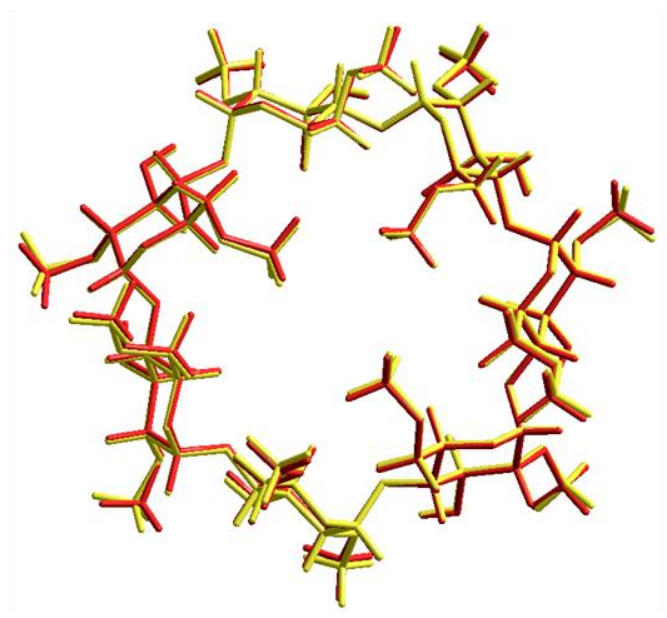

RMSD $=\mathbf{0 . 1 3 2}$

Figure S7. Comparison of the DM- $\beta$-CD structures obtained from the B3LYP-GD2/6-31G(d,p) and M062X-GD3/6-31G(d,p) optimizations (blue and red, respectively) performed in vacuo $(\mathrm{a}, \mathrm{c})$ and in water $(\mathrm{b}, \mathrm{d})$ with the structures obtained after their re-optimization with the same DFT methods and the $6-31++\mathrm{G}(\mathrm{d}, \mathrm{p})$ basis set (yellow in all cases). For each case, the root-mean square deviation (RMSD) of atomic positions (in $\AA$ ) is also given. 

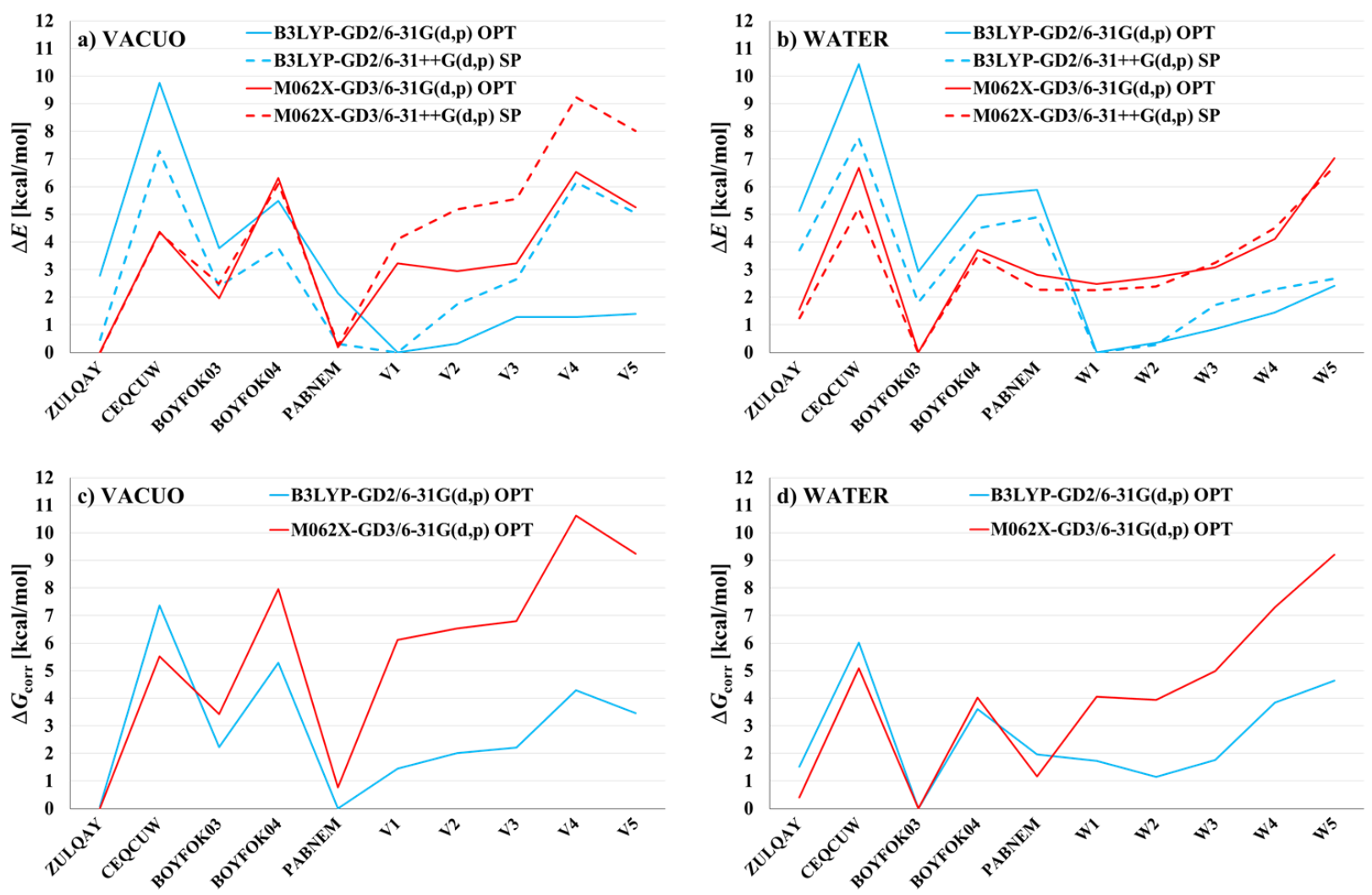

Figure S8. Comparison of the relative energies $\Delta E(\mathrm{a}, \mathrm{b})$ and corrected Gibbs energies $\Delta G_{\text {corr }}(\mathrm{c}, \mathrm{d})$ calculated with respect to the lowest energy conformer, obtained for various structures of DM- $\beta$ CD optimized (OPT) with the B3LYP-GD2/6-31G(d,p) and M062X-GD3/6-31G(d,p) methods in vacuo and water (PCM). The corresponding energy values obtained from the single point calculations (SP) performed with the 6-31++G(d,p) basis set are included in the plots (a) and (b). 
Table S5. The computed ${ }^{1} \mathrm{H}$ and ${ }^{13} \mathrm{C}$ NMR chemical shifts $\left(\delta_{\text {calc }}\right)[\mathrm{ppm}]$ for individual atoms in the most stable conformers of DM- $\beta$-CD in water indicated by the methods B3LYP-GD2 (W1) and M062X-GD3 (BOYFOK03) obtained from the B3LYP/6-31++G(d,p)//B3LYP-GD2/6-31G(d,p) and M062X/6-31++G(d,p)//M062X-GD3/6-31G(d,p) calculations, respectively. The $\delta_{\text {calc }}$ are scaled according to the procedure of Tantillo, ${ }^{9}$ using the formula $\delta=(\mathrm{I}-\sigma) /(-\mathrm{S})$, where $\sigma$ are the isotropic values obtained from DFT calculations, and I and S are the scaling factors obtained with a given method. For the B3LYP method their values in water $(\mathrm{PCM})$ are: $\mathrm{S}_{\mathrm{H}}=-1.0567, \mathrm{I}_{\mathrm{H}}$ $=31.6557, \mathrm{~S}_{\mathrm{C}}=-0.9687, \mathrm{I}_{\mathrm{C}}=189.8139 ;{ }^{10}$ the scaling factors for the M062X method are given in the main article.

\begin{tabular}{|c|c|c|c|c|c|c|}
\hline \multirow{3}{*}{$\begin{array}{l}\text { Atom } \\
\text { no. } \\
\text { in Fig. } \\
\text { S2b }\end{array}$} & \multicolumn{6}{|c|}{ a) Protons } \\
\hline & \multirow[b]{2}{*}{$\begin{array}{c}\text { Atom } \\
\text { no. in } \\
\text { Table S9 }\end{array}$} & \multicolumn{2}{|c|}{ W1 B3LYP } & \multicolumn{3}{|c|}{ BOYFOK03 M062X } \\
\hline & & Isotropic & $\delta_{\text {calc }}$ & $\begin{array}{c}\text { Atom } \\
\text { no. in } \\
\text { Table } S 10\end{array}$ & Isotropic & $\delta_{\text {calc }}$ \\
\hline $\mathrm{H}-1$ & 11 & 26.6442 & 4.74 & 14 & 27.0612 & 4.28 \\
\hline $\mathrm{H}-1$ & 33 & 26.8390 & 4.56 & 40 & 26.8244 & 4.49 \\
\hline $\mathrm{H}-1$ & 54 & 26.7572 & 4.64 & 67 & 26.7271 & 4.57 \\
\hline $\mathrm{H}-1$ & 75 & 26.6604 & 4.73 & 94 & 26.8800 & 4.44 \\
\hline $\mathrm{H}-1$ & 96 & 26.8174 & 4.58 & 121 & 26.9003 & 4.42 \\
\hline $\mathrm{H}-1$ & 117 & 26.5970 & 4.79 & 148 & 26.7061 & 4.59 \\
\hline $\mathrm{H}-1$ & 138 & 26.7238 & 4.67 & 175 & 26.3486 & 4.90 \\
\hline $\mathrm{H}-2$ & 10 & 28.4516 & 3.03 & 15 & 28.8713 & 2.72 \\
\hline $\mathrm{H}-2$ & 32 & 28.3954 & 3.09 & 41 & 28.8051 & 2.78 \\
\hline $\mathrm{H}-2$ & 53 & 28.4054 & 3.08 & 68 & 28.8884 & 2.71 \\
\hline $\mathrm{H}-2$ & 74 & 28.4285 & 3.05 & 95 & 28.8773 & 2.72 \\
\hline $\mathrm{H}-2$ & 95 & 28.4335 & 3.05 & 122 & 28.7831 & 2.80 \\
\hline $\mathrm{H}-2$ & 116 & 28.3965 & 3.08 & 149 & 28.7311 & 2.84 \\
\hline $\mathrm{H}-2$ & 137 & 28.3219 & 3.16 & 176 & 28.7328 & 2.84 \\
\hline $\mathrm{H}-3$ & 9 & 27.6744 & 3.77 & 16 & 27.5907 & 3.83 \\
\hline $\mathrm{H}-3$ & 31 & 27.4352 & 3.99 & 42 & 27.5547 & 3.86 \\
\hline $\mathrm{H}-3$ & 52 & 27.4861 & 3.95 & 69 & 27.8377 & 3.61 \\
\hline $\mathrm{H}-3$ & 73 & 27.6908 & 3.75 & 96 & 27.7295 & 3.71 \\
\hline $\mathrm{H}-3$ & 94 & 27.4526 & 3.98 & 123 & 27.5883 & 3.83 \\
\hline $\mathrm{H}-3$ & 115 & 27.7304 & 3.71 & 150 & 27.7929 & 3.65 \\
\hline $\mathrm{H}-3$ & 136 & 27.4200 & 4.01 & 177 & 27.8530 & 3.60 \\
\hline $\mathrm{H}-4$ & 8 & 28.8044 & 2.70 & 17 & 28.5038 & 3.04 \\
\hline $\mathrm{H}-4$ & 30 & 28.5785 & 2.91 & 43 & 29.0932 & 2.53 \\
\hline $\mathrm{H}-4$ & 51 & 28.7199 & 2.78 & 70 & 28.2756 & 3.24 \\
\hline $\mathrm{H}-4$ & 72 & 28.7877 & 2.71 & 97 & 29.1889 & 2.45 \\
\hline
\end{tabular}




\begin{tabular}{|c|c|c|c|c|c|c|}
\hline $\mathrm{H}-4$ & 93 & 28.6106 & 2.88 & 124 & 28.7436 & 2.83 \\
\hline $\mathrm{H}-4$ & 114 & 28.7466 & 2.75 & 151 & 28.2311 & 3.28 \\
\hline $\mathrm{H}-4$ & 135 & 28.6072 & 2.89 & 178 & 28.9522 & 2.65 \\
\hline $\mathrm{H}-5$ & 7 & 27.2487 & 4.17 & 18 & 27.6154 & 3.81 \\
\hline $\mathrm{H}-5$ & 29 & 27.3210 & 4.10 & 44 & 27.3844 & 4.00 \\
\hline $\mathrm{H}-5$ & 50 & 27.3976 & 4.03 & 71 & 27.6777 & 3.75 \\
\hline $\mathrm{H}-5$ & 71 & 27.2157 & 4.20 & 98 & 27.4801 & 3.92 \\
\hline $\mathrm{H}-5$ & 92 & 27.4097 & 4.02 & 125 & 27.7414 & 3.70 \\
\hline $\mathrm{H}-5$ & 113 & 27.1789 & 4.24 & 152 & 27.8308 & 3.62 \\
\hline $\mathrm{H}-5$ & 134 & 27.3485 & 4.08 & 179 & 27.5541 & 3.86 \\
\hline H-6 & 17 & 27.4861 & 3.95 & 19 & 27.6899 & 3.74 \\
\hline H-6 & 19 & 28.0151 & 3.45 & 45 & 27.9517 & 3.52 \\
\hline H-6 & 38 & 27.4016 & 4.03 & 46 & 27.8292 & 3.62 \\
\hline H-6 & 40 & 27.8877 & 3.57 & 72 & 27.4497 & 3.95 \\
\hline H-6 & 59 & 26.9697 & 4.43 & 73 & 28.2577 & 3.25 \\
\hline H-6 & 61 & 28.5071 & 2.98 & 99 & 28.2374 & 3.27 \\
\hline H-6 & 80 & 27.3136 & 4.11 & 100 & 27.5055 & 3.90 \\
\hline H-6 & 82 & 28.0528 & 3.41 & 126 & 28.3169 & 3.20 \\
\hline H-6 & 101 & 27.5298 & 3.90 & 127 & 27.5424 & 3.87 \\
\hline H-6 & 103 & 27.9966 & 3.46 & 153 & 27.4665 & 3.93 \\
\hline H-6 & 122 & 27.5333 & 3.90 & 154 & 28.1069 & 3.38 \\
\hline H-6 & 124 & 28.0943 & 3.37 & 180 & 27.9108 & 3.55 \\
\hline H-6 & 142 & 27.2332 & 4.19 & 181 & 28.0632 & 3.42 \\
\hline H-6 & 144 & 28.1757 & 3.29 & 189 & 28.3100 & 3.21 \\
\hline $\mathrm{H}-7$ & 169 & 27.9701 & 3.49 & 20 & 28.0893 & 3.40 \\
\hline $\mathrm{H}-7$ & 170 & 27.9518 & 3.51 & 21 & 28.3444 & 3.18 \\
\hline H-7 & 171 & 28.3334 & 3.14 & 22 & 27.4798 & 3.92 \\
\hline $\mathrm{H}-7$ & 172 & 27.8792 & 3.57 & 47 & 28.1043 & 3.38 \\
\hline $\mathrm{H}-7$ & 173 & 28.1356 & 3.33 & 48 & 28.3973 & 3.13 \\
\hline $\mathrm{H}-7$ & 174 & 27.2323 & 4.19 & 49 & 27.4288 & 3.97 \\
\hline $\mathrm{H}-7$ & 175 & 27.8932 & 3.56 & 74 & 28.1006 & 3.39 \\
\hline $\mathrm{H}-7$ & 176 & 28.1336 & 3.33 & 75 & 28.2817 & 3.23 \\
\hline $\mathrm{H}-7$ & 177 & 27.2250 & 4.19 & 76 & 28.6132 & 2.95 \\
\hline $\mathrm{H}-7$ & 178 & 27.9306 & 3.53 & 101 & 28.0571 & 3.43 \\
\hline $\mathrm{H}-7$ & 179 & 28.0267 & 3.43 & 102 & 28.4072 & 3.12 \\
\hline $\mathrm{H}-7$ & 180 & 28.3324 & 3.15 & 103 & 27.3654 & 4.02 \\
\hline $\mathrm{H}-7$ & 181 & 27.9124 & 3.54 & 128 & 28.0646 & 3.42 \\
\hline $\mathrm{H}-7$ & 182 & 28.1367 & 3.33 & 129 & 28.3468 & 3.18 \\
\hline H-7 & 183 & 27.3214 & 4.10 & 130 & 27.4935 & 3.91 \\
\hline $\mathrm{H}-7$ & 184 & 27.9533 & 3.50 & 155 & 27.9868 & 3.49 \\
\hline $\mathrm{H}-7$ & 185 & 27.9811 & 3.48 & 156 & 28.2157 & 3.29 \\
\hline $\mathrm{H}-7$ & 186 & 28.3437 & 3.13 & 157 & 28.5527 & 3.00 \\
\hline $\mathrm{H}-7$ & 187 & 27.8609 & 3.59 & 182 & 28.0777 & 3.41 \\
\hline
\end{tabular}




\begin{tabular}{|c|c|c|c|c|c|c|}
\hline H-7 & 188 & 28.0969 & 3.37 & 183 & 28.0065 & 3.47 \\
\hline H-7 & 189 & 27.2010 & 4.22 & 184 & 28.5780 & 2.98 \\
\hline H-8 & 148 & 28.7861 & 2.72 & 23 & 28.2387 & 3.27 \\
\hline H-8 & 149 & 28.4630 & 3.02 & 24 & 28.6144 & 2.95 \\
\hline H-8 & 150 & 27.8671 & 3.59 & 25 & 27.9950 & 3.48 \\
\hline H-8 & 151 & 28.0947 & 3.37 & 50 & 28.3451 & 3.18 \\
\hline H-8 & 152 & 28.1653 & 3.30 & 51 & 28.3248 & 3.19 \\
\hline H-8 & 153 & 28.0808 & 3.38 & 52 & 28.9057 & 2.69 \\
\hline H-8 & 154 & 27.9711 & 3.49 & 77 & 28.2688 & 3.24 \\
\hline H-8 & 155 & 27.9675 & 3.49 & 78 & 28.5689 & 2.98 \\
\hline H-8 & 156 & 28.3967 & 3.08 & 79 & 27.9647 & 3.50 \\
\hline H-8 & 157 & 28.7363 & 2.76 & 104 & 28.5608 & 2.99 \\
\hline H-8 & 158 & 28.3016 & 3.17 & 105 & 28.4161 & 3.12 \\
\hline H-8 & 159 & 27.6400 & 3.80 & 106 & 28.1699 & 3.33 \\
\hline H-8 & 160 & 28.1468 & 3.32 & 131 & 27.9514 & 3.52 \\
\hline H-8 & 161 & 28.0057 & 3.45 & 132 & 28.3920 & 3.14 \\
\hline H-8 & 162 & 27.8926 & 3.56 & 133 & 28.4879 & 3.05 \\
\hline H-8 & 163 & 28.1957 & 3.27 & 158 & 28.2356 & 3.27 \\
\hline H-8 & 164 & 28.2565 & 3.22 & 159 & 28.6672 & 2.90 \\
\hline H-8 & 165 & 28.5114 & 2.98 & 160 & 28.0379 & 3.44 \\
\hline H-8 & 166 & 27.9270 & 3.53 & 185 & 28.3568 & 3.17 \\
\hline H-8 & 167 & 28.0646 & 3.40 & 186 & 28.1888 & 3.31 \\
\hline H-8 & 168 & 28.2456 & 3.23 & 187 & 28.9416 & 2.66 \\
\hline $\mathrm{HO}(-3)$ & 20 & 26.0685 & 5.29 & 26 & 26.8516 & 4.46 \\
\hline $\mathrm{HO}(-3)$ & 41 & 26.2463 & 5.12 & 53 & 26.6245 & 4.66 \\
\hline $\mathrm{HO}(-3)$ & 62 & 26.2547 & 5.11 & 80 & 26.9678 & 4.36 \\
\hline $\mathrm{HO}(-3)$ & 83 & 26.0041 & 5.35 & 107 & 26.6315 & 4.65 \\
\hline $\mathrm{HO}(-3)$ & 104 & 26.3292 & 5.04 & 134 & 26.5776 & 4.70 \\
\hline $\mathrm{HO}(-3)$ & 125 & 26.1043 & 5.25 & 161 & 27.0497 & 4.29 \\
\hline $\mathrm{HO}(-3)$ & 145 & 26.4584 & 4.92 & 188 & 26.8093 & 4.50 \\
\hline
\end{tabular}

\begin{tabular}{|c|c|c|c|c|c|c|}
\hline \multirow{2}{*}{$\begin{array}{l}\text { Atom } \\
\text { no. } \\
\text { in Fig. } \\
\text { S2b }\end{array}$} & \multicolumn{3}{|c|}{ W1 B3LYP } & \multicolumn{3}{|c|}{ BOYFOK03 M062X } \\
\hline & $\begin{array}{c}\text { Atom } \\
\text { no. in } \\
\text { Table S9 }\end{array}$ & Isotropic & $\delta_{\text {calc }}$ & $\begin{array}{c}\text { Atom } \\
\text { no. in } \\
\text { Table S10 } \\
\end{array}$ & Isotropic & $\delta_{\text {calc }}$ \\
\hline $\mathrm{C} 1$ & 6 & 92.0130 & 100.96 & 1 & 91.7892 & 96.45 \\
\hline $\mathrm{C} 1$ & 28 & 88.0065 & 105.10 & 27 & 92.2471 & 96.04 \\
\hline $\mathrm{C} 1$ & 49 & 88.8890 & 104.19 & 54 & 95.3537 & 93.22 \\
\hline $\mathrm{C} 1$ & 70 & 92.7995 & 100.15 & 81 & 92.2851 & 96.00 \\
\hline $\mathrm{C} 1$ & 91 & 88.7145 & 104.37 & 108 & 92.4030 & 95.89 \\
\hline $\mathrm{C} 1$ & 112 & 92.4572 & 100.50 & 135 & 95.2146 & 93.35 \\
\hline $\mathrm{C} 1$ & 133 & 88.3899 & 104.70 & 162 & 95.7332 & 92.88 \\
\hline $\mathrm{C} 2$ & 5 & 109.8332 & 82.56 & 2 & 112.1627 & 77.99 \\
\hline
\end{tabular}




\begin{tabular}{|c|c|c|c|c|c|c|}
\hline $\mathrm{C} 2$ & 27 & 109.3001 & 83.12 & 28 & 112.5141 & 77.67 \\
\hline $\mathrm{C} 2$ & 48 & 109.0451 & 83.38 & 55 & 110.0246 & 79.93 \\
\hline $\mathrm{C} 2$ & 69 & 108.9672 & 83.46 & 82 & 112.4567 & 77.72 \\
\hline $\mathrm{C} 2$ & 90 & 109.3849 & 83.03 & 109 & 113.0464 & 77.19 \\
\hline $\mathrm{C} 2$ & 111 & 108.3135 & 84.13 & 136 & 111.0327 & 79.01 \\
\hline $\mathrm{C} 2$ & 132 & 109.8661 & 82.53 & 163 & 111.5351 & 78.56 \\
\hline C3 & 4 & 117.6052 & 74.54 & 3 & 118.7960 & 71.98 \\
\hline $\mathrm{C} 3$ & 26 & 114.8693 & 77.37 & 29 & 118.8886 & 71.89 \\
\hline $\mathrm{C} 3$ & 47 & 114.0432 & 78.22 & 56 & 122.6384 & 68.50 \\
\hline $\mathrm{C} 3$ & 68 & 117.6814 & 74.46 & 83 & 117.7428 & 72.93 \\
\hline $\mathrm{C} 3$ & 89 & 115.3023 & 76.92 & 110 & 120.7421 & 70.22 \\
\hline $\mathrm{C} 3$ & 110 & 117.3606 & 74.79 & 137 & 121.9128 & 69.15 \\
\hline $\mathrm{C} 3$ & 131 & 114.5316 & 77.71 & 164 & 122.0207 & 69.06 \\
\hline $\mathrm{C} 4$ & 3 & 106.0299 & 86.49 & 4 & 111.5714 & 78.52 \\
\hline $\mathrm{C} 4$ & 25 & 107.7663 & 84.70 & 30 & 109.1734 & 80.70 \\
\hline $\mathrm{C} 4$ & 46 & 105.1837 & 87.36 & 57 & 111.3047 & 78.77 \\
\hline $\mathrm{C} 4$ & 67 & 105.9884 & 86.53 & 84 & 109.3543 & 80.53 \\
\hline $\mathrm{C} 4$ & 88 & 108.3398 & 84.11 & 111 & 109.6496 & 80.27 \\
\hline $\mathrm{C} 4$ & 109 & 105.5351 & 87.00 & 138 & 112.2882 & 77.88 \\
\hline $\mathrm{C} 4$ & 130 & 106.7117 & 85.79 & 165 & 109.1794 & 80.69 \\
\hline C5 & 2 & 123.5467 & 68.41 & 5 & 125.3299 & 66.06 \\
\hline C5 & 24 & 124.0695 & 67.87 & 31 & 129.5882 & 62.20 \\
\hline $\mathrm{C} 5$ & 45 & 120.3446 & 71.71 & 58 & 123.2576 & 67.94 \\
\hline $\mathrm{C} 5$ & 66 & 124.2874 & 67.64 & 85 & 129.6554 & 62.14 \\
\hline C5 & 87 & 122.9859 & 68.99 & 112 & 124.4275 & 66.88 \\
\hline C5 & 108 & 125.6813 & 66.20 & 139 & 122.2388 & 68.86 \\
\hline C5 & 129 & 119.4999 & 72.59 & 166 & 128.7686 & 62.94 \\
\hline C6 & 12 & 118.6915 & 73.42 & 6 & 125.8594 & 65.58 \\
\hline C6 & 34 & 119.0039 & 73.10 & 32 & 121.2703 & 69.74 \\
\hline C6 & 55 & 118.7226 & 73.39 & 59 & 123.1964 & 67.99 \\
\hline C6 & 76 & 119.2035 & 72.89 & 86 & 122.2980 & 68.81 \\
\hline C6 & 97 & 119.7987 & 72.28 & 113 & 122.0986 & 68.99 \\
\hline C6 & 118 & 119.8514 & 72.22 & 140 & 123.7253 & 67.51 \\
\hline C6 & 139 & 120.0168 & 72.05 & 167 & 120.2990 & 70.62 \\
\hline $\mathrm{C} 7$ & 21 & 134.3749 & 57.23 & 7 & 136.0686 & 56.33 \\
\hline $\mathrm{C} 7$ & 42 & 132.1479 & 59.53 & 33 & 136.2118 & 56.20 \\
\hline $\mathrm{C} 7$ & 63 & 132.0807 & 59.60 & 60 & 138.4825 & 54.14 \\
\hline $\mathrm{C} 7$ & 84 & 134.4403 & 57.16 & 87 & 136.7289 & 55.73 \\
\hline $\mathrm{C} 7$ & 105 & 131.7756 & 59.91 & 114 & 135.9982 & 56.39 \\
\hline $\mathrm{C} 7$ & 126 & 134.1818 & 57.43 & 141 & 139.0413 & 53.63 \\
\hline $\mathrm{C} 7$ & 146 & 132.3105 & 59.36 & 168 & 139.1860 & 53.50 \\
\hline $\mathrm{C} 8$ & 22 & 135.2010 & 56.38 & 8 & 138.3543 & 54.26 \\
\hline $\mathrm{C} 8$ & 43 & 134.4688 & 57.13 & 34 & 138.7343 & 53.91 \\
\hline
\end{tabular}




\begin{tabular}{lrrrrrr}
$\mathrm{C} 8$ & 64 & 134.5175 & 57.08 & 61 & 138.3371 & 54.27 \\
$\mathrm{C} 8$ & 85 & 135.8689 & 55.69 & 88 & 141.2081 & 51.67 \\
$\mathrm{C} 8$ & 106 & 132.7974 & 58.86 & 115 & 138.5428 & 54.09 \\
$\mathrm{C} 8$ & 127 & 135.0622 & 56.52 & 142 & 138.0922 & 54.49 \\
$\mathrm{C} 8$ & 147 & 133.9329 & 57.69 & 169 & 138.8591 & 53.80 \\
\hline
\end{tabular}



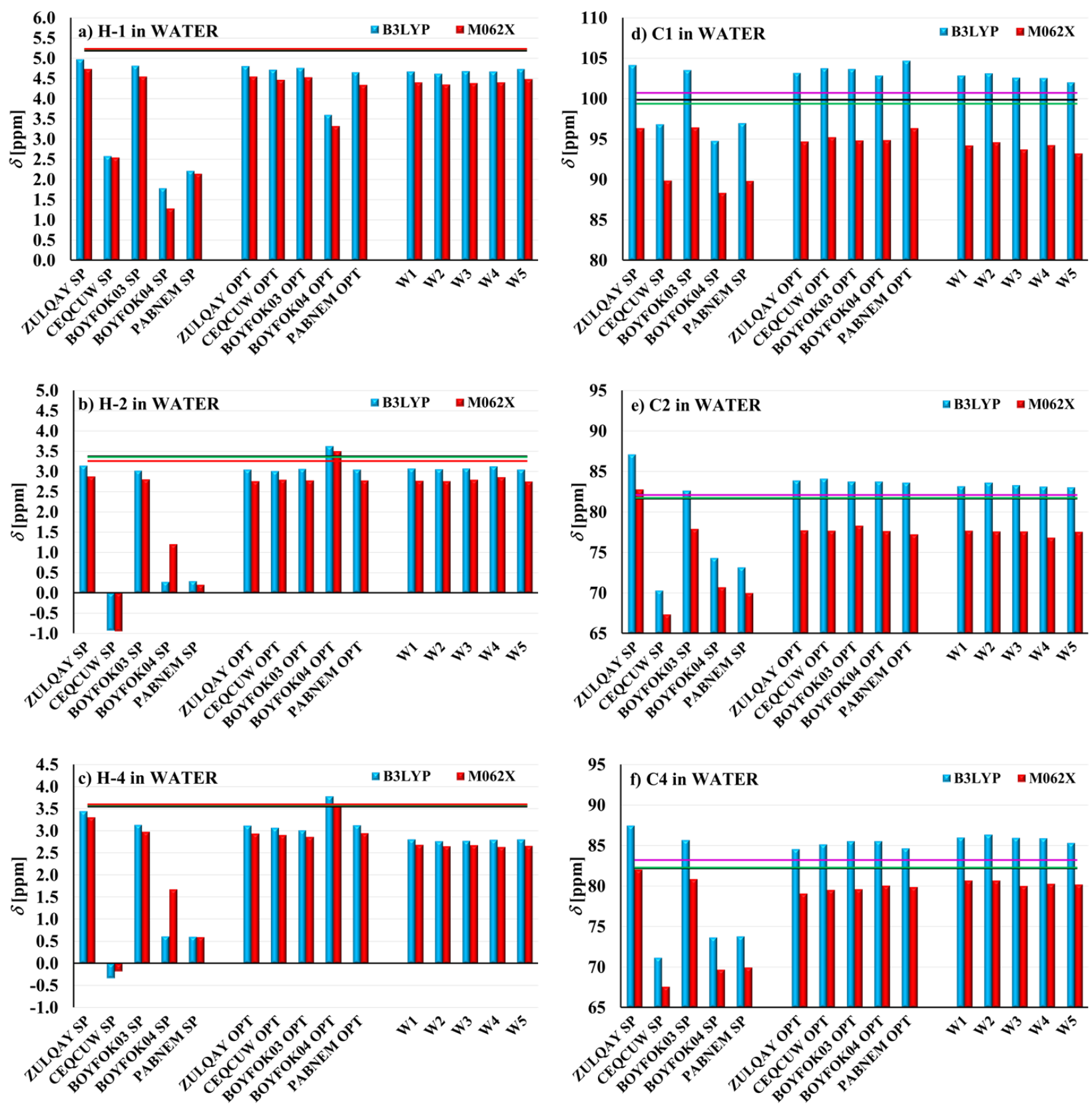

Figure S9. The ${ }^{1} \mathrm{H}$ (a-c) and ${ }^{13} \mathrm{C}$ NMR chemical shifts $\delta$ (scaled) for selected protons and carbons in various structures of DM- $\beta-\mathrm{CD}$ computed in water (PCM), compared to the experimental values measured in $\mathrm{D}_{2} \mathrm{O}$ (horizontal lines). ${ }^{11-14}$ The computed values were obtained from the calculations performed in water (PCM) at the two theory levels: B3LYP/6-31++G(d,p)//B3LYP-GD2/631G(d,p) (blue vertical bars) and M062X-GD3/6-31++G(d,p)//M062X-GD3/6-31G(d,p) (red vertical bars). 

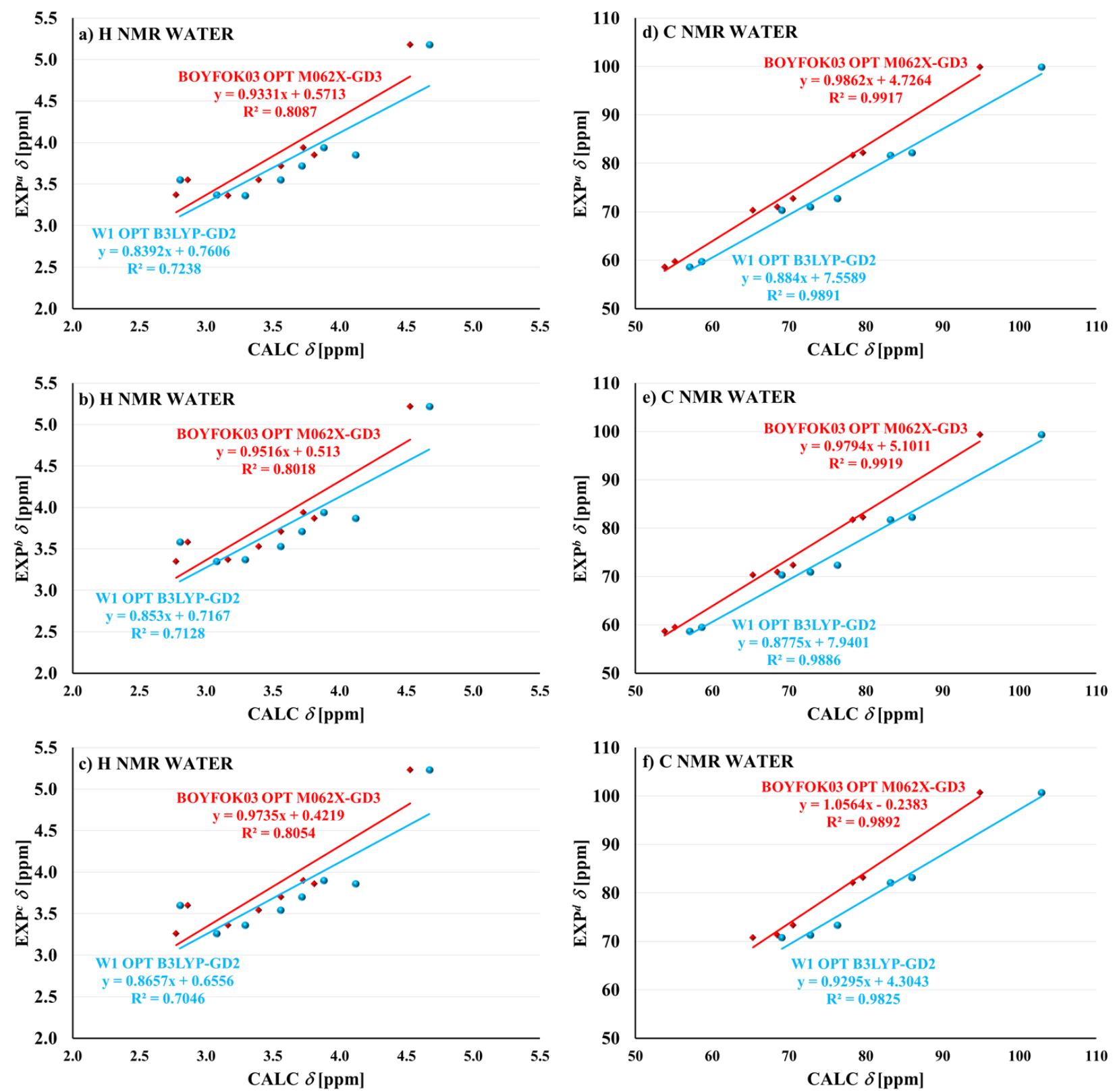

Figure S10. The linear regression between the ${ }^{1} \mathrm{H}$ and ${ }^{13} \mathrm{C}$ NMR chemical shifts $\delta$ measured in $\mathrm{D}_{2} \mathrm{O}$ and calculated in water (as averages over $\delta$ values for all equivalent atoms present in DM- $\beta-C D)$, corresponding to the results presented in Fig. 5 in the main article. The following notation is used: EXP - experimental data $\left({ }^{a}\right.$ ref. $11,{ }^{b}$ ref. $12,{ }^{c}$ ref. $13,{ }^{d}$ ref. 14$)$, CALC - calculated $\delta$ values (BOYFOK03 OPT M062X-GD3 - obtained from the M062X/6-31++G(d,p) calculations for the crystal structure BOYFOK03 optimized with the M062X-GD3/6-31G(d,p) method, W1 OPT B3LYP-GD2 - obtained from the B3LYP/6-31++G(d,p) calculations for the conformer W1 optimized with the B3LYP-GD2/6-31G(d,p) method). 
Table S6. The total electronic energies $(E)$, enthalpies $H$ and corrected Gibbs energies $\left(G_{\text {corr }}\right)$ obtained from the B3LYP-GD2/6-31G(d,p) and M062X-GD3/6-31G(d,p) calculations performed in water (PCM) for the optimized (OPT) structures of the molecule MIA and the 11 configurations of MIA:DM- $\beta$-CD shown and in Fig. 6 in the main article (B3LYP-GD2 based on the structure W1 of DM- $\beta-C D$ and M062X-GD3 based on the optimized BOYFOK03), as well as the corresponding BSSE corrected complexation energies, enthalpies and corrected Gibbs energies.

\begin{tabular}{|c|c|c|c|c|c|c|}
\hline \multicolumn{7}{|c|}{ B3LYP-GD2/6-31G(d,p) OPT } \\
\hline Structure & $\begin{array}{c}\boldsymbol{E} \\
\text { [hartree] }\end{array}$ & $\begin{array}{c}\boldsymbol{H} \\
\text { [hartree] }\end{array}$ & $\begin{array}{c}\boldsymbol{G}_{\text {corr }} \\
{[\text { hartree] }}\end{array}$ & $\begin{array}{c}\boldsymbol{E}_{\mathbf{c o m p l}}^{\mathbf{B S S E}} \\
{[\mathrm{kcal} / \mathrm{mol}]}\end{array}$ & $\begin{array}{c}\boldsymbol{H}_{\text {compl }}^{\text {BSSE }} \\
{[\mathrm{kcal} / \mathrm{mol}]}\end{array}$ & $\begin{array}{l}\boldsymbol{G}_{\text {corr_compl }}^{\text {BSSE }} \\
{[\mathrm{kcal} / \mathrm{mol}]}\end{array}$ \\
\hline MIA & -807.551356 & -807.191080 & -807.236438 & & & \\
\hline \multicolumn{7}{|c|}{ Complexes MIA:DM- $\beta-C D \_W 1:$} \\
\hline CR1 & -5633.673506 & -5631.600097 & -5631.823352 & -30.0 & -27.5 & -17.9 \\
\hline NR1 & -5633.670038 & -5631.595127 & -5631.817524 & -25.6 & -22.3 & -12.1 \\
\hline M1 & -5633.665950 & -5631.590865 & -5631.812970 & -25.7 & -22.2 & -11.9 \\
\hline F $\mathbf{\Lambda 1}$ & -5633.654559 & -5631.580928 & -5631.805435 & -20.2 & -17.6 & -8.8 \\
\hline FV1 & -5633.662649 & -5631.587641 & -5631.809570 & -23.3 & -19.9 & -9.4 \\
\hline CR2 & -5633.629793 & -5631.557523 & -5631.783449 & -11.3 & -9.6 & -1.6 \\
\hline NR2 & -5633.638187 & -5631.565903 & -5631.791783 & -16.7 & -14.9 & -7.0 \\
\hline M2 & -5633.628706 & -5631.556022 & -5631.781306 & -12.3 & -10.3 & -2.0 \\
\hline $\mathbf{F} \mathbf{\Lambda} 2$ & -5633.631248 & -5631.557737 & -5631.782403 & -12.6 & -10.0 & -1.3 \\
\hline FV2 & -5633.637132 & -5631.564299 & -5631.789100 & -15.5 & -13.4 & -4.8 \\
\hline $\mathbf{S}$ & -5633.630598 & -5631.558350 & -5631.785696 & -12.8 & -11.0 & -4.0 \\
\hline \multicolumn{7}{|c|}{ M062X-GD3/6-31G(d,p) OPT } \\
\hline MIA & -807.144935 & -806.780708 & -806.825847 & & & \\
\hline \multicolumn{7}{|c|}{ Complexes MIA:DM- $\beta$-CD_BOYFOK03: } \\
\hline CR1 & -5631.031856 & -5628.936882 & -5629.163499 & -14.4 & -12.6 & -2.7 \\
\hline NR1 & -5631.041248 & -5628.946418 & -5629.172434 & -20.9 & -19.2 & -9.0 \\
\hline M1 & -5631.032364 & -5628.938178 & -5629.165000 & -16.5 & -15.2 & -5.5 \\
\hline F $\Lambda 1$ & -5631.036597 & -5628.941696 & -5629.166441 & -18.3 & -16.5 & -5.5 \\
\hline FV1 & -5631.028068 & -5628.933587 & -5629.161460 & -13.9 & -12.4 & -3.3 \\
\hline CR2 & -5631.008651 & -5628.914577 & -5629.143098 & -6.9 & -5.7 & 3.0 \\
\hline NR2 & -5631.003218 & -5628.908404 & -5629.135399 & -3.0 & -1.3 & 8.3 \\
\hline M2 & -5631.015114 & -5628.921279 & -5629.150992 & -11.2 & -10.1 & -2.2 \\
\hline F $\Lambda 2$ & -5631.013055 & -5628.918482 & -5629.145901 & -9.3 & -7.7 & 1.6 \\
\hline FV2 & -5631.003217 & -5628.908416 & -5629.135429 & -3.0 & -1.4 & 8.3 \\
\hline $\mathbf{S}$ & -5631.009524 & -5628.915556 & -5629.145038 & -8.7 & -7.6 & 0.5 \\
\hline
\end{tabular}


Table S7. The total electronic energies $(E)$ obtained from the single point calculations B3LYP-GD2/6-31++G(d,p) and M062X-GD3/6-31++G(d,p) performed in water (PCM) for MIA and the eleven MIA:DM- $\beta$-CD structures shown in Fig. 6 in the main article, and the corresponding BSSE corrected complexation energies and Gibbs energies. The latter are calculated by adding to the SP energies the thermal corrections obtained from the values (OPT) given in Table S6.

\begin{tabular}{|c|c|c|c|}
\hline \multicolumn{4}{|c|}{ B3LYP-GD2/6-31++G(d,p) SP } \\
\hline Structure & $\begin{array}{c}\boldsymbol{E} \\
{[\text { hartree] }}\end{array}$ & $\begin{array}{c}\boldsymbol{E}_{\text {compl }}^{\text {BSSE }} \\
{[\mathrm{kcal} / \mathrm{mol}]}\end{array}$ & $\begin{array}{l}\boldsymbol{G}_{\text {corr_compl }}^{\text {BSSE }} \\
{[\mathrm{kcal} / \mathrm{mol}]}\end{array}$ \\
\hline MIA & -807.577421 & & \\
\hline \multicolumn{4}{|c|}{ Complexes MIA:DM- $\beta-C D \_W 1:$} \\
\hline CR1 & -5633.855971 & -29.6 & -17.5 \\
\hline NR1 & -5633.848597 & -24.3 & -10.8 \\
\hline M1 & -5633.846785 & -22.8 & -9.0 \\
\hline FA1 & -5633.837500 & -18.3 & -6.8 \\
\hline FV1 & -5633.843966 & -22.1 & -8.2 \\
\hline CR2 & -5633.822921 & -10.8 & -1.1 \\
\hline NR2 & -5633.830932 & -16.1 & -6.4 \\
\hline M2 & -5633.823081 & -11.7 & -1.4 \\
\hline F $\mathbf{\Lambda} 2$ & -5633.824386 & -12.4 & -1.1 \\
\hline FV2 & -5633.830364 & -15.3 & -4.6 \\
\hline $\mathbf{S}$ & -5633.824781 & -12.6 & -3.8 \\
\hline \multicolumn{4}{|c|}{ M062X-GD3/6-31++G(d,p) SP } \\
\hline MIA & -807.166090 & & \\
\hline \multicolumn{4}{|c|}{ Complexes MIA:DM- $\beta$-CD_BOYFOKO3: } \\
\hline CR1 & -5631.189154 & -17.1 & -5.4 \\
\hline NR1 & -5631.201390 & -23.9 & -11.9 \\
\hline M1 & -5631.196101 & -19.6 & -8.6 \\
\hline $\mathbf{F} \mathbf{\Lambda} \mathbf{1}$ & -5631.196163 & -21.1 & -8.3 \\
\hline FV1 & -5631.190543 & -16.6 & -6.1 \\
\hline CR2 & -5631.173514 & -8.3 & 1.6 \\
\hline NR2 & -5631.166481 & -3.2 & 8.1 \\
\hline M2 & -5631.179517 & -13.0 & -4.0 \\
\hline FA2 & -5631.176848 & -10.2 & 0.8 \\
\hline FV2 & -5631.166492 & -3.2 & 8.1 \\
\hline $\mathbf{S}$ & -5631.175167 & -9.6 & -0.3 \\
\hline
\end{tabular}


Table S8. The computed ${ }^{1} \mathrm{H}$ and ${ }^{13} \mathrm{C}$ NMR chemical shifts $\delta$ [ppm] for individual atoms of DM- $\beta$ $\mathrm{CD}$ in the most stable conformers of MIA:DM- $\beta-\mathrm{CD}$ in water indicated by the methods B3LYPGD2 (CR1) and M062X-GD3 (NR1), obtained from the B3LYP/6-31++G(d,p)//B3LYP-GD2/6$31 \mathrm{G}(\mathrm{d}, \mathrm{p})$ and M062X/6-31++G(d,p)//M062X-GD3/6-31G(d,p) calculations, respectively. The $\delta$ values are scaled as described in Table S5.

\begin{tabular}{|c|c|c|c|c|c|c|}
\hline \multirow[b]{3}{*}{$\begin{array}{l}\text { Atom no. } \\
\text { in Fig. } \\
\text { S2b }\end{array}$} & \multicolumn{6}{|c|}{ a) Protons } \\
\hline & \multicolumn{3}{|c|}{ CR1 B3LYP } & \multicolumn{3}{|c|}{ NR1 M062X } \\
\hline & $\begin{array}{c}\text { Atom } \\
\text { no. in } \\
\text { Table S9 }\end{array}$ & Isotropic & $\delta$ & $\begin{array}{c}\text { Atom } \\
\text { no. in } \\
\text { Table } S 10\end{array}$ & Isotropic & $\delta$ \\
\hline $\mathrm{H}-1$ & 11 & 27.0276 & 4.38 & 14 & 26.9186 & 4.41 \\
\hline $\mathrm{H}-1$ & 33 & 26.9005 & 4.50 & 40 & 26.6941 & 4.60 \\
\hline $\mathrm{H}-1$ & 54 & 26.7429 & 4.65 & 67 & 26.9901 & 4.34 \\
\hline $\mathrm{H}-1$ & 75 & 27.2540 & 4.17 & 94 & 26.6348 & 4.65 \\
\hline $\mathrm{H}-1$ & 96 & 27.2060 & 4.21 & 121 & 27.0449 & 4.30 \\
\hline $\mathrm{H}-1$ & 117 & 26.6595 & 4.73 & 148 & 27.2445 & 4.13 \\
\hline $\mathrm{H}-1$ & 138 & 26.6655 & 4.72 & 175 & 26.8159 & 4.49 \\
\hline $\mathrm{H}-2$ & 10 & 28.5685 & 2.92 & 15 & 28.6282 & 2.93 \\
\hline $\mathrm{H}-2$ & 32 & 28.4221 & 3.06 & 41 & 29.0444 & 2.58 \\
\hline $\mathrm{H}-2$ & 53 & 28.0742 & 3.39 & 68 & 28.6909 & 2.88 \\
\hline $\mathrm{H}-2$ & 74 & 28.5064 & 2.98 & 95 & 28.7349 & 2.84 \\
\hline $\mathrm{H}-2$ & 95 & 28.7322 & 2.77 & 122 & 29.3048 & 2.35 \\
\hline $\mathrm{H}-2$ & 116 & 28.1601 & 3.31 & 149 & 29.0824 & 2.54 \\
\hline $\mathrm{H}-2$ & 137 & 28.4995 & 2.99 & 176 & 28.4153 & 3.12 \\
\hline $\mathrm{H}-3$ & 9 & 27.7715 & 3.68 & 16 & 28.0958 & 3.39 \\
\hline $\mathrm{H}-3$ & 31 & 27.5781 & 3.86 & 42 & 28.4067 & 3.12 \\
\hline $\mathrm{H}-3$ & 52 & 26.6528 & 4.73 & 69 & 26.8241 & 4.49 \\
\hline $\mathrm{H}-3$ & 73 & 27.4220 & 4.01 & 96 & 27.0934 & 4.26 \\
\hline $\mathrm{H}-3$ & 94 & 28.9871 & 2.53 & 123 & 30.3230 & 1.47 \\
\hline $\mathrm{H}-3$ & 115 & 26.9598 & 4.44 & 150 & 26.3543 & 4.89 \\
\hline $\mathrm{H}-3$ & 136 & 27.0282 & 4.38 & 177 & 26.5581 & 4.72 \\
\hline $\mathrm{H}-4$ & 8 & 28.9378 & 2.57 & 17 & 28.5581 & 2.99 \\
\hline $\mathrm{H}-4$ & 30 & 28.7988 & 2.70 & 43 & 29.3814 & 2.28 \\
\hline $\mathrm{H}-4$ & 51 & 28.7213 & 2.78 & 70 & 28.1721 & 3.33 \\
\hline $\mathrm{H}-4$ & 72 & 28.7163 & 2.78 & 97 & 29.3425 & 2.32 \\
\hline $\mathrm{H}-4$ & 93 & 29.4598 & 2.08 & 124 & 29.0276 & 2.59 \\
\hline $\mathrm{H}-4$ & 114 & 28.7037 & 2.79 & 151 & 28.1956 & 3.31 \\
\hline $\mathrm{H}-4$ & 135 & 28.5252 & 2.96 & 178 & 28.7458 & 2.83 \\
\hline $\mathrm{H}-5$ & 7 & 27.6707 & 3.77 & 18 & 28.4039 & 3.13 \\
\hline $\mathrm{H}-5$ & 29 & 27.3239 & 4.10 & 44 & 28.7868 & 2.80 \\
\hline $\mathrm{H}-5$ & 50 & 27.1825 & 4.23 & 71 & 27.6423 & 3.78 \\
\hline
\end{tabular}




\begin{tabular}{|c|c|c|c|c|c|c|}
\hline $\mathrm{H}-5$ & 71 & 27.6461 & 3.79 & 98 & 27.0135 & 4.32 \\
\hline $\mathrm{H}-5$ & 92 & 28.3206 & 3.16 & 125 & 27.7383 & 3.70 \\
\hline H-5 & 113 & 27.1245 & 4.29 & 152 & 27.0351 & 4.31 \\
\hline $\mathrm{H}-5$ & 134 & 27.0306 & 4.38 & 179 & 27.4846 & 3.92 \\
\hline H-6 & 17 & 27.3629 & 4.06 & 19 & 27.5150 & 3.89 \\
\hline H-6 & 19 & 28.0355 & 3.43 & 45 & 28.3989 & 3.13 \\
\hline H-6 & 38 & 27.4696 & 3.96 & 46 & 28.7909 & 2.79 \\
\hline H-6 & 40 & 27.9044 & 3.55 & 72 & 27.6981 & 3.73 \\
\hline H-6 & 59 & 26.7549 & 4.64 & 73 & 28.2134 & 3.29 \\
\hline H-6 & 61 & 28.4368 & 3.05 & 99 & 28.1934 & 3.31 \\
\hline H-6 & 80 & 27.2044 & 4.21 & 100 & 27.7096 & 3.72 \\
\hline H-6 & 82 & 28.2746 & 3.20 & 126 & 28.7371 & 2.84 \\
\hline H-6 & 101 & 27.5633 & 3.87 & 127 & 27.7513 & 3.69 \\
\hline H-6 & 103 & 28.4122 & 3.07 & 153 & 27.3179 & 4.06 \\
\hline H-6 & 122 & 27.6969 & 3.75 & 154 & 28.1706 & 3.33 \\
\hline H-6 & 124 & 27.9034 & 3.55 & 180 & 28.0134 & 3.46 \\
\hline H-6 & 142 & 26.7645 & 4.63 & 181 & 28.2606 & 3.25 \\
\hline H-6 & 144 & 28.4519 & 3.03 & 189 & 28.5050 & 3.04 \\
\hline H-7 & 169 & 27.9796 & 3.48 & 20 & 27.9107 & 3.55 \\
\hline $\mathrm{H}-7$ & 170 & 28.2898 & 3.19 & 21 & 28.2091 & 3.29 \\
\hline H-7 & 171 & 27.2582 & 4.16 & 22 & 27.1523 & 4.20 \\
\hline H-7 & 172 & 28.0273 & 3.43 & 47 & 28.0459 & 3.44 \\
\hline $\mathrm{H}-7$ & 173 & 28.1961 & 3.27 & 48 & 28.4652 & 3.07 \\
\hline $\mathrm{H}-7$ & 174 & 27.3224 & 4.10 & 49 & 27.2737 & 4.10 \\
\hline H-7 & 175 & 27.7970 & 3.65 & 74 & 27.7307 & 3.71 \\
\hline H-7 & 176 & 28.1107 & 3.35 & 75 & 28.1118 & 3.38 \\
\hline $\mathrm{H}-7$ & 177 & 26.7674 & 4.63 & 76 & 27.0704 & 4.28 \\
\hline $\mathrm{H}-7$ & 178 & 27.7090 & 3.74 & 101 & 27.9526 & 3.52 \\
\hline H-7 & 179 & 28.1361 & 3.33 & 102 & 28.3472 & 3.18 \\
\hline H-7 & 180 & 26.9199 & 4.48 & 103 & 27.2778 & 4.10 \\
\hline H-7 & 181 & 27.8646 & 3.59 & 128 & 28.0813 & 3.40 \\
\hline H-7 & 182 & 28.2346 & 3.24 & 129 & 28.4114 & 3.12 \\
\hline H-7 & 183 & 27.0329 & 4.37 & 130 & 27.3710 & 4.02 \\
\hline H-7 & 184 & 27.6997 & 3.74 & 155 & 28.1006 & 3.39 \\
\hline $\mathrm{H}-7$ & 185 & 27.9650 & 3.49 & 156 & 28.3870 & 3.14 \\
\hline H-7 & 186 & 26.9934 & 4.41 & 157 & 27.5192 & 3.89 \\
\hline H-7 & 187 & 27.8862 & 3.57 & 182 & 27.9330 & 3.53 \\
\hline H-7 & 188 & 28.1466 & 3.32 & 183 & 28.3524 & 3.17 \\
\hline H-7 & 189 & 27.1300 & 4.28 & 184 & 26.7219 & 4.58 \\
\hline H-8 & 148 & 28.3337 & 3.14 & 23 & 28.2609 & 3.25 \\
\hline H-8 & 149 & 27.6812 & 3.76 & 24 & 28.0750 & 3.41 \\
\hline H-8 & 150 & 27.4053 & 4.02 & 25 & 28.7660 & 2.81 \\
\hline $\mathrm{H}-8$ & 151 & 27.9037 & 3.55 & 50 & 28.8425 & 2.75 \\
\hline
\end{tabular}




\begin{tabular}{|c|c|c|c|c|c|c|}
\hline $\mathrm{H}-8$ & 152 & 27.7997 & 3.65 & 51 & 29.3977 & 2.27 \\
\hline H-8 & 153 & 28.0869 & 3.38 & 52 & 29.2672 & 2.38 \\
\hline H-8 & 154 & 28.0477 & 3.41 & 77 & 28.1326 & 3.36 \\
\hline H-8 & 155 & 27.8871 & 3.57 & 78 & 28.5060 & 3.04 \\
\hline H-8 & 156 & 28.4753 & 3.01 & 79 & 27.7096 & 3.72 \\
\hline H-8 & 157 & 27.9963 & 3.46 & 104 & 28.0308 & 3.45 \\
\hline $\mathrm{H}-8$ & 158 & 27.7388 & 3.71 & 105 & 27.9210 & 3.54 \\
\hline H-8 & 159 & 28.2579 & 3.22 & 106 & 27.9283 & 3.54 \\
\hline $\mathrm{H}-8$ & 160 & 28.6613 & 2.83 & 131 & 28.3048 & 3.21 \\
\hline H-8 & 161 & 28.2674 & 3.21 & 132 & 28.4922 & 3.05 \\
\hline $\mathrm{H}-8$ & 162 & 28.0134 & 3.45 & 133 & 27.8499 & 3.60 \\
\hline H-8 & 163 & 27.8726 & 3.58 & 158 & 28.2188 & 3.29 \\
\hline H-8 & 164 & 27.7665 & 3.68 & 159 & 28.5898 & 2.97 \\
\hline H-8 & 165 & 28.1539 & 3.31 & 160 & 28.0871 & 3.40 \\
\hline $\mathrm{H}-8$ & 166 & 27.8730 & 3.58 & 185 & 28.2859 & 3.23 \\
\hline H-8 & 167 & 27.9066 & 3.55 & 186 & 28.2294 & 3.28 \\
\hline H-8 & 168 & 28.3203 & 3.16 & 187 & 29.2278 & 2.42 \\
\hline $\mathrm{HO}(-3)$ & 20 & 25.7665 & 5.57 & 26 & 26.6266 & 4.66 \\
\hline $\mathrm{HO}(-3)$ & 41 & 26.1781 & 5.18 & 53 & 26.4247 & 4.83 \\
\hline $\mathrm{HO}(-3)$ & 62 & 26.7640 & 4.63 & 80 & 25.9674 & 5.23 \\
\hline $\mathrm{HO}(-3)$ & 83 & 25.4788 & 5.85 & 107 & 26.3334 & 4.91 \\
\hline $\mathrm{HO}(-3)$ & 104 & 27.1098 & 4.30 & 134 & 26.9944 & 4.34 \\
\hline $\mathrm{HO}(-3)$ & 125 & 25.8125 & 5.53 & 161 & 26.8233 & 4.49 \\
\hline $\mathrm{HO}(-3)$ & 145 & 25.7694 & 5.57 & 188 & 26.2787 & 4.96 \\
\hline \multicolumn{7}{|c|}{ b) Carbons } \\
\hline \multirow{2}{*}{$\begin{array}{l}\text { Atom } \\
\text { no. } \\
\text { in Fig. } \\
\text { S2b }\end{array}$} & \multicolumn{3}{|c|}{ CR1 B3L YP } & \multicolumn{3}{|c|}{ NR1 M062X } \\
\hline & $\begin{array}{c}\text { Atom } \\
\text { no. in } \\
\text { Table S9 }\end{array}$ & Isotropic & $\delta$ & $\begin{array}{c}\text { Atom } \\
\text { no. in } \\
\text { Table S10 }\end{array}$ & Isotropic & $\delta$ \\
\hline $\mathrm{C} 1$ & 6 & 88.8366 & 104.24 & 1 & 92.4731 & 95.83 \\
\hline $\mathrm{C} 1$ & 28 & 88.7002 & 104.38 & 27 & 94.9613 & 93.58 \\
\hline $\mathrm{C} 1$ & 49 & 86.6357 & 106.51 & 54 & 93.0368 & 95.32 \\
\hline $\mathrm{C} 1$ & 70 & 88.1708 & 104.93 & 81 & 91.1027 & 97.07 \\
\hline $\mathrm{C} 1$ & 91 & 89.7599 & 103.29 & 108 & 94.3126 & 94.16 \\
\hline $\mathrm{C} 1$ & 112 & 87.9803 & 105.12 & 135 & 95.0608 & 93.49 \\
\hline $\mathrm{C} 1$ & 133 & 89.1953 & 103.87 & 162 & 92.0381 & 96.22 \\
\hline $\mathrm{C} 2$ & 5 & 109.9182 & 82.48 & 2 & 112.8043 & 77.41 \\
\hline $\mathrm{C} 2$ & 27 & 108.9119 & 83.52 & 28 & 113.3558 & 76.91 \\
\hline $\mathrm{C} 2$ & 48 & 110.4756 & 81.90 & 55 & 112.2203 & 77.94 \\
\hline $\mathrm{C} 2$ & 69 & 107.9648 & 84.49 & 82 & 112.3675 & 77.80 \\
\hline $\mathrm{C} 2$ & 90 & 110.3486 & 82.03 & 109 & 113.2771 & 76.98 \\
\hline $\mathrm{C} 2$ & 111 & 109.0600 & 83.36 & 136 & 111.1915 & 78.87 \\
\hline $\mathrm{C} 2$ & 132 & 109.4669 & 82.94 & 163 & 113.7756 & 76.53 \\
\hline
\end{tabular}




\begin{tabular}{|c|c|c|c|c|c|c|}
\hline $\mathrm{C} 3$ & 4 & 114.8304 & 77.41 & 3 & 120.1989 & 70.71 \\
\hline $\mathrm{C} 3$ & 26 & 114.3359 & 77.92 & 29 & 120.6781 & 70.27 \\
\hline $\mathrm{C} 3$ & 47 & 116.7627 & 75.41 & 56 & 120.4031 & 70.52 \\
\hline $\mathrm{C} 3$ & 68 & 116.7953 & 75.38 & 83 & 117.2926 & 73.34 \\
\hline $\mathrm{C} 3$ & 89 & 117.6730 & 74.47 & 110 & 121.4185 & 69.60 \\
\hline C3 & 110 & 114.8464 & 77.39 & 137 & 123.1553 & 68.03 \\
\hline $\mathrm{C} 3$ & 131 & 116.2707 & 75.92 & 164 & 118.9935 & 71.80 \\
\hline $\mathrm{C} 4$ & 3 & 106.3629 & 86.15 & 4 & 116.3174 & 74.22 \\
\hline $\mathrm{C} 4$ & 25 & 107.8127 & 84.65 & 30 & 110.9498 & 79.09 \\
\hline $\mathrm{C} 4$ & 46 & 104.3072 & 88.27 & 57 & 112.6575 & 77.54 \\
\hline $\mathrm{C} 4$ & 67 & 103.2829 & 89.33 & 84 & 109.3323 & 80.55 \\
\hline $\mathrm{C} 4$ & 88 & 106.1817 & 86.33 & 111 & 118.8941 & 71.89 \\
\hline $\mathrm{C} 4$ & 109 & 108.5776 & 83.86 & 138 & 111.2427 & 78.82 \\
\hline $\mathrm{C} 4$ & 130 & 104.5718 & 88.00 & 165 & 109.7747 & 80.15 \\
\hline $\mathrm{C} 5$ & 2 & 122.9117 & 69.06 & 5 & 122.5520 & 68.58 \\
\hline $\mathrm{C} 5$ & 24 & 125.0786 & 66.83 & 31 & 128.2935 & 63.37 \\
\hline $\mathrm{C} 5$ & 45 & 118.7078 & 73.40 & 58 & 122.3590 & 68.75 \\
\hline $\mathrm{C} 5$ & 66 & 121.1121 & 70.92 & 85 & 126.9301 & 64.61 \\
\hline $\mathrm{C} 5$ & 87 & 125.2587 & 66.64 & 112 & 118.7864 & 71.99 \\
\hline C5 & 108 & 124.3657 & 67.56 & 139 & 121.5626 & 69.47 \\
\hline $\mathrm{C} 5$ & 129 & 119.6186 & 72.46 & 166 & 129.7001 & 62.10 \\
\hline C6 & 12 & 117.0810 & 75.08 & 6 & 125.7135 & 65.71 \\
\hline C6 & 34 & 119.5777 & 72.51 & 32 & 121.7886 & 69.27 \\
\hline C6 & 55 & 118.9787 & 73.12 & 59 & 122.7450 & 68.40 \\
\hline C6 & 76 & 119.7320 & 72.35 & 86 & 119.8168 & 71.05 \\
\hline C6 & 97 & 120.0710 & 72.00 & 113 & 125.4632 & 65.94 \\
\hline C6 & 118 & 119.4568 & 72.63 & 140 & 124.6450 & 66.68 \\
\hline C6 & 139 & 119.0251 & 73.08 & 167 & 122.5024 & 68.62 \\
\hline $\mathrm{C} 7$ & 21 & 132.3585 & 59.31 & 7 & 136.2223 & 56.19 \\
\hline $\mathrm{C} 7$ & 42 & 133.1191 & 58.53 & 33 & 137.0557 & 55.43 \\
\hline $\mathrm{C} 7$ & 63 & 132.5287 & 59.14 & 60 & 136.5127 & 55.93 \\
\hline $\mathrm{C} 7$ & 84 & 132.5530 & 59.11 & 87 & 136.1993 & 56.21 \\
\hline $\mathrm{C} 7$ & 105 & 132.4335 & 59.23 & 114 & 135.3672 & 56.96 \\
\hline $\mathrm{C} 7$ & 126 & 131.9203 & 59.76 & 141 & 136.0776 & 56.32 \\
\hline $\mathrm{C} 7$ & 146 & 131.8570 & 59.83 & 168 & 136.5944 & 55.85 \\
\hline $\mathrm{C} 8$ & 22 & 133.2857 & 58.35 & 8 & 136.5136 & 55.92 \\
\hline $\mathrm{C} 8$ & 43 & 134.5275 & 57.07 & 34 & 136.7405 & 55.72 \\
\hline $\mathrm{C} 8$ & 64 & 136.1837 & 55.36 & 61 & 137.7881 & 54.77 \\
\hline $\mathrm{C} 8$ & 85 & 132.9076 & 58.75 & 88 & 137.8103 & 54.75 \\
\hline $\mathrm{C} 8$ & 106 & 132.3005 & 59.37 & 115 & 135.9630 & 56.42 \\
\hline $\mathrm{C} 8$ & 127 & 133.0478 & 58.60 & 142 & 138.8556 & 53.80 \\
\hline $\mathrm{C} 8$ & 147 & 134.4538 & 57.15 & 169 & 140.2172 & 52.57 \\
\hline
\end{tabular}


Table S9. Cartesian coordinates of atoms in the lowest energy structures of DM- $\beta-C D$ in vacuo (V1) and in water (W1) obtained from the B3LYP-GD2/6-31G(d,p) calculations. The true minima are confirmed by zero imaginary frequencies.

\begin{tabular}{|c|c|c|c|c|c|c|c|}
\hline & & & V1 & & & W1 & \\
\hline No. & Atom & $\mathbf{x}$ & $\mathbf{y}$ & $\mathbf{z}$ & $\mathbf{x}$ & $\mathbf{y}$ & $\mathbf{z}$ \\
\hline 1 & $\mathrm{O}$ & -3.0703 & -5.4790 & 0.5019 & 3.5658 & -4.2864 & 1.4784 \\
\hline 2 & $\mathrm{C}$ & -3.5932 & -4.1510 & 0.6921 & 2.1580 & -4.1601 & 1.1930 \\
\hline 3 & $\mathrm{C}$ & -4.2955 & -3.6969 & -0.5919 & 1.7720 & -5.1899 & 0.1293 \\
\hline 4 & $\mathrm{C}$ & -3.4247 & -3.8465 & -1.8478 & 2.6458 & -5.0929 & -1.1265 \\
\hline 5 & $\mathrm{C}$ & -2.8111 & -5.2434 & -1.8979 & 4.1186 & -5.1154 & -0.7419 \\
\hline 6 & $\mathrm{C}$ & -2.1529 & -5.5784 & -0.5549 & 4.3977 & -4.0728 & 0.3625 \\
\hline 7 & $\mathrm{H}$ & 1.5722 & -1.9028 & 2.1638 & 1.9534 & -3.1560 & 0.8042 \\
\hline 8 & $\mathrm{H}$ & -5.2062 & -4.2951 & -0.7414 & 1.8748 & -6.1987 & 0.5511 \\
\hline 9 & $\mathrm{H}$ & -2.6006 & -3.1219 & -1.7708 & 2.4326 & -4.1236 & -1.6019 \\
\hline 10 & $\mathrm{H}$ & -3.6128 & -5.9753 & -2.0756 & 4.3702 & -6.1104 & -0.3480 \\
\hline 11 & $\mathrm{H}$ & -1.8054 & -6.6160 & -0.5643 & 5.4207 & -4.1725 & 0.7376 \\
\hline 12 & $\mathrm{C}$ & -4.5514 & -4.2373 & 1.8741 & 1.4131 & -4.3840 & 2.5032 \\
\hline 13 & $\mathrm{O}$ & -4.6216 & -2.3088 & -0.5231 & 0.4249 & -4.9541 & -0.2874 \\
\hline 14 & $\mathrm{O}$ & -4.2000 & -3.6380 & -3.0120 & 2.3741 & -6.1696 & -2.0012 \\
\hline 15 & $\mathrm{O}$ & -1.8004 & -5.3775 & -2.8944 & 4.8925 & -4.8449 & -1.9056 \\
\hline 16 & $\mathrm{O}$ & -1.0659 & -4.6952 & -0.3789 & 4.1920 & -2.7877 & -0.1839 \\
\hline 17 & $\mathrm{H}$ & -5.0943 & -3.2862 & 1.9685 & 0.3743 & -4.6683 & 2.2934 \\
\hline 18 & $\mathrm{O}$ & -3.8877 & -4.5711 & 3.0784 & 1.4579 & -3.2526 & 3.3648 \\
\hline 19 & $\mathrm{H}$ & -5.2688 & -5.0420 & 1.6679 & 1.8998 & -5.2093 & 3.0351 \\
\hline 20 & $\mathrm{H}$ & -4.7504 & -2.8431 & -2.8691 & 1.4282 & -6.1067 & -2.2481 \\
\hline 21 & $\mathrm{C}$ & -2.2872 & -5.6438 & -4.2100 & 6.2679 & -5.2104 & -1.7778 \\
\hline 22 & $\mathrm{C}$ & -3.4177 & -3.4239 & 3.7762 & 0.5052 & -2.2602 & 2.9935 \\
\hline 23 & $\mathrm{O}$ & 1.9446 & -4.8315 & 1.6677 & 6.1395 & 0.1790 & 0.7607 \\
\hline 24 & $\mathrm{C}$ & 0.7118 & -4.2652 & 1.1672 & 5.0913 & -0.7985 & 0.8979 \\
\hline 25 & $\mathrm{C}$ & 0.1138 & -5.2579 & 0.1756 & 5.2787 & -1.8617 & -0.1861 \\
\hline 26 & $\mathrm{C}$ & 1.0839 & -5.5424 & -0.9783 & 5.2808 & -1.2309 & -1.5835 \\
\hline 27 & $\mathrm{C}$ & 2.4218 & -6.0139 & -0.4145 & 6.2719 & -0.0692 & -1.6495 \\
\hline 28 & $\mathrm{C}$ & 2.9146 & -5.0361 & 0.6688 & 6.0768 & 0.8846 & -0.4598 \\
\hline 29 & $\mathrm{H}$ & 5.9175 & -0.8662 & 3.6227 & 4.1187 & -0.3183 & 0.7421 \\
\hline 30 & $\mathrm{H}$ & -0.1123 & -6.1953 & 0.7031 & 6.2332 & -2.3832 & -0.0314 \\
\hline 31 & $\mathrm{H}$ & 1.2545 & -4.5946 & -1.5089 & 4.2743 & -0.8218 & -1.7593 \\
\hline 32 & $\mathrm{H}$ & 2.2812 & -7.0015 & 0.0501 & 7.2925 & -0.4720 & -1.5921 \\
\hline 33 & $\mathrm{H}$ & 3.7892 & -5.4571 & 1.1765 & 6.8929 & 1.6130 & -0.4317 \\
\hline 34 & $\mathrm{C}$ & -0.1968 & -3.9519 & 2.3645 & 5.1668 & -1.3435 & 2.3151 \\
\hline 35 & $\mathrm{O}$ & 0.5500 & -6.5269 & -1.8442 & 5.6241 & -2.1910 & -2.5671 \\
\hline 36 & $\mathrm{O}$ & 3.4613 & -6.0791 & -1.3816 & 6.1178 & 0.7075 & -2.8315 \\
\hline 37 & $\mathrm{O}$ & 3.2446 & -3.8184 & 0.0394 & 4.8345 & 1.5276 & -0.6149 \\
\hline 38 & $\mathrm{H}$ & -1.1959 & -4.3669 & 2.2060 & 4.5673 & -2.2614 & 2.3813 \\
\hline 39 & $\mathrm{O}$ & -0.3792 & -2.5570 & 2.5619 & 4.7274 & -0.3910 & 3.2771 \\
\hline 40 & $\mathrm{H}$ & 0.2402 & -4.4174 & 3.2623 & 6.2107 & -1.5782 & 2.5505 \\
\hline 41 & $\mathrm{H}$ & -0.2210 & -6.1233 & -2.2941 & 5.1013 & -2.9982 & -2.3895 \\
\hline 42 & $\mathrm{C}$ & 3.4702 & -7.2586 & -2.1855 & 6.8779 & 0.2561 & -3.9544 \\
\hline 43 & $\mathrm{C}$ & 0.8115 & -1.8926 & 2.9568 & 3.3069 & -0.3225 & 3.3603 \\
\hline 44 & $\mathrm{O}$ & 5.8223 & -1.4967 & 1.2419 & 3.7682 & 4.7023 & 0.9024 \\
\hline 45 & $\mathrm{C}$ & 4.5354 & -2.1443 & 1.2435 & 3.9433 & 3.2727 & 0.8395 \\
\hline 46 & $\mathrm{C}$ & 4.5530 & -3.2803 & 0.2171 & 4.7609 & 2.9384 & -0.4123 \\
\hline 47 & C & 4.9849 & -2.8066 & -1.1774 & 4.1126 & 3.5010 & -1.6820 \\
\hline 48 & $\mathrm{C}$ & 6.2691 & -1.9818 & -1.0919 & 3.8174 & 4.9870 & -1.5097 \\
\hline
\end{tabular}




\begin{tabular}{|c|c|c|c|c|c|c|c|}
\hline 49 & $\mathrm{C}$ & 6.1472 & -0.9076 & -0.0004 & 3.0631 & 5.2399 & -0.1948 \\
\hline 50 & $\mathrm{H}$ & 2.8163 & 0.4952 & 3.2650 & 2.9640 & 2.7834 & 0.7723 \\
\hline 51 & $\mathrm{H}$ & 5.2507 & -4.0648 & 0.5432 & 5.7676 & 3.3660 & -0.3122 \\
\hline 52 & $\mathrm{H}$ & 4.1912 & -2.1480 & -1.5617 & 3.1527 & 2.9810 & -1.8227 \\
\hline 53 & $\mathrm{H}$ & 7.0981 & -2.6526 & -0.8210 & 4.7681 & 5.5348 & -1.4570 \\
\hline 54 & $\mathrm{H}$ & 7.1140 & -0.4139 & 0.1441 & 3.0011 & 6.3169 & -0.0119 \\
\hline 55 & $\mathrm{C}$ & 4.2529 & -2.6204 & 2.6610 & 4.6337 & 2.8527 & 2.1197 \\
\hline 56 & $\mathrm{O}$ & 5.2023 & -3.9165 & -2.0245 & 4.9679 & 3.3185 & -2.7953 \\
\hline 57 & $\mathrm{O}$ & 6.5778 & -1.2916 & -2.2941 & 3.0138 & 5.5013 & -2.5672 \\
\hline 58 & $\mathrm{O}$ & 5.1692 & 0.0170 & -0.4069 & 1.7772 & 4.6767 & -0.3037 \\
\hline 59 & $\mathrm{H}$ & 3.3774 & -3.2742 & 2.6380 & 4.9846 & 1.8172 & 2.0406 \\
\hline 60 & $\mathrm{O}$ & 3.9407 & -1.5367 & 3.5246 & 3.7137 & 2.9825 & 3.1948 \\
\hline 61 & $\mathrm{H}$ & 5.1155 & -3.1934 & 3.0334 & 5.5076 & 3.5066 & 2.2791 \\
\hline 62 & $\mathrm{H}$ & 4.4545 & -4.5352 & -1.9070 & 5.2320 & 2.3764 & -2.8146 \\
\hline 63 & $\mathrm{C}$ & 7.1984 & -2.0791 & -3.3101 & 3.7490 & 6.0228 & -3.6776 \\
\hline 64 & $\mathrm{C}$ & 5.0456 & -1.0283 & 4.2662 & 4.3429 & 2.7625 & 4.4490 \\
\hline 65 & $\mathrm{O}$ & 4.9118 & 3.4982 & 0.7245 & -1.3127 & 5.4047 & 1.5290 \\
\hline 66 & $\mathrm{C}$ & 4.6265 & 2.0886 & 0.7668 & -0.1178 & 4.6640 & 1.2043 \\
\hline 67 & $\mathrm{C}$ & 5.4579 & 1.4129 & -0.3233 & 0.6683 & 5.4522 & 0.1549 \\
\hline 68 & $\mathrm{C}$ & 5.1334 & 1.9913 & -1.7074 & -0.1746 & 5.7771 & -1.0839 \\
\hline 69 & $\mathrm{C}$ & 5.2319 & 3.5183 & -1.6828 & -1.4874 & 6.4297 & -0.6748 \\
\hline 70 & $\mathrm{C}$ & 4.4831 & 4.1033 & -0.4722 & -2.1747 & 5.6027 & 0.4332 \\
\hline 71 & $\mathrm{H}$ & 3.5662 & 1.9223 & 0.5465 & -0.3944 & 3.6896 & 0.7857 \\
\hline 72 & $\mathrm{H}$ & 6.5257 & 1.5720 & -0.1166 & 1.0137 & 6.3941 & 0.6020 \\
\hline 73 & $\mathrm{H}$ & 4.0904 & 1.7255 & -1.9393 & -0.4095 & 4.8227 & -1.5795 \\
\hline 74 & $\mathrm{H}$ & 6.2937 & 3.7907 & -1.5845 & -1.2793 & 7.4340 & -0.2791 \\
\hline 75 & $\mathrm{H}$ & 4.7178 & 5.1688 & -0.3756 & -3.0404 & 6.1378 & 0.8349 \\
\hline 76 & $\mathrm{C}$ & 4.9441 & 1.5915 & 2.1666 & 0.6675 & 4.4806 & 2.4970 \\
\hline 77 & $\mathrm{O}$ & 6.0309 & 1.4871 & -2.6753 & 0.5286 & 6.6494 & -1.9457 \\
\hline 78 & $\mathrm{O}$ & 4.6784 & 4.1382 & -2.8344 & -2.3174 & 6.5243 & -1.8281 \\
\hline 79 & $\mathrm{O}$ & 3.1034 & 3.9146 & -0.6741 & -2.5687 & 4.3675 & -0.1237 \\
\hline 80 & $\mathrm{H}$ & 4.8895 & 0.4981 & 2.1623 & 1.7079 & 4.2237 & 2.2649 \\
\hline 81 & $\mathrm{O}$ & 4.0924 & 2.1413 & 3.1576 & 0.0889 & 3.5099 & 3.3619 \\
\hline 82 & $\mathrm{H}$ & 5.9638 & 1.8970 & 2.4281 & 0.6575 & 5.4331 & 3.0395 \\
\hline 83 & $\mathrm{H}$ & 6.0458 & 0.5135 & -2.5907 & 1.3646 & 6.2015 & -2.1907 \\
\hline 84 & $\mathrm{C}$ & 5.5217 & 4.1515 & -3.9856 & -3.4013 & 7.4433 & -1.6839 \\
\hline 85 & $\mathrm{C}$ & 2.7819 & 1.5854 & 3.1404 & 0.4126 & 2.1757 & 2.9817 \\
\hline 86 & $\mathrm{O}$ & 0.4040 & 5.8651 & 0.8821 & -5.6107 & 2.5543 & 0.8554 \\
\hline 87 & $\mathrm{C}$ & 1.3705 & 4.7960 & 0.8084 & -4.2623 & 3.0362 & 0.9980 \\
\hline 88 & $\mathrm{C}$ & 2.2170 & 5.0092 & -0.4494 & -3.9546 & 4.0274 & -0.1264 \\
\hline 89 & $\mathrm{C}$ & 1.3493 & 5.0904 & -1.7129 & -4.2240 & 3.4290 & -1.5099 \\
\hline 90 & $\mathrm{C}$ & 0.2330 & 6.1147 & -1.5263 & -5.6194 & 2.8129 & -1.5605 \\
\hline 91 & $\mathrm{C}$ & -0.5056 & 5.8836 & -0.1984 & -5.8426 & 1.8789 & -0.3610 \\
\hline 92 & $\mathrm{H}$ & 0.1730 & 2.3307 & 2.8370 & -3.5606 & 2.1970 & 0.9160 \\
\hline 93 & $\mathrm{H}$ & 2.7814 & 5.9477 & -0.3535 & -4.5750 & 4.9265 & -0.0080 \\
\hline 94 & $\mathrm{H}$ & 0.8803 & 4.1051 & -1.8573 & -3.4951 & 2.6199 & -1.6676 \\
\hline 95 & $\mathrm{H}$ & 0.6852 & 7.1170 & -1.4850 & -6.3665 & 3.6160 & -1.5009 \\
\hline 96 & $\mathrm{H}$ & -1.1791 & 6.7254 & -0.0074 & -6.8896 & 1.5624 & -0.3340 \\
\hline 97 & $\mathrm{C}$ & 2.1675 & 4.8084 & 2.1051 & -4.1609 & 3.6612 & 2.3825 \\
\hline 98 & $\mathrm{O}$ & 2.1390 & 5.4636 & -2.8235 & -4.1130 & 4.4238 & -2.5133 \\
\hline 99 & $\mathrm{O}$ & -0.7437 & 6.0644 & -2.5581 & -5.8220 & 2.0395 & -2.7385 \\
\hline 100 & $\mathrm{O}$ & -1.2270 & 4.6807 & -0.2860 & -4.9879 & 0.7687 & -0.5055 \\
\hline 101 & $\mathrm{H}$ & 3.0166 & 4.1258 & 2.0212 & -3.2398 & 4.2546 & 2.4482 \\
\hline 102 & $\mathrm{O}$ & 1.3790 & 4.3608 & 3.2003 & -4.1945 & 2.6748 & 3.4065 \\
\hline 103 & $\mathrm{H}$ & 2.5541 & 5.8235 & 2.2840 & -5.0236 & 4.3199 & 2.5329 \\
\hline 104 & $\mathrm{H}$ & 2.9119 & 4.8660 & -2.8538 & -3.2991 & 4.9354 & -2.3340 \\
\hline
\end{tabular}




\begin{tabular}{|c|c|c|c|c|c|c|c|}
\hline 105 & $\mathrm{C}$ & -0.4179 & 6.8009 & -3.7372 & -6.3618 & 2.7655 & -3.8458 \\
\hline 106 & $\mathrm{C}$ & 0.7356 & 5.3919 & 3.9366 & -2.9037 & 2.1316 & 3.6578 \\
\hline 107 & $\mathrm{O}$ & -4.1130 & 3.5016 & 1.6471 & -5.2408 & -2.5531 & 1.0232 \\
\hline 108 & $\mathrm{C}$ & -2.7261 & 3.5619 & 1.2645 & -4.7277 & -1.2167 & 0.8692 \\
\hline 109 & $\mathrm{C}$ & -2.5629 & 4.6706 & 0.2219 & -5.5235 & -0.5278 & -0.2387 \\
\hline 110 & $\mathrm{C}$ & -3.4943 & 4.4910 & -0.9811 & -5.4344 & -1.3103 & -1.5566 \\
\hline 111 & $\mathrm{C}$ & -4.9297 & 4.2986 & -0.4981 & -5.8165 & -2.7687 & -1.3319 \\
\hline 112 & $\mathrm{C}$ & -4.9864 & 3.2055 & 0.5839 & -5.0523 & -3.3523 & -0.1232 \\
\hline 113 & $\mathrm{H}$ & -2.8067 & -0.4727 & 2.7137 & -3.6775 & -1.2622 & 0.5614 \\
\hline 114 & $\mathrm{H}$ & -2.7984 & 5.6329 & 0.6988 & -6.5768 & -0.4576 & 0.0630 \\
\hline 115 & $\mathrm{H}$ & -3.1891 & 3.5764 & -1.5102 & -4.3838 & -1.2786 & -1.8846 \\
\hline 116 & $\mathrm{H}$ & -5.2811 & 5.2422 & -0.0541 & -6.8922 & -2.8187 & -1.1104 \\
\hline 117 & $\mathrm{H}$ & -5.9906 & 3.1697 & 1.0194 & -5.4415 & -4.3406 & 0.1394 \\
\hline 118 & $\mathrm{C}$ & -1.9139 & 3.8635 & 2.5171 & -4.8438 & -0.5208 & 2.2162 \\
\hline 119 & $\mathrm{O}$ & -3.4258 & 5.6283 & -1.8193 & -6.2970 & -0.7491 & -2.5253 \\
\hline 120 & $\mathrm{O}$ & -5.8229 & 3.8857 & -1.5218 & -5.5308 & -3.5026 & -2.5179 \\
\hline 121 & $\mathrm{O}$ & -4.6589 & 1.9710 & -0.0119 & -3.6860 & -3.4291 & -0.4637 \\
\hline 122 & $\mathrm{H}$ & -0.9294 & 4.2363 & 2.2157 & -4.5515 & 0.5290 & 2.0978 \\
\hline 123 & $\mathrm{O}$ & -1.7778 & 2.7604 & 3.3967 & -4.0834 & -1.1408 & 3.2492 \\
\hline 124 & $\mathrm{H}$ & -2.4327 & 4.6499 & 3.0768 & -5.8865 & -0.5526 & 2.5494 \\
\hline 125 & $\mathrm{H}$ & -2.5024 & 5.7043 & -2.1344 & -6.0548 & 0.1950 & -2.6179 \\
\hline 126 & $\mathrm{C}$ & -6.3087 & 4.9255 & -2.3701 & -6.2090 & -4.7589 & -2.5871 \\
\hline 127 & $\mathrm{C}$ & -0.7949 & 1.8316 & 2.9580 & -2.6974 & -1.2535 & 2.9562 \\
\hline 128 & $\mathrm{O}$ & -6.0459 & -1.2801 & 0.9973 & -1.3389 & -5.9222 & 0.8863 \\
\hline 129 & $\mathrm{C}$ & -5.1967 & -0.1234 & 1.1106 & -2.1226 & -4.7149 & 0.9014 \\
\hline 130 & $\mathrm{C}$ & -5.6052 & 0.9004 & 0.0455 & -3.0132 & -4.6816 & -0.3455 \\
\hline 131 & $\mathrm{C}$ & -5.6382 & 0.3045 & -1.3680 & -2.1903 & -4.8213 & -1.6284 \\
\hline 132 & $\mathrm{C}$ & -6.4257 & -1.0056 & -1.3787 & -1.2901 & -6.0494 & -1.5406 \\
\hline 133 & $\mathrm{C}$ & -5.9490 & -1.9285 & -0.2471 & -0.4821 & -6.0293 & -0.2320 \\
\hline 134 & $\mathrm{H}$ & -4.2424 & -2.7336 & 3.9994 & -1.4572 & -3.8428 & 0.8840 \\
\hline 135 & $\mathrm{H}$ & -6.6075 & 1.2899 & 0.2744 & -3.7379 & -5.5061 & -0.3012 \\
\hline 136 & $\mathrm{H}$ & -4.6002 & 0.0719 & -1.6511 & -1.5435 & -3.9350 & -1.7088 \\
\hline 137 & $\mathrm{H}$ & -7.4892 & -0.7763 & -1.2156 & -1.9155 & -6.9524 & -1.5320 \\
\hline 138 & $\mathrm{H}$ & -6.5994 & -2.8074 & -0.1843 & 0.0467 & -6.9796 & -0.1138 \\
\hline 139 & $\mathrm{C}$ & -5.3660 & 0.4152 & 2.5262 & -2.9271 & -4.7051 & 2.1841 \\
\hline 140 & $\mathrm{O}$ & -6.2393 & 1.2069 & -2.2751 & -3.0441 & -4.9365 & -2.7533 \\
\hline 141 & $\mathrm{O}$ & -6.2705 & -1.7463 & -2.5817 & -0.3591 & -6.1069 & -2.6163 \\
\hline 142 & $\mathrm{H}$ & -4.9616 & 1.4362 & 2.5720 & -3.6846 & -3.9121 & 2.1273 \\
\hline 143 & $\mathrm{O}$ & -4.7513 & -0.4025 & 3.5049 & -2.0500 & -4.4656 & 3.2730 \\
\hline 144 & $\mathrm{H}$ & -6.4377 & 0.4397 & 2.7564 & -3.4420 & -5.6725 & 2.3024 \\
\hline 145 & $\mathrm{H}$ & -5.8856 & 2.0996 & -2.0941 & -3.7001 & -4.2143 & -2.6963 \\
\hline 146 & $\mathrm{C}$ & -7.0585 & -1.3015 & -3.6849 & -0.8141 & -6.8342 & -3.7613 \\
\hline 147 & $\mathrm{C}$ & -3.3555 & -0.1562 & 3.6142 & -2.7655 & -4.2901 & 4.4881 \\
\hline 148 & $\mathrm{H}$ & -4.1536 & -0.4121 & 0.9379 & 0.5543 & -1.4681 & 3.7460 \\
\hline 149 & $\mathrm{H}$ & -2.9710 & -3.7838 & 4.7086 & 0.7315 & -1.8197 & 2.0109 \\
\hline 150 & $\mathrm{H}$ & -2.6500 & -2.8798 & 3.2111 & -0.4994 & -2.6952 & 2.9715 \\
\hline 151 & $\mathrm{H}$ & -2.7744 & -3.4556 & 0.9136 & 3.0649 & 0.3656 & 4.1723 \\
\hline 152 & $\mathrm{H}$ & 0.5458 & -0.8540 & 3.1732 & 2.8589 & 0.0676 & 2.4351 \\
\hline 153 & $\mathrm{H}$ & 1.2571 & -2.3491 & 3.8532 & 2.8874 & -1.3156 & 3.5643 \\
\hline 154 & $\mathrm{H}$ & 0.9252 & -3.3307 & 0.6356 & 3.5656 & 2.8251 & 5.2156 \\
\hline 155 & $\mathrm{H}$ & 4.7291 & -0.0681 & 4.6828 & 4.8172 & 1.7723 & 4.4847 \\
\hline 156 & $\mathrm{H}$ & 5.3187 & -1.7172 & 5.0801 & 5.1085 & 3.5299 & 4.6451 \\
\hline 157 & $\mathrm{H}$ & 3.7605 & -1.4230 & 0.9573 & -0.0413 & 1.5133 & 3.7245 \\
\hline 158 & $\mathrm{H}$ & 2.2333 & 2.0599 & 3.9547 & 0.0019 & 1.9193 & 1.9931 \\
\hline 159 & $\mathrm{H}$ & 2.2520 & 1.8216 & 2.2055 & 1.5008 & 2.0312 & 2.9662 \\
\hline 160 & $\mathrm{H}$ & 0.0905 & 6.0040 & 3.2959 & -3.0376 & 1.2844 & 4.3348 \\
\hline
\end{tabular}




\begin{tabular}{rrrrrrrr}
161 & $\mathrm{H}$ & 0.1265 & 4.8934 & 4.6958 & -2.4316 & 1.7717 & 2.7334 \\
162 & $\mathrm{H}$ & 1.4765 & 6.0420 & 4.4271 & -2.2309 & 2.8805 & 4.0968 \\
163 & $\mathrm{H}$ & 0.8468 & 3.8356 & 0.7265 & -2.1956 & -1.5100 & 3.8910 \\
164 & $\mathrm{H}$ & -0.7214 & 1.0602 & 3.7302 & -2.5007 & -2.0473 & 2.2268 \\
165 & $\mathrm{H}$ & -1.0799 & 1.3439 & 2.0129 & -2.2844 & -0.3122 & 2.5714 \\
166 & $\mathrm{H}$ & -2.4230 & 2.6045 & 0.8252 & -2.0309 & -4.0655 & 5.2661 \\
167 & $\mathrm{H}$ & -3.1585 & 0.9085 & 3.7928 & -3.4797 & -3.4576 & 4.4058 \\
168 & $\mathrm{H}$ & -2.9917 & -0.7509 & 4.4561 & -3.3156 & -5.2040 & 4.7601 \\
169 & $\mathrm{H}$ & -1.4069 & -5.8157 & -4.8355 & 6.7340 & -5.0187 & -2.7462 \\
170 & $\mathrm{H}$ & -2.9186 & -6.5452 & -4.2143 & 6.7790 & -4.6111 & -1.0135 \\
171 & $\mathrm{H}$ & -2.8681 & -4.7977 & -4.5860 & 6.3602 & -6.2741 & -1.5227 \\
172 & $\mathrm{H}$ & 4.3727 & -7.2029 & -2.8003 & 6.7244 & 0.9914 & -4.7482 \\
173 & $\mathrm{H}$ & 3.5183 & -8.1547 & -1.5490 & 7.9467 & 0.2096 & -3.7022 \\
174 & $\mathrm{H}$ & 2.5764 & -7.3141 & -2.8125 & 6.5399 & -0.7315 & -4.2817 \\
175 & $\mathrm{H}$ & 7.4824 & -1.3840 & -4.1049 & 3.0119 & 6.4378 & -4.3693 \\
176 & $\mathrm{H}$ & 8.1003 & -2.5707 & -2.9164 & 4.4315 & 6.8193 & -3.3496 \\
177 & $\mathrm{H}$ & 6.5143 & -2.8426 & -3.6903 & 4.3228 & 5.2293 & -4.1648 \\
178 & $\mathrm{H}$ & 5.0073 & 4.7587 & -4.7354 & -3.9155 & 7.4749 & -2.6465 \\
179 & $\mathrm{H}$ & 6.4912 & 4.6131 & -3.7459 & -4.1119 & 7.1179 & -0.9131 \\
180 & $\mathrm{H}$ & 5.6931 & 3.1385 & -4.3600 & -3.0264 & 8.4428 & -1.4280 \\
181 & $\mathrm{H}$ & -1.2964 & 6.7459 & -4.3856 & -6.5265 & 2.0370 & -4.6433 \\
182 & $\mathrm{H}$ & -0.2128 & 7.8535 & -3.4905 & -7.3194 & 3.2308 & -3.5727 \\
183 & $\mathrm{H}$ & 0.4559 & 6.3708 & -4.2337 & -5.6632 & 3.5392 & -4.1775 \\
184 & $\mathrm{H}$ & -7.0560 & 4.4634 & -3.0209 & -5.9799 & -5.1851 & -3.5658 \\
185 & $\mathrm{H}$ & -6.7853 & 5.7152 & -1.7707 & -5.8614 & -5.4511 & -1.8094 \\
186 & $\mathrm{H}$ & -5.4993 & 5.3648 & -2.9594 & -7.2930 & -4.6172 & -2.4869 \\
187 & $\mathrm{H}$ & -6.9239 & -2.0452 & -4.4751 & 0.0181 & -6.8485 & -4.4692 \\
188 & $\mathrm{H}$ & -8.1208 & -1.2572 & -3.4027 & -1.0771 & -7.8647 & -3.4849 \\
189 & $\mathrm{H}$ & -6.7406 & -0.3124 & -4.0260 & -1.6834 & -6.3429 & -4.2075 \\
\hline & & & & & & & \\
\hline
\end{tabular}


Table S10. Cartesian coordinates of atoms in the DM- $\beta-C D$ structures ZULQAY in vacuo and BOYFOK03 in water, obtained after the M062X-GD3/6-31++G(d,p) re-optimization of the structures given in Table S9. The true minima are confirmed by zero imaginary frequencies.

\begin{tabular}{|c|c|c|c|c|c|c|c|c|}
\hline \multirow[b]{2}{*}{ No. } & \multirow[b]{2}{*}{ Atom } & \multicolumn{3}{|c|}{ ZULQAY } & \multirow[b]{2}{*}{ Atom } & \multicolumn{3}{|c|}{ BOYFOK03 } \\
\hline & & $\mathbf{x}$ & $\mathbf{y}$ & $\mathbf{Z}$ & & $\mathbf{x}$ & $\mathbf{y}$ & $\mathbf{Z}$ \\
\hline 1 & $\mathrm{C}$ & 4.5160 & 3.9893 & -0.4803 & $\mathrm{C}$ & -2.6578 & 5.4732 & $\begin{array}{l}-0.1770 \\
\end{array}$ \\
\hline 2 & $\mathrm{C}$ & 5.0281 & 3.3625 & -1.7803 & $\mathrm{C}$ & -1.9691 & 5.9896 & -1.4452 \\
\hline 3 & $\mathrm{C}$ & 4.7939 & 1.8582 & -1.7703 & $\mathrm{C}$ & -0.6096 & 5.3256 & -1.6207 \\
\hline 4 & $\mathrm{C}$ & 5.3592 & 1.2668 & -0.4836 & $\mathrm{C}$ & 0.1791 & 5.4678 & -0.3256 \\
\hline 5 & $\mathrm{C}$ & 4.8593 & 1.9986 & 0.7585 & $\mathrm{C}$ & -0.6056 & 4.9143 & 0.8602 \\
\hline 6 & $\mathrm{C}$ & 5.5202 & 1.4875 & 2.0316 & $\mathrm{C}$ & 0.1440 & 5.0547 & 2.1668 \\
\hline 7 & $\mathrm{C}$ & 5.0075 & 3.9550 & -4.0974 & $\mathrm{C}$ & -2.7836 & 6.6841 & -3.5773 \\
\hline 8 & $\mathrm{C}$ & 7.6661 & 2.4896 & 1.8706 & $\mathrm{C}$ & 1.5337 & 6.4639 & 3.3903 \\
\hline 9 & $\mathrm{O}$ & 4.3606 & 4.0228 & -2.8343 & $\mathrm{O}$ & -2.8389 & 5.7259 & -2.5280 \\
\hline 10 & $\mathrm{O}$ & 5.4271 & 1.2836 & -2.8857 & $\mathrm{O}$ & 0.0750 & 5.9419 & -2.6857 \\
\hline 11 & $\mathrm{O}$ & 4.9377 & -0.0848 & -0.4578 & $\mathrm{O}$ & 1.4009 & 4.7739 & -0.5094 \\
\hline 12 & $\mathrm{O}$ & 5.1233 & 3.3995 & 0.6411 & $\mathrm{O}$ & -1.8356 & 5.6390 & 0.9516 \\
\hline 13 & $\mathrm{O}$ & 6.9113 & 1.2970 & 1.9235 & $\mathrm{O}$ & 0.6961 & 6.3469 & 2.2576 \\
\hline 14 & $\mathrm{C}$ & -0.2803 & 5.9717 & 0.3819 & $\mathrm{H}$ & -3.5650 & 6.0543 & 0.0187 \\
\hline 15 & $\mathrm{C}$ & 0.5915 & 6.5333 & -0.7459 & $\mathrm{H}$ & -1.8201 & 7.0734 & -1.3339 \\
\hline 16 & $\mathrm{C}$ & 1.6000 & 5.4839 & -1.1896 & $\mathrm{H}$ & -0.7719 & 4.2537 & -1.8173 \\
\hline 17 & $\mathrm{C}$ & 2.3488 & 4.9251 & 0.0191 & $\mathrm{H}$ & 0.3722 & 6.5326 & -0.1458 \\
\hline 18 & $\mathrm{C}$ & 1.3932 & 4.4584 & 1.1127 & $\mathrm{H}$ & -0.8189 & 3.8472 & 0.6968 \\
\hline 19 & $\mathrm{C}$ & 2.1182 & 4.0778 & 2.3829 & $\mathrm{H}$ & 0.9446 & 4.3029 & 2.2064 \\
\hline 20 & $\mathrm{C}$ & 0.1949 & 7.9191 & -2.6508 & $\mathrm{H}$ & -3.5158 & 6.3734 & -4.3233 \\
\hline 21 & $\mathrm{C}$ & 1.8106 & 3.0074 & 4.4349 & $\mathrm{H}$ & -3.0490 & 7.6804 & -3.2043 \\
\hline 22 & $\mathrm{O}$ & -0.2933 & 6.9147 & -1.7763 & $\mathrm{H}$ & -1.7866 & 6.7143 & -4.0219 \\
\hline 23 & $\mathrm{O}$ & 2.5133 & 6.0589 & -2.0923 & $\mathrm{H}$ & 1.9830 & 7.4579 & 3.3632 \\
\hline 24 & $\mathrm{O}$ & 3.1267 & 3.8314 & -0.4422 & $\mathrm{H}$ & 0.9559 & 6.3569 & 4.3189 \\
\hline 25 & $\mathrm{O}$ & 0.5130 & 5.5255 & 1.4490 & $\mathrm{H}$ & 2.3188 & 5.6991 & 3.3656 \\
\hline 26 & $\mathrm{O}$ & 1.2201 & 3.3876 & 3.2176 & $\mathrm{H}$ & 1.0132 & 5.6842 & -2.6223 \\
\hline 27 & $\mathrm{C}$ & -4.9270 & 3.5661 & 0.1904 & $\mathrm{C}$ & 2.5849 & 5.4139 & -0.1090 \\
\hline 28 & $\mathrm{C}$ & -4.6402 & 4.5370 & -0.9612 & $\mathrm{C}$ & 3.5875 & 5.2736 & -1.2603 \\
\hline 29 & $\mathrm{C}$ & -3.1408 & 4.6394 & -1.2161 & $\mathrm{C}$ & 3.9848 & 3.8139 & -1.4186 \\
\hline 30 & $\mathrm{C}$ & -2.4378 & 4.9223 & 0.1064 & $\mathrm{C}$ & 4.4502 & 3.2516 & -0.0776 \\
\hline 31 & $\mathrm{C}$ & -2.8003 & 3.8842 & 1.1631 & $\mathrm{C}$ & 3.3812 & 3.4661 & 0.9926 \\
\hline 32 & $\mathrm{C}$ & -2.1215 & 4.1446 & 2.4911 & $\mathrm{C}$ & 3.8322 & 3.0299 & 2.3749 \\
\hline 33 & $\mathrm{C}$ & -5.6335 & 5.0252 & -3.0808 & $\mathrm{C}$ & 3.8322 & 6.3528 & -3.3766 \\
\hline 34 & $\mathrm{C}$ & -1.4164 & 5.8161 & 3.9418 & $\mathrm{C}$ & 1.9565 & 1.8925 & 3.2409 \\
\hline 35 & $\mathrm{O}$ & -5.3698 & 4.0628 & -2.0711 & $\mathrm{O}$ & 2.9536 & 5.7873 & -2.4134 \\
\hline 36 & $\mathrm{O}$ & -2.9006 & 5.6825 & -2.1274 & $\mathrm{O}$ & 5.0070 & 3.6950 & -2.3796 \\
\hline 37 & $\mathrm{O}$ & -1.0459 & 4.9311 & -0.1622 & $\mathrm{O}$ & 4.6959 & 1.8723 & -0.3005 \\
\hline 38 & $\mathrm{O}$ & -4.2176 & 3.9257 & 1.3463 & $\mathrm{O}$ & 3.0949 & 4.8612 & 1.0745 \\
\hline 39 & $\mathrm{O}$ & -2.1648 & 5.5201 & 2.7847 & $\mathrm{O}$ & 2.7792 & 3.0371 & 3.3161 \\
\hline 40 & $\mathrm{C}$ & -5.9003 & -1.6235 & -0.0558 & $\mathrm{H}$ & 2.3952 & 6.4685 & 0.1097 \\
\hline 41 & $\mathrm{C}$ & -6.5235 & -0.7531 & -1.1510 & $\mathrm{H}$ & 4.4805 & 5.8688 & -1.0196 \\
\hline 42 & $\mathrm{C}$ & -5.7211 & 0.5286 & -1.3328 & $\mathrm{H}$ & 3.0883 & 3.2523 & -1.7249 \\
\hline 43 & $\mathrm{C}$ & -5.4695 & 1.2016 & 0.0171 & $\mathrm{H}$ & 5.3779 & 3.7623 & 0.2227 \\
\hline 44 & $\mathrm{C}$ & -4.8702 & 0.2167 & 1.0183 & $\mathrm{H}$ & 2.4754 & 2.9176 & 0.6962 \\
\hline 45 & $\mathrm{C}$ & -4.7155 & 0.7986 & 2.4119 & $\mathrm{H}$ & 4.5819 & 3.7437 & 2.7326 \\
\hline 46 & $\mathrm{C}$ & -7.5128 & -1.1694 & -3.2857 & $\mathrm{H}$ & 4.2958 & 2.0354 & 2.3138 \\
\hline 47 & $\mathrm{C}$ & -2.5893 & 0.0128 & 3.0603 & $\mathrm{H}$ & 3.2051 & 6.7503 & -4.1754 \\
\hline 48 & $\mathrm{O}$ & -6.5557 & -1.5542 & -2.3121 & $\mathrm{H}$ & 4.4122 & 7.1713 & -2.9340 \\
\hline
\end{tabular}




\begin{tabular}{|c|c|c|c|c|c|c|c|c|}
\hline 49 & $\mathrm{O}$ & -6.4246 & 1.3987 & -2.1821 & $\mathrm{H}$ & 4.5135 & 5.5985 & -3.7762 \\
\hline 50 & $\mathrm{O}$ & -4.5834 & 2.2787 & -0.2447 & $\mathrm{H}$ & 1.2339 & 1.9596 & 4.0563 \\
\hline 51 & $\mathrm{O}$ & -5.7506 & -0.8975 & 1.1355 & $\mathrm{H}$ & 1.4094 & 1.8314 & 2.2893 \\
\hline 52 & $\mathrm{O}$ & -3.9796 & -0.0411 & 3.2757 & $\mathrm{H}$ & 2.5469 & 0.9736 & 3.3599 \\
\hline 53 & $\mathrm{C}$ & -2.4721 & -5.5643 & -0.3315 & $\mathrm{H}$ & 5.2671 & 2.7559 & -2.4043 \\
\hline 54 & $\mathrm{C}$ & -3.2811 & -5.3349 & -1.6137 & $\mathrm{C}$ & 5.9202 & 1.3221 & 0.1067 \\
\hline 55 & $\mathrm{C}$ & -3.7151 & -3.8773 & -1.7203 & $\mathrm{C}$ & 6.4650 & 0.4942 & -1.0702 \\
\hline 56 & $\mathrm{C}$ & -4.3709 & -3.4592 & -0.4096 & $\mathrm{C}$ & 5.5544 & -0.6934 & -1.3368 \\
\hline 57 & $\mathrm{C}$ & -3.4611 & -3.7366 & 0.7816 & $\mathrm{C}$ & 5.3396 & -1.4493 & -0.0313 \\
\hline 58 & $\mathrm{C}$ & -4.0823 & -3.3376 & 2.1031 & $\mathrm{C}$ & 4.8187 & -0.5341 & 1.0726 \\
\hline 59 & $\mathrm{C}$ & -3.1520 & -6.0807 & -3.8787 & $\mathrm{C}$ & 4.6402 & -1.2564 & 2.3899 \\
\hline 60 & $\mathrm{C}$ & -6.0775 & -3.2351 & 3.2874 & $\mathrm{C}$ & 7.7075 & 2.1017 & -2.2983 \\
\hline 61 & $\mathrm{O}$ & -2.4615 & -5.7582 & -2.6811 & $\mathrm{C}$ & 5.5626 & -2.8725 & 3.7860 \\
\hline 62 & $\mathrm{O}$ & -4.6290 & -3.7386 & -2.7801 & $\mathrm{O}$ & 6.5513 & 1.2773 & -2.2434 \\
\hline 63 & $\mathrm{O}$ & -4.6630 & -2.0780 & -0.5343 & $\mathrm{O}$ & 6.1553 & -1.5316 & -2.2929 \\
\hline 64 & $\mathrm{O}$ & -3.1769 & -5.1370 & 0.8050 & $\mathrm{O}$ & 4.4275 & -2.4955 & -0.3136 \\
\hline 65 & $\mathrm{O}$ & -5.4336 & -3.7268 & 2.1330 & $\mathrm{O}$ & 5.7681 & 0.5214 & 1.2517 \\
\hline 66 & $\mathrm{C}$ & 2.7457 & -5.2684 & 0.3217 & $\mathrm{O}$ & 5.7653 & -2.0652 & 2.6436 \\
\hline 67 & $\mathrm{C}$ & 2.1977 & -6.1723 & -0.7954 & $\mathrm{H}$ & 6.6214 & 2.1136 & 0.3921 \\
\hline 68 & $\mathrm{C}$ & 0.8219 & -5.6895 & -1.2205 & $\mathrm{H}$ & 7.4606 & 0.1151 & -0.7968 \\
\hline 69 & $\mathrm{C}$ & -0.0761 & -5.5016 & 0.0025 & $\mathrm{H}$ & 4.5809 & -0.3157 & -1.6891 \\
\hline 70 & $\mathrm{C}$ & 0.5878 & -4.6483 & 1.0790 & $\mathrm{H}$ & 6.3023 & -1.8645 & 0.2916 \\
\hline 71 & $\mathrm{C}$ & -0.2061 & -4.6096 & 2.3642 & $\mathrm{H}$ & 3.8485 & -0.1082 & 0.7785 \\
\hline 72 & $\mathrm{C}$ & 4.2160 & -6.9333 & -1.7769 & $\mathrm{H}$ & 3.7367 & -1.8785 & 2.3437 \\
\hline 73 & $\mathrm{C}$ & -0.3302 & -3.5180 & 4.4239 & $\mathrm{H}$ & 4.5074 & -0.5114 & 3.1918 \\
\hline 74 & $\mathrm{O}$ & 3.0491 & -6.1481 & -1.9210 & $\mathrm{H}$ & 7.7001 & 2.5906 & -3.2729 \\
\hline 75 & $\mathrm{O}$ & 0.2481 & -6.6267 & -2.0898 & $\mathrm{H}$ & 7.6955 & 2.8727 & -1.5191 \\
\hline 76 & $\mathrm{O}$ & -1.2600 & -4.8768 & -0.4659 & $\mathrm{H}$ & 8.6164 & 1.4988 & -2.1983 \\
\hline 77 & $\mathrm{O}$ & 1.8544 & -5.2134 & 1.4025 & $\mathrm{H}$ & 6.4312 & -3.5256 & 3.8836 \\
\hline 78 & $\mathrm{O}$ & 0.3158 & -3.5850 & 3.1780 & $\mathrm{H}$ & 5.4736 & -2.2567 & 4.6918 \\
\hline 79 & $\mathrm{C}$ & 5.8398 & -1.0648 & 0.0014 & $\mathrm{H}$ & 4.6539 & -3.4752 & 3.6717 \\
\hline 80 & C & 5.9968 & -2.0908 & -1.1297 & $\mathrm{H}$ & 5.6980 & -2.3913 & -2.2537 \\
\hline 81 & $\mathrm{C}$ & 4.6854 & -2.8367 & -1.3424 & $\mathrm{C}$ & 4.7225 & -3.7737 & 0.1879 \\
\hline 82 & $\mathrm{C}$ & 4.2011 & -3.3710 & -0.0012 & $\mathrm{C}$ & 4.5117 & -4.7687 & -0.9569 \\
\hline 83 & $\mathrm{C}$ & 4.0786 & -2.2559 & 1.0300 & $\mathrm{C}$ & 3.0355 & -4.8376 & -1.3192 \\
\hline 84 & C & 3.6072 & -2.7651 & 2.3752 & $\mathrm{C}$ & 2.1886 & -5.0645 & -0.0681 \\
\hline 85 & $\mathrm{C}$ & 7.0646 & -2.1109 & -3.2674 & $\mathrm{C}$ & 2.5158 & -4.0438 & 1.0194 \\
\hline 86 & C & 3.7471 & -4.5501 & 3.8567 & $\mathrm{C}$ & 1.8081 & -4.3438 & 2.3283 \\
\hline 87 & $\mathrm{O}$ & 6.4102 & -1.3536 & -2.2611 & $\mathrm{C}$ & 5.7791 & -5.3428 & -2.8987 \\
\hline 88 & $\mathrm{O}$ & 4.8802 & -3.9031 & -2.2420 & $\mathrm{C}$ & 1.0609 & -2.2171 & 3.0326 \\
\hline 89 & $\mathrm{O}$ & 2.9564 & -4.0020 & -0.2401 & $\mathrm{O}$ & 5.3113 & -4.3191 & -2.0319 \\
\hline 90 & $\mathrm{O}$ & 5.3678 & -1.6585 & 1.1787 & $\mathrm{O}$ & 2.8253 & -5.8777 & -2.2448 \\
\hline 91 & $\mathrm{O}$ & 4.2732 & -3.9659 & 2.6871 & $\mathrm{O}$ & 0.8325 & -4.9436 & -0.4765 \\
\hline 92 & $\mathrm{H}$ & 4.7845 & 5.0512 & -0.4510 & $\mathrm{O}$ & 3.9166 & -4.0951 & 1.2880 \\
\hline 93 & $\mathrm{H}$ & 6.1126 & 3.5472 & -1.8461 & $\mathrm{O}$ & 1.9402 & -3.3000 & 3.2724 \\
\hline 94 & $\mathrm{H}$ & 3.7067 & 1.6827 & -1.7844 & $\mathrm{H}$ & 5.7544 & -3.8143 & 0.5482 \\
\hline 95 & $\mathrm{H}$ & 6.4557 & 1.3233 & -0.5293 & $\mathrm{H}$ & 4.8477 & -5.7606 & -0.6206 \\
\hline 96 & $\mathrm{H}$ & 3.7742 & 1.8352 & 0.8388 & $\mathrm{H}$ & 2.7477 & -3.8624 & -1.7425 \\
\hline 97 & $\mathrm{H}$ & 5.1063 & 0.5023 & 2.2631 & $\mathrm{H}$ & 2.3832 & -6.0769 & 0.3163 \\
\hline 98 & $\mathrm{H}$ & 5.2846 & 2.1855 & 2.8509 & $\mathrm{H}$ & 2.2444 & -3.0427 & 0.6552 \\
\hline 99 & $\mathrm{H}$ & 5.0098 & 2.9342 & -4.4822 & $\mathrm{H}$ & 2.2793 & -5.2284 & 2.7711 \\
\hline 100 & $\mathrm{H}$ & 6.0419 & 4.3135 & -4.0188 & $\mathrm{H}$ & 0.7507 & -4.5677 & 2.1429 \\
\hline 101 & $\mathrm{H}$ & 4.4497 & 4.6138 & -4.7646 & $\mathrm{H}$ & 6.4245 & -4.8595 & -3.6331 \\
\hline 102 & $\mathrm{H}$ & 7.3514 & 3.1893 & 2.6564 & $\mathrm{H}$ & 6.3634 & -6.0832 & -2.3392 \\
\hline 103 & $\mathrm{H}$ & 7.5673 & 2.9985 & 0.9051 & $\mathrm{H}$ & 4.9475 & -5.8411 & -3.4015 \\
\hline 104 & $\mathrm{H}$ & 8.7092 & 2.2116 & 2.0319 & $\mathrm{H}$ & 1.1505 & -1.5379 & 3.8831 \\
\hline
\end{tabular}




\begin{tabular}{|c|c|c|c|c|c|c|c|c|}
\hline 105 & $\mathrm{H}$ & 5.5230 & 0.3291 & -2.7150 & $\mathrm{H}$ & 1.3245 & -1.6631 & 2.1202 \\
\hline 106 & $\mathrm{H}$ & -0.9283 & 6.7486 & 0.7972 & $\mathrm{H}$ & 0.0258 & -2.5710 & 2.9509 \\
\hline 107 & $\mathrm{H}$ & 1.1333 & 7.4131 & -0.3649 & $\mathrm{H}$ & 1.8728 & -5.8971 & -2.4539 \\
\hline 108 & $\mathrm{H}$ & 1.0422 & 4.6551 & -1.6512 & $\mathrm{C}$ & -0.0029 & -6.0588 & -0.3394 \\
\hline 109 & $\mathrm{H}$ & 3.0001 & 5.7144 & 0.4251 & $\mathrm{C}$ & -0.8608 & -6.1598 & -1.6050 \\
\hline 110 & $\mathrm{H}$ & 0.8210 & 3.5928 & 0.7510 & $\mathrm{C}$ & -1.8218 & -4.9841 & -1.6733 \\
\hline 111 & $\mathrm{H}$ & 2.4810 & 4.9958 & 2.8738 & $\mathrm{C}$ & -2.6065 & -4.8777 & -0.3687 \\
\hline 112 & $\mathrm{H}$ & 2.9910 & 3.4542 & 2.1378 & $\mathrm{C}$ & -1.6540 & -4.8078 & 0.8247 \\
\hline 113 & $\mathrm{H}$ & 1.0643 & 7.5678 & -3.2097 & $\mathrm{C}$ & -2.3703 & -4.7982 & 2.1556 \\
\hline 114 & $\mathrm{H}$ & 0.4682 & 8.8198 & -2.0856 & $\mathrm{C}$ & -0.4357 & -6.9812 & -3.8033 \\
\hline 115 & $\mathrm{H}$ & -0.6233 & 8.1607 & -3.3308 & $\mathrm{C}$ & -2.0083 & -4.4408 & 4.4418 \\
\hline 116 & $\mathrm{H}$ & 2.1541 & 3.8821 & 5.0051 & $\mathrm{O}$ & 0.0168 & -6.1884 & -2.7123 \\
\hline 117 & $\mathrm{H}$ & 2.6698 & 2.3404 & 4.2702 & $\mathrm{O}$ & -2.6978 & -5.1535 & -2.7616 \\
\hline 118 & $\mathrm{H}$ & 1.0539 & 2.4774 & 5.0152 & $\mathrm{O}$ & -3.3879 & -3.7006 & -0.4888 \\
\hline 119 & $\mathrm{H}$ & 3.0770 & 5.3430 & -2.4375 & $\mathrm{O}$ & -0.8106 & -5.9604 & 0.8056 \\
\hline 120 & $\mathrm{H}$ & -5.9889 & 3.6101 & 0.4574 & $\mathrm{O}$ & -1.4226 & -4.5262 & 3.1616 \\
\hline 121 & $\mathrm{H}$ & -5.0061 & 5.5336 & -0.6682 & $\mathrm{H}$ & 0.5881 & -6.9722 & -0.2158 \\
\hline 122 & $\mathrm{H}$ & -2.7887 & 3.6706 & -1.6055 & $\mathrm{H}$ & -1.4374 & -7.0945 & -1.5519 \\
\hline 123 & $\mathrm{H}$ & -2.7590 & 5.9060 & 0.4703 & $\mathrm{H}$ & -1.2259 & -4.0645 & -1.7837 \\
\hline 124 & $\mathrm{H}$ & -2.5080 & 2.8822 & 0.8154 & $\mathrm{H}$ & -3.2560 & -5.7610 & -0.2701 \\
\hline 125 & $\mathrm{H}$ & -1.0779 & 3.8035 & 2.4394 & $\mathrm{H}$ & -1.0446 & -3.8957 & 0.7420 \\
\hline 126 & $\mathrm{H}$ & -2.6383 & 3.5633 & 3.2734 & $\mathrm{H}$ & -2.8463 & -5.7781 & 2.3220 \\
\hline 127 & $\mathrm{H}$ & -4.7152 & 5.3312 & -3.5849 & $\mathrm{H}$ & -3.1572 & -4.0302 & 2.1405 \\
\hline 128 & $\mathrm{H}$ & -6.1174 & 5.9106 & -2.6491 & $\mathrm{H}$ & 0.3369 & -6.9267 & -4.5708 \\
\hline 129 & $\mathrm{H}$ & -6.3177 & 4.5512 & -3.7863 & $\mathrm{H}$ & -0.5646 & -8.0251 & -3.4940 \\
\hline 130 & $\mathrm{H}$ & -0.3821 & 5.4668 & 3.8311 & $\mathrm{H}$ & -1.3794 & -6.5957 & -4.1951 \\
\hline 131 & $\mathrm{H}$ & -1.8638 & 5.3477 & 4.8306 & $\mathrm{H}$ & -1.2104 & -4.2098 & 5.1489 \\
\hline 132 & $\mathrm{H}$ & -1.4280 & 6.9000 & 4.0693 & $\mathrm{H}$ & -2.7657 & -3.6462 & 4.4800 \\
\hline 133 & $\mathrm{H}$ & -1.9520 & 5.8994 & -2.0900 & $\mathrm{H}$ & -2.4820 & -5.3894 & 4.7268 \\
\hline 134 & $\mathrm{H}$ & -6.5506 & -2.4681 & 0.1872 & $\mathrm{H}$ & -3.3627 & -4.4428 & -2.7107 \\
\hline 135 & $\mathrm{H}$ & -7.5472 & -0.4856 & -0.8450 & $\mathrm{C}$ & -4.7441 & -3.7365 & -0.1287 \\
\hline 136 & $\mathrm{H}$ & -4.7405 & 0.2539 & -1.7537 & $\mathrm{C}$ & -5.5468 & -3.1542 & -1.3093 \\
\hline 137 & $\mathrm{H}$ & -6.4274 & 1.5800 & 0.4065 & $\mathrm{C}$ & -5.2317 & -1.6760 & -1.4696 \\
\hline 138 & $\mathrm{H}$ & -3.8962 & -0.1177 & 0.6338 & $\mathrm{C}$ & -5.4189 & -0.9863 & -0.1247 \\
\hline 139 & $\mathrm{H}$ & -5.7124 & 0.9013 & 2.8535 & $\mathrm{C}$ & -4.5626 & -1.6423 & 0.9525 \\
\hline 140 & $\mathrm{H}$ & -4.2575 & 1.7954 & 2.3473 & $\mathrm{C}$ & -4.7135 & -0.9819 & 2.3039 \\
\hline 141 & $\mathrm{H}$ & -7.2750 & -0.1928 & -3.7120 & $\mathrm{C}$ & -5.8263 & -5.0988 & -2.6451 \\
\hline 142 & $\mathrm{H}$ & -8.5169 & -1.1329 & -2.8434 & $\mathrm{C}$ & -6.2504 & 0.0001 & 3.7497 \\
\hline 143 & $\mathrm{H}$ & -7.4918 & -1.9386 & -4.0592 & $\mathrm{O}$ & -5.2296 & -3.8159 & -2.5156 \\
\hline 144 & $\mathrm{H}$ & -2.2189 & 1.0480 & 3.0978 & $\mathrm{O}$ & -6.0918 & -1.1110 & -2.4300 \\
\hline 145 & $\mathrm{H}$ & -2.2938 & -0.4255 & 2.0965 & $\mathrm{O}$ & -5.0780 & 0.3729 & -0.3192 \\
\hline 146 & $\mathrm{H}$ & -2.1170 & -0.5624 & 3.8589 & $\mathrm{O}$ & -4.9778 & -3.0087 & 1.0493 \\
\hline 147 & $\mathrm{H}$ & -5.9320 & 2.2385 & -2.2210 & $\mathrm{O}$ & -6.0790 & -0.7599 & 2.5704 \\
\hline 148 & $\mathrm{H}$ & -2.2930 & -6.6367 & -0.1959 & $\mathrm{H}$ & -5.0564 & -4.7627 & 0.0938 \\
\hline 149 & $\mathrm{H}$ & -4.1871 & -5.9592 & -1.5615 & $\mathrm{H}$ & -6.6191 & -3.2636 & -1.0895 \\
\hline 150 & $\mathrm{H}$ & -2.8152 & -3.2613 & -1.8777 & $\mathrm{H}$ & -4.1772 & -1.5756 & -1.7729 \\
\hline 151 & $\mathrm{H}$ & -5.2970 & -4.0332 & -0.2805 & $\mathrm{H}$ & -6.4720 & -1.0743 & 0.1727 \\
\hline 152 & $\mathrm{H}$ & -2.5226 & -3.1753 & 0.6590 & $\mathrm{H}$ & -3.5025 & -1.6009 & 0.6671 \\
\hline 153 & $\mathrm{H}$ & -4.0068 & -2.2488 & 2.2279 & $\mathrm{H}$ & -4.1725 & -0.0254 & 2.2994 \\
\hline 154 & $\mathrm{H}$ & -3.5199 & -3.8220 & 2.9192 & $\mathrm{H}$ & -4.2629 & -1.6294 & 3.0733 \\
\hline 155 & $\mathrm{H}$ & -3.6074 & -5.1933 & -4.3219 & $\mathrm{H}$ & -5.6129 & -5.4449 & -3.6566 \\
\hline 156 & $\mathrm{H}$ & -3.9342 & -6.8256 & -3.6834 & $\mathrm{H}$ & -5.4040 & -5.8184 & -1.9338 \\
\hline 157 & $\mathrm{H}$ & -2.4113 & -6.5081 & -4.5561 & $\mathrm{H}$ & -6.9102 & -5.0377 & -2.4997 \\
\hline 158 & $\mathrm{H}$ & -5.9790 & -2.1443 & 3.3497 & $\mathrm{H}$ & -7.3189 & 0.1882 & 3.8654 \\
\hline 159 & $\mathrm{H}$ & -5.6553 & -3.6872 & 4.1965 & $\mathrm{H}$ & -5.8857 & -0.5496 & 4.6283 \\
\hline 160 & $\mathrm{H}$ & -7.1316 & -3.5076 & 3.2133 & $\mathrm{H}$ & -5.7122 & 0.9523 & 3.6724 \\
\hline
\end{tabular}




\begin{tabular}{rlrrrrrrr}
161 & $\mathrm{H}$ & -5.0831 & -2.8844 & -2.6680 & $\mathrm{H}$ & -5.9813 & -0.1450 & -2.3721 \\
162 & $\mathrm{H}$ & 3.6779 & -5.6567 & 0.7437 & $\mathrm{C}$ & -5.9218 & 1.3467 & 0.2379 \\
163 & $\mathrm{H}$ & 2.1073 & -7.1997 & -0.4094 & $\mathrm{C}$ & -6.2059 & 2.3790 & -0.8654 \\
164 & $\mathrm{H}$ & 0.9419 & -4.7053 & -1.7001 & $\mathrm{C}$ & -4.9226 & 3.1063 & -1.2273 \\
165 & $\mathrm{H}$ & -0.3056 & -6.4942 & 0.4197 & $\mathrm{C}$ & -4.2379 & 3.6414 & 0.0298 \\
166 & $\mathrm{H}$ & 0.7149 & -3.6235 & 0.7029 & $\mathrm{C}$ & -4.0661 & 2.5517 & 1.0847 \\
167 & $\mathrm{H}$ & -0.1171 & -5.5877 & 2.8649 & $\mathrm{C}$ & -3.5532 & 3.0890 & 2.4087 \\
168 & $\mathrm{H}$ & -1.2687 & -4.4363 & 2.1428 & $\mathrm{C}$ & -8.0546 & 1.3306 & -1.9235 \\
169 & $\mathrm{H}$ & 3.9592 & -7.9693 & -1.5240 & $\mathrm{C}$ & -1.8392 & 1.5913 & 3.0085 \\
170 & $\mathrm{H}$ & 4.8937 & -6.5284 & -1.0143 & $\mathrm{O}$ & -6.7028 & 1.7509 & -2.0296 \\
171 & $\mathrm{H}$ & 4.7311 & -6.9120 & -2.7379 & $\mathrm{O}$ & -5.2156 & 4.1612 & -2.1085 \\
172 & $\mathrm{H}$ & -0.2057 & -4.4510 & 4.9918 & $\mathrm{O}$ & -2.9695 & 4.1241 & -0.3890 \\
173 & $\mathrm{H}$ & -1.4071 & -3.3261 & 4.3065 & $\mathrm{O}$ & -5.3350 & 1.9599 & 1.3532 \\
174 & $\mathrm{H}$ & 0.1205 & -2.6970 & 4.9842 & $\mathrm{O}$ & -3.1424 & 2.0592 & 3.2834 \\
175 & $\mathrm{H}$ & -0.6096 & -6.2679 & -2.3775 & $\mathrm{H}$ & -6.8406 & 0.8866 & 0.6133 \\
176 & $\mathrm{H}$ & 6.8017 & -0.6100 & 0.2555 & $\mathrm{H}$ & -6.9379 & 3.1075 & -0.4850 \\
177 & $\mathrm{H}$ & 6.7742 & -2.8170 & -0.8460 & $\mathrm{H}$ & -4.2391 & 2.3760 & -1.6891 \\
178 & $\mathrm{H}$ & 3.9430 & -2.1201 & -1.7277 & $\mathrm{H}$ & -4.8441 & 4.4611 & 0.4430 \\
179 & $\mathrm{H}$ & 4.9325 & -4.1000 & 0.3711 & $\mathrm{H}$ & -3.3781 & 1.7908 & 0.6898 \\
180 & $\mathrm{H}$ & 3.3643 & -1.4999 & 0.6753 & $\mathrm{H}$ & -4.3734 & 3.6203 & 2.9028 \\
181 & $\mathrm{H}$ & 2.5226 & -2.9362 & 2.3395 & $\mathrm{H}$ & -2.7364 & 3.8013 & 2.2276 \\
182 & $\mathrm{H}$ & 3.8120 & -1.9957 & 3.1376 & $\mathrm{H}$ & -8.3607 & 0.9982 & -2.9158 \\
183 & $\mathrm{H}$ & 6.3909 & -2.8462 & -3.7119 & $\mathrm{H}$ & -8.1681 & 0.4929 & -1.2250 \\
184 & $\mathrm{H}$ & 7.9374 & -2.6285 & -2.8491 & $\mathrm{H}$ & -8.6955 & 2.1593 & -1.6022 \\
185 & $\mathrm{H}$ & 7.4000 & -1.3965 & -4.0208 & $\mathrm{H}$ & -1.5970 & 0.8373 & 3.7597 \\
186 & $\mathrm{H}$ & 2.6676 & -4.7166 & 3.7521 & $\mathrm{H}$ & -1.7599 & 1.1331 & 2.0134 \\
187 & $\mathrm{H}$ & 3.9321 & -3.9120 & 4.7335 & $\mathrm{H}$ & -1.1089 & 2.4107 & 3.0722 \\
188 & $\mathrm{H}$ & 4.2555 & -5.5050 & 4.0016 & $\mathrm{H}$ & -4.3774 & 4.6227 & -2.2964 \\
189 & $\mathrm{H}$ & 4.0529 & -4.4171 & -2.2598 & $\mathrm{H}$ & -0.5493 & 4.8665 & 3.0035 \\
\hline & & & & & & & & \\
& & & & & & &
\end{tabular}


Table S11. Cartesian coordinates of atoms in the DM- $\beta-C D$ structures ZULQAY in vacuo and BOYFOK03 in water, obtained after the M062X-GD3/6-31++G(d,p) re-optimization of the structures given in Table S10.

\begin{tabular}{|c|c|c|c|c|c|c|c|c|}
\hline \multicolumn{5}{|c|}{ ZULQAY } & \multicolumn{4}{|c|}{ BOYFOK03 } \\
\hline No. & Atom & $\mathbf{x}$ & $\mathbf{y}$ & $\mathbf{Z}$ & Atom & $\mathbf{x}$ & $\mathbf{y}$ & $\mathbf{Z}$ \\
\hline 1 & $\mathrm{C}$ & 4.4761 & 4.0818 & -0.3800 & $\mathrm{C}$ & -1.8188 & 5.7773 & -0.1587 \\
\hline 2 & $\mathrm{C}$ & 5.0827 & 3.5048 & -1.6624 & $\mathrm{C}$ & -1.0545 & 6.2137 & -1.4138 \\
\hline 3 & $\mathrm{C}$ & 4.8777 & 1.9969 & -1.7134 & $\mathrm{C}$ & 0.2038 & 5.3750 & -1.5921 \\
\hline 4 & $\mathrm{C}$ & 5.3766 & 1.3724 & -0.4145 & $\mathrm{C}$ & 1.0002 & 5.3893 & -0.2939 \\
\hline 5 & $\mathrm{C}$ & 4.7740 & 2.0509 & 0.8137 & $\mathrm{C}$ & 0.1376 & 4.9286 & 0.8791 \\
\hline 6 & $\mathrm{C}$ & 5.3515 & 1.5113 & 2.1151 & $\mathrm{C}$ & 0.8839 & 4.9426 & 2.1944 \\
\hline 7 & $\mathrm{C}$ & 5.2368 & 4.2232 & -3.9434 & $\mathrm{C}$ & -1.7783 & 7.0820 & -3.5223 \\
\hline 8 & $\mathrm{C}$ & 7.4920 & 2.5525 & 2.1952 & $\mathrm{C}$ & 2.4059 & 6.1602 & 3.4812 \\
\hline 9 & $\mathrm{O}$ & 4.4772 & 4.1876 & -2.7404 & $\mathrm{O}$ & -1.9415 & 6.0923 & -2.5098 \\
\hline 10 & $\mathrm{O}$ & 5.5853 & 1.4727 & -2.8130 & $\mathrm{O}$ & 0.9701 & 5.9075 & -2.6527 \\
\hline 11 & $\mathrm{O}$ & 4.9938 & 0.0096 & -0.4628 & $\mathrm{O}$ & 2.1110 & 4.5290 & -0.4868 \\
\hline 12 & $\mathrm{O}$ & 5.0199 & 3.4599 & 0.7577 & $\mathrm{O}$ & -0.9877 & 5.8071 & 0.9763 \\
\hline 13 & $\mathrm{O}$ & 6.7513 & 1.3505 & 2.1053 & $\mathrm{O}$ & 1.5654 & 6.1687 & 2.3412 \\
\hline 14 & $\mathrm{C}$ & -0.4040 & 5.9746 & 0.3146 & $\mathrm{H}$ & -2.6346 & 6.4780 & 0.0448 \\
\hline 15 & $\mathrm{C}$ & 0.4723 & 6.5269 & -0.8146 & $\mathrm{H}$ & -0.7645 & 7.2661 & -1.2817 \\
\hline 16 & $\mathrm{C}$ & 1.5092 & 5.4915 & -1.2261 & $\mathrm{H}$ & -0.0922 & 4.3376 & -1.8127 \\
\hline 17 & $\mathrm{C}$ & 2.2601 & 4.9763 & 0.0012 & $\mathrm{H}$ & 1.3460 & 6.4121 & -0.0961 \\
\hline 18 & $\mathrm{C}$ & 1.2867 & 4.4872 & 1.0715 & $\mathrm{H}$ & -0.2130 & 3.9012 & 0.6953 \\
\hline 19 & $\mathrm{C}$ & 1.9789 & 4.0952 & 2.3561 & $\mathrm{H}$ & 1.6044 & 4.1130 & 2.2081 \\
\hline 20 & $\mathrm{C}$ & 0.0510 & 7.9276 & -2.7083 & $\mathrm{H}$ & -2.5409 & 6.8841 & -4.2762 \\
\hline 21 & $\mathrm{C}$ & 1.6115 & 3.0146 & 4.3997 & $\mathrm{H}$ & -1.9315 & 8.0834 & -3.1042 \\
\hline 22 & $\mathrm{O}$ & -0.3999 & 6.8722 & -1.8701 & $\mathrm{H}$ & -0.7840 & 7.0154 & -3.9697 \\
\hline 23 & $\mathrm{O}$ & 2.4084 & 6.0744 & -2.1409 & $\mathrm{H}$ & 2.9194 & 7.1222 & 3.5135 \\
\hline 24 & $\mathrm{O}$ & 3.0877 & 3.9093 & -0.4376 & $\mathrm{H}$ & 1.8155 & 6.0347 & 4.3990 \\
\hline 25 & $\mathrm{O}$ & 0.3877 & 5.5459 & 1.3930 & $\mathrm{H}$ & 3.1381 & 5.3473 & 3.4058 \\
\hline 26 & $\mathrm{O}$ & 1.0560 & 3.4017 & 3.1655 & $\mathrm{H}$ & 1.8738 & 5.5444 & -2.5916 \\
\hline 27 & $\mathrm{C}$ & -5.0253 & 3.4999 & 0.1248 & $\mathrm{C}$ & 3.3775 & 4.9864 & -0.0863 \\
\hline 28 & $\mathrm{C}$ & -4.7400 & 4.4450 & -1.0479 & $\mathrm{C}$ & 4.3519 & 4.7167 & -1.2388 \\
\hline 29 & $\mathrm{C}$ & -3.2393 & 4.5736 & -1.2808 & $\mathrm{C}$ & 4.5499 & 3.2188 & -1.4106 \\
\hline 30 & $\mathrm{C}$ & -2.5589 & 4.9110 & 0.0407 & $\mathrm{C}$ & 4.9347 & 2.5819 & -0.0776 \\
\hline 31 & $\mathrm{C}$ & -2.9202 & 3.8970 & 1.1227 & $\mathrm{C}$ & 3.8983 & 2.9301 & 0.9916 \\
\hline 32 & $\mathrm{C}$ & -2.2701 & 4.2025 & 2.4550 & $\mathrm{C}$ & 4.2749 & 2.4145 & 2.3691 \\
\hline 33 & C & -5.7809 & 4.8803 & -3.1604 & C & 4.7611 & 5.8626 & -3.2983 \\
\hline 34 & $\mathrm{C}$ & -1.6433 & 5.9263 & 3.8920 & $\mathrm{C}$ & 2.2500 & 1.5304 & 3.2132 \\
\hline 35 & $\mathrm{O}$ & -5.4354 & 3.9271 & -2.1629 & $\mathrm{O}$ & 3.8035 & 5.3159 & -2.3970 \\
\hline 36 & $\mathrm{O}$ & -3.0073 & 5.5909 & -2.2284 & $\mathrm{O}$ & 5.5467 & 2.9802 & -2.3810 \\
\hline 37 & $\mathrm{O}$ & -1.1634 & 4.9215 & -0.2142 & $\mathrm{O}$ & 4.9806 & 1.1836 & -0.3170 \\
\hline 38 & $\mathrm{O}$ & -4.3410 & 3.9060 & 1.2817 & $\mathrm{O}$ & 3.8054 & 4.3515 & 1.0907 \\
\hline 39 & $\mathrm{O}$ & -2.3622 & 5.5823 & 2.7276 & $\mathrm{O}$ & 3.2243 & 2.5517 & 3.3062 \\
\hline 40 & $\mathrm{C}$ & -5.8822 & -1.7234 & 0.0253 & $\mathrm{H}$ & 3.3429 & 6.0549 & 0.1453 \\
\hline 41 & $\mathrm{C}$ & -6.5550 & -0.8791 & -1.0624 & $\mathrm{H}$ & 5.3149 & 5.1862 & -0.9908 \\
\hline 42 & $\mathrm{C}$ & -5.7840 & 0.4150 & -1.2884 & $\mathrm{H}$ & 3.5922 & 2.7785 & -1.7290 \\
\hline 43 & $\mathrm{C}$ & -5.5033 & 1.1164 & 0.0408 & $\mathrm{H}$ & 5.9253 & 2.9493 & 0.2312 \\
\hline 44 & $\mathrm{C}$ & -4.8410 & 0.1548 & 1.0254 & $\mathrm{H}$ & 2.9269 & 2.5172 & 0.6825 \\
\hline 45 & $\mathrm{C}$ & -4.6195 & 0.7573 & 2.4010 & $\mathrm{H}$ & 5.1147 & 3.0074 & 2.7467 \\
\hline 46 & $\mathrm{C}$ & -7.6633 & -1.3861 & -3.1220 & $\mathrm{H}$ & 4.5912 & 1.3652 & 2.2946 \\
\hline 47 & $\mathrm{C}$ & -2.4319 & 0.0294 & 2.9265 & $\mathrm{H}$ & 4.1976 & 6.3061 & -4.1199 \\
\hline 48 & $\mathrm{O}$ & -6.6112 & -1.6912 & -2.2168 & $\mathrm{H}$ & 5.3510 & 6.6411 & -2.8010 \\
\hline
\end{tabular}




\begin{tabular}{|c|c|c|c|c|c|c|c|c|}
\hline 49 & $\mathrm{O}$ & -6.5297 & 1.2554 & -2.1378 & $\mathrm{H}$ & 5.4244 & 5.0822 & -3.6792 \\
\hline 50 & $\mathrm{O}$ & -4.6485 & 2.2069 & -0.2663 & $\mathrm{H}$ & 1.5310 & 1.6974 & 4.0171 \\
\hline 51 & $\mathrm{O}$ & -5.6979 & -0.9720 & 1.1977 & $\mathrm{H}$ & 1.7185 & 1.5509 & 2.2515 \\
\hline 52 & $\mathrm{O}$ & -3.8077 & -0.0529 & 3.2275 & $\mathrm{H}$ & 2.7090 & 0.5405 & 3.3393 \\
\hline 53 & $\mathrm{C}$ & -2.3864 & -5.6145 & -0.2897 & $\mathrm{H}$ & 5.7162 & 2.0196 & -2.4025 \\
\hline 54 & $\mathrm{C}$ & -3.2395 & -5.4227 & -1.5491 & $\mathrm{C}$ & 6.1181 & 0.4557 & 0.0650 \\
\hline 55 & $\mathrm{C}$ & -3.7091 & -3.9770 & -1.6627 & $\mathrm{C}$ & 6.5077 & -0.4469 & -1.1173 \\
\hline 56 & $\mathrm{C}$ & -4.3414 & -3.5519 & -0.3427 & $\mathrm{C}$ & 5.4222 & -1.4815 & -1.3653 \\
\hline 57 & $\mathrm{C}$ & -3.3932 & -3.7941 & 0.8283 & $\mathrm{C}$ & 5.1133 & -2.1998 & -0.0567 \\
\hline 58 & $\mathrm{C}$ & -3.9829 & -3.3952 & 2.1643 & $\mathrm{C}$ & 4.7667 & -1.2150 & 1.0584 \\
\hline 59 & $\mathrm{C}$ & -3.1731 & -6.2635 & -3.7889 & $\mathrm{C}$ & 4.5030 & -1.8981 & 2.3820 \\
\hline 60 & $\mathrm{C}$ & -5.9443 & -3.3435 & 3.4203 & $\mathrm{C}$ & 8.0299 & 0.8068 & -2.4457 \\
\hline 61 & $\mathrm{O}$ & -2.4475 & -5.8441 & -2.6397 & $\mathrm{C}$ & 5.2041 & -3.6264 & 3.7852 \\
\hline 62 & $\mathrm{O}$ & -4.6495 & -3.8728 & -2.7082 & $\mathrm{O}$ & 6.6990 & 0.3255 & -2.2871 \\
\hline 63 & $\mathrm{O}$ & -4.6560 & -2.1762 & -0.4836 & $\mathrm{O}$ & 5.8751 & -2.4022 & -2.3344 \\
\hline 64 & $\mathrm{O}$ & -3.0703 & -5.1863 & 0.8602 & $\mathrm{O}$ & 4.0329 & -3.0779 & -0.3244 \\
\hline 65 & $\mathrm{O}$ & -5.3216 & -3.8247 & 2.2478 & $\mathrm{O}$ & 5.8678 & -0.3144 & 1.2155 \\
\hline 66 & $\mathrm{C}$ & 2.8516 & -5.2237 & 0.2405 & $\mathrm{O}$ & 5.5098 & -2.8523 & 2.6394 \\
\hline 67 & $\mathrm{C}$ & 2.2897 & -6.1051 & -0.8868 & $\mathrm{H}$ & 6.9381 & 1.1312 & 0.3304 \\
\hline 68 & $\mathrm{C}$ & 0.8989 & -5.6371 & -1.2773 & $\mathrm{H}$ & 7.4379 & -0.9737 & -0.8598 \\
\hline 69 & $\mathrm{C}$ & 0.0207 & -5.4965 & -0.0333 & $\mathrm{H}$ & 4.5117 & -0.9689 & -1.7122 \\
\hline 70 & $\mathrm{C}$ & 0.6942 & -4.6383 & 1.0360 & $\mathrm{H}$ & 5.9983 & -2.7742 & 0.2462 \\
\hline 71 & $\mathrm{C}$ & -0.0781 & -4.6056 & 2.3344 & $\mathrm{H}$ & 3.8659 & -0.6462 & 0.7833 \\
\hline 72 & $\mathrm{C}$ & 4.1524 & -7.0304 & -2.0287 & $\mathrm{H}$ & 3.5238 & -2.3947 & 2.3440 \\
\hline 73 & $\mathrm{C}$ & -0.1410 & -3.5471 & 4.4212 & $\mathrm{H}$ & 4.4797 & -1.1391 & 3.1804 \\
\hline 74 & $\mathrm{O}$ & 3.1367 & -6.0442 & -2.0161 & $\mathrm{H}$ & 8.0499 & 1.3799 & -3.3731 \\
\hline 75 & $\mathrm{O}$ & 0.3340 & -6.5722 & -2.1606 & $\mathrm{H}$ & 8.3217 & 1.4597 & -1.6148 \\
\hline 76 & $\mathrm{O}$ & -1.1924 & -4.9018 & -0.4655 & $\mathrm{H}$ & 8.7322 & -0.0304 & -2.5165 \\
\hline 77 & $\mathrm{O}$ & 1.9722 & -5.1961 & 1.3350 & $\mathrm{H}$ & 6.0001 & -4.3631 & 3.9025 \\
\hline 78 & $\mathrm{O}$ & 0.4588 & -3.5853 & 3.1482 & $\mathrm{H}$ & 5.1617 & -2.9940 & 4.6824 \\
\hline 79 & $\mathrm{C}$ & 5.9065 & -0.9701 & -0.0238 & $\mathrm{H}$ & 4.2409 & -4.1341 & 3.6530 \\
\hline 80 & $\mathrm{C}$ & 6.0687 & -1.9820 & -1.1663 & $\mathrm{H}$ & 5.3050 & -3.1930 & -2.2945 \\
\hline 81 & $\mathrm{C}$ & 4.7683 & -2.7458 & -1.3819 & $\mathrm{C}$ & 4.1200 & -4.3953 & 0.1564 \\
\hline 82 & $\mathrm{C}$ & 4.2999 & -3.3105 & -0.0478 & $\mathrm{C}$ & 3.7409 & -5.3332 & -0.9941 \\
\hline 83 & $\mathrm{C}$ & 4.1742 & -2.2128 & 1.0037 & $\mathrm{C}$ & 2.2656 & -5.1809 & -1.3327 \\
\hline 84 & $\mathrm{C}$ & 3.7280 & -2.7468 & 2.3478 & $\mathrm{C}$ & 1.4140 & -5.3072 & -0.0710 \\
\hline 85 & $\mathrm{C}$ & 7.2010 & -1.9671 & -3.2755 & $\mathrm{C}$ & 1.8988 & -4.3334 & 1.0031 \\
\hline 86 & C & 3.9357 & -4.5403 & 3.8213 & $\mathrm{C}$ & 1.1609 & -4.4992 & 2.3189 \\
\hline 87 & $\mathrm{O}$ & 6.4681 & -1.2376 & -2.2998 & $\mathrm{C}$ & 4.9743 & -6.1131 & -2.8892 \\
\hline 88 & $\mathrm{O}$ & 4.9814 & -3.7913 & -2.3054 & $\mathrm{C}$ & 0.7970 & -2.2591 & 2.9987 \\
\hline 89 & $\mathrm{O}$ & 3.0535 & -3.9417 & -0.2882 & $\mathrm{O}$ & 4.5726 & -5.0046 & -2.0910 \\
\hline 90 & $\mathrm{O}$ & 5.4507 & -1.5879 & 1.1483 & $\mathrm{O}$ & 1.9035 & -6.1614 & -2.2815 \\
\hline 91 & $\mathrm{O}$ & 4.4313 & -3.9313 & 2.6491 & $\mathrm{O}$ & 0.0782 & -5.0112 & -0.4571 \\
\hline 92 & $\mathrm{H}$ & 4.7292 & 5.1442 & -0.2943 & $\mathrm{O}$ & 3.2794 & -4.5947 & 1.2621 \\
\hline 93 & $\mathrm{H}$ & 6.1646 & 3.7139 & -1.6495 & $\mathrm{O}$ & 1.4703 & -3.4806 & 3.2527 \\
\hline 94 & $\mathrm{H}$ & 3.7994 & 1.7957 & -1.8066 & $\mathrm{H}$ & 5.1341 & -4.6082 & 0.5071 \\
\hline 95 & $\mathrm{H}$ & 6.4723 & 1.4574 & -0.3804 & $\mathrm{H}$ & 3.9339 & -6.3663 & -0.6704 \\
\hline 96 & $\mathrm{H}$ & 3.6881 & 1.8685 & 0.8131 & $\mathrm{H}$ & 2.1083 & -4.1712 & -1.7428 \\
\hline 97 & $\mathrm{H}$ & 4.9434 & 0.5113 & 2.2856 & $\mathrm{H}$ & 1.4794 & -6.3361 & 0.3134 \\
\hline 98 & $\mathrm{H}$ & 5.0436 & 2.1765 & 2.9376 & $\mathrm{H}$ & 1.7828 & -3.3075 & 0.6250 \\
\hline 99 & $\mathrm{H}$ & 5.3471 & 3.2225 & -4.3648 & $\mathrm{H}$ & 1.4732 & -5.4456 & 2.7745 \\
\hline 100 & $\mathrm{H}$ & 6.2286 & 4.6529 & -3.7536 & $\mathrm{H}$ & 0.0794 & -4.5356 & 2.1421 \\
\hline 101 & $\mathrm{H}$ & 4.6901 & 4.8694 & -4.6317 & $\mathrm{H}$ & 5.6156 & -5.7148 & -3.6762 \\
\hline 102 & $\mathrm{H}$ & 7.1091 & 3.1863 & 3.0065 & $\mathrm{H}$ & 5.5430 & -6.8307 & -2.2865 \\
\hline 103 & $\mathrm{H}$ & 7.4549 & 3.1267 & 1.2626 & $\mathrm{H}$ & 4.1054 & -6.6075 & -3.3304 \\
\hline 104 & $\mathrm{H}$ & 8.5232 & 2.2695 & 2.4129 & $\mathrm{H}$ & 0.9922 & -1.6047 & 3.8507 \\
\hline
\end{tabular}




\begin{tabular}{|c|c|c|c|c|c|c|c|c|}
\hline 105 & $\mathrm{H}$ & 5.6936 & 0.5122 & -2.6841 & $\mathrm{H}$ & 1.1690 & -1.7671 & 2.0888 \\
\hline 106 & $\mathrm{H}$ & -1.0570 & 6.7545 & 0.7168 & $\mathrm{H}$ & -0.2830 & -2.4278 & 2.9024 \\
\hline 107 & $\mathrm{H}$ & 0.9893 & 7.4258 & -0.4433 & $\mathrm{H}$ & 0.9444 & -6.0869 & -2.4476 \\
\hline 108 & $\mathrm{H}$ & 0.9821 & 4.6394 & -1.6813 & $\mathrm{C}$ & -0.9039 & -5.9946 & -0.2713 \\
\hline 109 & $\mathrm{H}$ & 2.8758 & 5.7901 & 0.4151 & C & -1.7893 & -6.0218 & -1.5211 \\
\hline 110 & $\mathrm{H}$ & 0.7273 & 3.6222 & 0.6872 & $\mathrm{C}$ & -2.5885 & -4.7323 & -1.6227 \\
\hline 111 & $\mathrm{H}$ & 2.3334 & 5.0051 & 2.8683 & $\mathrm{C}$ & -3.3307 & -4.4717 & -0.3143 \\
\hline 112 & $\mathrm{H}$ & 2.8501 & 3.4638 & 2.1299 & $\mathrm{C}$ & -2.3549 & -4.4876 & 0.8641 \\
\hline 113 & $\mathrm{H}$ & 0.9751 & 7.6523 & -3.2212 & C & -3.0453 & -4.3340 & 2.1998 \\
\hline 114 & $\mathrm{H}$ & 0.2139 & 8.8385 & -2.1178 & $\mathrm{C}$ & -1.4996 & -7.0206 & -3.6741 \\
\hline 115 & $\mathrm{H}$ & -0.7460 & 8.1080 & -3.4310 & $\mathrm{C}$ & -2.6191 & -3.9496 & 4.4742 \\
\hline 116 & $\mathrm{H}$ & 1.9394 & 3.8895 & 4.9788 & $\mathrm{O}$ & -0.9449 & -6.2114 & -2.6402 \\
\hline 117 & $\mathrm{H}$ & 2.4714 & 2.3446 & 4.2539 & $\mathrm{O}$ & -3.4967 & -4.8289 & -2.6983 \\
\hline 118 & $\mathrm{H}$ & 0.8354 & 2.4863 & 4.9556 & $\mathrm{O}$ & -3.9429 & -3.2007 & -0.4685 \\
\hline 119 & $\mathrm{H}$ & 3.0419 & 5.3910 & -2.4275 & $\mathrm{O}$ & -1.6738 & -5.7440 & 0.8777 \\
\hline 120 & $\mathrm{H}$ & -6.0913 & 3.5294 & 0.3757 & $\mathrm{O}$ & -2.0593 & -4.1642 & 3.1944 \\
\hline 121 & $\mathrm{H}$ & -5.1380 & 5.4382 & -0.7874 & $\mathrm{H}$ & -0.4437 & -6.9758 & -0.1175 \\
\hline 122 & $\mathrm{H}$ & -2.8560 & 3.6062 & -1.6411 & $\mathrm{H}$ & -2.4816 & -6.8706 & -1.4247 \\
\hline 123 & $\mathrm{H}$ & -2.8898 & 5.9046 & 0.3693 & $\mathrm{H}$ & -1.8864 & -3.8999 & -1.7853 \\
\hline 124 & $\mathrm{H}$ & -2.5966 & 2.8935 & 0.8059 & $\mathrm{H}$ & -4.0966 & -5.2493 & -0.1714 \\
\hline 125 & $\mathrm{H}$ & -1.2147 & 3.8974 & 2.4232 & $\mathrm{H}$ & -1.6279 & -3.6710 & 0.7398 \\
\hline 126 & $\mathrm{H}$ & -2.7801 & 3.6206 & 3.2409 & $\mathrm{H}$ & -3.6532 & -5.2296 & 2.4066 \\
\hline 127 & $\mathrm{H}$ & -4.8876 & 5.2958 & -3.6308 & $\mathrm{H}$ & -3.7119 & -3.4608 & 2.1643 \\
\hline 128 & $\mathrm{H}$ & -6.3748 & 5.6917 & -2.7210 & $\mathrm{H}$ & -0.7412 & -7.0922 & -4.4542 \\
\hline 129 & $\mathrm{H}$ & -6.3870 & 4.3486 & -3.8955 & $\mathrm{H}$ & -1.7259 & -8.0231 & -3.2933 \\
\hline 130 & $\mathrm{H}$ & -0.5867 & 5.6471 & 3.7856 & $\mathrm{H}$ & -2.4063 & -6.5625 & -4.0760 \\
\hline 131 & $\mathrm{H}$ & -2.0670 & 5.4261 & 4.7749 & $\mathrm{H}$ & -1.7913 & -3.8109 & 5.1711 \\
\hline 132 & $\mathrm{H}$ & -1.7293 & 7.0067 & 4.0196 & $\mathrm{H}$ & -3.2529 & -3.0529 & 4.4799 \\
\hline 133 & $\mathrm{H}$ & -2.0694 & 5.8507 & -2.1809 & $\mathrm{H}$ & -3.2205 & -4.8118 & 4.7903 \\
\hline 134 & $\mathrm{H}$ & -6.5155 & -2.5693 & 0.3076 & $\mathrm{H}$ & -4.0921 & -4.0567 & -2.6605 \\
\hline 135 & $\mathrm{H}$ & -7.5739 & -0.6312 & -0.7253 & $\mathrm{C}$ & -5.2961 & -3.0407 & -0.1285 \\
\hline 136 & $\mathrm{H}$ & -4.8131 & 0.1596 & -1.7412 & $\mathrm{C}$ & -5.9942 & -2.3455 & -1.3126 \\
\hline 137 & $\mathrm{H}$ & -6.4518 & 1.4823 & 0.4644 & $\mathrm{C}$ & -5.4630 & -0.9311 & -1.4745 \\
\hline 138 & $\mathrm{H}$ & -3.8832 & -0.1720 & 0.5959 & $\mathrm{C}$ & -5.5596 & -0.2126 & -0.1347 \\
\hline 139 & $\mathrm{H}$ & -5.5875 & 0.8430 & 2.9061 & $\mathrm{C}$ & -4.8312 & -0.9895 & 0.9589 \\
\hline 140 & $\mathrm{H}$ & -4.1884 & 1.7621 & 2.2983 & C & -4.9051 & -0.3156 & 2.3100 \\
\hline 141 & $\mathrm{H}$ & -7.5429 & -0.3848 & -3.5413 & $\mathrm{C}$ & -6.7271 & -4.1013 & -2.7384 \\
\hline 142 & $\mathrm{H}$ & -8.6340 & -1.4564 & -2.6143 & $\mathrm{C}$ & -6.3142 & 0.8651 & 3.7501 \\
\hline 143 & $\mathrm{H}$ & -7.6169 & -2.1378 & -3.9113 & $\mathrm{O}$ & -5.7765 & -3.0664 & -2.5092 \\
\hline 144 & $\mathrm{H}$ & -2.0833 & 1.0725 & 2.9436 & $\mathrm{O}$ & -6.2273 & -0.2527 & -2.4484 \\
\hline 145 & $\mathrm{H}$ & -2.1924 & -0.4034 & 1.9450 & $\mathrm{O}$ & -4.9972 & 1.0729 & -0.3301 \\
\hline 146 & $\mathrm{H}$ & -1.9011 & -0.5374 & 3.6935 & $\mathrm{O}$ & -5.4364 & -2.2840 & 1.0485 \\
\hline 147 & $\mathrm{H}$ & -6.0587 & 2.1052 & -2.2201 & $\mathrm{O}$ & -6.2336 & 0.0808 & 2.5739 \\
\hline 148 & $\mathrm{H}$ & -2.1797 & -6.6801 & -0.1420 & $\mathrm{H}$ & -5.7569 & -4.0110 & 0.0842 \\
\hline 149 & $\mathrm{H}$ & -4.1259 & -6.0702 & -1.4576 & $\mathrm{H}$ & -7.0715 & -2.2916 & -1.0979 \\
\hline 150 & $\mathrm{H}$ & -2.8329 & -3.3392 & -1.8580 & $\mathrm{H}$ & -4.4054 & -0.9782 & -1.7770 \\
\hline 151 & $\mathrm{H}$ & -5.2575 & -4.1346 & -0.1807 & $\mathrm{H}$ & -6.6177 & -0.1229 & 0.1448 \\
\hline 152 & $\mathrm{H}$ & -2.4727 & -3.2106 & 0.6730 & $\mathrm{H}$ & -3.7702 & -1.1005 & 0.6933 \\
\hline 153 & $\mathrm{H}$ & -3.9378 & -2.3027 & 2.2736 & $\mathrm{H}$ & -4.2487 & 0.5655 & 2.3087 \\
\hline 154 & $\mathrm{H}$ & -3.3813 & -3.8535 & 2.9668 & $\mathrm{H}$ & -4.5477 & -1.0147 & 3.0822 \\
\hline 155 & $\mathrm{H}$ & -3.7232 & -5.4301 & -4.2302 & $\mathrm{H}$ & -6.4613 & -4.5696 & -3.6863 \\
\hline 156 & $\mathrm{H}$ & -3.8746 & -7.0651 & -3.5245 & $\mathrm{H}$ & -6.6970 & -4.8572 & -1.9449 \\
\hline 157 & $\mathrm{H}$ & -2.4349 & -6.6489 & -4.4937 & $\mathrm{H}$ & -7.7369 & -3.6830 & -2.8056 \\
\hline 158 & $\mathrm{H}$ & -5.9047 & -2.2472 & 3.4534 & $\mathrm{H}$ & -7.3567 & 1.1621 & 3.8730 \\
\hline 159 & $\mathrm{H}$ & -5.4573 & -3.7524 & 4.3171 & $\mathrm{H}$ & -5.9976 & 0.2852 & 4.6276 \\
\hline 160 & $\mathrm{H}$ & -6.9831 & -3.6768 & 3.3958 & $\mathrm{H}$ & -5.6814 & 1.7561 & 3.6555 \\
\hline
\end{tabular}




\begin{tabular}{rlrrrrrrr}
161 & $\mathrm{H}$ & -5.1393 & -3.0377 & -2.5983 & $\mathrm{H}$ & -6.0192 & 0.6975 & -2.3801 \\
162 & $\mathrm{H}$ & 3.7909 & -5.6244 & 0.6358 & $\mathrm{C}$ & -5.6770 & 2.1807 & 0.2015 \\
163 & $\mathrm{H}$ & 2.2171 & -7.1416 & -0.5235 & $\mathrm{C}$ & -5.7740 & 3.2378 & -0.9112 \\
164 & $\mathrm{H}$ & 0.9833 & -4.6450 & -1.7470 & $\mathrm{C}$ & -4.3878 & 3.7537 & -1.2562 \\
165 & $\mathrm{H}$ & -0.1726 & -6.5003 & 0.3767 & $\mathrm{C}$ & -3.6500 & 4.1893 & 0.0099 \\
166 & $\mathrm{H}$ & 0.8112 & -3.6119 & 0.6597 & $\mathrm{C}$ & -3.6567 & 3.0792 & 1.0594 \\
167 & $\mathrm{H}$ & 0.0174 & -5.5846 & 2.8328 & $\mathrm{C}$ & -3.0600 & 3.5165 & 2.3849 \\
168 & $\mathrm{H}$ & -1.1424 & -4.4235 & 2.1334 & $\mathrm{C}$ & -7.7800 & 2.7089 & -2.0744 \\
169 & $\mathrm{H}$ & 3.7120 & -8.0340 & -2.0604 & $\mathrm{C}$ & -1.6641 & 1.7063 & 2.9847 \\
170 & $\mathrm{H}$ & 4.8106 & -6.9457 & -1.1535 & $\mathrm{O}$ & -6.3575 & 2.6804 & -2.0730 \\
171 & $\mathrm{H}$ & 4.7448 & -6.8638 & -2.9292 & $\mathrm{O}$ & -4.5053 & 4.8361 & -2.1519 \\
172 & $\mathrm{H}$ & 0.0219 & -4.4889 & 4.9641 & $\mathrm{O}$ & -2.3197 & 4.4877 & -0.3900 \\
173 & $\mathrm{H}$ & -1.2233 & -3.3675 & 4.3456 & $\mathrm{O}$ & -5.0080 & 2.7013 & 1.3207 \\
174 & $\mathrm{H}$ & 0.3212 & -2.7286 & 4.9756 & $\mathrm{O}$ & -2.8458 & 2.4316 & 3.2667 \\
175 & $\mathrm{H}$ & -0.5506 & -6.2537 & -2.4147 & $\mathrm{H}$ & -6.6660 & 1.8851 & 0.5643 \\
176 & $\mathrm{H}$ & 6.8667 & -0.5113 & 0.2306 & $\mathrm{H}$ & -6.3855 & 4.0761 & -0.5462 \\
177 & $\mathrm{H}$ & 6.8592 & -2.6965 & -0.8892 & $\mathrm{H}$ & -3.8138 & 2.9325 & -1.7128 \\
178 & $\mathrm{H}$ & 4.0092 & -2.0425 & -1.7580 & $\mathrm{H}$ & -4.1359 & 5.0871 & 0.4209 \\
179 & $\mathrm{H}$ & 5.0349 & -4.0461 & 0.3044 & $\mathrm{H}$ & -3.1034 & 2.2178 & 0.6594 \\
180 & $\mathrm{H}$ & 3.4390 & -1.4660 & 0.6697 & $\mathrm{H}$ & -3.7643 & 4.1934 & 2.8797 \\
181 & $\mathrm{H}$ & 2.6492 & -2.9514 & 2.3186 & $\mathrm{H}$ & -2.1223 & 4.0590 & 2.2047 \\
182 & $\mathrm{H}$ & 3.9191 & -1.9798 & 3.1159 & $\mathrm{H}$ & -8.1029 & 2.2794 & -3.0231 \\
183 & $\mathrm{H}$ & 6.5918 & -2.7624 & -3.7105 & $\mathrm{H}$ & -8.1971 & 2.1139 & -1.2533 \\
184 & $\mathrm{H}$ & 8.1035 & -2.4002 & -2.8256 & $\mathrm{H}$ & -8.1422 & 3.7398 & -1.9966 \\
185 & $\mathrm{H}$ & 7.4925 & -1.2474 & -4.0420 & $\mathrm{H}$ & -1.5548 & 0.9489 & 3.7627 \\
186 & $\mathrm{H}$ & 2.8673 & -4.7680 & 3.7120 & $\mathrm{H}$ & -1.7079 & 1.2059 & 2.0081 \\
187 & $\mathrm{H}$ & 4.0836 & -3.8866 & 4.6935 & $\mathrm{H}$ & -0.7893 & 2.3711 & 3.0007 \\
188 & $\mathrm{H}$ & 4.4980 & -5.4638 & 3.9705 & $\mathrm{H}$ & -3.6107 & 5.1870 & -2.3226 \\
189 & $\mathrm{H}$ & 4.1864 & -4.3555 & -2.3075 & $\mathrm{H}$ & 0.1681 & 4.7975 & 3.0199 \\
\hline & & & & & & & &
\end{tabular}


Table S12. Cartesian coordinates of atoms in 11 different structures of MIA:DM- $\beta-C D$ in water (PCM) obtained from the B3LYP-GD2/6-31G(d,p) calculations performed using in the initial model of the complex the structure $\mathrm{W} 1$ of DM- $\beta-\mathrm{CD}$. The true minima are confirmed by zero imaginary frequencies.

\begin{tabular}{|c|c|c|c|c|c|c|c|c|c|}
\hline \multirow[b]{2}{*}{ atom } & \multicolumn{3}{|c|}{ CR1 } & \multicolumn{3}{|c|}{ NR1 } & \multicolumn{3}{|c|}{ M1 } \\
\hline & $\mathbf{x}$ & $\mathbf{y}$ & $\mathbf{z}$ & $\mathbf{x}$ & $\mathbf{y}$ & $\mathbf{z}$ & $\mathbf{x}$ & $\mathbf{y}$ & $\mathbf{Z}$ \\
\hline $\mathrm{O}$ & -4.1769 & -4.0470 & -1.7153 & 4.0540 & 3.3396 & 2.4445 & -2.6029 & 4.7575 & -2.0744 \\
\hline $\mathrm{C}$ & -2.7845 & -4.0584 & -1.3390 & 4.1338 & 1.9209 & 2.2122 & -3.1261 & 3.4194 & -1.9572 \\
\hline $\mathrm{C}$ & -2.5942 & -4.9756 & -0.1271 & 5.3975 & 1.6160 & 1.4016 & -4.2085 & 3.4085 & -0.8700 \\
\hline $\mathrm{C}$ & -3.5638 & -4.6914 & 1.0246 & 5.4567 & 2.4546 & 0.1155 & -3.6704 & 3.9538 & 0.4598 \\
\hline $\mathrm{C}$ & -4.9924 & -4.6365 & 0.4979 & 5.1935 & 3.9324 & 0.3869 & -3.0435 & 5.3239 & 0.2300 \\
\hline $\mathrm{C}$ & -5.0589 & -3.6614 & -0.6877 & 3.9454 & 4.0965 & 1.2649 & -2.0040 & 5.2387 & -0.9037 \\
\hline $\mathrm{H}$ & -2.4640 & -3.0423 & -1.0801 & 3.2639 & 1.5968 & 1.6297 & -2.3230 & 2.7379 & -1.6608 \\
\hline $\mathrm{H}$ & -2.7443 & -6.0149 & -0.4506 & 6.2941 & 1.8027 & 2.0075 & -5.0586 & 4.0262 & -1.1907 \\
\hline $\mathrm{H}$ & -3.3179 & -3.7028 & 1.4359 & 4.6385 & 2.1019 & -0.5218 & -2.8717 & 3.2841 & 0.8046 \\
\hline $\mathrm{H}$ & -5.2956 & -5.6337 & 0.1493 & 6.0537 & 4.3705 & 0.9116 & -3.8196 & 6.0350 & -0.0840 \\
\hline $\mathrm{H}$ & -6.0609 & -3.6748 & -1.1270 & 3.8431 & 5.1395 & 1.5784 & -1.6329 & 6.2442 & -1.1284 \\
\hline $\mathrm{C}$ & -2.0044 & -4.5918 & -2.5379 & 4.1305 & 1.2402 & 3.5736 & -3.6642 & 3.0268 & -3.3263 \\
\hline $\mathrm{O}$ & -1.2719 & -4.7973 & 0.3834 & 5.3496 & 0.2533 & 0.9746 & -4.6383 & 2.0771 & -0.5955 \\
\hline $\mathrm{O}$ & -3.4472 & -5.7015 & 2.0119 & 6.7077 & 2.3137 & -0.5272 & -4.7007 & 4.0533 & 1.4246 \\
\hline $\mathrm{O}$ & -5.9249 & -4.1534 & 1.4569 & 4.9238 & 4.6651 & -0.8046 & -2.3785 & 5.8266 & 1.3881 \\
\hline $\mathrm{O}$ & -4.7391 & -2.3722 & -0.2058 & 2.8129 & 3.6858 & 0.5195 & -0.9242 & 4.3764 & -0.5718 \\
\hline $\mathrm{H}$ & -1.0175 & -4.9395 & -2.2055 & 4.4178 & 0.1863 & 3.4442 & -4.1842 & 2.0625 & -3.2426 \\
\hline $\mathrm{O}$ & -1.8741 & -3.6528 & -3.5983 & 2.8810 & 1.3469 & 4.2419 & -2.6552 & 2.9742 & -4.3261 \\
\hline $\mathrm{H}$ & -2.5576 & -5.4494 & -2.9381 & 4.8743 & 1.7335 & 4.2098 & -4.3811 & 3.7899 & -3.6504 \\
\hline $\mathrm{H}$ & -2.5532 & -5.6120 & 2.4052 & 6.8611 & 1.3573 & -0.6779 & -5.2335 & 3.2309 & 1.3924 \\
\hline $\mathrm{C}$ & -6.4283 & -5.1328 & 2.3684 & 6.0728 & 5.1068 & -1.5314 & -3.2102 & 6.5599 & 2.2966 \\
\hline $\mathrm{C}$ & -0.7912 & -2.7535 & -3.3819 & 1.9144 & 0.4322 & 3.7293 & -1.8161 & 1.8300 & -4.2133 \\
\hline $\mathrm{O}$ & -6.2104 & 0.7391 & -1.4600 & -0.2453 & 5.6961 & 0.6850 & 2.4612 & 5.6291 & 0.0613 \\
\hline $\mathrm{C}$ & -5.2989 & -0.3743 & -1.4551 & 0.6579 & 4.6649 & 1.1254 & 1.4130 & 5.0086 & -0.7120 \\
\hline $\mathrm{C}$ & -5.7057 & -1.3297 & -0.3332 & 1.8517 & 4.6823 & 0.1735 & 0.1564 & 4.9465 & 0.1642 \\
\hline $\mathrm{C}$ & -5.7380 & -0.6161 & 1.0268 & 1.3995 & 4.3550 & -1.2523 & 0.4463 & 4.0567 & 1.3802 \\
\hline $\mathrm{C}$ & -6.5525 & 0.6770 & 0.9398 & 0.1939 & 5.2095 & -1.6667 & 1.6391 & 4.6539 & 2.1465 \\
\hline $\mathrm{C}$ & -6.1288 & 1.5089 & -0.2793 & -0.8566 & 5.3876 & -0.5491 & 2.8288 & 4.8584 & 1.1901 \\
\hline $\mathrm{H}$ & -4.2842 & -0.0182 & -1.2454 & 0.1676 & 3.6868 & 1.0556 & 1.7148 & 3.9845 & -0.9613 \\
\hline $\mathrm{H}$ & -6.7010 & -1.7464 & -0.5366 & 2.3117 & 5.6786 & 0.1818 & -0.0991 & 5.9585 & 0.4977 \\
\hline $\mathrm{H}$ & -4.6999 & -0.3430 & 1.2736 & 1.1036 & 3.2937 & -1.2670 & 0.7663 & 3.0816 & 0.9989 \\
\hline $\mathrm{H}$ & -7.6135 & 0.4167 & 0.8224 & 0.5754 & 6.2117 & -1.9079 & 1.3559 & 5.6295 & 2.5661 \\
\hline $\mathrm{H}$ & -6.8150 & 2.3504 & -0.4148 & -1.4881 & 6.2478 & -0.7913 & 3.6303 & 5.4128 & 1.6878 \\
\hline $\mathrm{C}$ & -5.3374 & -1.0001 & -2.8398 & 1.0140 & 4.9435 & 2.5771 & 1.2541 & 5.7832 & -2.0290 \\
\hline $\mathrm{O}$ & -6.3033 & -1.4481 & 2.0225 & 2.4495 & 4.5961 & -2.1803 & -0.6829 & 3.8358 & 2.2030 \\
\hline $\mathrm{O}$ & -6.3798 & 1.5178 & 2.0754 & -0.4919 & 4.6687 & -2.7925 & 2.1043 & 3.7914 & 3.1808 \\
\hline $\mathrm{O}$ & -4.8170 & 1.9603 & -0.0524 & -1.6298 & 4.2208 & -0.4467 & 3.2608 & 3.5796 & 0.8049 \\
\hline $\mathrm{H}$ & -4.8693 & -1.9929 & -2.8010 & 1.8900 & 4.3434 & 2.8553 & 0.2426 & 6.2027 & -2.1184 \\
\hline $\mathrm{O}$ & -4.6925 & -0.1851 & -3.8122 & -0.0668 & 4.6669 & 3.4597 & 1.5427 & 4.9838 & -3.1671 \\
\hline $\mathrm{H}$ & -6.3813 & -1.1071 & -3.1542 & 1.2538 & 6.0064 & 2.6895 & 1.9730 & 6.6072 & -2.0377 \\
\hline $\mathrm{H}$ & -5.9637 & -2.3568 & 1.8900 & 3.3012 & 4.4194 & -1.7319 & -1.3064 & 4.5802 & 2.0713 \\
\hline $\mathrm{C}$ & -7.2061 & 1.2102 & 3.2002 & 0.1064 & 4.9428 & -4.0625 & 1.4387 & 3.9322 & 4.4385 \\
\hline $\mathrm{C}$ & -3.2744 & -0.3050 & -3.7709 & -0.2190 & 3.2757 & 3.7231 & 0.5491 & 3.9914 & -3.4118 \\
\hline $\mathrm{O}$ & -3.1157 & 4.7698 & -1.6858 & -4.9865 & 3.7778 & 0.9664 & 6.2920 & 2.2099 & -0.6958 \\
\hline $\mathrm{C}$ & -3.5858 & 3.4138 & -1.5704 & -3.5471 & 3.8371 & 0.9979 & 5.0054 & 2.8516 & -0.6654 \\
\hline $\mathrm{C}$ & -4.4916 & 3.3195 & -0.3423 & -3.0505 & 4.3567 & -0.3529 & 4.6592 & 3.2913 & 0.7599 \\
\hline
\end{tabular}




\begin{tabular}{|c|c|c|c|c|c|c|c|c|c|}
\hline C & -3.8457 & 3.8485 & 0.9426 & -3.6122 & 3.5826 & -1.5512 & 4.8794 & 2.1903 & 1.8026 \\
\hline $\mathrm{C}$ & -3.1761 & 5.2005 & 0.7145 & -5.1213 & 3.3751 & -1.4309 & 6.2280 & 1.5048 & 1.6254 \\
\hline C & -2.3529 & 5.2027 & -0.5860 & -5.4870 & 2.8979 & -0.0136 & 6.3588 & 1.0816 & 0.1534 \\
\hline $\mathrm{H}$ & -2.7349 & 2.7319 & -1.4543 & -3.1377 & 2.8367 & 1.1790 & 4.2369 & 2.1464 & -0.9950 \\
\hline $\mathrm{H}$ & -5.4044 & 3.9007 & -0.5311 & -3.3439 & 5.4100 & -0.4562 & 5.2481 & 4.1749 & 1.0400 \\
\hline $\mathrm{H}$ & -3.0731 & 3.1434 & 1.2649 & -3.1410 & 2.5984 & -1.5555 & 4.1254 & 1.4188 & 1.6216 \\
\hline $\mathrm{H}$ & -3.9628 & 5.9634 & 0.6123 & -5.6303 & 4.3330 & -1.6126 & 7.0522 & 2.1887 & 1.8678 \\
\hline $\mathrm{H}$ & -2.0572 & 6.2303 & -0.8168 & -6.5747 & 2.8962 & 0.1049 & 7.3308 & 0.6136 & -0.0274 \\
\hline $\mathrm{C}$ & -4.3302 & 3.0837 & -2.8474 & -3.1591 & 4.7551 & 2.1371 & 5.0395 & 4.0270 & -1.6186 \\
\hline $\mathrm{O}$ & -4.8550 & 3.9812 & 1.9331 & -3.3094 & 4.2869 & -2.7463 & 4.7441 & 2.7273 & 3.1089 \\
\hline $\mathrm{O}$ & -2.2682 & 5.5830 & 1.7386 & -5.6305 & 2.3808 & -2.3140 & 6.3210 & 0.3177 & 2.4098 \\
\hline $\mathrm{O}$ & -1.2080 & 4.3919 & -0.4033 & -4.9640 & 1.6027 & 0.1570 & 5.3065 & 0.1865 & -0.1092 \\
\hline $\mathrm{H}$ & -4.8643 & 2.1338 & -2.7317 & -2.0880 & 4.9819 & 2.0834 & 4.1112 & 4.6005 & -1.4903 \\
\hline $\mathrm{O}$ & -3.4025 & 2.9998 & -3.9178 & -3.4709 & 4.1118 & 3.3641 & 5.1600 & 3.5451 & -2.9460 \\
\hline $\mathrm{H}$ & -5.0687 & 3.8807 & -3.0387 & -3.7240 & 5.6979 & 2.0425 & 5.8870 & 4.6863 & -1.3662 \\
\hline $\mathrm{H}$ & -5.2739 & 3.1049 & 2.0320 & -2.3338 & 4.3665 & -2.7815 & 3.8287 & 3.0709 & 3.1754 \\
\hline $\mathrm{C}$ & -2.8399 & 5.8146 & 3.0282 & -5.6773 & 2.7371 & -3.6967 & 6.8443 & 0.5119 & 3.7255 \\
\hline C & -4.0600 & 2.7878 & -5.1585 & -3.2098 & 4.9495 & 4.4813 & 4.9369 & 4.5768 & -3.9002 \\
\hline $\mathrm{O}$ & 1.8747 & 4.7335 & -2.3816 & -5.6307 & -1.2056 & 2.3995 & 4.6021 & -2.3498 & -2.6335 \\
\hline C & 0.6315 & 4.1081 & -2.0072 & -5.0616 & 0.0512 & 1.9908 & 4.5030 & -1.0670 & -1.9784 \\
\hline C & 0.0424 & 4.9199 & -0.8497 & -5.7870 & 0.5961 & 0.7541 & 5.5628 & -0.9821 & -0.8808 \\
\hline C & 0.9996 & 4.9251 & 0.3504 & -6.0435 & -0.4506 & -0.3415 & 5.4898 & -2.1666 & 0.0877 \\
\hline C & 2.3427 & 5.4860 & -0.1218 & -6.6036 & -1.7276 & 0.2775 & 5.5105 & -3.4734 & -0.6959 \\
\hline C & 2.8421 & 4.7149 & -1.3539 & -5.6347 & -2.1747 & 1.3870 & 4.4131 & -3.4466 & -1.7818 \\
\hline $\mathrm{H}$ & 0.8200 & 3.0782 & -1.6807 & -4.0126 & -0.0945 & 1.7264 & 3.5171 & -0.9653 & -1.5106 \\
\hline $\mathrm{H}$ & -0.0880 & 5.9555 & -1.1913 & -6.7557 & 1.0204 & 1.0519 & 6.5650 & -0.9425 & -1.3288 \\
\hline $\mathrm{H}$ & 1.1483 & 3.8904 & 0.6898 & -5.0843 & -0.7115 & -0.7973 & 4.5298 & -2.1140 & 0.6185 \\
\hline $\mathrm{H}$ & 2.1894 & 6.5313 & -0.4259 & -7.5804 & -1.5361 & 0.7423 & 6.4756 & -3.5831 & -1.2092 \\
\hline $\mathrm{H}$ & 3.7260 & 5.2132 & -1.7636 & -5.9795 & -3.1137 & 1.8336 & 4.4947 & -4.3465 & -2.4002 \\
\hline C & -0.2512 & 4.0837 & -3.2366 & -5.1712 & 0.9977 & 3.1786 & 4.6645 & 0.0009 & -3.0553 \\
\hline $\mathrm{O}$ & 0.4976 & 5.7382 & 1.3959 & -6.9313 & 0.0826 & -1.3119 & 6.5779 & -2.1180 & 0.9960 \\
\hline $\mathrm{O}$ & 3.3676 & 5.4166 & 0.8578 & -6.7256 & -2.8109 & -0.6433 & 5.2778 & -4.6165 & 0.1267 \\
\hline $\mathrm{O}$ & 3.1440 & 3.3993 & -0.9609 & -4.3035 & -2.3071 & 0.9082 & 3.1096 & -3.3335 & -1.2338 \\
\hline $\mathrm{H}$ & -1.2304 & 3.6717 & -2.9739 & -4.8921 & 2.0107 & 2.8575 & 5.0710 & 0.9199 & -2.6174 \\
\hline $\mathrm{O}$ & 0.3943 & 3.2890 & -4.2204 & -4.3952 & 0.5810 & 4.2937 & 3.4389 & 0.2564 & -3.7339 \\
\hline $\mathrm{H}$ & -0.4066 & 5.1098 & -3.6098 & -6.2159 & 1.0082 & 3.5118 & 5.3795 & -0.3694 & -3.7991 \\
\hline $\mathrm{H}$ & -0.4542 & 5.5349 & 1.5134 & -6.4741 & 0.8491 & -1.7145 & 6.4577 & -1.3126 & 1.5403 \\
\hline C & 3.4278 & 6.5271 & 1.7516 & -7.9270 & -2.8025 & -1.4261 & 6.4497 & -5.1648 & 0.7437 \\
\hline $\mathrm{C}$ & -0.4774 & 2.9004 & -5.2717 & -3.0107 & 0.8963 & 4.1641 & 2.6727 & 1.2879 & -3.1145 \\
\hline $\mathrm{O}$ & 5.3227 & 0.8734 & -2.4922 & -2.2873 & -5.3361 & 0.5177 & 0.4980 & -5.9062 & -1.1117 \\
\hline C & 4.0813 & 1.5684 & -2.2468 & -2.7778 & -4.1388 & 1.1593 & 1.2858 & -4.8419 & -1.6818 \\
\hline C & 4.3710 & 2.8193 & -1.4100 & -3.8904 & -3.5289 & 0.2953 & 2.4569 & -4.5084 & -0.7465 \\
\hline C & 5.1789 & 2.5232 & -0.1416 & -3.3738 & -3.2245 & -1.1178 & 1.9689 & -4.1898 & 0.6720 \\
\hline C & 6.3989 & 1.6678 & -0.4627 & -2.7367 & -4.4891 & -1.7089 & 1.0386 & -5.2992 & 1.1709 \\
\hline C & 5.9796 & 0.4616 & -1.3174 & -1.7143 & -5.1172 & -0.7521 & -0.0652 & -5.5850 & 0.1420 \\
\hline $\mathrm{H}$ & 3.4009 & 0.9176 & -1.6868 & -1.9640 & -3.4121 & 1.2490 & 0.6649 & -3.9453 & -1.7859 \\
\hline $\mathrm{H}$ & 4.9341 & 3.5366 & -2.0227 & -4.7317 & -4.2311 & 0.2336 & 3.1507 & -5.3557 & -0.7158 \\
\hline $\mathrm{H}$ & 4.5379 & 1.9502 & 0.5402 & -2.5952 & -2.4542 & -1.0366 & 1.3733 & -3.2743 & 0.6175 \\
\hline $\mathrm{H}$ & 7.1284 & 2.2632 & -1.0295 & -3.5311 & -5.2288 & -1.8832 & 1.6136 & -6.2256 & 1.3087 \\
\hline $\mathrm{H}$ & 6.8633 & -0.0997 & -1.6366 & -1.4228 & -6.1060 & -1.1201 & -0.6376 & -6.4658 & 0.4478 \\
\hline C & 3.4873 & 1.9325 & -3.6018 & -3.2466 & -4.5415 & 2.5721 & 1.7071 & -5.3142 & -3.0879 \\
\hline $\mathrm{O}$ & 5.5948 & 3.7389 & 0.4557 & -4.3848 & -2.7397 & -1.9813 & 3.0374 & -3.9534 & 1.5753 \\
\hline $\mathrm{O}$ & 7.0187 & 1.1256 & 0.7005 & -2.0433 & -4.2311 & -2.9230 & 0.3884 & -4.9422 & 2.3860 \\
\hline $\mathrm{O}$ & 5.1239 & -0.3474 & -0.5397 & -0.5863 & -4.2707 & -0.6652 & -0.9010 & -4.4525 & 0.0541 \\
\hline $\mathrm{H}$ & 2.6920 & 2.6735 & -3.4554 & -4.3388 & -4.4404 & 2.6611 & 2.8016 & -5.3934 & -3.1628 \\
\hline $\mathrm{O}$ & 2.9957 & 0.8165 & -4.3368 & -2.6070 & -3.8227 & 3.6149 & 1.2051 & -4.4917 & -4.1278 \\
\hline
\end{tabular}




\begin{tabular}{|c|c|c|c|c|c|c|c|c|c|}
\hline $\mathrm{H}$ & 4.2807 & 2.3807 & -4.2111 & -2.9918 & -5.5954 & 2.7173 & 1.2856 & -6.3113 & -3.2451 \\
\hline $\mathrm{H}$ & 4.7838 & 4.2276 & 0.7029 & -5.2608 & -2.8859 & -1.5687 & 3.8642 & -4.2967 & 1.1790 \\
\hline $\mathrm{C}$ & 7.9164 & 2.0057 & 1.3800 & -2.8372 & -4.2811 & -4.1090 & 1.1060 & -5.2613 & 3.5796 \\
\hline $\mathrm{C}$ & 1.7652 & 0.3248 & -3.8118 & -3.1909 & -2.5448 & 3.8540 & 1.9723 & -3.3013 & -4.2940 \\
\hline $\mathrm{O}$ & 4.9971 & -4.0071 & -0.6148 & 2.8627 & -5.4609 & -0.2021 & -4.3498 & -4.1623 & -1.1720 \\
\hline $\mathrm{C}$ & 4.7649 & -2.6742 & -1.1054 & 1.5335 & -5.0660 & 0.2028 & -2.9155 & -4.0513 & -1.2473 \\
\hline $\mathrm{C}$ & 5.5168 & -1.6800 & -0.2196 & 0.6896 & -4.7953 & -1.0465 & -2.3163 & -4.6659 & 0.0187 \\
\hline $\mathrm{C}$ & 5.1590 & -1.8470 & 1.2608 & 1.3473 & -3.7303 & -1.9353 & -2.8823 & -4.0094 & 1.2841 \\
\hline $\mathrm{C}$ & 5.1754 & -3.3086 & 1.7010 & 2.8049 & -4.1026 & -2.2181 & -4.4084 & -4.0152 & 1.2572 \\
\hline $\mathrm{C}$ & 4.4730 & -4.2138 & 0.6775 & 3.5505 & -4.4633 & -0.9189 & -4.9087 & -3.4544 & -0.0856 \\
\hline $\mathrm{H}$ & 3.6963 & -2.4389 & -1.0478 & 1.5836 & -4.1359 & 0.7766 & -2.6312 & -2.9948 & -1.2871 \\
\hline $\mathrm{H}$ & 6.6005 & -1.8073 & -0.3419 & 0.5675 & -5.7177 & -1.6300 & -2.5435 & -5.7400 & 0.0416 \\
\hline $\mathrm{H}$ & 4.1303 & -1.4747 & 1.3807 & 1.3386 & -2.7873 & -1.3763 & -2.5641 & -2.9588 & 1.2880 \\
\hline $\mathrm{H}$ & 6.2186 & -3.6431 & 1.7785 & 2.8230 & -4.9847 & -2.8735 & -4.7711 & -5.0484 & 1.3547 \\
\hline $\mathrm{H}$ & 4.6638 & -5.2635 & 0.9195 & 4.5348 & -4.8754 & -1.1574 & -5.9922 & -3.5860 & -0.1628 \\
\hline $\mathrm{C}$ & 5.2206 & -2.6392 & -2.5562 & 0.9966 & -6.1730 & 1.1121 & -2.4772 & -4.7474 & -2.5272 \\
\hline $\mathrm{O}$ & 6.0614 & -1.1172 & 2.0763 & 0.6599 & -3.5953 & -3.1681 & -2.4205 & -4.6944 & 2.4342 \\
\hline $\mathrm{O}$ & 4.5072 & -3.4905 & 2.9453 & 3.5354 & -3.0350 & -2.8242 & -4.9791 & -3.1998 & 2.2732 \\
\hline $\mathrm{O}$ & 3.0920 & -3.9382 & 0.7182 & 3.6782 & -3.2976 & -0.1400 & -4.5615 & -2.0931 & -0.1429 \\
\hline $\mathrm{H}$ & 5.2959 & -1.5916 & -2.8798 & -0.0731 & -6.3325 & 0.9308 & -1.4010 & -4.9576 & -2.4692 \\
\hline $\mathrm{O}$ & 4.3490 & -3.3586 & -3.4199 & 1.2115 & -5.8724 & 2.4892 & -2.7819 & -3.9961 & -3.6961 \\
\hline $\mathrm{H}$ & 6.2064 & -3.1112 & -2.6291 & 1.5335 & -7.1007 & 0.8888 & -3.0156 & -5.6979 & -2.6112 \\
\hline $\mathrm{H}$ & 6.2156 & -0.2482 & 1.6490 & -0.3004 & -3.5796 & -2.9786 & -1.4417 & -4.6851 & 2.4108 \\
\hline $\mathrm{C}$ & 5.3274 & -3.3210 & 4.1041 & 3.3811 & -2.9094 & -4.2430 & -5.0918 & -3.8043 & 3.5630 \\
\hline $\mathrm{C}$ & 3.1800 & -2.6142 & -3.7426 & 0.1383 & -5.0931 & 3.0132 & -1.8830 & -2.9071 & -3.8934 \\
\hline $\mathrm{O}$ & 0.4124 & -6.2029 & -0.3767 & 5.8587 & -1.6298 & 2.2478 & -5.8737 & 0.7438 & -2.0422 \\
\hline $\mathrm{C}$ & 1.3734 & -5.1412 & -0.5389 & 4.6856 & -2.3051 & 1.7582 & -5.0274 & -0.3924 & -1.7811 \\
\hline $\mathrm{C}$ & 2.1741 & -5.0292 & 0.7617 & 4.9567 & -2.9646 & 0.3974 & -5.5488 & -1.1480 & -0.5568 \\
\hline $\mathrm{C}$ & 1.2527 & -4.7528 & 1.9553 & 5.6663 & -2.0437 & -0.6005 & -5.8043 & -0.2476 & 0.6575 \\
\hline $\mathrm{C}$ & 0.1600 & -5.8150 & 2.0123 & 6.8514 & -1.3493 & 0.0599 & -6.5798 & 1.0059 & 0.2658 \\
\hline $\mathrm{C}$ & -0.5294 & -5.9640 & 0.6459 & 6.3562 & -0.6625 & 1.3455 & -5.9314 & 1.6548 & -0.9692 \\
\hline $\mathrm{H}$ & 0.8518 & -4.1940 & -0.7253 & 3.8836 & -1.5719 & 1.6468 & -4.0069 & -0.0528 & -1.5851 \\
\hline $\mathrm{H}$ & 2.7093 & -5.9718 & 0.9379 & 5.5649 & -3.8692 & 0.5376 & -6.4872 & -1.6559 & -0.8168 \\
\hline $\mathrm{H}$ & 0.7727 & -3.7763 & 1.7891 & 4.9578 & -1.2611 & -0.8880 & -4.8312 & 0.0722 & 1.0452 \\
\hline $\mathrm{H}$ & 0.6221 & -6.7815 & 2.2561 & 7.6254 & -2.0790 & 0.3335 & -7.6147 & 0.7345 & 0.0132 \\
\hline $\mathrm{H}$ & -1.1812 & -6.8420 & 0.6655 & 7.1806 & -0.1550 & 1.8552 & -6.5334 & 2.5011 & -1.3132 \\
\hline $\mathrm{C}$ & 2.2262 & -5.4941 & -1.7384 & 4.2623 & -3.3458 & 2.7745 & -5.0361 & -1.2602 & -3.0205 \\
\hline $\mathrm{O}$ & 1.9852 & -4.7701 & 3.1659 & 6.0732 & -2.7836 & -1.7415 & -6.5181 & -0.9715 & 1.6441 \\
\hline $\mathrm{O}$ & -0.8493 & -5.5011 & 2.9688 & 7.4087 & -0.3366 & -0.7677 & -6.5764 & 2.0057 & 1.2779 \\
\hline $\mathrm{H}$ & 3.1157 & -4.8557 & -1.7802 & 3.6222 & -4.0912 & 2.2790 & -4.6051 & -2.2450 & -2.7928 \\
\hline $\mathrm{O}$ & 1.4414 & -5.3271 & -2.9119 & 3.5473 & -2.7142 & 3.8247 & -4.2856 & -0.6128 & -4.0378 \\
\hline $\mathrm{H}$ & 2.5551 & -6.5420 & -1.6401 & 5.1562 & -3.8605 & 3.1626 & -6.0806 & -1.4036 & -3.3421 \\
\hline $\mathrm{H}$ & 2.7779 & -4.2087 & 3.0465 & 5.2575 & -2.9049 & -2.2706 & -5.9406 & -1.7149 & 1.9175 \\
\hline $\mathrm{C}$ & -0.5998 & -6.0027 & 4.2865 & 8.4116 & -0.7799 & -1.6849 & -7.4500 & 1.7757 & 2.3857 \\
\hline $\mathrm{C}$ & 2.1035 & -5.8410 & -4.0589 & 3.1202 & -3.6603 & 4.7958 & -4.4671 & -1.2430 & -5.2972 \\
\hline $\mathrm{H}$ & -0.7351 & -2.0966 & -4.2551 & 1.0267 & 0.5128 & 4.3630 & -1.1904 & 1.8030 & -5.1103 \\
\hline $\mathrm{H}$ & -0.9600 & -2.1369 & -2.4906 & 1.6263 & 0.6676 & 2.6966 & -1.1570 & 1.8910 & -3.3358 \\
\hline $\mathrm{H}$ & 0.1531 & -3.3033 & -3.2656 & 2.2983 & -0.5959 & 3.7549 & -2.4122 & 0.9087 & -4.1490 \\
\hline $\mathrm{H}$ & -2.8793 & 0.2858 & -4.6001 & -1.0018 & 3.1790 & 4.4778 & 0.8299 & 3.4855 & -4.3385 \\
\hline $\mathrm{H}$ & -2.8538 & 0.0902 & -2.8346 & -0.5303 & 2.7165 & 2.8289 & 0.5061 & 3.2525 & -2.5996 \\
\hline $\mathrm{H}$ & -2.9750 & -1.3569 & -3.8755 & 0.7226 & 2.8467 & 4.0941 & -0.4524 & 4.4310 & -3.5114 \\
\hline $\mathrm{H}$ & -3.2841 & 2.7152 & -5.9254 & -3.4383 & 4.3678 & 5.3789 & 5.0694 & 4.1326 & -4.8908 \\
\hline $\mathrm{H}$ & -4.6468 & 1.8598 & -5.1380 & -2.1576 & 5.2625 & 4.5013 & 3.9169 & 4.9760 & -3.8086 \\
\hline $\mathrm{H}$ & -4.7275 & 3.6302 & -5.3995 & -3.8493 & 5.8461 & 4.4587 & 5.6614 & 5.3967 & -3.7743 \\
\hline $\mathrm{H}$ & 0.1206 & 2.3239 & -5.9830 & -2.5464 & 0.6880 & 5.1331 & 1.7334 & 1.3613 & -3.6666 \\
\hline $\mathrm{H}$ & -1.3024 & 2.2859 & -4.8905 & -2.5168 & 0.2741 & 3.4073 & 2.4283 & 1.0497 & -2.0681 \\
\hline
\end{tabular}




\begin{tabular}{|c|c|c|c|c|c|c|c|c|c|}
\hline $\mathrm{H}$ & -0.9056 & 3.7769 & -5.7827 & -2.8728 & 1.9523 & 3.9030 & 3.2069 & 2.2449 & -3.1470 \\
\hline $\mathrm{H}$ & 1.3877 & -0.4193 & -4.5203 & -2.6653 & -2.1086 & 4.7075 & 1.4689 & -2.6993 & -5.0553 \\
\hline $\mathrm{H}$ & 1.9071 & -0.1609 & -2.8404 & -3.0951 & -1.8864 & 2.9862 & 2.0501 & -2.7350 & -3.3625 \\
\hline $\mathrm{H}$ & 1.0383 & 1.1379 & -3.7002 & -4.2611 & -2.6328 & 4.0814 & 2.9942 & -3.5395 & -4.6246 \\
\hline $\mathrm{H}$ & 2.6236 & -3.1886 & -4.4855 & 0.4138 & -4.7900 & 4.0261 & -2.0642 & -2.5197 & -4.8983 \\
\hline $\mathrm{H}$ & 2.5345 & -2.4687 & -2.8658 & -0.0478 & -4.1975 & 2.4099 & -2.0592 & -2.0963 & -3.1755 \\
\hline $\mathrm{H}$ & 3.4469 & -1.6294 & -4.1473 & -0.7882 & -5.6796 & 3.0450 & -0.8427 & -3.2483 & -3.8149 \\
\hline $\mathrm{H}$ & 1.4724 & -5.6178 & -4.9236 & 2.4852 & -3.1303 & 5.5105 & -3.7949 & -0.7521 & -6.0062 \\
\hline $\mathrm{H}$ & 3.0882 & -5.3722 & -4.1904 & 2.5498 & -4.4729 & 4.3238 & -4.2242 & -2.3135 & -5.2435 \\
\hline $\mathrm{H}$ & 2.2383 & -6.9311 & -3.9789 & 3.9802 & -4.0985 & 5.3258 & -5.5066 & -1.1356 & -5.6442 \\
\hline $\mathrm{H}$ & -7.1950 & -4.6322 & 2.9651 & 5.6993 & 5.7444 & -2.3368 & -2.5485 & 6.9419 & 3.0775 \\
\hline $\mathrm{H}$ & -6.8823 & -5.9676 & 1.8166 & 6.7344 & 5.6927 & -0.8785 & -3.6853 & 7.4002 & 1.7738 \\
\hline $\mathrm{H}$ & -5.6315 & -5.5154 & 3.0119 & 6.6285 & 4.2591 & -1.9412 & -3.9789 & 5.9137 & 2.7275 \\
\hline $\mathrm{H}$ & -7.0266 & 1.9972 & 3.9368 & -0.6204 & 4.6307 & -4.8168 & 1.9241 & 3.2299 & 5.1204 \\
\hline $\mathrm{H}$ & -8.2659 & 1.2143 & 2.9097 & 0.3101 & 6.0170 & -4.1701 & 1.5520 & 4.9566 & 4.8208 \\
\hline $\mathrm{H}$ & -6.9488 & 0.2334 & 3.6185 & 1.0386 & 4.3839 & -4.1836 & 0.3772 & 3.6933 & 4.3399 \\
\hline $\mathrm{H}$ & -2.0439 & 6.2527 & 3.6364 & -6.2525 & 1.9526 & -4.1949 & 6.8944 & -0.4765 & 4.1886 \\
\hline $\mathrm{H}$ & -3.6794 & 6.5191 & 2.9545 & -6.1811 & 3.7045 & -3.8286 & 7.8528 & 0.9464 & 3.6803 \\
\hline $\mathrm{H}$ & -3.1826 & 4.8747 & 3.4646 & -4.6705 & 2.7962 & -4.1176 & 6.1905 & 1.1687 & 4.3067 \\
\hline $\mathrm{H}$ & 4.3276 & 6.3906 & 2.3561 & -7.9204 & -3.7257 & -2.0102 & 6.1260 & -6.0630 & 1.2748 \\
\hline $\mathrm{H}$ & 3.5062 & 7.4693 & 1.1911 & -8.8027 & -2.7922 & -0.7638 & 7.1844 & -5.4373 & -0.0253 \\
\hline $\mathrm{H}$ & 2.5432 & 6.5605 & 2.3933 & -7.9555 & -1.9305 & -2.0841 & 6.8936 & -4.4470 & 1.4378 \\
\hline $\mathrm{H}$ & 8.3834 & 1.4165 & 2.1734 & -2.1428 & -4.1751 & -4.9465 & 0.4406 & -5.0197 & 4.4125 \\
\hline $\mathrm{H}$ & 8.6905 & 2.3675 & 0.6892 & -3.3520 & -5.2501 & -4.1805 & 1.3501 & -6.3326 & 3.6070 \\
\hline $\mathrm{H}$ & 7.3813 & 2.8602 & 1.8024 & -3.5694 & -3.4715 & -4.1227 & 2.0243 & -4.6733 & 3.6464 \\
\hline $\mathrm{H}$ & 4.7011 & -3.5688 & 4.9645 & 4.0818 & -2.1319 & -4.5557 & -5.6327 & -3.0896 & 4.1875 \\
\hline $\mathrm{H}$ & 6.1875 & -4.0041 & 4.0691 & 3.6289 & -3.8567 & -4.7408 & -5.6575 & -4.7446 & 3.4981 \\
\hline $\mathrm{H}$ & 5.6861 & -2.2905 & 4.1802 & 2.3588 & -2.6194 & -4.4944 & -4.1040 & -3.9974 & 3.9867 \\
\hline $\mathrm{H}$ & -1.4850 & -5.7623 & 4.8803 & 8.7908 & 0.1150 & -2.1843 & -7.4548 & 2.7005 & 2.9683 \\
\hline $\mathrm{H}$ & -0.4585 & -7.0922 & 4.2645 & 9.2320 & -1.2717 & -1.1440 & -8.4672 & 1.5607 & 2.0308 \\
\hline $\mathrm{H}$ & 0.2871 & -5.5283 & 4.7147 & 7.9880 & -1.4745 & -2.4152 & -7.0977 & 0.9397 & 2.9952 \\
\hline $\mathrm{C}$ & 2.5594 & 3.4813 & 3.2233 & 1.4746 & -1.2324 & 0.5514 & -3.2090 & 0.8057 & 2.7822 \\
\hline $\mathrm{C}$ & -0.4367 & 0.9309 & -0.1300 & -1.1347 & -0.8512 & -4.1097 & 2.0179 & 0.5260 & 2.8832 \\
\hline $\mathrm{C}$ & 3.8215 & 0.9992 & 3.0620 & -1.1432 & -0.6785 & 1.2376 & -2.7446 & -1.3472 & 4.4802 \\
\hline C & 3.9543 & 3.4027 & 3.2321 & 0.9297 & -1.8027 & 1.7009 & -4.0662 & 0.5697 & 3.8593 \\
\hline $\mathrm{C}$ & 1.2483 & -1.1466 & 0.6131 & -3.0792 & 0.2173 & -2.4671 & 1.4240 & -1.7201 & 4.3953 \\
\hline C & -0.1138 & -0.0578 & -1.0591 & -2.4717 & -0.9194 & -4.4985 & 2.8766 & 0.1515 & 3.9190 \\
\hline $\mathrm{C}$ & 4.5906 & 2.1646 & 3.1567 & -0.3911 & -1.5332 & 2.0503 & -3.8374 & -0.5059 & 4.7184 \\
\hline $\mathrm{C}$ & 0.7260 & -1.1085 & -0.6807 & -3.4522 & -0.3689 & -3.6740 & 2.5830 & -0.9883 & 4.6703 \\
\hline $\mathrm{C}$ & -3.6988 & 1.9386 & 4.1217 & 3.1899 & 2.0558 & -4.7011 & 0.4438 & -0.9059 & -2.2678 \\
\hline $\mathrm{C}$ & -0.2454 & 3.4257 & 3.9990 & 2.7614 & 0.2234 & -1.4566 & -1.9047 & 0.7019 & 0.1750 \\
\hline $\mathrm{C}$ & -1.8067 & 1.3992 & 2.7465 & 1.2664 & 1.0105 & -3.7111 & 0.6044 & -0.6192 & 0.1028 \\
\hline $\mathrm{C}$ & -1.5569 & 2.9243 & 4.6002 & 3.2840 & 1.2513 & -2.4475 & -1.4344 & -0.0712 & -1.0516 \\
\hline $\mathrm{C}$ & 1.5736 & -0.1763 & 2.9203 & -1.3977 & 0.8994 & -0.7244 & -0.7358 & -2.0792 & 3.1179 \\
\hline $\mathrm{C}$ & 1.7852 & 2.3152 & 3.1468 & 0.7285 & -0.3677 & -0.2598 & -2.1293 & -0.0573 & 2.5205 \\
\hline $\mathrm{C}$ & 0.0542 & 0.8819 & 1.1817 & -0.7427 & -0.2155 & -2.9211 & 0.8838 & -0.2296 & 2.5705 \\
\hline $\mathrm{C}$ & 2.4298 & 1.0599 & 3.0581 & -0.5990 & -0.0674 & 0.1084 & -1.8970 & -1.1507 & 3.3909 \\
\hline $\mathrm{C}$ & 0.9165 & -0.1660 & 1.5549 & -1.7368 & 0.3065 & -2.0764 & 0.5680 & -1.3542 & 3.3513 \\
\hline $\mathrm{C}$ & -0.4894 & 1.9206 & 2.1418 & 0.7603 & -0.0727 & -2.7365 & 0.1136 & 0.1710 & 1.3319 \\
\hline $\mathrm{N}$ & -2.4494 & 2.4273 & 3.5578 & 2.7263 & 1.0231 & -3.7790 & 0.0266 & -0.0874 & -1.1362 \\
\hline $\mathrm{N}$ & 0.3794 & 2.3307 & 3.2604 & 1.2926 & 0.2672 & -1.3916 & -1.3533 & 0.0639 & 1.3607 \\
\hline $\mathrm{H}$ & 2.0636 & 4.4453 & 3.2369 & 2.4713 & -1.5052 & 0.2511 & -3.3930 & 1.6553 & 2.1344 \\
\hline $\mathrm{H}$ & -1.0741 & 1.7630 & -0.4162 & -0.3659 & -1.2890 & -4.7407 & 2.2130 & 1.4310 & 2.3189 \\
\hline $\mathrm{H}$ & 4.3153 & 0.0354 & 3.0050 & -2.1775 & -0.4734 & 1.4805 & -2.5605 & -2.1973 & 5.1321 \\
\hline $\mathrm{H}$ & 4.5408 & 4.3148 & 3.2731 & 1.5483 & -2.4435 & 2.3212 & -4.9066 & 1.2379 & 4.0233 \\
\hline $\mathrm{H}$ & 1.9225 & -1.9476 & 0.8977 & -3.8402 & 0.6218 & -1.8123 & 1.1724 & -2.5900 & 4.9956 \\
\hline
\end{tabular}




\begin{tabular}{rrrrrrrrrr}
$\mathrm{H}$ & -0.5055 & 0.0026 & -2.0691 & -2.7412 & -1.4133 & -5.4269 & 3.7553 & 0.7548 & 4.1222 \\
$\mathrm{H}$ & 5.6734 & 2.1012 & 3.1460 & -0.8351 & -1.9961 & 2.9232 & -4.4993 & -0.6939 & 5.5584 \\
$\mathrm{H}$ & 0.9862 & -1.8885 & -1.3889 & -4.5025 & -0.4257 & -3.9408 & 3.2395 & -1.2972 & 5.4790 \\
$\mathrm{H}$ & -4.2117 & 2.7577 & 4.6375 & 4.2823 & 2.0068 & -4.7831 & 0.0279 & -0.4970 & -3.1955 \\
$\mathrm{H}$ & -4.3469 & 1.5741 & 3.3208 & 2.7563 & 1.8865 & -5.6932 & 1.5312 & -0.9143 & -2.3531 \\
$\mathrm{H}$ & -3.5371 & 1.1155 & 4.8450 & 2.9143 & 3.0696 & -4.3585 & 0.0968 & -1.9484 & -2.1687 \\
$\mathrm{H}$ & 0.4262 & 3.7372 & 4.8030 & 3.1698 & 0.4437 & -0.4679 & -2.9899 & 0.6894 & 0.2125 \\
$\mathrm{H}$ & -0.4358 & 4.2924 & 3.3486 & 3.1031 & -0.7799 & -1.7554 & -1.5722 & 1.7498 & 0.0989 \\
$\mathrm{H}$ & -2.4910 & 1.1239 & 1.9352 & 0.8784 & 0.8001 & -4.7125 & 1.6964 & -0.5624 & 0.0345 \\
$\mathrm{H}$ & -1.5810 & 0.4855 & 3.3311 & 0.8545 & 1.9841 & -3.3808 & 0.3141 & -1.6748 & 0.2464 \\
$\mathrm{H}$ & -1.3242 & 2.1331 & 5.3398 & 3.0371 & 2.2605 & -2.0822 & -1.8430 & -1.0962 & -0.9776 \\
$\mathrm{H}$ & -2.0616 & 3.7434 & 5.1254 & 4.3773 & 1.1719 & -2.5028 & -1.8333 & 0.3814 & -1.9637 \\
$\mathrm{H}$ & 2.1800 & -1.0808 & 3.0349 & -2.3248 & 1.1523 & -0.2019 & -0.8027 & -2.9613 & 3.7563 \\
$\mathrm{H}$ & 0.8031 & -0.1646 & 3.7027 & -0.8249 & 1.8265 & -0.8536 & -0.7742 & -2.4195 & 2.0778 \\
$\mathrm{H}$ & -0.7438 & 2.8150 & 1.5500 & 1.2064 & -1.0313 & -3.0494 & 0.3577 & 1.2248 & 1.1361 \\
\hline
\end{tabular}

\begin{tabular}{|c|c|c|c|c|c|c|}
\hline & & & \\
\hline atom & $\mathbf{x}$ & $\mathbf{y}$ & $\mathbf{Z}$ & $\mathbf{x}$ & $\mathbf{y}$ & $\mathbf{z}$ \\
\hline $\mathrm{O}$ & -4.9115 & -3.0311 & -1.9585 & 5.1457 & 2.7783 & 1.8700 \\
\hline $\mathrm{C}$ & -3.4979 & -3.2423 & -1.7507 & 4.6738 & 1.4191 & 1.7650 \\
\hline $\mathrm{C}$ & -3.3216 & -4.3352 & -0.6920 & 5.5960 & 0.6419 & 0.8215 \\
\hline $\mathrm{C}$ & -4.0444 & -3.9730 & 0.6097 & 5.7307 & 1.3364 & -0.5390 \\
\hline $\mathrm{C}$ & -5.5111 & -3.6813 & 0.3062 & 6.1270 & 2.7968 & -0.3525 \\
\hline $\mathrm{C}$ & -5.6032 & -2.6195 & -0.8025 & 5.1655 & 3.4663 & 0.6415 \\
\hline $\mathrm{H}$ & -3.0411 & -2.3168 & -1.3823 & 3.6635 & 1.4158 & 1.3411 \\
\hline $\mathrm{H}$ & -3.7339 & -5.2757 & -1.0817 & 6.5894 & 0.5401 & 1.2794 \\
\hline $\mathrm{H}$ & -3.5868 & -3.0532 & 0.9993 & 4.7412 & 1.3170 & -1.0127 \\
\hline $\mathrm{H}$ & -6.0000 & -4.6007 & -0.0447 & 7.1483 & 2.8573 & 0.0482 \\
\hline $\mathrm{H}$ & -6.6457 & -2.4731 & -1.1012 & 5.4985 & 4.4848 & 0.8633 \\
\hline $\mathrm{C}$ & -2.8960 & -3.6280 & -3.0949 & 4.6542 & 0.8318 & 3.1696 \\
\hline $\mathrm{O}$ & -1.9369 & -4.4916 & -0.3690 & 5.0220 & -0.6431 & 0.5719 \\
\hline $\mathrm{O}$ & -3.9553 & -5.0264 & 1.5502 & 6.6905 & 0.6808 & -1.3480 \\
\hline $\mathrm{O}$ & -6.2200 & -3.1570 & 1.4225 & 6.0342 & 3.5513 & -1.5567 \\
\hline $\mathrm{O}$ & -5.0494 & -1.4256 & -0.3000 & 3.8775 & 3.4728 & 0.0628 \\
\hline $\mathrm{H}$ & -1.9431 & -4.1463 & -2.9392 & 4.6308 & -0.2640 & 3.1083 \\
\hline $\mathrm{O}$ & -2.7222 & -2.5123 & -3.9634 & 3.5681 & 1.3132 & 3.9543 \\
\hline $\mathrm{H}$ & -3.5876 & -4.3143 & -3.5965 & 5.5740 & 1.1349 & 3.6826 \\
\hline $\mathrm{H}$ & -3.0041 & -5.2228 & 1.6876 & 6.3966 & -0.2496 & -1.4521 \\
\hline $\mathrm{C}$ & -6.7544 & -4.1275 & 2.3264 & 7.1837 & 3.4975 & -2.4049 \\
\hline $\mathrm{C}$ & -1.5562 & -1.7564 & -3.6471 & 2.3430 & 0.6524 & 3.6488 \\
\hline $\mathrm{O}$ & -6.0690 & 2.0067 & -1.0435 & 1.4564 & 6.1981 & 0.4130 \\
\hline $\mathrm{C}$ & -5.4283 & 0.7428 & -1.2923 & 2.1742 & 5.0193 & 0.8209 \\
\hline $\mathrm{C}$ & -5.8522 & -0.2495 & -0.2103 & 3.2477 & 4.7200 & -0.2246 \\
\hline $\mathrm{C}$ & -5.5892 & 0.2946 & 1.1993 & 2.6327 & 4.5484 & -1.6179 \\
\hline $\mathrm{C}$ & -6.0576 & 1.7385 & 1.3647 & 1.6533 & 5.6728 & -1.9511 \\
\hline $\mathrm{C}$ & -5.6422 & 2.5994 & 0.1630 & 0.7181 & 5.9994 & -0.7734 \\
\hline $\mathrm{H}$ & -4.3417 & 0.8641 & -1.2311 & 1.4886 & 4.1656 & 0.8564 \\
\hline $\mathrm{H}$ & -6.9178 & -0.4941 & -0.3128 & 3.9860 & 5.5320 & -0.2532 \\
\hline $\mathrm{H}$ & -4.4985 & 0.2772 & 1.3347 & 2.0707 & 3.6028 & -1.6064 \\
\hline $\mathrm{H}$ & -7.1543 & 1.7500 & 1.4271 & 2.2297 & 6.5813 & -2.1720 \\
\hline $\mathrm{H}$ & -6.1351 & 3.5747 & 0.2162 & 0.2031 & 6.9447 & -0.9694 \\
\hline $\mathrm{C}$ & -5.8114 & 0.3006 & -2.6953 & 2.7349 & 5.2756 & 2.2113 \\
\hline $\mathrm{O}$ & -6.2389 & -0.5042 & 2.1745 & 3.6436 & 4.5234 & -2.6149 \\
\hline $\mathrm{O}$ & -5.4977 & 2.3606 & 2.5173 & 0.8263 & 5.3363 & -3.0621 \\
\hline $\mathrm{O}$ & -4.2407 & 2.7432 & 0.1938 & -0.2069 & 4.9488 & -0.6276 \\
\hline $\mathrm{H}$ & -5.5382 & -0.7570 & -2.8198 & 3.5048 & 4.5234 & 2.4309 \\
\hline $\mathrm{O}$ & -5.1943 & 1.0952 & -3.7006 & 1.7270 & 5.2519 & 3.2149 \\
\hline
\end{tabular}




\begin{tabular}{|c|c|c|c|c|c|c|}
\hline $\mathrm{H}$ & -6.8941 & 0.4103 & -2.8211 & 3.1873 & 6.2729 & 2.2356 \\
\hline $\mathrm{H}$ & -6.1141 & -1.4448 & 1.9238 & 4.4037 & 4.0154 & -2.2607 \\
\hline $\mathrm{C}$ & -6.1617 & 2.0842 & 3.7528 & 1.3660 & 5.6726 & -4.3427 \\
\hline C & -3.8298 & 0.7439 & -3.9093 & 1.3376 & 3.9261 & 3.5616 \\
\hline $\mathrm{O}$ & -2.4126 & 5.5161 & -1.3814 & -3.4939 & 5.1160 & 1.0103 \\
\hline C & -3.0079 & 4.2036 & -1.3393 & -2.0713 & 4.8882 & 0.9472 \\
\hline $\mathrm{C}$ & -3.6865 & 4.0448 & 0.0249 & -1.5850 & 5.2797 & -0.4510 \\
\hline C & -2.6635 & 4.2022 & 1.1561 & -2.3308 & 4.4941 & -1.5343 \\
\hline C & -1.9357 & 5.5354 & 1.0074 & -3.8392 & 4.6409 & -1.3503 \\
\hline $\mathrm{C}$ & -1.3942 & 5.6996 & -0.4246 & -4.2339 & 4.3298 & 0.1043 \\
\hline $\mathrm{H}$ & -2.2274 & 3.4399 & -1.4425 & -1.8596 & 3.8253 & 1.1152 \\
\hline $\mathrm{H}$ & -4.4649 & 4.8118 & 0.1346 & -1.7479 & 6.3545 & -0.6065 \\
\hline $\mathrm{H}$ & -1.9266 & 3.3925 & 1.0483 & -2.0681 & 3.4396 & -1.3926 \\
\hline $\mathrm{H}$ & -2.6478 & 6.3504 & 1.1961 & -4.1259 & 5.6796 & -1.5658 \\
\hline $\mathrm{H}$ & -1.0320 & 6.7228 & -0.5609 & -5.2841 & 4.5914 & 0.2634 \\
\hline C & -3.9539 & 4.1021 & -2.5158 & -1.4399 & 5.7054 & 2.0530 \\
\hline $\mathrm{O}$ & -3.2946 & 4.1463 & 2.4223 & -1.9625 & 4.9429 & -2.8246 \\
\hline $\mathrm{O}$ & -0.8196 & 5.6491 & 1.8854 & -4.5876 & 3.7509 & -2.1756 \\
\hline $\mathrm{O}$ & -0.3542 & 4.7709 & -0.6153 & -4.0203 & 2.9579 & 0.3348 \\
\hline $\mathrm{H}$ & -4.6005 & 3.2234 & -2.4122 & -0.3537 & 5.7556 & 1.9171 \\
\hline $\mathrm{O}$ & -3.1740 & 4.0041 & -3.7009 & -1.7566 & 5.0932 & 3.2964 \\
\hline $\mathrm{H}$ & -4.5897 & 5.0028 & -2.5444 & -1.8452 & 6.7303 & 2.0127 \\
\hline $\mathrm{H}$ & -3.9354 & 3.4062 & 2.4100 & -0.9840 & 4.9569 & -2.8658 \\
\hline $\mathrm{C}$ & -1.1156 & 6.1259 & 3.2024 & -4.8135 & 4.1938 & -3.5180 \\
\hline C & -3.9877 & 4.0429 & -4.8645 & -1.3495 & 5.8977 & 4.3940 \\
\hline $\mathrm{O}$ & 2.5956 & 4.5913 & -2.7649 & -5.3479 & 0.4810 & 2.6711 \\
\hline C & 1.2725 & 4.2455 & -2.3027 & -4.3788 & 1.4001 & 2.1242 \\
\hline C & 0.8475 & 5.2457 & -1.2264 & -5.0408 & 2.2269 & 1.0197 \\
\hline C & 1.8828 & 5.3897 & -0.1086 & -5.7448 & 1.3618 & -0.0304 \\
\hline C & 3.2808 & 5.6074 & -0.6705 & -6.6503 & 0.3283 & 0.6284 \\
\hline C & 3.5713 & 4.5554 & -1.7528 & -5.8689 & -0.4179 & 1.7231 \\
\hline $\mathrm{H}$ & 1.2885 & 3.2413 & -1.8658 & -3.5464 & 0.8366 & 1.6891 \\
\hline $\mathrm{H}$ & 0.6832 & 6.2266 & -1.6928 & -5.7718 & 2.9129 & 1.4689 \\
\hline $\mathrm{H}$ & 1.8967 & 4.4416 & 0.4446 & -4.9685 & 0.8122 & -0.5712 \\
\hline $\mathrm{H}$ & 3.3532 & 6.6070 & -1.1208 & -7.5152 & 0.8253 & 1.0892 \\
\hline $\mathrm{H}$ & 4.5317 & 4.7739 & -2.2292 & -6.5416 & -1.0921 & 2.2618 \\
\hline C & 0.3322 & 4.2813 & -3.5023 & -3.8790 & 2.2856 & 3.2609 \\
\hline $\mathrm{O}$ & 1.5351 & 6.4688 & 0.7453 & -6.4965 & 2.1794 & -0.9131 \\
\hline $\mathrm{O}$ & 4.2937 & 5.4406 & 0.3164 & -7.0976 & -0.6743 & -0.2804 \\
\hline $\mathrm{O}$ & 3.5807 & 3.2721 & -1.1598 & -4.8094 & -1.1355 & 1.1226 \\
\hline $\mathrm{H}$ & -0.6989 & 4.4249 & -3.1528 & -3.4761 & 3.2192 & 2.8465 \\
\hline $\mathrm{O}$ & 0.4232 & 3.1212 & -4.3205 & -2.9109 & 1.6488 & 4.0856 \\
\hline $\mathrm{H}$ & 0.6108 & 5.1356 & -4.1296 & -4.7325 & 2.5293 & 3.9035 \\
\hline $\mathrm{H}$ & 0.6903 & 6.2224 & 1.1816 & -5.8538 & 2.7405 & -1.3958 \\
\hline C & 4.5587 & 6.5875 & 1.1283 & -8.2219 & -0.3147 & -1.0893 \\
\hline C & -0.3091 & 2.0226 & -3.7868 & -1.6113 & 1.6567 & 3.5037 \\
\hline $\mathrm{O}$ & 6.1494 & 0.7230 & -1.6916 & -3.8452 & -4.5866 & 1.8525 \\
\hline $\mathrm{C}$ & 5.0108 & 1.5404 & -2.0230 & -3.9771 & -3.1738 & 2.0964 \\
\hline C & 4.8252 & 2.6024 & -0.9406 & -4.8747 & -2.5614 & 1.0241 \\
\hline C & 4.7003 & 1.9748 & 0.4570 & -4.3871 & -2.8827 & -0.3962 \\
\hline C & 5.7547 & 0.8917 & 0.7064 & -3.9363 & -4.3360 & -0.5700 \\
\hline C & 5.9414 & -0.0266 & -0.5140 & -3.1623 & -4.8654 & 0.6491 \\
\hline $\mathrm{H}$ & 4.1072 & 0.9211 & -2.0266 & -2.9948 & -2.6949 & 2.0165 \\
\hline $\mathrm{H}$ & 5.6576 & 3.3162 & -0.9421 & -5.9077 & -2.9130 & 1.1374 \\
\hline $\mathrm{H}$ & 3.7151 & 1.4835 & 0.4957 & -3.5058 & -2.2478 & -0.5779 \\
\hline $\mathrm{H}$ & 6.7162 & 1.3867 & 0.9047 & -4.8320 & -4.9614 & -0.6886 \\
\hline
\end{tabular}




\begin{tabular}{|c|c|c|c|c|c|c|}
\hline $\mathrm{H}$ & 6.8393 & -0.6353 & -0.3754 & -3.0987 & -5.9553 & 0.5842 \\
\hline $\mathrm{C}$ & 5.2251 & 2.0833 & -3.4396 & -4.4950 & -2.9976 & 3.5269 \\
\hline $\mathrm{O}$ & 4.8266 & 2.9620 & 1.4648 & -5.4127 & -2.6182 & -1.3410 \\
\hline $\mathrm{O}$ & 5.4083 & 0.0380 & 1.7947 & -3.0720 & -4.4928 & -1.6950 \\
\hline $\mathrm{O}$ & 4.8026 & -0.8414 & -0.6437 & -1.8778 & -4.2935 & 0.6620 \\
\hline $\mathrm{H}$ & 4.9429 & 3.1422 & -3.4990 & -5.2459 & -2.1978 & 3.5688 \\
\hline $\mathrm{O}$ & 4.4986 & 1.3426 & -4.4115 & -3.4511 & -2.7175 & 4.4488 \\
\hline $\mathrm{H}$ & 6.2866 & 1.9958 & -3.6906 & -4.9691 & -3.9320 & 3.8430 \\
\hline $\mathrm{H}$ & 4.3950 & 3.7799 & 1.1476 & -5.9264 & -1.8480 & -1.0187 \\
\hline $\mathrm{C}$ & 5.5944 & 0.5847 & 3.1051 & -3.7208 & -4.5657 & -2.9689 \\
\hline C & 3.1264 & 1.7283 & -4.4399 & -3.0734 & -1.3439 & 4.3992 \\
\hline $\mathrm{O}$ & 4.1212 & -4.0737 & -2.1961 & 1.2268 & -5.4034 & 2.2406 \\
\hline C & 4.0171 & -2.6482 & -2.0180 & 0.0981 & -4.5310 & 2.0377 \\
\hline C & 5.0273 & -2.2152 & -0.9573 & -0.7972 & -5.1698 & 0.9802 \\
\hline C & 4.8349 & -2.9928 & 0.3511 & -0.0338 & -5.4116 & -0.3274 \\
\hline C & 4.8110 & -4.4972 & 0.0910 & 1.2646 & -6.1682 & -0.0656 \\
\hline C & 3.8025 & -4.7969 & -1.0293 & 2.0391 & -5.5202 & 1.0950 \\
\hline $\mathrm{H}$ & 3.0158 & -2.3991 & -1.6520 & 0.4459 & -3.5636 & 1.6603 \\
\hline $\mathrm{H}$ & 6.0466 & -2.3685 & -1.3363 & -1.1726 & -6.1274 & 1.3646 \\
\hline $\mathrm{H}$ & 3.8530 & -2.7040 & 0.7415 & 0.2274 & -4.4286 & -0.7366 \\
\hline $\mathrm{H}$ & 5.8062 & -4.8347 & -0.2304 & 1.0297 & -7.2028 & 0.2191 \\
\hline $\mathrm{H}$ & 3.8343 & -5.8564 & -1.3001 & 2.8860 & -6.1516 & 1.3799 \\
\hline C & 4.2460 & -2.0032 & -3.3753 & -0.5795 & -4.3457 & 3.3852 \\
\hline $\mathrm{O}$ & 5.8573 & -2.6659 & 1.2752 & -0.8385 & -6.1405 & -1.2374 \\
\hline $\mathrm{O}$ & 4.3812 & -5.2641 & 1.2127 & 2.1370 & -6.1600 & -1.1927 \\
\hline $\mathrm{O}$ & 2.5264 & -4.4361 & -0.5482 & 2.4790 & -4.2524 & 0.6661 \\
\hline $\mathrm{H}$ & 4.3530 & -0.9179 & -3.2494 & -1.5435 & -3.8418 & 3.2442 \\
\hline $\mathrm{O}$ & 3.2137 & -2.2941 & -4.3121 & 0.2233 & -3.6261 & 4.3155 \\
\hline $\mathrm{H}$ & 5.1718 & -2.3994 & -3.8063 & -0.7603 & -5.3293 & 3.8318 \\
\hline $\mathrm{H}$ & 5.6947 & -1.7392 & 1.5524 & -1.5832 & -5.5534 & -1.4847 \\
\hline C & 5.3786 & -5.5115 & 2.2082 & 1.9251 & -7.2253 & -2.1241 \\
\hline C & 1.9606 & -1.7172 & -3.9669 & 0.4238 & -2.2658 & 3.9522 \\
\hline $\mathrm{O}$ & -0.6389 & -5.9138 & -1.6780 & 5.4069 & -2.4518 & 1.9866 \\
\hline C & 0.4798 & -5.0100 & -1.7490 & 4.0332 & -2.8874 & 1.9753 \\
\hline C & 1.4529 & -5.3686 & -0.6235 & 3.8493 & -3.8981 & 0.8358 \\
\hline C & 0.7692 & -5.2874 & 0.7433 & 4.2469 & -3.2580 & -0.4911 \\
\hline C & -0.5296 & -6.0864 & 0.7497 & 5.6687 & -2.7196 & -0.4121 \\
\hline C & -1.3919 & -5.7843 & -0.4900 & 5.8060 & -1.7871 & 0.8037 \\
\hline $\mathrm{H}$ & 0.1321 & -3.9790 & -1.6077 & 3.3768 & -2.0279 & 1.7896 \\
\hline $\mathrm{H}$ & 1.8345 & -6.3869 & -0.7768 & 4.4594 & -4.7916 & 1.0239 \\
\hline $\mathrm{H}$ & 0.5114 & -4.2313 & 0.9172 & 3.5734 & -2.4033 & -0.6369 \\
\hline $\mathrm{H}$ & -0.2811 & -7.1560 & 0.7217 & 6.3692 & -3.5547 & -0.2786 \\
\hline $\mathrm{H}$ & -2.1865 & -6.5329 & -0.5613 & 6.8562 & -1.5183 & 0.9515 \\
\hline C & 1.1006 & -5.1404 & -3.1237 & 3.7194 & -3.4641 & 3.3386 \\
\hline $\mathrm{O}$ & 1.6216 & -5.7812 & 1.7615 & 4.1446 & -4.1674 & -1.5716 \\
\hline $\mathrm{O}$ & -1.3180 & -5.7966 & 1.9009 & 6.0118 & -1.9775 & -1.5794 \\
\hline $\mathrm{H}$ & 2.0683 & -4.6225 & -3.1369 & 2.7467 & -3.9717 & 3.3052 \\
\hline $\mathrm{O}$ & 0.2227 & -4.5619 & -4.0764 & 3.6893 & -2.4029 & 4.2812 \\
\hline $\mathrm{H}$ & 1.2714 & -6.2064 & -3.3483 & 4.4913 & -4.2032 & 3.6104 \\
\hline $\mathrm{H}$ & 2.5327 & -5.4838 & 1.5676 & 3.3534 & -4.7263 & -1.4336 \\
\hline C & -1.0458 & -6.6156 & 3.0426 & 6.6766 & -2.7317 & -2.5975 \\
\hline C & 0.7793 & -4.5874 & -5.3833 & 3.3581 & -2.8703 & 5.5816 \\
\hline $\mathrm{H}$ & -1.4689 & -0.9711 & -4.4036 & 1.5918 & 1.0324 & 4.3474 \\
\hline $\mathrm{H}$ & -1.6311 & -1.2778 & -2.6591 & 2.0033 & 0.8677 & 2.6246 \\
\hline $\mathrm{H}$ & -0.6695 & -2.3991 & -3.6712 & 2.4538 & -0.4312 & 3.7710 \\
\hline $\mathrm{H}$ & -3.4722 & 1.3346 & -4.7548 & 0.6380 & 4.0053 & 4.3956 \\
\hline
\end{tabular}




\begin{tabular}{|c|c|c|c|c|c|c|}
\hline $\mathrm{H}$ & -3.2062 & 0.9831 & -3.0365 & 0.8248 & 3.4192 & 2.7322 \\
\hline $\mathrm{H}$ & -3.7351 & -0.3279 & -4.1260 & 2.2113 & 3.3274 & 3.8517 \\
\hline $\mathrm{H}$ & -3.3264 & 3.9127 & -5.7257 & -1.5840 & 5.3419 & 5.3063 \\
\hline $\mathrm{H}$ & -4.7364 & 3.2392 & -4.8494 & -0.2715 & 6.1038 & 4.3546 \\
\hline $\mathrm{H}$ & -4.5073 & 5.0105 & -4.9495 & -1.8941 & 6.8552 & 4.4030 \\
\hline $\mathrm{H}$ & -0.2063 & 1.1953 & -4.4953 & -0.9374 & 1.1770 & 4.2198 \\
\hline $\mathrm{H}$ & 0.0893 & 1.6971 & -2.8137 & -1.5789 & 1.0870 & 2.5624 \\
\hline $\mathrm{H}$ & -1.3689 & 2.2860 & -3.6688 & -1.2814 & 2.6863 & 3.3122 \\
\hline $\mathrm{H}$ & 2.6390 & 1.1357 & -5.2178 & -2.2909 & -1.1946 & 5.1469 \\
\hline $\mathrm{H}$ & 2.6264 & 1.5386 & -3.4796 & -2.6820 & -1.0613 & 3.4116 \\
\hline $\mathrm{H}$ & 3.0152 & 2.7975 & -4.6521 & -3.9253 & -0.6869 & 4.6064 \\
\hline $\mathrm{H}$ & 1.3150 & -1.8058 & -4.8433 & 0.8333 & -1.7575 & 4.8276 \\
\hline $\mathrm{H}$ & 1.4818 & -2.2483 & -3.1342 & 1.1438 & -2.1639 & 3.1301 \\
\hline $\mathrm{H}$ & 2.0686 & -0.6573 & -3.6989 & -0.5209 & -1.7876 & 3.6600 \\
\hline $\mathrm{H}$ & 0.0726 & -4.0775 & -6.0436 & 3.3056 & -1.9951 & 6.2350 \\
\hline $\mathrm{H}$ & 1.7475 & -4.0674 & -5.4073 & 2.3878 & -3.3866 & 5.5779 \\
\hline $\mathrm{H}$ & 0.9218 & -5.6219 & -5.7325 & 4.1275 & -3.5609 & 5.9609 \\
\hline $\mathrm{H}$ & -7.3503 & -3.5725 & 3.0552 & 6.9944 & 4.1964 & -3.2235 \\
\hline $\mathrm{H}$ & -7.4010 & -4.8331 & 1.7871 & 8.0787 & 3.8161 & -1.8526 \\
\hline $\mathrm{H}$ & -5.9535 & -4.6774 & 2.8272 & 7.3373 & 2.4870 & -2.7927 \\
\hline $\mathrm{H}$ & -5.7073 & 2.7408 & 4.4989 & 0.5932 & 5.4305 & -5.0764 \\
\hline $\mathrm{H}$ & -7.2335 & 2.3098 & 3.6692 & 1.5954 & 6.7461 & -4.3935 \\
\hline $\mathrm{H}$ & -6.0339 & 1.0370 & 4.0404 & 2.2744 & 5.0980 & -4.5446 \\
\hline $\mathrm{H}$ & -0.1556 & 6.2057 & 3.7166 & -5.4858 & 3.4614 & -3.9712 \\
\hline $\mathrm{H}$ & -1.5982 & 7.1115 & 3.1534 & -5.2905 & 5.1833 & -3.5200 \\
\hline $\mathrm{H}$ & -1.7666 & 5.4229 & 3.7269 & -3.8722 & 4.2402 & -4.0701 \\
\hline $\mathrm{H}$ & 5.4081 & 6.3226 & 1.7630 & -8.5029 & -1.2154 & -1.6409 \\
\hline $\mathrm{H}$ & 4.8270 & 7.4456 & 0.4968 & -9.0594 & 0.0014 & -0.4528 \\
\hline $\mathrm{H}$ & 3.6904 & 6.8457 & 1.7401 & -7.9645 & 0.4929 & -1.7789 \\
\hline $\mathrm{H}$ & 5.4159 & -0.2383 & 3.8007 & -2.9305 & -4.7492 & -3.7009 \\
\hline $\mathrm{H}$ & 6.6193 & 0.9637 & 3.2228 & -4.4492 & -5.3887 & -2.9834 \\
\hline $\mathrm{H}$ & 4.8852 & 1.3951 & 3.2950 & -4.2303 & -3.6259 & -3.1964 \\
\hline $\mathrm{H}$ & 4.9361 & -6.2145 & 2.9181 & 2.7007 & -7.1271 & -2.8875 \\
\hline $\mathrm{H}$ & 6.2684 & -5.9637 & 1.7496 & 2.0258 & -8.1964 & -1.6207 \\
\hline $\mathrm{H}$ & 5.6628 & -4.5843 & 2.7127 & 0.9328 & -7.1489 & -2.5779 \\
\hline $\mathrm{H}$ & -1.7853 & -6.3430 & 3.7993 & 6.9262 & -2.0234 & -3.3911 \\
\hline $\mathrm{H}$ & -1.1550 & -7.6791 & 2.7894 & 7.5982 & -3.1820 & -2.2040 \\
\hline $\mathrm{H}$ & -0.0345 & -6.4317 & 3.4153 & 6.0195 & -3.5162 & -2.9823 \\
\hline $\mathrm{C}$ & 0.7730 & 3.0650 & 2.9151 & 1.4436 & 1.2867 & -3.0612 \\
\hline $\mathrm{C}$ & 1.8475 & -2.1601 & 2.7715 & -1.2247 & -2.8188 & -5.2666 \\
\hline $\mathrm{C}$ & 2.7414 & 2.6710 & 4.8517 & 3.0006 & -1.0060 & -2.7492 \\
\hline $\mathrm{C}$ & 1.7563 & 4.0372 & 3.1286 & 2.8367 & 1.3639 & -3.1726 \\
\hline $\mathrm{C}$ & 3.2165 & -1.4740 & 5.0757 & 0.4646 & -4.3030 & -3.6628 \\
\hline $\mathrm{C}$ & 2.9617 & -2.9052 & 3.1572 & -0.9363 & -4.1387 & -5.6124 \\
\hline $\mathrm{C}$ & 2.7541 & 3.8380 & 4.0820 & 3.6175 & 0.2146 & -3.0409 \\
\hline $\mathrm{C}$ & 3.6554 & -2.5584 & 4.3168 & -0.0994 & -4.8968 & -4.7909 \\
\hline $\mathrm{C}$ & -3.4022 & -1.8863 & 3.5282 & -4.8190 & 0.2189 & -3.1654 \\
\hline $\mathrm{C}$ & -1.5432 & 1.3411 & 3.0364 & -1.3013 & 1.1423 & -2.3197 \\
\hline $\mathrm{C}$ & -1.0632 & -1.4033 & 3.5069 & -2.7465 & -0.9712 & -3.5035 \\
\hline $\mathrm{C}$ & -2.6382 & 0.3970 & 3.5215 & -2.7708 & 0.8716 & -2.0359 \\
\hline $\mathrm{C}$ & 1.6819 & 0.4684 & 5.5281 & 0.9343 & -2.3597 & -2.1522 \\
\hline $\mathrm{C}$ & 0.7638 & 1.8860 & 3.6766 & 0.8199 & 0.0594 & -2.7843 \\
\hline $\mathrm{C}$ & 1.3856 & -1.0789 & 3.5357 & -0.7025 & -2.2228 & -4.1074 \\
\hline $\mathrm{C}$ & 1.7469 & 1.7096 & 4.6764 & 1.6183 & -1.0832 & -2.5732 \\
\hline $\mathrm{C}$ & 2.0834 & -0.7372 & 4.7083 & 0.1852 & -2.9746 & -3.3152 \\
\hline $\mathrm{C}$ & 0.0732 & -0.4480 & 3.0824 & -1.2342 & -0.8217 & -3.7866 \\
\hline
\end{tabular}




\begin{tabular}{|c|c|c|c|c|c|c|c|c|c|}
\hline & & & & & & & & & \\
\hline $\mathrm{N}$ & -2.3778 & -0.9626 & 3.0458 & -3.3665 & 0.3182 & -3.2446 & & & \\
\hline $\mathrm{N}$ & -0.2542 & 0.9071 & 3.5764 & -0.5905 & -0.0975 & -2.6592 & & & \\
\hline $\mathrm{H}$ & 0.0100 & 3.2469 & 2.1678 & 0.8570 & 2.1905 & -3.1847 & & & \\
\hline $\mathrm{H}$ & 1.3165 & -2.4260 & 1.8607 & -1.8801 & -2.2343 & -5.9073 & & & \\
\hline $\mathrm{H}$ & 3.4931 & 2.5119 & 5.6209 & 3.6003 & -1.9018 & -2.6357 & & & \\
\hline $\mathrm{H}$ & 1.7413 & 4.9502 & 2.5462 & 3.2966 & 2.3288 & -3.3590 & & & \\
\hline $\mathrm{H}$ & 3.7546 & -1.1841 & 5.9746 & 1.1428 & -4.8640 & -3.0296 & & & \\
\hline $\mathrm{H}$ & 3.2841 & -3.7435 & 2.5565 & -1.3628 & -4.5675 & -6.5145 & & & \\
\hline $\mathrm{H}$ & 3.5253 & 4.5871 & 4.2341 & 4.6996 & 0.2695 & -3.1165 & & & \\
\hline $\mathrm{H}$ & 4.5302 & -3.1245 & 4.6230 & 0.1317 & -5.9293 & -5.0368 & & & \\
\hline $\mathrm{H}$ & -4.3850 & -1.5448 & 3.1968 & -5.2389 & 1.1981 & -2.9201 & & & \\
\hline $\mathrm{H}$ & -3.2229 & -2.8904 & 3.1281 & -5.2133 & -0.1045 & -4.1363 & & & \\
\hline $\mathrm{H}$ & -3.4086 & -1.9511 & 4.6343 & -5.1548 & -0.5013 & -2.4058 & & & \\
\hline $\mathrm{H}$ & -1.7579 & 2.3569 & 3.3753 & -0.8104 & 1.5780 & -1.4432 & & & \\
\hline $\mathrm{H}$ & -1.5408 & 1.3476 & 1.9314 & -1.2572 & 1.8848 & -3.1349 & & & \\
\hline $\mathrm{H}$ & -0.8637 & -2.3969 & 3.0912 & -3.2205 & -1.4242 & -4.3781 & & & \\
\hline $\mathrm{H}$ & -1.0328 & -1.4854 & 4.6120 & -2.8764 & -1.6694 & -2.6546 & & & \\
\hline $\mathrm{H}$ & -2.6678 & 0.4328 & 4.6285 & -2.8648 & 0.1758 & -1.1774 & & & \\
\hline $\mathrm{H}$ & -3.6077 & 0.7355 & 3.1454 & -3.2793 & 1.7997 & -1.7776 & & & \\
\hline $\mathrm{H}$ & 2.3444 & 0.5649 & 6.3934 & 1.6720 & -3.0736 & -1.7768 & & & \\
\hline $\mathrm{H}$ & 0.6517 & 0.3451 & 5.8872 & 0.2344 & -2.1278 & -1.3387 & & & \\
\hline $\mathrm{H}$ & 0.0825 & -0.4250 & 1.9774 & -1.1428 & -0.2065 & -4.6998 & & & \\
\hline & & CR2 & & & NR2 & & & M2 & \\
\hline atom & $\mathbf{x}$ & $\mathbf{y}$ & $\mathbf{z}$ & $\mathbf{x}$ & $\mathbf{y}$ & $\mathbf{z}$ & $\mathbf{x}$ & $\mathbf{y}$ & $\mathbf{Z}$ \\
\hline $\mathrm{O}$ & 3.5051 & -3.9089 & 0.3804 & -4.6083 & 1.8644 & -2.6496 & 4.3814 & 3.0220 & -2.9908 \\
\hline $\mathrm{C}$ & 2.0722 & -3.8504 & 0.2294 & -4.1947 & 0.5822 & -2.1318 & 3.3178 & 3.2834 & -2.0519 \\
\hline $\mathrm{C}$ & 1.5976 & -5.1571 & -0.4004 & -5.4493 & -0.2257 & -1.7951 & 3.7372 & 4.4507 & -1.1534 \\
\hline $\mathrm{C}$ & 2.3066 & -5.4335 & -1.7334 & -6.3886 & 0.5201 & -0.8400 & 5.0904 & 4.2108 & -0.4734 \\
\hline $\mathrm{C}$ & 3.8222 & -5.3093 & -1.5853 & -6.6805 & 1.9217 & -1.3661 & 6.1389 & 3.8079 & -1.5061 \\
\hline $\mathrm{C}$ & 4.1880 & -4.0105 & -0.8477 & -5.3665 & 2.6274 & -1.7392 & 5.6045 & 2.6692 & -2.3894 \\
\hline $\mathrm{H}$ & 1.8110 & -3.0268 & -0.4446 & -3.6110 & 0.7296 & -1.2162 & 3.1500 & 2.3952 & -1.4320 \\
\hline $\mathrm{H}$ & 1.7883 & -5.9883 & 0.2914 & -5.9903 & -0.4446 & -2.7256 & 3.8102 & 5.3600 & -1.7653 \\
\hline $\mathrm{H}$ & 1.9786 & -4.6574 & -2.4402 & -5.8647 & 0.6265 & 0.1209 & 4.9661 & 3.3699 & 0.2250 \\
\hline $\mathrm{H}$ & 4.1983 & -6.1594 & -0.9990 & -7.3071 & 1.8493 & -2.2661 & 6.3607 & 4.6707 & -2.1496 \\
\hline $\mathrm{H}$ & 5.2554 & -4.0050 & -0.6076 & -5.5825 & 3.5753 & -2.2409 & 6.3027 & 2.4767 & -3.2096 \\
\hline $\mathrm{C}$ & 1.4727 & -3.5789 & 1.6042 & -3.3385 & -0.0960 & -3.1982 & 2.0715 & 3.6129 & -2.8635 \\
\hline $\mathrm{O}$ & 0.2007 & -5.0389 & -0.6774 & -5.0698 & -1.4347 & -1.1364 & 2.7828 & 4.6284 & -0.1034 \\
\hline $\mathrm{O}$ & 1.9802 & -6.7289 & -2.2048 & -7.6000 & -0.1992 & -0.6883 & 5.5138 & 5.3832 & 0.1982 \\
\hline $\mathrm{O}$ & 4.4960 & -5.2432 & -2.8375 & -7.3083 & 2.7589 & -0.4011 & 7.3388 & 3.3186 & -0.9190 \\
\hline $\mathrm{O}$ & 3.8552 & -2.9219 & -1.6814 & -4.6349 & 2.8433 & -0.5516 & 5.4492 & 1.5190 & -1.5842 \\
\hline $\mathrm{H}$ & 0.4620 & -4.0052 & 1.6636 & -3.3867 & -1.1848 & -3.0780 & 1.3362 & 4.1116 & -2.2211 \\
\hline $\mathrm{O}$ & 1.4509 & -2.1855 & 1.8919 & -1.9814 & 0.3431 & -3.1852 & 1.4951 & 2.4747 & -3.4948 \\
\hline $\mathrm{H}$ & 2.0956 & -4.0562 & 2.3675 & -3.7454 & 0.1637 & -4.1814 & 2.3609 & 4.3030 & -3.6643 \\
\hline $\mathrm{H}$ & 1.0235 & -6.7192 & -2.4219 & -7.3764 & -1.0549 & -0.2652 & 4.8227 & 5.6108 & 0.8551 \\
\hline $\mathrm{C}$ & 4.7682 & -6.5053 & -3.4503 & -8.7285 & 2.6237 & -0.3115 & 8.2844 & 4.3200 & -0.5364 \\
\hline $\mathrm{C}$ & 0.3202 & -1.5416 & 1.3262 & -1.2165 & -0.2925 & -2.1632 & 0.7118 & 1.6927 & -2.5986 \\
\hline $\mathrm{O}$ & 5.8394 & 0.1632 & -1.8055 & -2.5617 & 5.8100 & 0.0362 & 6.0527 & -1.9037 & -2.7646 \\
\hline $\mathrm{C}$ & 4.9205 & -0.7811 & -1.2265 & -2.9111 & 4.5449 & -0.5529 & 5.3191 & -0.6741 & -2.6183 \\
\hline $\mathrm{C}$ & 4.8892 & -2.0339 & -2.1038 & -4.3425 & 4.1838 & -0.1560 & 6.2044 & 0.3493 & -1.9034 \\
\hline $\mathrm{C}$ & 4.5437 & -1.7013 & -3.5607 & -4.5343 & 4.1989 & 1.3658 & 6.7158 & -0.1870 & -0.5599 \\
\hline $\mathrm{C}$ & 5.3985 & -0.5450 & -4.0830 & -3.9681 & 5.4796 & 1.9829 & 7.3197 & -1.5827 & -0.7233 \\
\hline $\mathrm{C}$ & 5.4280 & 0.6167 & -3.0761 & -2.5564 & 5.7664 & 1.4466 & 6.3822 & -2.4961 & -1.5269 \\
\hline $\mathrm{H}$ & 3.9129 & -0.3524 & -1.2127 & -2.2527 & 3.7643 & -0.1582 & 4.4327 & -0.8477 & -1.9980 \\
\hline $\mathrm{H}$ & 5.8646 & -2.5375 & -2.0786 & -5.0550 & 4.8853 & -0.6093 & 7.0675 & 0.6061 & -2.5317 \\
\hline $\mathrm{H}$ & 3.4943 & -1.3685 & -3.5760 & -3.9544 & 3.3558 & 1.7718 & 5.8432 & -0.2797 & 0.1052 \\
\hline $\mathrm{H}$ & 6.4294 & -0.9012 & -4.2172 & -4.6138 & 6.3240 & 1.7044 & 8.2631 & -1.4966 & -1.2797 \\
\hline
\end{tabular}




\begin{tabular}{|c|c|c|c|c|c|c|c|c|c|}
\hline $\mathrm{H}$ & 6.1676 & 1.3593 & -3.3903 & -2.2227 & 6.7527 & 1.7829 & 6.8918 & -3.4348 & -1.7651 \\
\hline $\mathrm{C}$ & 5.3835 & -1.0520 & 0.1977 & -2.7098 & 4.6705 & -2.0555 & 4.8880 & -0.2403 & -4.0112 \\
\hline $\mathrm{O}$ & 4.7362 & -2.8392 & -4.3846 & -5.9085 & 4.0852 & 1.6941 & 7.6935 & 0.6780 & -0.0106 \\
\hline $\mathrm{O}$ & 4.9075 & -0.0020 & -5.3025 & -3.8504 & 5.4008 & 3.3985 & 7.5456 & -2.2264 & 0.5258 \\
\hline $\mathrm{O}$ & 4.1390 & 1.1857 & -3.0217 & -1.6985 & 4.7560 & 1.9233 & 5.2331 & -2.7319 & -0.7485 \\
\hline $\mathrm{H}$ & 4.8263 & -1.9121 & 0.5924 & -3.2054 & 3.8297 & -2.5578 & 4.5635 & 0.8083 & -3.9795 \\
\hline $\mathrm{O}$ & 5.2184 & 0.0691 & 1.0561 & -1.3337 & 4.7054 & -2.4142 & 3.8474 & -1.0622 & -4.5286 \\
\hline $\mathrm{H}$ & 6.4534 & -1.2873 & 0.1852 & -3.1547 & 5.6108 & -2.3982 & 5.7413 & -0.3334 & -4.6918 \\
\hline $\mathrm{H}$ & 4.4244 & -3.6223 & -3.8885 & -6.3101 & 3.4410 & 1.0770 & 7.4061 & 1.5996 & -0.1715 \\
\hline $\mathrm{C}$ & 5.3058 & -0.6991 & -6.4844 & -5.0380 & 5.7211 & 4.1255 & 8.8090 & -1.9466 & 1.1319 \\
\hline $\mathrm{C}$ & 3.8566 & 0.2892 & 1.4118 & -0.7309 & 3.4182 & -2.3556 & 2.5715 & -0.6936 & -4.0154 \\
\hline $\mathrm{O}$ & 3.3941 & 4.6431 & -1.9993 & 1.9542 & 4.9988 & 2.2647 & 2.8623 & -5.5190 & -1.0866 \\
\hline $\mathrm{C}$ & 3.6616 & 3.2437 & -1.7867 & 0.7204 & 4.7326 & 1.5682 & 3.3886 & -4.2048 & -1.3586 \\
\hline $\mathrm{C}$ & 4.0034 & 2.6015 & -3.1364 & -0.4407 & 5.1214 & 2.4885 & 4.7343 & -4.0643 & -0.6417 \\
\hline $\mathrm{C}$ & 2.8916 & 2.8234 & -4.1670 & -0.3691 & 4.3729 & 3.8258 & 4.6049 & -4.3329 & 0.8626 \\
\hline $\mathrm{C}$ & 2.5441 & 4.3073 & -4.2501 & 1.0096 & 4.5419 & 4.4587 & 3.9081 & -5.6685 & 1.1034 \\
\hline $\mathrm{C}$ & 2.2932 & 4.8878 & -2.8491 & 2.1197 & 4.2365 & 3.4411 & 2.6134 & -5.7606 & 0.2809 \\
\hline $\mathrm{H}$ & 2.7713 & 2.7586 & -1.3695 & 0.6522 & 3.6624 & 1.3383 & 2.6976 & -3.4441 & -0.9758 \\
\hline $\mathrm{H}$ & 4.9314 & 3.0453 & -3.5216 & -0.4056 & 6.2017 & 2.6832 & 5.4506 & -4.7796 & -1.0679 \\
\hline $\mathrm{H}$ & 1.9978 & 2.2858 & -3.8151 & -0.5127 & 3.3025 & 3.6118 & 3.9694 & -3.5403 & 1.2864 \\
\hline $\mathrm{H}$ & 3.3977 & 4.8464 & -4.6832 & 1.1271 & 5.5882 & 4.7728 & 4.5708 & -6.4792 & 0.7711 \\
\hline $\mathrm{H}$ & 2.2028 & 5.9758 & -2.9171 & 3.0856 & 4.5375 & 3.8575 & 2.2188 & -6.7794 & 0.3379 \\
\hline $\mathrm{C}$ & 4.8085 & 3.1420 & -0.8038 & 0.7528 & 5.5239 & 0.2760 & 3.5022 & -4.0728 & -2.8628 \\
\hline $\mathrm{O}$ & 3.3015 & 2.3664 & -5.4429 & -1.3564 & 4.8590 & 4.7168 & 5.8827 & -4.3525 & 1.4718 \\
\hline $\mathrm{O}$ & 1.3718 & 4.5428 & -5.0258 & 1.2075 & 3.6717 & 5.5696 & 3.5535 & -5.8553 & 2.4712 \\
\hline $\mathrm{O}$ & 1.1119 & 4.3207 & -2.3323 & 2.1047 & 2.8549 & 3.1677 & 1.6881 & -4.8376 & 0.8045 \\
\hline $\mathrm{H}$ & 5.1128 & 2.0926 & -0.7063 & -0.2276 & 5.4832 & -0.2135 & 4.1010 & -3.1927 & -3.1245 \\
\hline $\mathrm{O}$ & 4.3949 & 3.6578 & 0.4525 & 1.7541 & 4.9687 & -0.5634 & 2.1936 & -3.9589 & -3.4049 \\
\hline $\mathrm{H}$ & 5.6667 & 3.7180 & -1.1902 & 0.9840 & 6.5778 & 0.5060 & 4.0078 & -4.9698 & -3.2582 \\
\hline $\mathrm{H}$ & 3.6937 & 1.4774 & -5.3293 & -2.2082 & 4.8759 & 4.2359 & 6.3520 & -3.5371 & 1.2021 \\
\hline $\mathrm{C}$ & 1.6125 & 4.7565 & -6.4204 & 0.8041 & 4.2126 & 6.8308 & 4.5563 & -6.4966 & 3.2648 \\
\hline $\mathrm{C}$ & 5.4268 & 3.5274 & 1.4204 & 1.9107 & 5.7018 & -1.7724 & 2.2093 & -4.0070 & -4.8240 \\
\hline $\mathrm{O}$ & -1.3057 & 5.3430 & 0.2248 & 4.6923 & 0.6689 & 1.7604 & -1.9629 & -4.6414 & 0.5619 \\
\hline $\mathrm{C}$ & -0.2021 & 4.6057 & -0.3394 & 3.4796 & 1.4492 & 1.7627 & -0.5984 & -4.2745 & 0.2665 \\
\hline $\mathrm{C}$ & 0.1784 & 5.1987 & -1.6990 & 3.3649 & 2.1849 & 3.0971 & 0.3357 & -5.2753 & 0.9492 \\
\hline $\mathrm{C}$ & -1.0159 & 5.3535 & -2.6433 & 3.4309 & 1.2251 & 4.2862 & 0.0678 & -5.3944 & 2.4523 \\
\hline $\mathrm{C}$ & -2.1382 & 6.0981 & -1.9284 & 4.6806 & 0.3562 & 4.1701 & -1.4129 & -5.6679 & 2.6969 \\
\hline $\mathrm{C}$ & -2.4545 & 5.4069 & -0.5905 & 4.7523 & -0.3040 & 2.7793 & -2.2729 & -4.6443 & 1.9340 \\
\hline $\mathrm{H}$ & -0.4961 & 3.5587 & -0.4789 & 2.6163 & 0.7830 & 1.6494 & -0.3977 & -3.2752 & 0.6677 \\
\hline $\mathrm{H}$ & 0.6268 & 6.1896 & -1.5427 & 4.1899 & 2.9054 & 3.1789 & 0.1975 & -6.2623 & 0.4875 \\
\hline $\mathrm{H}$ & -1.3825 & 4.3476 & -2.8928 & 2.5525 & 0.5647 & 4.2356 & 0.3109 & -4.4253 & 2.9120 \\
\hline $\mathrm{H}$ & -1.8128 & 7.1254 & -1.7126 & 5.5693 & 0.9928 & 4.2835 & -1.6621 & -6.6731 & 2.3289 \\
\hline $\mathrm{H}$ & -3.1852 & 5.9995 & -0.0319 & 5.7186 & -0.8037 & 2.6610 & -3.3286 & -4.9214 & 2.0123 \\
\hline $\mathrm{C}$ & 0.9644 & 4.7160 & 0.6410 & 3.5587 & 2.4144 & 0.5900 & -0.4204 & -4.2880 & -1.2503 \\
\hline $\mathrm{O}$ & -0.6274 & 6.0629 & -3.8063 & 3.4603 & 1.9514 & 5.5022 & 0.8514 & -6.4375 & 3.0052 \\
\hline $\mathrm{O}$ & -3.3501 & 6.1217 & -2.6697 & 4.7140 & -0.6936 & 5.1268 & -1.7845 & -5.5474 & 4.0648 \\
\hline $\mathrm{O}$ & -2.9625 & 4.1175 & -0.8642 & 3.6944 & -1.2300 & 2.6625 & -2.0523 & -3.3598 & 2.4859 \\
\hline $\mathrm{H}$ & 1.9123 & 4.6076 & 0.0980 & 2.9270 & 3.2888 & 0.7737 & 0.6336 & -4.4598 & -1.4958 \\
\hline $\mathrm{O}$ & 0.8978 & 3.7731 & 1.7036 & 3.2093 & 1.7981 & -0.6400 & -0.8898 & -3.1070 & -1.8886 \\
\hline $\mathrm{H}$ & 0.9320 & 5.7129 & 1.0935 & 4.5971 & 2.7501 & 0.4905 & -1.0102 & -5.1185 & -1.6536 \\
\hline $\mathrm{H}$ & 0.0348 & 5.5091 & -4.2712 & 2.6419 & 2.4891 & 5.5378 & 1.7915 & -6.1949 & 2.8706 \\
\hline $\mathrm{C}$ & -3.4692 & 7.1854 & -3.6170 & 5.2828 & -0.3507 & 6.3923 & -1.5571 & -6.7094 & 4.8670 \\
\hline $\mathrm{C}$ & 1.3811 & 2.4955 & 1.3032 & 1.8062 & 1.6845 & -0.8364 & -0.0052 & -2.0026 & -1.7334 \\
\hline $\mathrm{O}$ & -5.3835 & 2.1960 & 1.1244 & 3.5781 & -4.2882 & 0.6132 & -4.6232 & -0.8236 & 3.1325 \\
\hline $\mathrm{C}$ & -4.0881 & 2.7014 & 0.7470 & 3.4862 & -2.8715 & 0.8712 & -3.7357 & -1.6458 & 2.3519 \\
\hline $\mathrm{C}$ & -4.2497 & 3.7513 & -0.3564 & 3.9907 & -2.5807 & 2.2929 & -3.0991 & -2.7262 & 3.2300 \\
\hline
\end{tabular}




\begin{tabular}{|c|c|c|c|c|c|c|c|c|c|}
\hline $\mathrm{C}$ & -5.0429 & 3.2001 & -1.5487 & 3.3244 & -3.4629 & 3.3569 & -2.4203 & -2.1174 & 4.4653 \\
\hline $\mathrm{C}$ & -6.3421 & 2.5502 & -1.0725 & 3.3778 & -4.9304 & 2.9412 & -3.3493 & -1.1288 & 5.1722 \\
\hline $\mathrm{C}$ & -6.0724 & 1.5628 & 0.0729 & 2.8524 & -5.0933 & 1.5090 & -3.9650 & -0.1388 & 4.1754 \\
\hline $\mathrm{H}$ & -3.4774 & 1.8812 & 0.3523 & 2.4415 & -2.5499 & 0.7862 & -2.9159 & -1.0286 & 1.9665 \\
\hline $\mathrm{H}$ & -4.7736 & 4.6322 & 0.0381 & 5.0743 & -2.7581 & 2.3326 & -3.8448 & -3.4626 & 3.5547 \\
\hline $\mathrm{H}$ & -4.4273 & 2.4152 & -2.0151 & 2.2654 & -3.1692 & 3.4148 & -1.5512 & -1.5472 & 4.1017 \\
\hline $\mathrm{H}$ & -7.0135 & 3.3349 & -0.6971 & 4.4202 & -5.2764 & 2.9722 & -4.1719 & -1.6866 & 5.6396 \\
\hline $\mathrm{H}$ & -7.0208 & 1.2163 & 0.4945 & 2.9944 & -6.1257 & 1.1747 & -4.7317 & 0.4601 & 4.6761 \\
\hline $\mathrm{C}$ & -3.4469 & 3.2589 & 2.0093 & 4.3510 & -2.1762 & -0.1749 & -4.5458 & -2.1683 & 1.1619 \\
\hline $\mathrm{O}$ & -5.3576 & 4.2276 & -2.4679 & 3.9738 & -3.3140 & 4.6050 & -2.0291 & -3.1175 & 5.3878 \\
\hline $\mathrm{O}$ & -6.9925 & 1.8043 & -2.0936 & 2.5655 & -5.7679 & 3.7557 & -2.6590 & -0.3560 & 6.1515 \\
\hline $\mathrm{O}$ & -5.3261 & 0.4772 & -0.4371 & 1.4806 & -4.7491 & 1.4947 & -2.9345 & 0.6803 & 3.6786 \\
\hline $\mathrm{H}$ & -2.6324 & 3.9425 & 1.7351 & 4.4707 & -1.1217 & 0.1033 & -4.3190 & -3.2247 & 0.9671 \\
\hline $\mathrm{O}$ & -2.9627 & 2.2219 & 2.8528 & 3.8362 & -2.2968 & -1.4912 & -4.2936 & -1.3999 & -0.0072 \\
\hline $\mathrm{H}$ & -4.2039 & 3.8136 & 2.5741 & 5.3401 & -2.6487 & -0.1715 & -5.6112 & -2.0792 & 1.3941 \\
\hline $\mathrm{H}$ & -4.5476 & 4.7571 & -2.6196 & 4.0667 & -2.3557 & 4.7846 & -1.7400 & -3.9027 & 4.8793 \\
\hline $\mathrm{C}$ & -7.8394 & 2.5570 & -2.9646 & 3.1658 & -6.2142 & 4.9739 & -2.6579 & -0.9191 & 7.4657 \\
\hline $\mathrm{C}$ & -1.6700 & 1.7847 & 2.4505 & 2.8972 & -1.2726 & -1.8077 & -3.0980 & -1.8416 & -0.6514 \\
\hline $\mathrm{O}$ & -5.6205 & -2.9816 & 0.7653 & -1.0928 & -6.4245 & -0.5165 & -3.1289 & 3.9359 & 2.0164 \\
\hline $\mathrm{C}$ & -5.1358 & -1.6257 & 0.7816 & 0.0149 & -5.5439 & -0.2539 & -2.9371 & 2.5100 & 2.0858 \\
\hline $\mathrm{C}$ & -5.8605 & -0.8418 & -0.3162 & 0.5186 & -5.7575 & 1.1754 & -3.2065 & 2.0730 & 3.5238 \\
\hline $\mathrm{C}$ & -5.6621 & -1.4990 & -1.6888 & -0.6127 & -5.5978 & 2.1990 & -2.2632 & 2.7971 & 4.4946 \\
\hline $\mathrm{C}$ & -5.9985 & -2.9891 & -1.6284 & -1.8331 & -6.4235 & 1.7915 & -2.3217 & 4.3093 & 4.2802 \\
\hline $\mathrm{C}$ & -5.3140 & -3.6641 & -0.4308 & -2.2002 & -6.1775 & 0.3216 & -2.1876 & 4.6528 & 2.7852 \\
\hline $\mathrm{H}$ & -4.0615 & -1.6169 & 0.5637 & -0.3198 & -4.5036 & -0.3331 & -1.8941 & 2.2710 & 1.8529 \\
\hline $\mathrm{H}$ & -6.9355 & -0.8037 & -0.0953 & 0.9561 & -6.7587 & 1.2838 & -4.2475 & 2.2993 & 3.7891 \\
\hline $\mathrm{H}$ & -4.5944 & -1.4071 & -1.9424 & -0.9116 & -4.5381 & 2.1864 & -1.2402 & 2.4666 & 4.2593 \\
\hline $\mathrm{H}$ & -7.0836 & -3.0979 & -1.4961 & -1.5925 & -7.4895 & 1.9012 & -3.2986 & 4.6787 & 4.6228 \\
\hline $\mathrm{H}$ & -5.7024 & -4.6797 & -0.3076 & -2.9783 & -6.8821 & 0.0123 & -2.4092 & 5.7126 & 2.6279 \\
\hline $\mathrm{C}$ & -5.3743 & -1.0868 & 2.1834 & 1.0674 & -5.8200 & -1.3175 & -3.8417 & 1.8691 & 1.0460 \\
\hline $\mathrm{O}$ & -6.4804 & -0.8874 & -2.6683 & -0.1981 & -6.0009 & 3.4909 & -2.6068 & 2.4881 & 5.8337 \\
\hline $\mathrm{O}$ & -5.5761 & -3.6836 & -2.7972 & -2.9812 & -6.0973 & 2.5696 & -1.2756 & 5.0034 & 4.9485 \\
\hline $\mathrm{O}$ & -3.9267 & -3.6838 & -0.6693 & -2.6491 & -4.8490 & 0.1998 & -0.8719 & 4.3509 & 2.3816 \\
\hline $\mathrm{H}$ & -5.2188 & 0.0005 & 2.1850 & 2.0410 & -5.4467 & -0.9725 & -3.9346 & 0.7973 & 1.2513 \\
\hline $\mathrm{O}$ & -4.5315 & -1.7193 & 3.1419 & 0.7359 & -5.2254 & -2.5663 & -3.3703 & 2.0671 & -0.2857 \\
\hline $\mathrm{H}$ & -6.4085 & -1.3020 & 2.4747 & 1.1329 & -6.9015 & -1.4780 & -4.8347 & 2.3272 & 1.0939 \\
\hline $\mathrm{H}$ & -6.4506 & 0.0808 & -2.5276 & 0.7272 & -5.7068 & 3.6181 & -2.5455 & 1.5156 & 5.9318 \\
\hline $\mathrm{C}$ & -6.5395 & -3.7328 & -3.8517 & -3.1464 & -6.8802 & 3.7541 & -1.5489 & 5.3406 & 6.3100 \\
\hline $\mathrm{C}$ & -3.2323 & -1.1396 & 3.1851 & 1.0326 & -3.8310 & -2.5766 & -2.1134 & 1.4413 & -0.5354 \\
\hline $\mathrm{O}$ & -1.3682 & -5.8581 & 0.8357 & -4.6905 & -3.3211 & -2.4397 & 0.8524 & 5.8702 & -0.4926 \\
\hline $\mathrm{C}$ & -2.2290 & -4.7060 & 0.7453 & -3.4675 & -3.6667 & -1.7592 & -0.0101 & 4.9199 & 0.1621 \\
\hline $\mathrm{C}$ & -3.2077 & -4.8916 & -0.4222 & -3.8080 & -4.5973 & -0.5933 & -0.1409 & 5.3222 & 1.6348 \\
\hline $\mathrm{C}$ & -2.5066 & -5.2389 & -1.7379 & -4.8385 & -3.9705 & 0.3533 & 1.2296 & 5.4049 & 2.3146 \\
\hline $\mathrm{C}$ & -1.5605 & -6.4132 & -1.5222 & -6.0546 & -3.4967 & -0.4346 & 2.1684 & 6.2939 & 1.5045 \\
\hline $\mathrm{C}$ & -0.6272 & -6.1259 & -0.3354 & -5.6178 & -2.6342 & -1.6293 & 2.1670 & 5.8885 & 0.0209 \\
\hline $\mathrm{H}$ & -1.6265 & -3.8056 & 0.5752 & -2.9928 & -2.7588 & -1.3678 & 0.4318 & 3.9175 & 0.1001 \\
\hline $\mathrm{H}$ & -3.9010 & -5.7086 & -0.1793 & -4.2177 & -5.5362 & -0.9893 & -0.6281 & 6.3044 & 1.7009 \\
\hline $\mathrm{H}$ & -1.9035 & -4.3698 & -2.0399 & -4.3713 & -3.0879 & 0.8154 & 1.6542 & 4.3892 & 2.3282 \\
\hline $\mathrm{H}$ & -2.1463 & -7.3097 & -1.2781 & -6.5848 & -4.3724 & -0.8334 & 1.8093 & 7.3304 & 1.5624 \\
\hline $\mathrm{H}$ & -0.0288 & -7.0148 & -0.1152 & -6.4833 & -2.4320 & -2.2672 & 2.7048 & 6.6425 & -0.5616 \\
\hline $\mathrm{C}$ & -2.9799 & -4.6032 & 2.0567 & -2.5623 & -4.3106 & -2.7879 & -1.3318 & 4.9294 & -0.5748 \\
\hline $\mathrm{O}$ & -3.4621 & -5.5750 & -2.7269 & -5.2445 & -4.9116 & 1.3299 & 1.1025 & 5.9297 & 3.6235 \\
\hline $\mathrm{O}$ & -0.7434 & -6.6593 & -2.6642 & -6.9390 & -2.7079 & 0.3575 & 3.5126 & 6.2151 & 1.9684 \\
\hline $\mathrm{H}$ & -3.7764 & -3.8543 & 1.9730 & -1.7225 & -4.8217 & -2.3036 & -2.0747 & 4.3514 & -0.0120 \\
\hline $\mathrm{O}$ & -2.0903 & -4.2559 & 3.1086 & -2.0915 & -3.2948 & -3.6665 & -1.1311 & 4.3590 & -1.8589 \\
\hline $\mathrm{H}$ & -3.4445 & -5.5828 & 2.2603 & -3.1484 & -5.0597 & -3.3450 & -1.6904 & 5.9685 & -0.6637 \\
\hline
\end{tabular}




\begin{tabular}{|c|c|c|c|c|c|c|c|c|c|}
\hline $\mathrm{H}$ & -4.1096 & -4.8420 & -2.7736 & -4.4382 & -5.2297 & 1.7859 & 0.3705 & 5.4538 & 4.0645 \\
\hline $\mathrm{C}$ & -1.2931 & -7.5913 & -3.6014 & -7.9562 & -3.4521 & 1.0349 & 3.8588 & 7.1792 & 2.9668 \\
\hline $\mathrm{C}$ & -2.7515 & -4.3340 & 4.3665 & -1.5473 & -3.8377 & -4.8590 & -2.3385 & 4.3095 & -2.6048 \\
\hline $\mathrm{H}$ & 0.4081 & -0.4745 & 1.5445 & -0.1952 & 0.0836 & -2.2481 & 0.2397 & 0.9038 & -3.1911 \\
\hline $\mathrm{H}$ & 0.2704 & -1.6753 & 0.2353 & -1.6024 & -0.0584 & -1.1604 & 1.3269 & 1.2179 & -1.8193 \\
\hline $\mathrm{H}$ & -0.6089 & -1.9321 & 1.7684 & -1.2164 & -1.3811 & -2.3089 & -0.0574 & 2.3112 & -2.1248 \\
\hline $\mathrm{H}$ & 3.8509 & 1.0560 & 2.1885 & 0.2958 & 3.5362 & -2.7020 & 1.8328 & -1.3465 & -4.4839 \\
\hline $\mathrm{H}$ & 3.2707 & 0.6469 & 0.5547 & -0.7029 & 3.0260 & -1.3304 & 2.5133 & -0.8344 & -2.9275 \\
\hline $\mathrm{H}$ & 3.3853 & -0.6299 & 1.7886 & -1.2666 & 2.6913 & -2.9826 & 2.3508 & 0.3578 & -4.2401 \\
\hline $\mathrm{H}$ & 5.0509 & 3.9484 & 2.3561 & 2.7199 & 5.2242 & -2.3337 & 1.1802 & -3.8577 & -5.1632 \\
\hline $\mathrm{H}$ & 5.6938 & 2.4721 & 1.5683 & 0.9809 & 5.6945 & -2.3589 & 2.8521 & -3.2188 & -5.2390 \\
\hline $\mathrm{H}$ & 6.3272 & 4.0827 & 1.1138 & 2.1825 & 6.7487 & -1.5610 & 2.5707 & -4.9844 & -5.1810 \\
\hline $\mathrm{H}$ & 1.3671 & 1.8529 & 2.1863 & 1.6632 & 1.3247 & -1.8594 & -0.4921 & -1.1459 & -2.2031 \\
\hline $\mathrm{H}$ & 0.7461 & 2.0429 & 0.5266 & 1.3527 & 0.9608 & -0.1435 & 0.1700 & -1.7629 & -0.6745 \\
\hline $\mathrm{H}$ & 2.4068 & 2.5828 & 0.9336 & 1.3297 & 2.6615 & -0.7175 & 0.9540 & -2.2129 & -2.2213 \\
\hline $\mathrm{H}$ & -1.3728 & 0.9973 & 3.1415 & 2.5894 & -1.4226 & -2.8443 & -2.9085 & -1.1832 & -1.4992 \\
\hline $\mathrm{H}$ & -1.6786 & 1.3734 & 1.4294 & 2.0077 & -1.3254 & -1.1604 & -2.2407 & -1.8133 & 0.0333 \\
\hline $\mathrm{H}$ & -0.9526 & 2.6068 & 2.5105 & 3.3612 & -0.2888 & -1.6982 & -3.2052 & -2.8764 & -0.9913 \\
\hline $\mathrm{H}$ & -2.6601 & -1.6761 & 3.9436 & 0.8951 & -3.4727 & -3.5954 & -2.0531 & 1.2329 & -1.6058 \\
\hline $\mathrm{H}$ & -2.7082 & -1.2379 & 2.2232 & 0.3598 & -3.2618 & -1.9191 & -1.2807 & 2.0952 & -0.2484 \\
\hline $\mathrm{H}$ & -3.2983 & -0.0741 & 3.4367 & 2.0671 & -3.6567 & -2.2588 & -2.0302 & 0.4923 & 0.0076 \\
\hline $\mathrm{H}$ & -2.0410 & -4.0047 & 5.1284 & -1.1978 & -2.9981 & -5.4651 & -2.1187 & 3.7862 & -3.5393 \\
\hline $\mathrm{H}$ & -3.6370 & -3.6841 & 4.3847 & -0.7080 & -4.5138 & -4.6443 & -3.1139 & 3.7636 & -2.0531 \\
\hline $\mathrm{H}$ & -3.0640 & -5.3682 & 4.5798 & -2.3119 & -4.3961 & -5.4216 & -2.7049 & 5.3226 & -2.8336 \\
\hline $\mathrm{H}$ & 5.3703 & -6.2934 & -4.3376 & -9.0666 & 3.3873 & 0.3935 & 9.1764 & 3.7872 & -0.1973 \\
\hline $\mathrm{H}$ & 5.3407 & -7.1467 & -2.7656 & -9.1910 & 2.8019 & -1.2923 & 8.5415 & 4.9503 & -1.3991 \\
\hline $\mathrm{H}$ & 3.8417 & -7.0146 & -3.7299 & -9.0063 & 1.6269 & 0.0424 & 7.8873 & 4.9493 & 0.2649 \\
\hline $\mathrm{H}$ & 4.9597 & -0.0980 & -7.3291 & -4.7616 & 5.7268 & 5.1829 & 8.8732 & -2.5796 & 2.0204 \\
\hline $\mathrm{H}$ & 6.4003 & -0.7899 & -6.5254 & -5.4063 & 6.7164 & 3.8394 & 9.6294 & -2.1982 & 0.4450 \\
\hline $\mathrm{H}$ & 4.8609 & -1.6978 & -6.5214 & -5.8224 & 4.9811 & 3.9418 & 8.8828 & -0.8916 & 1.4114 \\
\hline $\mathrm{H}$ & 0.6446 & 4.9957 & -6.8676 & 1.0841 & 3.4754 & 7.5874 & 4.1213 & -6.6394 & 4.2569 \\
\hline $\mathrm{H}$ & 2.3024 & 5.5989 & -6.5688 & 1.3269 & 5.1583 & 7.0313 & 4.8210 & -7.4741 & 2.8376 \\
\hline $\mathrm{H}$ & 2.0317 & 3.8568 & -6.8790 & -0.2760 & 4.3836 & 6.8485 & 5.4522 & -5.8727 & 3.3287 \\
\hline $\mathrm{H}$ & -4.4814 & 7.1230 & -4.0244 & 5.3290 & -1.2766 & 6.9709 & -1.9746 & -6.4880 & 5.8524 \\
\hline $\mathrm{H}$ & -3.3358 & 8.1557 & -3.1187 & 6.2984 & 0.0476 & 6.2595 & -2.0771 & -7.5756 & 4.4353 \\
\hline $\mathrm{H}$ & -2.7289 & 7.0818 & -4.4148 & 4.6665 & 0.3909 & 6.9081 & -0.4889 & -6.9292 & 4.9441 \\
\hline $\mathrm{H}$ & -8.3555 & 1.8290 & -3.5957 & 2.4680 & -6.9306 & 5.4149 & -2.1624 & -0.1921 & 8.1140 \\
\hline $\mathrm{H}$ & -8.5788 & 3.1234 & -2.3816 & 4.1210 & -6.7155 & 4.7655 & -3.6863 & -1.0808 & 7.8184 \\
\hline $\mathrm{H}$ & -7.2568 & 3.2494 & -3.5785 & 3.3379 & -5.3781 & 5.6569 & -2.1162 & -1.8693 & 7.4784 \\
\hline $\mathrm{H}$ & -6.1091 & -4.3610 & -4.6355 & -4.0950 & -6.5713 & 4.2002 & -0.7022 & 5.9380 & 6.6573 \\
\hline $\mathrm{H}$ & -7.4770 & -4.1836 & -3.4968 & -3.1909 & -7.9503 & 3.5070 & -2.4692 & 5.9377 & 6.3803 \\
\hline $\mathrm{H}$ & -6.7431 & -2.7300 & -4.2382 & -2.3234 & -6.7029 & 4.4526 & -1.6557 & 4.4401 & 6.9218 \\
\hline $\mathrm{H}$ & -0.5344 & -7.7351 & -4.3745 & -8.5935 & -2.7195 & 1.5362 & 4.9240 & 7.0450 & 3.1701 \\
\hline $\mathrm{H}$ & -1.5037 & -8.5524 & -3.1121 & -8.5564 & -4.0284 & 0.3170 & 3.6828 & 8.1988 & 2.5966 \\
\hline $\mathrm{H}$ & -2.2130 & -7.1947 & -4.0394 & -7.5100 & -4.1321 & 1.7658 & 3.2748 & 7.0161 & 3.8771 \\
\hline $\mathrm{C}$ & 4.1134 & 2.2829 & 4.5718 & 1.3540 & 0.5166 & -4.5795 & -6.4750 & 2.6539 & -2.5014 \\
\hline $\mathrm{C}$ & 0.2087 & -1.1070 & 4.5960 & 5.9117 & 0.7102 & -2.0273 & -6.2910 & -2.5505 & -2.3914 \\
\hline $\mathrm{C}$ & 4.9817 & 0.2495 & 6.2676 & 2.8195 & -1.5898 & -5.6602 & -8.8905 & 1.8675 & -3.6562 \\
\hline $\mathrm{C}$ & 5.4428 & 1.8525 & 4.5243 & 0.7153 & -0.6883 & -4.8928 & -7.6524 & 3.3479 & -2.2047 \\
\hline $\mathrm{C}$ & 1.7029 & -2.1975 & 6.6451 & 6.5456 & -1.2061 & -3.9225 & -8.4833 & -2.2736 & -4.0555 \\
\hline $\mathrm{C}$ & 0.2172 & -2.4899 & 4.7688 & 6.8005 & -0.3119 & -1.6988 & -7.2802 & -3.5335 & -2.3922 \\
\hline $\mathrm{C}$ & 5.8817 & 0.8320 & 5.3681 & 1.4463 & -1.7436 & -5.4374 & -8.8631 & 2.9580 & -2.7792 \\
\hline $\mathrm{C}$ & 0.9603 & -3.0396 & 5.8142 & 7.1256 & -1.2754 & -2.6553 & -8.3823 & -3.4003 & -3.2389 \\
\hline $\mathrm{C}$ & -2.1129 & 3.5629 & 5.7245 & 5.3168 & 5.6306 & -4.0917 & -1.6937 & -1.1345 & -4.5536 \\
\hline C & 1.6014 & 3.5156 & 5.1732 & 2.5784 & 3.1091 & -4.5802 & -4.0877 & 1.6146 & -3.6544 \\
\hline $\mathrm{C}$ & -0.5442 & 1.7416 & 5.7010 & 5.3112 & 3.2334 & -3.8490 & -4.0001 & -1.1920 & -3.9199 \\
\hline
\end{tabular}




\begin{tabular}{|c|c|c|c|c|c|c|c|c|c|}
\hline $\mathrm{C}$ & 0.2675 & 3.9874 & 5.7417 & 3.4371 & 4.3384 & -4.8573 & -2.9602 & 0.9092 & -4.3956 \\
\hline $\mathrm{C}$ & 2.6533 & 0.0535 & 7.2754 & 4.9447 & -0.2015 & -5.5979 & -7.7107 & -0.0255 & -4.8874 \\
\hline $\mathrm{C}$ & 3.2086 & 1.7002 & 5.4711 & 2.7296 & 0.6810 & -4.8125 & -6.5007 & 1.5659 & -3.3861 \\
\hline $\mathrm{C}$ & 0.9419 & -0.2498 & 5.4238 & 5.3344 & 0.7961 & -3.3022 & -6.3699 & -1.4269 & -3.2287 \\
\hline C & 3.6573 & 0.6791 & 6.3374 & 3.4654 & -0.3913 & -5.3672 & -7.7226 & 1.1741 & -3.9718 \\
\hline $\mathrm{C}$ & 1.7108 & -0.8094 & 6.4613 & 5.6535 & -0.1809 & -4.2598 & -7.4931 & -1.2822 & -4.0653 \\
\hline C & 0.7960 & 1.2380 & 5.1348 & 4.4418 & 2.0008 & -3.5464 & -5.1638 & -0.4924 & -3.1905 \\
\hline $\mathrm{N}$ & -0.8028 & 3.1117 & 5.2797 & 4.4982 & 4.4483 & -3.8601 & -2.7552 & -0.4279 & -3.8426 \\
\hline $\mathrm{N}$ & 1.8687 & 2.1398 & 5.6094 & 3.4059 & 1.9045 & -4.6083 & -5.3352 & 0.8556 & -3.7812 \\
\hline $\mathrm{H}$ & 3.7747 & 3.0667 & 3.9036 & 0.7721 & 1.3301 & -4.1595 & -5.5417 & 2.9484 & -2.0330 \\
\hline $\mathrm{H}$ & -0.3591 & -0.6843 & 3.7751 & 5.6333 & 1.4464 & -1.2804 & -5.4444 & -2.6356 & -1.7184 \\
\hline $\mathrm{H}$ & 5.3123 & -0.5410 & 6.9367 & 3.3971 & -2.4050 & -6.0884 & -9.8267 & 1.5561 & -4.1129 \\
\hline $\mathrm{H}$ & 6.1287 & 2.3122 & 3.8201 & -0.3479 & -0.7860 & -4.6956 & -7.6191 & 4.1890 & -1.5177 \\
\hline $\mathrm{H}$ & 2.3102 & -2.6187 & 7.4424 & 6.7740 & -1.9639 & -4.6679 & -9.3511 & -2.1458 & -4.6978 \\
\hline $\mathrm{H}$ & -0.3274 & -3.1185 & 4.0739 & 7.2217 & -0.3620 & -0.6991 & -7.1919 & -4.3916 & -1.7320 \\
\hline $\mathrm{H}$ & 6.9130 & 0.4942 & 5.3318 & 0.9589 & -2.6785 & -5.6926 & -9.7783 & 3.4961 & -2.5502 \\
\hline $\mathrm{H}$ & 0.9834 & -4.1148 & 5.9679 & 7.8120 & -2.0820 & -2.4140 & -9.1635 & -4.1550 & -3.2523 \\
\hline $\mathrm{H}$ & -2.2722 & 4.5946 & 5.3907 & 4.6859 & 6.5253 & -4.0459 & -0.7669 & -0.5525 & -4.5028 \\
\hline $\mathrm{H}$ & -2.8795 & 2.9296 & 5.2690 & 6.0862 & 5.7023 & -3.3153 & -1.5224 & -2.1059 & -4.0799 \\
\hline $\mathrm{H}$ & -2.2201 & 3.5368 & 6.8267 & 5.8194 & 5.6096 & -5.0776 & -1.9417 & -1.2901 & -5.6207 \\
\hline $\mathrm{H}$ & 2.4079 & 4.1544 & 5.5443 & 1.8006 & 3.0254 & -5.3435 & -4.2443 & 2.6080 & -4.0843 \\
\hline $\mathrm{H}$ & 1.5653 & 3.5993 & 4.0751 & 2.0923 & 3.2340 & -3.5989 & -3.8101 & 1.7387 & -2.6004 \\
\hline $\mathrm{H}$ & -1.3500 & 1.1023 & 5.3276 & 6.0706 & 3.3308 & -3.0664 & -3.8325 & -2.1691 & -3.4587 \\
\hline $\mathrm{H}$ & -0.5156 & 1.6436 & 6.8048 & 5.8299 & 3.0780 & -4.8155 & -4.3054 & -1.3552 & -4.9719 \\
\hline $\mathrm{H}$ & 0.3289 & 4.0060 & 6.8481 & 3.8519 & 4.2640 & -5.8810 & -3.2073 & 0.8619 & -5.4738 \\
\hline $\mathrm{H}$ & 0.0644 & 5.0069 & 5.3946 & 2.8122 & 5.2375 & -4.8053 & -2.0323 & 1.4824 & -4.2838 \\
\hline $\mathrm{H}$ & 3.1601 & -0.5561 & 8.0293 & 5.3406 & -1.0106 & -6.2188 & -8.6573 & -0.1009 & -5.4312 \\
\hline $\mathrm{H}$ & 2.0951 & 0.8515 & 7.7804 & 5.1000 & 0.7518 & -6.1196 & -6.8963 & 0.0925 & -5.6128 \\
\hline \multirow[t]{2}{*}{$\mathrm{H}$} & 0.7192 & 1.3429 & 4.0408 & 3.9350 & 2.2082 & -2.5952 & -4.8749 & -0.3777 & -2.1340 \\
\hline & \multicolumn{3}{|c|}{ FA2 } & \multicolumn{3}{|c|}{ FV2 } & \multicolumn{3}{|c|}{$\mathbf{S}$} \\
\hline atom & $\mathbf{x}$ & $\mathbf{y}$ & $\mathbf{Z}$ & $\mathbf{x}$ & $\mathbf{y}$ & $\mathbf{z}$ & $\mathbf{x}$ & $\mathbf{y}$ & $\mathbf{z}$ \\
\hline $\mathrm{O}$ & -3.6486 & 3.4509 & 1.3104 & -3.4075 & 4.0914 & -0.7510 & -4.3591 & 4.5251 & -1.8824 \\
\hline $\mathrm{C}$ & -3.2434 & 2.0909 & 1.5671 & -2.0094 & 4.0137 & -0.4025 & -4.6399 & 3.1314 & -1.6418 \\
\hline $\mathrm{C}$ & -3.6719 & 1.7243 & 2.9879 & -1.2787 & 5.1809 & -1.0725 & -5.9391 & 3.0189 & -0.8413 \\
\hline C & -3.0779 & 2.6845 & 4.0223 & -1.5126 & 5.2253 & -2.5874 & -5.9111 & 3.8565 & 0.4426 \\
\hline $\mathrm{C}$ & -3.4039 & 4.1273 & 3.6408 & -3.0055 & 5.1598 & -2.8986 & -5.4821 & 5.2883 & 0.1333 \\
\hline C & -3.0368 & 4.3898 & 2.1674 & -3.6606 & 3.9996 & -2.1325 & -4.1959 & 5.2871 & -0.7095 \\
\hline $\mathrm{H}$ & -2.1524 & 2.0169 & 1.5004 & -1.5917 & 3.0686 & -0.7683 & -3.8245 & 2.6895 & -1.0578 \\
\hline $\mathrm{H}$ & -4.7677 & 1.7601 & 3.0518 & -1.6391 & 6.1220 & -0.6352 & -6.7755 & 3.3611 & -1.4655 \\
\hline $\mathrm{H}$ & -1.9844 & 2.5680 & 3.9941 & -1.0396 & 4.3319 & -3.0218 & -5.1543 & 3.4165 & 1.1085 \\
\hline $\mathrm{H}$ & -4.4838 & 4.2936 & 3.7604 & -3.4801 & 6.0984 & -2.5798 & -6.2736 & 5.7846 & -0.4455 \\
\hline $\mathrm{H}$ & -3.4127 & 5.3709 & 1.8619 & -4.7478 & 4.0467 & -2.2470 & -3.9667 & 6.3055 & -1.0375 \\
\hline C & -3.8899 & 1.1945 & 0.5199 & -1.9192 & 4.0823 & 1.1159 & -4.7459 & 2.4541 & -3.0022 \\
\hline $\mathrm{O}$ & -3.1913 & 0.4184 & 3.3122 & 0.1330 & 5.0497 & -0.8851 & -6.1330 & 1.6596 & -0.4417 \\
\hline $\mathrm{O}$ & -3.5925 & 2.3954 & 5.3108 & -0.9697 & 6.4149 & -3.1309 & -7.1913 & 3.8629 & 1.0483 \\
\hline $\mathrm{O}$ & -2.6778 & 5.0844 & 4.4020 & -3.2777 & 4.9209 & -4.2746 & -5.1863 & 6.0460 & 1.2999 \\
\hline $\mathrm{O}$ & -1.6331 & 4.3311 & 2.0481 & -3.1559 & 2.7854 & -2.6480 & -3.1486 & 4.7682 & 0.0830 \\
\hline $\mathrm{H}$ & -4.0171 & 0.1922 & 0.9361 & -0.8947 & 4.3363 & 1.4122 & -5.3043 & 1.5143 & -2.9043 \\
\hline $\mathrm{O}$ & -3.1513 & 1.1205 & -0.6912 & -2.3443 & 2.8865 & 1.7569 & -3.4776 & 2.2211 & -3.6040 \\
\hline $\mathrm{H}$ & -4.8741 & 1.5971 & 0.2592 & -2.5913 & 4.8768 & 1.4602 & -5.2991 & 3.1209 & -3.6731 \\
\hline $\mathrm{H}$ & -3.3316 & 1.4760 & 5.5263 & -0.0145 & 6.4289 & -2.9114 & -7.4119 & 2.9336 & 1.2698 \\
\hline C & -3.2544 & 5.4201 & 5.6660 & -3.2664 & 6.0802 & -5.1107 & -6.3129 & 6.6479 & 1.9413 \\
\hline C & -1.9841 & 0.3095 & -0.5930 & -1.3505 & 1.8650 & 1.7535 & -2.8417 & 1.0609 & -3.0754 \\
\hline $\mathrm{O}$ & 0.3506 & 6.2741 & -0.3573 & -5.1489 & -0.2791 & -2.9971 & 0.3500 & 5.8570 & -0.1526 \\
\hline $\mathrm{C}$ & -0.5189 & 5.2111 & 0.0737 & -4.4046 & 0.7362 & -2.3008 & -0.8212 & 5.1149 & -0.5388 \\
\hline C & -0.9301 & 5.4605 & 1.5264 & -4.0630 & 1.8722 & -3.2680 & -1.9978 & 5.5794 & 0.3228 \\
\hline
\end{tabular}




\begin{tabular}{|c|c|c|c|c|c|c|c|c|c|}
\hline $\mathrm{C}$ & 0.2946 & 5.6210 & 2.4367 & -3.3324 & 1.3561 & -4.5129 & -1.6898 & 5.4230 & 1.8182 \\
\hline $\mathrm{C}$ & 1.2900 & 6.6215 & 1.8492 & -4.0702 & 0.1640 & -5.1212 & -0.3381 & 6.0492 & 2.1645 \\
\hline $\mathrm{C}$ & 1.5600 & 6.3185 & 0.3669 & -4.4163 & -0.8759 & -4.0448 & 0.7482 & 5.5997 & 1.1759 \\
\hline $\mathrm{H}$ & 0.0215 & 4.2591 & 0.0399 & -3.4615 & 0.3145 & -1.9355 & -0.6591 & 4.0479 & -0.3491 \\
\hline $\mathrm{H}$ & -1.5472 & 6.3661 & 1.5971 & -4.9772 & 2.3909 & -3.5851 & -2.2193 & 6.6349 & 0.1160 \\
\hline $\mathrm{H}$ & 0.7968 & 4.6420 & 2.4767 & -2.3440 & 0.9994 & -4.1841 & -1.6185 & 4.3433 & 2.0229 \\
\hline $\mathrm{H}$ & 0.8592 & 7.6303 & 1.9115 & -5.0137 & 0.5160 & -5.5599 & -0.4277 & 7.1416 & 2.0893 \\
\hline $\mathrm{H}$ & 2.1512 & 7.1265 & -0.0749 & -5.0672 & -1.6456 & -4.4706 & 1.6603 & 6.1822 & 1.3378 \\
\hline $\mathrm{C}$ & -1.6876 & 5.1683 & -0.8979 & -5.2526 & 1.1860 & -1.1212 & -1.0220 & 5.3415 & -2.0287 \\
\hline $\mathrm{O}$ & -0.0963 & 6.0535 & 3.7275 & -3.2139 & 2.3760 & -5.4885 & -2.6950 & 6.0330 & 2.6071 \\
\hline $\mathrm{O}$ & 2.5488 & 6.5858 & 2.5118 & -3.2891 & -0.5012 & -6.1082 & 0.1142 & 5.6837 & 3.4634 \\
\hline $\mathrm{O}$ & 2.2473 & 5.0921 & 0.2868 & -3.2150 & -1.4394 & -3.5729 & 0.9863 & 4.2276 & 1.3826 \\
\hline $\mathrm{H}$ & -2.4837 & 4.5444 & -0.4700 & -4.8474 & 2.1274 & -0.7275 & -2.0185 & 4.9806 & -2.3169 \\
\hline $\mathrm{O}$ & -1.3127 & 4.6762 & -2.1795 & -5.3059 & 0.2137 & -0.0849 & -0.0152 & 4.7033 & -2.8072 \\
\hline $\mathrm{H}$ & -2.0654 & 6.1857 & -1.0464 & -6.2810 & 1.3474 & -1.4612 & -0.9531 & 6.4149 & -2.2373 \\
\hline $\mathrm{H}$ & -0.8836 & 5.5348 & 3.9893 & -3.0184 & 3.2150 & -5.0240 & -3.5629 & 5.8231 & 2.2064 \\
\hline $\mathrm{C}$ & 2.6520 & 7.4270 & 3.6624 & -3.4448 & -0.0016 & -7.4384 & -0.3447 & 6.5247 & 4.5235 \\
\hline $\mathrm{C}$ & -1.0748 & 3.2702 & -2.1669 & -4.0756 & 0.1154 & 0.6328 & -0.2529 & 3.3074 & -2.9629 \\
\hline $\mathrm{O}$ & 4.3493 & 3.8727 & -2.4740 & -2.6952 & -4.6479 & -1.8528 & 4.0065 & 2.3712 & 0.4334 \\
\hline $\mathrm{C}$ & 3.1205 & 4.0515 & -1.7418 & -2.9375 & -3.2299 & -1.9445 & 2.6979 & 2.9225 & 0.2246 \\
\hline $\mathrm{C}$ & 3.3880 & 4.9959 & -0.5651 & -3.1407 & -2.8562 & -3.4175 & 2.3355 & 3.7706 & 1.4490 \\
\hline $\mathrm{C}$ & 4.5217 & 4.4779 & 0.3262 & -1.9746 & -3.3160 & -4.2971 & 2.4365 & 2.9689 & 2.7528 \\
\hline $\mathrm{C}$ & 5.7535 & 4.1666 & -0.5152 & -1.7022 & -4.7969 & -4.0654 & 3.8025 & 2.2995 & 2.8457 \\
\hline $\mathrm{C}$ & 5.3847 & 3.2836 & -1.7186 & -1.5591 & -5.0901 & -2.5632 & 4.1201 & 1.5344 & 1.5575 \\
\hline $\mathrm{H}$ & 2.7762 & 3.0838 & -1.3564 & -2.0760 & -2.6812 & -1.5446 & 1.9648 & 2.1145 & 0.1080 \\
\hline $\mathrm{H}$ & 3.6657 & 5.9859 & -0.9512 & -4.0609 & -3.3324 & -3.7823 & 3.0275 & 4.6215 & 1.5106 \\
\hline $\mathrm{H}$ & 4.1793 & 3.5374 & 0.7844 & -1.0800 & -2.7544 & -3.9885 & 1.6719 & 2.1777 & 2.7186 \\
\hline $\mathrm{H}$ & 6.1600 & 5.1073 & -0.9107 & -2.5565 & -5.3793 & -4.4363 & 4.5746 & 3.0726 & 2.9580 \\
\hline $\mathrm{H}$ & 6.2438 & 3.2138 & -2.3926 & -1.5122 & -6.1720 & -2.4069 & 5.1660 & 1.2271 & 1.5838 \\
\hline $\mathrm{C}$ & 2.1029 & 4.5998 & -2.7193 & -4.1739 & -2.9397 & -1.1194 & 2.7732 & 3.7425 & -1.0463 \\
\hline $\mathrm{O}$ & 4.8588 & 5.4394 & 1.3085 & -2.2739 & -3.1041 & -5.6640 & 2.2513 & 3.8195 & 3.8695 \\
\hline $\mathrm{O}$ & 6.7509 & 3.4805 & 0.2361 & -0.5037 & -5.2244 & -4.7068 & 3.8801 & 1.3679 & 3.9229 \\
\hline $\mathrm{O}$ & 5.0131 & 2.0082 & -1.2507 & -0.3869 & -4.4665 & -2.0934 & 3.2490 & 0.4289 & 1.4553 \\
\hline $\mathrm{H}$ & 1.2169 & 4.9641 & -2.1853 & -4.5333 & -1.9228 & -1.3200 & 1.8580 & 4.3312 & -1.1754 \\
\hline $\mathrm{O}$ & 1.7468 & 3.5645 & -3.6254 & -3.8741 & -3.1002 & 0.2598 & 2.9552 & 2.8663 & -2.1525 \\
\hline $\mathrm{H}$ & 2.5554 & 5.4477 & -3.2599 & -4.9633 & -3.6472 & -1.4240 & 3.6284 & 4.4339 & -0.9645 \\
\hline $\mathrm{H}$ & 4.0326 & 5.7034 & 1.7622 & -2.5248 & -2.1636 & -5.7714 & 1.4345 & 4.3363 & 3.7172 \\
\hline $\mathrm{C}$ & 7.7165 & 4.3297 & 0.8631 & -0.6850 & -5.7351 & -6.0312 & 4.3697 & 1.9236 & 5.1467 \\
\hline $\mathrm{C}$ & 0.9485 & 4.0560 & -4.6922 & -5.0631 & -3.0630 & 1.0416 & 3.1694 & 3.5951 & -3.3543 \\
\hline $\mathrm{O}$ & 5.0215 & -1.2419 & -2.9468 & 1.7729 & -5.1716 & 0.7744 & 3.5757 & -2.7015 & -0.4275 \\
\hline $\mathrm{C}$ & 4.4167 & -0.0693 & -2.3571 & 0.8008 & -4.4292 & 0.0078 & 3.0795 & -1.3721 & -0.1623 \\
\hline $\mathrm{C}$ & 5.5465 & 0.8738 & -1.9382 & 0.4782 & -5.2323 & -1.2519 & 3.8242 & -0.8138 & 1.0509 \\
\hline $\mathrm{C}$ & 6.5258 & 0.1967 & -0.9703 & 1.7383 & -5.5417 & -2.0715 & 3.8041 & -1.7344 & 2.2727 \\
\hline $\mathrm{C}$ & 7.0095 & -1.1351 & -1.5359 & 2.8207 & -6.1629 & -1.1920 & 4.1982 & -3.1545 & 1.8788 \\
\hline $\mathrm{C}$ & 5.8273 & -1.9699 & -2.0518 & 2.9974 & -5.3547 & 0.1030 & 3.4039 & -3.5994 & 0.6424 \\
\hline $\mathrm{H}$ & 3.8432 & -0.3595 & -1.4692 & 1.2254 & -3.4623 & -0.2863 & 2.0047 & -1.4146 & 0.0467 \\
\hline $\mathrm{H}$ & 6.0944 & 1.1889 & -2.8362 & 0.0002 & -6.1772 & -0.9612 & 4.8688 & -0.6778 & 0.7585 \\
\hline $\mathrm{H}$ & 5.9749 & -0.0130 & -0.0411 & 2.1245 & -4.5834 & -2.4498 & 2.7770 & -1.7641 & 2.6629 \\
\hline $\mathrm{H}$ & 7.6886 & -0.9380 & -2.3774 & 2.5207 & -7.1861 & -0.9254 & 5.2657 & -3.1789 & 1.6242 \\
\hline $\mathrm{H}$ & 6.2061 & -2.8316 & -2.6092 & 3.6472 & -5.9001 & 0.7938 & 3.7759 & -4.5670 & 0.2918 \\
\hline C & 3.4797 & 0.5345 & -3.3973 & -0.4104 & -4.1997 & 0.9044 & 3.3664 & -0.5342 & -1.4018 \\
\hline $\mathrm{O}$ & 7.6419 & 1.0351 & -0.7328 & 1.4294 & -6.4289 & -3.1309 & 4.7102 & -1.2398 & 3.2495 \\
\hline $\mathrm{O}$ & 7.6597 & -1.9487 & -0.5648 & 4.1011 & -6.1754 & -1.8150 & 3.9125 & -4.1114 & 2.8935 \\
\hline $\mathrm{O}$ & 5.0580 & -2.3883 & -0.9456 & 3.5578 & -4.1048 & -0.2309 & 2.0418 & -3.6868 & 1.0023 \\
\hline $\mathrm{H}$ & 3.3294 & 1.6015 & -3.1918 & -1.3157 & -4.0873 & 0.2943 & 3.3314 & 0.5308 & -1.1434 \\
\hline $\mathrm{O}$ & 2.2300 & -0.1488 & -3.4578 & -0.2340 & -3.0672 & 1.7533 & 2.4907 & -0.8167 & -2.4855 \\
\hline
\end{tabular}




\begin{tabular}{|c|c|c|c|c|c|c|c|c|c|}
\hline $\mathrm{H}$ & 3.9480 & 0.4336 & -4.3829 & -0.5342 & -5.0722 & 1.5545 & 4.3792 & -0.7654 & -1.7447 \\
\hline $\mathrm{H}$ & 7.3060 & 1.8661 & -0.3349 & 0.7851 & -5.9730 & -3.7133 & 4.3930 & -0.3504 & 3.5157 \\
\hline C & 9.0425 & -1.6555 & -0.3537 & 4.3383 & -7.2780 & -2.6928 & 4.9191 & -4.2377 & 3.8999 \\
\hline C & 1.3122 & 0.3049 & -2.4648 & -0.5911 & -1.8481 & 1.1079 & 1.2434 & -0.1422 & -2.3624 \\
\hline $\mathrm{O}$ & 3.4448 & -5.6847 & -0.7808 & 5.9291 & -2.3420 & 1.9469 & -0.2884 & -6.1506 & -0.4077 \\
\hline C & 3.7034 & -4.3701 & -1.3089 & 4.6639 & -2.9076 & 1.5612 & 0.5507 & -4.9816 & -0.4048 \\
\hline C & 4.9671 & -3.7853 & -0.6668 & 4.8355 & -3.7613 & 0.3038 & 1.3774 & -4.9441 & 0.8824 \\
\hline C & 4.9805 & -3.8757 & 0.8627 & 5.5365 & -3.0050 & -0.8292 & 0.5048 & -5.0552 & 2.1364 \\
\hline $\mathrm{C}$ & 4.5724 & -5.2707 & 1.3282 & 6.7953 & -2.3060 & -0.3213 & -0.4682 & -6.2254 & 2.0137 \\
\hline C & 3.2833 & -5.7064 & 0.6202 & 6.4955 & -1.5114 & 0.9586 & -1.2031 & -6.1883 & 0.6650 \\
\hline $\mathrm{H}$ & 2.8575 & -3.7083 & -1.0886 & 3.9546 & -2.1051 & 1.3251 & -0.0739 & -4.0803 & -0.4336 \\
\hline $\mathrm{H}$ & 5.8552 & -4.3095 & -1.0452 & 5.4149 & -4.6660 & 0.5311 & 2.1046 & -5.7671 & 0.8885 \\
\hline $\mathrm{H}$ & 4.2259 & -3.1702 & 1.2406 & 4.8441 & -2.2230 & -1.1763 & -0.0922 & -4.1327 & 2.2024 \\
\hline $\mathrm{H}$ & 5.3623 & -5.9874 & 1.0657 & 7.5512 & -3.0635 & -0.0735 & 0.0985 & -7.1653 & 2.0576 \\
\hline $\mathrm{H}$ & 3.0486 & -6.7421 & 0.8836 & 7.4319 & -1.1344 & 1.3809 & -1.7773 & -7.1110 & 0.5375 \\
\hline C & 3.8588 & -4.5382 & -2.8149 & 4.1582 & -3.6904 & 2.7650 & 1.4091 & -5.0534 & -1.6606 \\
\hline $\mathrm{O}$ & 6.2717 & -3.5644 & 1.3609 & 5.8760 & -3.8955 & -1.8795 & 1.3091 & -5.2328 & 3.2898 \\
\hline $\mathrm{O}$ & 4.3104 & -5.3168 & 2.7288 & 7.3179 & -1.3819 & -1.2717 & -1.4636 & -6.2046 & 3.0323 \\
\hline $\mathrm{O}$ & 2.2547 & -4.8384 & 1.0314 & 5.6330 & -0.4488 & 0.6265 & -2.0538 & -5.0652 & 0.6574 \\
\hline $\mathrm{H}$ & 4.2620 & -3.6131 & -3.2446 & 3.3263 & -4.3386 & 2.4653 & 2.2397 & -4.3412 & -1.5790 \\
\hline $\mathrm{O}$ & 2.6353 & -4.8790 & -3.4466 & 3.7543 & -2.8080 & 3.8048 & 0.6461 & -4.7927 & -2.8324 \\
\hline $\mathrm{H}$ & 4.5697 & -5.3533 & -2.9971 & 4.9751 & -4.3105 & 3.1510 & 1.8133 & -6.0679 & -1.7499 \\
\hline $\mathrm{H}$ & 6.6313 & -2.8360 & 0.8165 & 5.1355 & -4.5255 & -1.9832 & 2.0928 & -4.6541 & 3.2016 \\
\hline C & 5.4520 & -5.6036 & 3.5408 & 8.2568 & -1.9406 & -2.1943 & -1.1149 & -6.9079 & 4.2271 \\
\hline $\mathrm{C}$ & 1.9339 & -3.7270 & -3.9060 & 2.3874 & -2.4311 & 3.6831 & 0.5481 & -3.3977 & -3.0959 \\
\hline $\mathrm{O}$ & -1.3870 & -5.1074 & 0.9511 & 4.9420 & 2.6685 & 2.4200 & -4.9993 & -4.4499 & -1.4377 \\
\hline C & -0.0871 & -4.6152 & 0.5579 & 4.6427 & 1.3515 & 1.9211 & -3.6284 & -4.2880 & -1.0277 \\
\hline C & 0.9787 & -5.4104 & 1.3074 & 5.8935 & 0.8231 & 1.2211 & -3.3765 & -5.2331 & 0.1461 \\
\hline C & 0.7792 & -5.3410 & 2.8244 & 6.3082 & 1.7573 & 0.0758 & -4.3313 & -4.9265 & 1.3077 \\
\hline $\mathrm{C}$ & -0.6441 & -5.7604 & 3.1807 & 6.4323 & 3.1978 & 0.5719 & -5.7796 & -4.9124 & 0.8182 \\
\hline C & -1.6606 & -4.9788 & 2.3272 & 5.1940 & 3.6020 & 1.3930 & -5.9249 & -4.0531 & -0.4505 \\
\hline $\mathrm{H}$ & 0.0071 & -3.5584 & 0.8361 & 3.8404 & 1.4147 & 1.1787 & -3.4689 & -3.2603 & -0.6839 \\
\hline $\mathrm{H}$ & 0.9443 & -6.4598 & 0.9853 & 6.7135 & 0.7523 & 1.9474 & -3.5362 & -6.2679 & -0.1841 \\
\hline $\mathrm{H}$ & 0.9063 & -4.2912 & 3.1270 & 5.5035 & 1.7298 & -0.6744 & -4.0891 & -3.9175 & 1.6751 \\
\hline $\mathrm{H}$ & -0.7679 & -6.8314 & 2.9670 & 7.3102 & 3.2708 & 1.2292 & -6.0729 & -5.9405 & 0.5630 \\
\hline $\mathrm{H}$ & -2.6619 & -5.3939 & 2.4760 & 5.3760 & 4.5596 & 1.8900 & -6.9171 & -4.2039 & -0.8863 \\
\hline C & 0.0287 & -4.7393 & -0.9660 & 4.1881 & 0.5047 & 3.0974 & -2.7492 & -4.5734 & -2.2343 \\
\hline $\mathrm{O}$ & 1.7132 & -6.1813 & 3.4800 & 7.5422 & 1.3363 & -0.4775 & -4.1900 & -5.8966 & 2.3293 \\
\hline $\mathrm{O}$ & -0.9842 & -5.4940 & 4.5353 & 6.5366 & 4.1422 & -0.4861 & -6.6841 & -4.3723 & 1.7730 \\
\hline $\mathrm{O}$ & -1.6215 & -3.6202 & 2.7140 & 4.0938 & 3.6931 & 0.5170 & -5.7340 & -2.7003 & -0.0988 \\
\hline $\mathrm{H}$ & 1.0367 & -5.0533 & -1.2464 & 4.1624 & -0.5466 & 2.7927 & -1.7000 & -4.5753 & -1.9171 \\
\hline $\mathrm{O}$ & -0.1958 & -3.5120 & -1.6427 & 2.9237 & 0.9003 & 3.6273 & -2.9444 & -3.6625 & -3.3121 \\
\hline $\mathrm{H}$ & -0.6883 & -5.5062 & -1.3017 & 4.9070 & 0.6086 & 3.9165 & -2.9915 & -5.5656 & -2.6295 \\
\hline $\mathrm{H}$ & 2.6066 & -5.8351 & 3.2757 & 7.4171 & 0.4230 & -0.8088 & -3.2473 & -5.9092 & 2.5954 \\
\hline C & -0.5934 & -6.5005 & 5.4722 & 7.8583 & 4.3460 & -0.9899 & -7.1623 & -5.2980 & 2.7511 \\
\hline C & -1.5052 & -2.9995 & -1.4414 & 1.8573 & 0.8246 & 2.6873 & -2.7179 & -2.3034 & -2.9636 \\
\hline $\mathrm{O}$ & -4.5486 & -1.3965 & 2.7662 & 1.1237 & 5.7888 & 1.0891 & -7.2187 & 0.1691 & -1.8643 \\
\hline $\mathrm{C}$ & -3.4677 & -2.1393 & 2.1716 & 1.9443 & 4.6140 & 1.2320 & -6.2669 & -0.8884 & -1.6449 \\
\hline C & -2.8098 & -3.0203 & 3.2376 & 3.2882 & 4.8710 & 0.5424 & -6.7323 & -1.7455 & -0.4618 \\
\hline C & -2.3545 & -2.1914 & 4.4461 & 3.0976 & 5.2602 & -0.9270 & -6.9639 & -0.8985 & 0.7935 \\
\hline C & -3.4879 & -1.2955 & 4.9460 & 2.0986 & 6.4073 & -1.0464 & -7.8678 & 0.2881 & 0.4710 \\
\hline C & -4.1283 & -0.5165 & 3.7895 & 0.8171 & 6.1052 & -0.2523 & -7.3575 & 1.0450 & -0.7652 \\
\hline $\mathrm{H}$ & -2.7090 & -1.4432 & 1.7957 & 1.4520 & 3.7605 & 0.7492 & -5.2862 & -0.4581 & -1.4070 \\
\hline $\mathrm{H}$ & -3.5116 & -3.7913 & 3.5818 & 3.8146 & 5.6855 & 1.0578 & -7.6716 & -2.2519 & -0.7211 \\
\hline $\mathrm{H}$ & -1.5392 & -1.5361 & 4.1022 & 2.6722 & 4.3880 & -1.4471 & -5.9870 & -0.5007 & 1.1091 \\
\hline $\mathrm{H}$ & -4.2672 & -1.9285 & 5.3916 & 2.5471 & 7.3128 & -0.6160 & -8.8741 & -0.0851 & 0.2379 \\
\hline
\end{tabular}




\begin{tabular}{|c|c|c|c|c|c|c|c|c|c|}
\hline $\mathrm{H}$ & -5.0332 & -0.0138 & 4.1430 & 0.1933 & 7.0031 & -0.2121 & -8.0985 & 1.7906 & -1.0686 \\
\hline $\mathrm{C}$ & -4.0361 & -2.9188 & 0.9948 & 2.0804 & 4.3344 & 2.7140 & -6.1670 & -1.6820 & -2.9305 \\
\hline $\mathrm{O}$ & -1.9313 & -3.0310 & 5.5043 & 4.3302 & 5.6542 & -1.5011 & -7.5615 & -1.6735 & 1.8160 \\
\hline $\mathrm{O}$ & -3.0340 & -0.3335 & 5.8949 & 1.7195 & 6.6442 & -2.3986 & -7.9275 & 1.2177 & 1.5493 \\
\hline $\mathrm{H}$ & -3.2953 & -3.6544 & 0.6725 & 2.8331 & 3.5517 & 2.8714 & -5.6071 & -2.6076 & -2.7429 \\
\hline $\mathrm{O}$ & -4.2903 & -2.0694 & -0.1194 & 0.8174 & 3.9141 & 3.2034 & -5.4995 & -0.8917 & -3.9020 \\
\hline $\mathrm{H}$ & -4.9474 & -3.4480 & 1.3081 & 2.4140 & 5.2494 & 3.2312 & -7.1795 & -1.9494 & -3.2748 \\
\hline $\mathrm{H}$ & -1.4178 & -3.7687 & 5.1172 & 5.0032 & 4.9919 & -1.2452 & -7.0863 & -2.5277 & 1.8605 \\
\hline $\mathrm{C}$ & -3.0868 & -0.7676 & 7.2570 & 2.5232 & 7.6072 & -3.0860 & -8.9921 & 0.9889 & 2.4775 \\
\hline $\mathrm{C}$ & -5.6270 & -1.5748 & -0.1849 & 0.8755 & 3.5258 & 4.5687 & -5.2627 & -1.6249 & -5.0959 \\
\hline $\mathrm{H}$ & -1.6172 & 0.1577 & -1.6113 & -1.7175 & 1.0634 & 2.4020 & -1.9232 & 0.9062 & -3.6486 \\
\hline $\mathrm{H}$ & -1.2015 & 0.7896 & 0.0117 & -1.1873 & 1.4564 & 0.7445 & -2.5728 & 1.1858 & -2.0157 \\
\hline $\mathrm{H}$ & -2.2418 & -0.6622 & -0.1635 & -0.4051 & 2.2538 & 2.1450 & -3.5003 & 0.1926 & -3.1805 \\
\hline $\mathrm{H}$ & -1.0438 & 2.9392 & -3.2061 & -4.3112 & -0.3415 & 1.5950 & 0.5169 & 2.9233 & -3.6350 \\
\hline $\mathrm{H}$ & -0.1110 & 3.0244 & -1.7030 & -3.3488 & -0.5127 & 0.1028 & -0.1745 & 2.7681 & -2.0081 \\
\hline $\mathrm{H}$ & -1.8770 & 2.7478 & -1.6350 & -3.6491 & 1.1106 & 0.8034 & -1.2501 & 3.1334 & -3.3860 \\
\hline $\mathrm{H}$ & 0.6552 & 3.1954 & -5.3003 & -4.7744 & -3.1780 & 2.0871 & 3.2472 & 2.8657 & -4.1651 \\
\hline $\mathrm{H}$ & 0.0494 & 4.5614 & -4.3141 & -5.5933 & -2.1101 & 0.9090 & 2.3353 & 4.2819 & -3.5509 \\
\hline $\mathrm{H}$ & 1.5170 & 4.7646 & -5.3149 & -5.7398 & -3.8842 & 0.7566 & 4.1050 & 4.1731 & -3.2981 \\
\hline $\mathrm{H}$ & 0.3778 & -0.2417 & -2.6171 & -0.4663 & -1.0508 & 1.8444 & 0.6494 & -0.4002 & -3.2442 \\
\hline $\mathrm{H}$ & 1.6811 & 0.1055 & -1.4470 & 0.0556 & -1.6437 & 0.2417 & 0.6908 & -0.4630 & -1.4656 \\
\hline $\mathrm{H}$ & 1.1345 & 1.3834 & -2.5736 & -1.6379 & -1.8827 & 0.7768 & 1.3953 & 0.9447 & -2.3174 \\
\hline $\mathrm{H}$ & 2.5072 & -3.2078 & -4.6895 & 2.2498 & -1.5206 & 4.2692 & -0.1709 & -3.2742 & -3.9093 \\
\hline $\mathrm{H}$ & 0.9855 & -4.0784 & -4.3171 & 2.1046 & -2.2290 & 2.6421 & 0.1848 & -2.8463 & -2.2176 \\
\hline $\mathrm{H}$ & 1.7230 & -3.0197 & -3.0968 & 1.7239 & -3.2274 & 4.0511 & 1.5216 & -2.9682 & -3.3673 \\
\hline $\mathrm{H}$ & -1.5974 & -2.1025 & -2.0549 & 0.9309 & 0.8651 & 3.2601 & -2.6438 & -1.7448 & -3.8982 \\
\hline $\mathrm{H}$ & -2.2673 & -3.7377 & -1.7356 & 1.8755 & 1.6656 & 1.9837 & -3.5470 & -1.8914 & -2.3772 \\
\hline $\mathrm{H}$ & -1.6771 & -2.7398 & -0.3909 & 1.8903 & -0.1155 & 2.1199 & -1.7885 & -2.1792 & -2.3938 \\
\hline $\mathrm{H}$ & -5.6704 & -0.9060 & -1.0472 & -0.1173 & 3.1613 & 4.8416 & -4.7092 & -0.9689 & -5.7733 \\
\hline $\mathrm{H}$ & -6.3351 & -2.4010 & -0.3369 & 1.6086 & 2.7207 & 4.7168 & -4.6686 & -2.5273 & -4.8908 \\
\hline $\mathrm{H}$ & -5.8905 & -1.0258 & 0.7252 & 1.1448 & 4.3788 & 5.2114 & -6.2095 & -1.9234 & -5.5720 \\
\hline $\mathrm{H}$ & -2.6443 & 6.2278 & 6.0782 & -3.5902 & 5.7484 & -6.1005 & -5.9123 & 7.2764 & 2.7408 \\
\hline $\mathrm{H}$ & -4.2864 & 5.7740 & 5.5331 & -3.9710 & 6.8320 & -4.7289 & -6.8678 & 7.2737 & 1.2284 \\
\hline $\mathrm{H}$ & -3.2523 & 4.5585 & 6.3395 & -2.2654 & 6.5173 & -5.1624 & -6.9831 & 5.8879 & 2.3520 \\
\hline $\mathrm{H}$ & 3.6892 & 7.3632 & 4.0007 & -2.8513 & -0.6525 & -8.0852 & 0.1577 & 6.1810 & 5.4312 \\
\hline $\mathrm{H}$ & 2.4174 & 8.4682 & 3.3998 & -4.4991 & -0.0451 & -7.7455 & -0.0716 & 7.5717 & 4.3301 \\
\hline $\mathrm{H}$ & 1.9736 & 7.0918 & 4.4523 & -3.0888 & 1.0303 & -7.5089 & -1.4299 & 6.4502 & 4.6390 \\
\hline $\mathrm{H}$ & 8.4446 & 3.6698 & 1.3411 & 0.2966 & -6.0786 & -6.3669 & 4.4304 & 1.0986 & 5.8608 \\
\hline $\mathrm{H}$ & 8.2248 & 4.9547 & 0.1157 & -1.3867 & -6.5807 & -6.0265 & 5.3697 & 2.3563 & 5.0017 \\
\hline $\mathrm{H}$ & 7.2365 & 4.9698 & 1.6087 & -1.0610 & -4.9512 & -6.6945 & 3.6885 & 2.6958 & 5.5156 \\
\hline $\mathrm{H}$ & 9.4171 & -2.4171 & 0.3350 & 5.3813 & -7.2003 & -3.0102 & 4.6041 & -5.0558 & 4.5525 \\
\hline $\mathrm{H}$ & 9.5950 & -1.7172 & -1.3018 & 4.1885 & -8.2286 & -2.1617 & 5.8865 & -4.4895 & 3.4425 \\
\hline $\mathrm{H}$ & 9.1718 & -0.6571 & 0.0734 & 3.6718 & -7.2417 & -3.5592 & 5.0219 & -3.3111 & 4.4719 \\
\hline $\mathrm{H}$ & 5.0893 & -5.6839 & 4.5685 & 8.6241 & -1.1112 & -2.8035 & -1.9937 & -6.8715 & 4.8756 \\
\hline $\mathrm{H}$ & 5.9114 & -6.5557 & 3.2399 & 9.0986 & -2.4009 & -1.6580 & -0.8707 & -7.9555 & 4.0015 \\
\hline $\mathrm{H}$ & 6.1928 & -4.8026 & 3.4614 & 7.7756 & -2.6920 & -2.8272 & -0.2606 & -6.4340 & 4.7192 \\
\hline $\mathrm{H}$ & -1.0084 & -6.1998 & 6.4375 & 7.7909 & 5.1627 & -1.7131 & -7.9181 & -4.7669 & 3.3352 \\
\hline $\mathrm{H}$ & -1.0122 & -7.4742 & 5.1820 & 8.5383 & 4.6349 & -0.1760 & -7.6238 & -6.1671 & 2.2618 \\
\hline $\mathrm{H}$ & 0.4956 & -6.5787 & 5.5322 & 8.2375 & 3.4404 & -1.4719 & -6.3494 & -5.6382 & 3.3988 \\
\hline $\mathrm{H}$ & -2.7888 & 0.0890 & 7.8665 & 2.0778 & 7.7316 & -4.0761 & -8.9511 & 1.8057 & 3.2022 \\
\hline $\mathrm{H}$ & -4.1081 & -1.0706 & 7.5271 & 2.5107 & 8.5697 & -2.5560 & -9.9631 & 1.0045 & 1.9634 \\
\hline $\mathrm{H}$ & -2.4039 & -1.6061 & 7.4205 & 3.5544 & 7.2537 & -3.1752 & -8.8613 & 0.0271 & 2.9812 \\
\hline $\mathrm{C}$ & -5.5224 & 2.0391 & -2.6530 & -6.0241 & -2.6239 & 5.4075 & 5.5908 & 0.8970 & -3.4116 \\
\hline $\mathrm{C}$ & -5.1809 & -3.1938 & -2.9177 & -2.1162 & 0.9173 & 5.8804 & 7.4219 & -1.5907 & 0.8207 \\
\hline $\mathrm{C}$ & -7.9500 & 1.2331 & -3.7678 & -3.7019 & -4.0465 & 4.8246 & 7.3010 & -1.0459 & -4.4475 \\
\hline $\mathrm{C}$ & -6.7116 & 2.6702 & -2.2758 & -5.8864 & -3.9469 & 5.8392 & 5.2334 & 0.1919 & -4.5641 \\
\hline
\end{tabular}




\begin{tabular}{|c|c|c|c|c|c|c|c|c|c|}
\hline C & -7.4179 & -2.8626 & -4.5048 & -0.6854 & -1.0763 & 4.6078 & 9.1192 & -2.6742 & -1.0746 \\
\hline $\mathrm{C}$ & -6.1299 & -4.2121 & -2.9832 & -0.7416 & 0.8201 & 6.0904 & 7.9267 & -2.8774 & 1.0065 \\
\hline $\mathrm{C}$ & -7.9295 & 2.2689 & -2.8277 & -4.7238 & -4.6630 & 5.5543 & 6.0865 & -0.7796 & -5.0891 \\
\hline $\mathrm{C}$ & -7.2562 & -4.0506 & -3.7925 & -0.0153 & -0.1774 & 5.4372 & 8.7854 & -3.4254 & 0.0521 \\
\hline C & -0.6266 & -1.5082 & -4.8094 & -5.7056 & 3.3112 & 2.9235 & 7.7741 & 3.4585 & 2.0325 \\
\hline $\mathrm{C}$ & -3.1444 & 1.1483 & -3.9653 & -6.4847 & -0.1923 & 4.0092 & 6.6179 & 2.6652 & -1.4510 \\
\hline $\mathrm{C}$ & -2.9613 & -1.6459 & -4.2959 & -4.3353 & 1.6302 & 3.9335 & 7.8515 & 1.4173 & 0.7665 \\
\hline $\mathrm{C}$ & -1.9911 & 0.4859 & -4.7077 & -6.5245 & 1.0619 & 3.1461 & 7.2819 & 3.4508 & -0.3278 \\
\hline C & -6.7512 & -0.5274 & -5.1625 & -2.7493 & -2.0607 & 3.5711 & 8.9500 & -0.6416 & -2.5534 \\
\hline $\mathrm{C}$ & -5.5420 & 0.9984 & -3.5944 & -4.9999 & -2.0023 & 4.6782 & 6.8095 & 0.6339 & -2.7694 \\
\hline $\mathrm{C}$ & -5.3215 & -2.0029 & -3.6467 & -2.7971 & 0.0377 & 5.0246 & 7.7568 & -0.8233 & -0.3080 \\
\hline $\mathrm{C}$ & -6.7699 & 0.6034 & -4.1643 & -3.8297 & -2.7316 & 4.3774 & 7.6732 & -0.3464 & -3.2995 \\
\hline C & -6.4672 & -1.8339 & -4.4465 & -2.0688 & -0.9882 & 4.3950 & 8.6216 & -1.3783 & -1.2691 \\
\hline $\mathrm{C}$ & -4.1502 & -1.0266 & -3.5391 & -4.2722 & 0.3565 & 4.7972 & 7.1768 & 0.5883 & -0.3419 \\
\hline $\mathrm{N}$ & -1.7489 & -0.8440 & -4.1541 & -5.7039 & 2.1103 & 3.7532 & 7.1966 & 2.7078 & 0.9267 \\
\hline $\mathrm{N}$ & -4.3694 & 0.3425 & -4.0626 & -5.1090 & -0.6840 & 4.1547 & 7.2492 & 1.3427 & -1.6142 \\
\hline $\mathrm{H}$ & -4.5852 & 2.3382 & -2.2008 & -6.9320 & -2.0795 & 5.6404 & 4.9010 & 1.6183 & -2.9924 \\
\hline $\mathrm{H}$ & -4.3260 & -3.2976 & -2.2587 & -2.6811 & 1.6983 & 6.3833 & 6.7598 & -1.1765 & 1.5772 \\
\hline $\mathrm{H}$ & -8.8900 & 0.9166 & -4.2132 & -2.7960 & -4.5951 & 4.5791 & 7.9754 & -1.8012 & -4.8436 \\
\hline $\mathrm{H}$ & -6.6804 & 3.4723 & -1.5433 & -6.6913 & -4.4112 & 6.4021 & 4.2771 & 0.3955 & -5.0372 \\
\hline $\mathrm{H}$ & -8.3036 & -2.7148 & -5.1177 & -0.1445 & -1.8729 & 4.1064 & 9.7765 & -3.0951 & -1.8311 \\
\hline $\mathrm{H}$ & -5.9924 & -5.1190 & -2.4014 & -0.2435 & 1.5187 & 6.7555 & 7.6505 & -3.4427 & 1.8919 \\
\hline $\mathrm{H}$ & -8.8542 & 2.7575 & -2.5343 & -4.6145 & -5.6911 & 5.8867 & 5.8114 & -1.3284 & -5.9851 \\
\hline $\mathrm{H}$ & -8.0085 & -4.8317 & -3.8562 & 1.0596 & -0.2481 & 5.5708 & 9.1856 & -4.4269 & 0.1788 \\
\hline $\mathrm{H}$ & 0.2761 & -0.8969 & -4.7194 & -6.7343 & 3.6693 & 2.8010 & 7.2418 & 4.4095 & 2.1445 \\
\hline $\mathrm{H}$ & -0.4466 & -2.4656 & -4.3122 & -5.1152 & 4.0955 & 3.4110 & 7.6672 & 2.8852 & 2.9602 \\
\hline $\mathrm{H}$ & -0.8229 & -1.6949 & -5.8823 & -5.2720 & 3.1335 & 1.9225 & 8.8488 & 3.6782 & 1.8813 \\
\hline $\mathrm{H}$ & -3.3495 & 2.1305 & -4.4027 & -7.0824 & -0.9804 & 3.5430 & 6.7299 & 3.2071 & -2.3952 \\
\hline $\mathrm{H}$ & -2.8526 & 1.2973 & -2.9234 & -6.9293 & 0.0426 & 4.9923 & 5.5499 & 2.5753 & -1.2210 \\
\hline $\mathrm{H}$ & -2.7689 & -2.6436 & -3.8946 & -3.7544 & 2.4179 & 4.4222 & 7.7814 & 0.8710 & 1.7119 \\
\hline $\mathrm{H}$ & -3.2459 & -1.7558 & -5.3606 & -3.8486 & 1.4183 & 2.9725 & 8.9256 & 1.5240 & 0.5143 \\
\hline $\mathrm{H}$ & -2.2237 & 0.4293 & -5.7888 & -6.1933 & 0.8198 & 2.1220 & 8.3375 & 3.6616 & -0.5909 \\
\hline $\mathrm{H}$ & -1.0851 & 1.0906 & -4.5850 & -7.5600 & 1.4189 & 3.0877 & 6.7626 & 4.4083 & -0.2087 \\
\hline $\mathrm{H}$ & -7.7123 & -0.5946 & -5.6813 & -2.0084 & -2.7917 & 3.2433 & 9.6202 & -1.2515 & -3.1664 \\
\hline $\mathrm{H}$ & -5.9654 & -0.3302 & -5.9020 & -3.1973 & -1.6224 & 2.6748 & 9.4512 & 0.3056 & -2.3190 \\
\hline $\mathrm{H}$ & -3.8666 & -0.9636 & -2.4751 & -4.7147 & 0.6008 & 5.7804 & 6.1145 & 0.5064 & -0.0717 \\
\hline
\end{tabular}


Table S13. Cartesian coordinates of atoms in 11 different structures of MIA:DM- $\beta-C D$ in water (PCM) obtained from the M062X-GD3/6-31G(d,p) calculations performed using in the initial model of the complex the optimized structure BOYFOK03 of DM- $\beta-C D$. The true minima are confirmed by zero imaginary frequencies.

\begin{tabular}{|c|c|c|c|c|c|c|c|c|c|}
\hline \multirow[b]{2}{*}{ atom } & \multicolumn{3}{|c|}{ CR1 } & \multicolumn{3}{|c|}{ NR1 } & \multicolumn{3}{|c|}{ M1 } \\
\hline & $\mathbf{x}$ & $\mathbf{y}$ & $\mathbf{Z}$ & $\mathbf{x}$ & $\mathbf{y}$ & $\mathbf{z}$ & $\mathbf{x}$ & $\mathbf{y}$ & $\mathbf{Z}$ \\
\hline $\mathrm{C}$ & -3.1096 & 5.1866 & -0.2202 & 5.8196 & 1.3628 & -0.4678 & 2.5087 & 5.1552 & -0.0242 \\
\hline $\mathrm{C}$ & -2.2596 & 5.2913 & -1.4974 & 5.6463 & 2.3537 & 0.6876 & 1.8437 & 5.1178 & 1.3586 \\
\hline $\mathrm{C}$ & -0.8394 & 4.7858 & -1.2809 & 4.3160 & 3.0782 & 0.5764 & 0.4884 & 4.4313 & 1.2862 \\
\hline $\mathrm{C}$ & -0.2597 & 5.4187 & -0.0244 & 4.1042 & 3.6241 & -0.8272 & -0.3332 & 5.0773 & 0.1810 \\
\hline C & -1.1854 & 5.1762 & 1.1650 & 4.3333 & 2.5458 & -1.8800 & 0.4175 & 5.0366 & -1.1461 \\
\hline $\mathrm{C}$ & -0.6364 & 5.7524 & 2.4611 & 4.1781 & 3.0730 & -3.2997 & -0.3797 & 5.6790 & -2.2718 \\
\hline $\mathrm{C}$ & -2.6536 & 4.9632 & -3.8460 & 6.2797 & 2.3241 & 2.9956 & 2.6211 & 4.8240 & 3.6070 \\
\hline C & -1.1044 & 8.0688 & 2.1892 & 6.2044 & 4.2830 & -3.5600 & -0.0948 & 7.9939 & -1.8066 \\
\hline $\mathrm{O}$ & -2.9369 & 4.5655 & -2.5069 & 5.7295 & 1.6140 & 1.8921 & 2.7309 & 4.4479 & 2.2362 \\
\hline $\mathrm{O}$ & -0.0665 & 5.1064 & -2.4158 & 4.2794 & 4.1190 & 1.5249 & -0.1515 & 4.5576 & 2.5312 \\
\hline $\mathrm{O}$ & 1.0049 & 4.8132 & 0.1758 & 2.7588 & 4.0645 & -0.8507 & -1.5551 & 4.3656 & 0.0976 \\
\hline $\mathrm{O}$ & -2.4540 & 5.7721 & 0.8764 & 5.6458 & 2.0027 & -1.7099 & 1.6595 & 5.7282 & -0.9874 \\
\hline $\mathrm{O}$ & -0.1214 & 7.0604 & 2.3445 & 4.7885 & 4.3231 & -3.5295 & -0.9893 & 6.9016 & -1.9216 \\
\hline $\mathrm{H}$ & -4.0388 & 5.7495 & -0.3548 & 6.8393 & 0.9644 & -0.4710 & 3.3987 & 5.7910 & 0.0111 \\
\hline $\mathrm{H}$ & -2.2041 & 6.3549 & -1.7744 & 6.4629 & 3.0878 & 0.6332 & 1.6932 & 6.1548 & 1.6943 \\
\hline $\mathrm{H}$ & -0.8703 & 3.6966 & -1.1195 & 3.5155 & 2.3437 & 0.7639 & 0.6519 & 3.3706 & 1.0225 \\
\hline $\mathrm{H}$ & -0.1455 & 6.5002 & -0.1822 & 4.7891 & 4.4657 & -0.9986 & -0.5242 & 6.1250 & 0.4508 \\
\hline $\mathrm{H}$ & -1.3149 & 4.0914 & 1.3070 & 3.5879 & 1.7491 & -1.7334 & 0.6152 & 3.9876 & -1.4209 \\
\hline $\mathrm{H}$ & 0.2040 & 5.1263 & 2.7780 & 3.1093 & 3.2201 & -3.4863 & -1.2031 & 5.0061 & -2.5314 \\
\hline $\mathrm{H}$ & -3.3816 & 4.4524 & -4.4777 & 6.3233 & 1.6193 & 3.8269 & 3.4207 & 4.3014 & 4.1335 \\
\hline $\mathrm{H}$ & -2.7722 & 6.0468 & -3.9579 & 7.2924 & 2.6731 & 2.7631 & 2.7578 & 5.9058 & 3.7206 \\
\hline $\mathrm{H}$ & -1.6401 & 4.6778 & -4.1310 & 5.6499 & 3.1760 & 3.2608 & 1.6504 & 4.5326 & 4.0103 \\
\hline $\mathrm{H}$ & -0.6066 & 9.0260 & 2.3523 & 6.5419 & 5.2434 & -3.9524 & -0.7020 & 8.9001 & -1.7734 \\
\hline $\mathrm{H}$ & -1.5501 & 8.0543 & 1.1894 & 6.6297 & 4.1259 & -2.5631 & 0.5152 & 7.9303 & -0.8997 \\
\hline $\mathrm{H}$ & -1.9070 & 7.9480 & 2.9280 & 6.5574 & 3.4780 & -4.2174 & 0.5775 & 8.0411 & -2.6731 \\
\hline $\mathrm{H}$ & 0.8664 & 5.1700 & -2.1384 & 3.5877 & 4.7470 & 1.2438 & -1.1072 & 4.4111 & 2.3992 \\
\hline C & 2.0645 & 5.6321 & 0.6175 & 2.4427 & 5.2351 & -1.5629 & -2.7423 & 5.1187 & -0.0080 \\
\hline C & 3.1329 & 5.6535 & -0.4790 & 1.8762 & 6.2390 & -0.5540 & -3.6382 & 4.7528 & 1.1768 \\
\hline $\mathrm{C}$ & 3.7484 & 4.2726 & -0.6320 & 0.5637 & 5.7126 & 0.0045 & -4.0823 & 3.3005 & 1.0837 \\
\hline $\mathrm{C}$ & 4.2036 & 3.7304 & 0.7212 & -0.3766 & 5.2906 & -1.1226 & -4.6364 & 3.0142 & -0.3127 \\
\hline C & 3.0600 & 3.7946 & 1.7325 & 0.3191 & 4.3310 & -2.0822 & -3.7069 & 3.4931 & -1.4308 \\
\hline C & 3.4813 & 3.3915 & 3.1329 & -0.5334 & 3.9788 & -3.2885 & -4.3974 & 3.4046 & -2.7853 \\
\hline $\mathrm{C}$ & 3.2884 & 6.7627 & -2.5977 & 2.8596 & 7.6169 & 1.1397 & -3.5981 & 5.1325 & 3.5505 \\
\hline $\mathrm{C}$ & 1.7865 & 1.9329 & 3.8571 & -0.5724 & 1.6329 & -3.2817 & -2.6131 & 3.0476 & -4.2959 \\
\hline $\mathrm{O}$ & 2.4756 & 6.0707 & -1.6594 & 2.8633 & 6.3740 & 0.4510 & -2.8749 & 5.0286 & 2.3312 \\
\hline $\mathrm{O}$ & 4.8219 & 4.3403 & -1.5388 & -0.0369 & 6.6966 & 0.8138 & -5.0772 & 3.0766 & 2.0530 \\
\hline $\mathrm{O}$ & 4.5812 & 2.3809 & 0.4923 & -1.4738 & 4.6214 & -0.5163 & -4.8243 & 1.6101 & -0.3818 \\
\hline $\mathrm{O}$ & 2.5872 & 5.1387 & 1.8182 & 1.5117 & 4.9466 & -2.5705 & -3.3860 & 4.8647 & -1.2239 \\
\hline $\mathrm{O}$ & 2.3714 & 3.2105 & 3.9867 & -0.0967 & 2.7959 & -3.9247 & -3.6316 & 3.9159 & -3.8506 \\
\hline $\mathrm{H}$ & 1.7020 & 6.6402 & 0.8322 & 3.3292 & 5.6327 & -2.0644 & -2.5140 & 6.1875 & -0.0047 \\
\hline $\mathrm{H}$ & 3.9129 & 6.3769 & -0.2009 & 1.7094 & 7.2021 & -1.0580 & -4.5312 & 5.3956 & 1.1533 \\
\hline $\mathrm{H}$ & 2.9620 & 3.5956 & -1.0043 & 0.7929 & 4.8094 & 0.5907 & -3.2047 & 2.6506 & 1.2512 \\
\hline $\mathrm{H}$ & 5.0607 & 4.3161 & 1.0852 & -0.7211 & 6.1793 & -1.6719 & -5.6038 & 3.5319 & -0.3991 \\
\hline $\mathrm{H}$ & 2.2516 & 3.1397 & 1.3800 & 0.5756 & 3.4177 & -1.5296 & -2.7893 & 2.8859 & -1.4135 \\
\hline $\mathrm{H}$ & 4.0868 & 4.1993 & 3.5573 & -0.4526 & 4.7900 & -4.0188 & -5.3070 & 4.0145 & -2.7446 \\
\hline $\mathrm{H}$ & 4.0969 & 2.4812 & 3.0858 & -1.5857 & 3.8921 & -2.9795 & -4.6810 & 2.3570 & -2.9707 \\
\hline
\end{tabular}




\begin{tabular}{|c|c|c|c|c|c|c|c|c|c|}
\hline $\mathrm{H}$ & 2.6315 & 7.0600 & -3.4160 & 3.6991 & 7.5885 & 1.8355 & -2.9024 & 5.5421 & 4.2847 \\
\hline $\mathrm{H}$ & 3.7213 & 7.6595 & -2.1393 & 3.0041 & 8.4449 & 0.4354 & -4.4470 & 5.8163 & 3.4339 \\
\hline $\mathrm{H}$ & 4.0889 & 6.1218 & -2.9729 & 1.9245 & 7.7616 & 1.6845 & -3.9627 & 4.1572 & 3.8790 \\
\hline $\mathrm{H}$ & 0.9685 & 1.8718 & 4.5771 & -0.2040 & 0.7701 & -3.8402 & -2.2329 & 3.4492 & -5.2367 \\
\hline $\mathrm{H}$ & 1.3822 & 1.7563 & 2.8508 & -0.2251 & 1.5531 & -2.2432 & -1.7818 & 2.9896 & -3.5831 \\
\hline $\mathrm{H}$ & 2.5203 & 1.1450 & 4.0787 & -1.6734 & 1.6100 & -3.2769 & -3.0006 & 2.0334 & -4.4686 \\
\hline $\mathrm{H}$ & 5.2483 & 3.4629 & -1.5648 & -0.8269 & 6.2921 & 1.2209 & -5.4881 & 2.2129 & 1.8693 \\
\hline $\mathrm{C}$ & 5.8670 & 1.9468 & 0.8435 & -2.7194 & 5.2593 & -0.5164 & -6.0165 & 1.1019 & -0.9280 \\
\hline $\mathrm{C}$ & 6.4208 & 1.1623 & -0.3484 & -3.3276 & 5.0891 & 0.8825 & -6.7125 & 0.2765 & 0.1593 \\
\hline $\mathrm{C}$ & 5.5259 & -0.0405 & -0.5986 & -3.6515 & 3.6238 & 1.1304 & -5.8004 & -0.8759 & 0.5392 \\
\hline $\mathrm{C}$ & 5.4336 & -0.8606 & 0.6797 & -4.5548 & 3.1511 & 0.0016 & -5.4258 & -1.6646 & -0.7068 \\
\hline $\mathrm{C}$ & 5.0042 & -0.0170 & 1.8799 & -3.8318 & 3.3312 & -1.3305 & -4.8393 & -0.7681 & -1.7947 \\
\hline $\mathrm{C}$ & 5.0969 & -0.8030 & 3.1812 & -4.6446 & 2.8178 & -2.4973 & -4.5897 & -1.5175 & -3.0961 \\
\hline $\mathrm{C}$ & 7.5048 & 1.7952 & -2.3817 & -2.9913 & 6.2013 & 2.9684 & -8.0494 & 0.8150 & 2.0662 \\
\hline $\mathrm{C}$ & 7.4467 & -0.8094 & 3.5495 & -6.7946 & 2.6901 & -3.3753 & -6.8044 & -1.7575 & -3.9189 \\
\hline $\mathrm{O}$ & 6.4600 & 2.0466 & -1.4497 & -2.4006 & 5.5930 & 1.8247 & -6.9423 & 1.1536 & 1.2412 \\
\hline $\mathrm{O}$ & 6.0507 & -0.8141 & -1.6531 & -4.2567 & 3.4831 & 2.3916 & -6.4242 & -1.7068 & 1.4892 \\
\hline $\mathrm{O}$ & 4.4763 & -1.8796 & 0.4347 & -4.8470 & 1.7895 & 0.2425 & -4.4675 & -2.6200 & -0.2841 \\
\hline $\mathrm{O}$ & 5.8393 & 1.1403 & 1.9936 & -3.5636 & 4.7238 & -1.5086 & -5.7389 & 0.3146 & -2.0546 \\
\hline $\mathrm{O}$ & 6.2756 & -1.5672 & 3.3092 & -5.9662 & 3.2883 & -2.3964 & -5.6286 & -2.3988 & -3.4612 \\
\hline $\mathrm{H}$ & 6.5161 & 2.7964 & 1.0789 & -2.6119 & 6.3231 & -0.7515 & -6.6671 & 1.9146 & -1.2672 \\
\hline $\mathrm{H}$ & 7.4381 & 0.8246 & -0.1019 & -4.2587 & 5.6724 & 0.9251 & -7.6662 & -0.1042 & -0.2351 \\
\hline $\mathrm{H}$ & 4.5195 & 0.3252 & -0.8472 & -2.7104 & 3.0499 & 1.0817 & -4.8820 & -0.4316 & 0.9468 \\
\hline $\mathrm{H}$ & 6.4156 & -1.3068 & 0.8866 & -5.4791 & 3.7422 & -0.0105 & -6.3204 & -2.1705 & -1.0942 \\
\hline $\mathrm{H}$ & 3.9602 & 0.3019 & 1.7336 & -2.8825 & 2.7724 & -1.3023 & -3.8792 & -0.3658 & -1.4385 \\
\hline $\mathrm{H}$ & 4.2775 & -1.5248 & 3.2187 & -4.6387 & 1.7176 & -2.4685 & -3.6993 & -2.1416 & -2.9829 \\
\hline $\mathrm{H}$ & 4.9944 & -0.0986 & 4.0222 & -4.1775 & 3.1402 & -3.4419 & -4.4067 & -0.7761 & -3.8900 \\
\hline $\mathrm{H}$ & 7.4248 & 2.5616 & -3.1535 & -2.1707 & 6.5763 & 3.5821 & -8.1225 & 1.5972 & 2.8227 \\
\hline $\mathrm{H}$ & 8.4817 & 1.8755 & -1.8912 & -3.6310 & 7.0402 & 2.6710 & -8.9731 & 0.7969 & 1.4761 \\
\hline $\mathrm{H}$ & 7.3967 & 0.8032 & -2.8263 & -3.5768 & 5.4723 & 3.5318 & -7.9012 & -0.1559 & 2.5434 \\
\hline $\mathrm{H}$ & 8.2194 & -1.5095 & 3.8721 & -7.8143 & 3.0323 & -3.1925 & -7.4294 & -2.5254 & -4.3779 \\
\hline $\mathrm{H}$ & 7.7869 & -0.2824 & 2.6513 & -6.4897 & 2.9955 & -4.3858 & -7.3573 & -1.2807 & -3.1020 \\
\hline $\mathrm{H}$ & 7.2783 & -0.0667 & 4.3402 & -6.7477 & 1.5969 & -3.2992 & -6.5668 & -0.9896 & -4.6667 \\
\hline $\mathrm{H}$ & 5.5827 & -1.6710 & -1.6452 & -4.7760 & 2.6569 & 2.3935 & -5.8834 & -2.5146 & 1.5717 \\
\hline $\mathrm{C}$ & 4.8273 & -3.2053 & 0.7250 & -6.1784 & 1.3471 & 0.1200 & -4.6379 & -3.9524 & -0.6861 \\
\hline $\mathrm{C}$ & 4.4100 & -4.0651 & -0.4779 & -6.5014 & 0.5955 & 1.4132 & -4.3902 & -4.8276 & 0.5468 \\
\hline $\mathrm{C}$ & 2.8931 & -4.1889 & -0.5559 & -5.5568 & -0.5883 & 1.5332 & -2.9273 & -4.7453 & 0.9559 \\
\hline $\mathrm{C}$ & 2.3381 & -4.6348 & 0.7961 & -5.5527 & -1.4141 & 0.2476 & -2.0331 & -5.0144 & -0.2513 \\
\hline $\mathrm{C}$ & 2.7866 & -3.6233 & 1.8541 & -5.4321 & -0.5800 & -1.0276 & -2.3878 & -4.1056 & -1.4235 \\
\hline $\mathrm{C}$ & 2.2697 & -3.9121 & 3.2504 & -5.7857 & -1.4274 & -2.2381 & -1.6134 & -4.4569 & -2.6802 \\
\hline $\mathrm{C}$ & 5.3100 & -4.3293 & -2.6834 & -7.0649 & 1.2497 & 3.6450 & -5.6972 & -5.3574 & 2.4730 \\
\hline $\mathrm{C}$ & 1.7386 & -1.7388 & 4.0081 & -4.2558 & -0.7810 & -3.9144 & -0.9161 & -2.4031 & -3.5862 \\
\hline $\mathrm{O}$ & 4.9340 & -3.4428 & -1.6367 & -6.3304 & 1.5288 & 2.4605 & -5.2585 & -4.3612 & 1.5595 \\
\hline $\mathrm{O}$ & 2.5588 & -5.0726 & -1.5898 & -5.9270 & -1.4023 & 2.6233 & -2.6704 & -5.6841 & 1.9730 \\
\hline $\mathrm{O}$ & 0.9284 & -4.6935 & 0.6697 & -4.4288 & -2.2740 & 0.3116 & -0.6957 & -4.7936 & 0.1728 \\
\hline $\mathrm{O}$ & 4.2133 & -3.6504 & 1.9051 & -6.3383 & 0.5175 & -0.9961 & -3.7691 & -4.2886 & -1.7316 \\
\hline $\mathrm{O}$ & 2.5642 & -2.8778 & 4.1682 & -5.5971 & -0.7680 & -3.4696 & -1.7994 & -3.5055 & -3.7062 \\
\hline $\mathrm{H}$ & 5.9049 & -3.2858 & 0.8974 & -6.8534 & 2.1950 & -0.0246 & -5.6500 & -4.1102 & -1.0707 \\
\hline $\mathrm{H}$ & 4.8473 & -5.0667 & -0.3562 & -7.5441 & 0.2484 & 1.3753 & -4.6325 & -5.8684 & 0.2871 \\
\hline $\mathrm{H}$ & 2.5045 & -3.1765 & -0.7524 & -4.5402 & -0.1910 & 1.6695 & -2.7264 & -3.7183 & 1.3012 \\
\hline $\mathrm{H}$ & 2.7383 & -5.6285 & 1.0448 & -6.4855 & -1.9976 & 0.2103 & -2.1652 & -6.0611 & -0.5633 \\
\hline $\mathrm{H}$ & 2.4473 & -2.6252 & 1.5395 & -4.4000 & -0.2098 & -1.1002 & -2.2014 & -3.0588 & -1.1383 \\
\hline $\mathrm{H}$ & 2.7530 & -4.8192 & 3.6301 & -6.8502 & -1.6807 & -2.1770 & -1.9885 & -5.4165 & -3.0549 \\
\hline $\mathrm{H}$ & 1.1865 & -4.0791 & 3.2019 & -5.1971 & -2.3563 & -2.1978 & -0.5493 & -4.5688 & -2.4413 \\
\hline $\mathrm{H}$ & 5.7203 & -3.7084 & -3.4816 & -6.8880 & 2.0844 & 4.3242 & -6.3798 & -4.8661 & 3.1672 \\
\hline $\mathrm{H}$ & 6.0799 & -5.0288 & -2.3373 & -8.1368 & 1.1873 & 3.4230 & -6.2337 & -6.1543 & 1.9444 \\
\hline
\end{tabular}




\begin{tabular}{|c|c|c|c|c|c|c|c|c|c|}
\hline $\mathrm{H}$ & 4.4435 & -4.8848 & -3.0485 & -6.7335 & 0.3152 & 4.1016 & -4.8533 & -5.7857 & 3.0180 \\
\hline $\mathrm{H}$ & 1.9019 & -1.1027 & 4.8810 & -4.2544 & -0.4407 & -4.9514 & -1.1668 & -1.7044 & -4.3876 \\
\hline $\mathrm{H}$ & 1.9965 & -1.1639 & 3.1065 & -3.6236 & -0.0972 & -3.3313 & -1.0238 & -1.8867 & -2.6219 \\
\hline $\mathrm{H}$ & 0.6802 & -2.0230 & 3.9528 & -3.8371 & -1.7947 & -3.8572 & 0.1246 & -2.7343 & -3.6929 \\
\hline $\mathrm{H}$ & 1.6226 & -4.9284 & -1.8465 & -5.1857 & -2.0191 & 2.7743 & -1.7264 & -5.6128 & 2.2081 \\
\hline $\mathrm{C}$ & 0.2565 & -5.7654 & 1.2813 & -4.6405 & -3.6242 & 0.6003 & 0.1725 & -5.8915 & 0.1592 \\
\hline $\mathrm{C}$ & -0.6579 & -6.4218 & 0.2488 & -3.6019 & -4.0370 & 1.6469 & 1.0261 & -5.8351 & 1.4277 \\
\hline $\mathrm{C}$ & -1.6781 & -5.3936 & -0.2053 & -2.1961 & -3.9531 & 1.0521 & 1.9724 & -4.6457 & 1.3725 \\
\hline $\mathrm{C}$ & -2.3883 & -4.7424 & 0.9794 & -2.1631 & -4.8106 & -0.2182 & 2.7523 & -4.6642 & 0.0605 \\
\hline $\mathrm{C}$ & -1.4196 & -4.2775 & 2.0629 & -3.2328 & -4.2837 & -1.1818 & 1.8006 & -4.7332 & -1.1332 \\
\hline $\mathrm{C}$ & -2.1443 & -3.8931 & 3.3391 & -3.2736 & -5.0193 & -2.5069 & 2.5225 & -4.8425 & -2.4641 \\
\hline $\mathrm{C}$ & -0.3214 & -8.0536 & -1.4350 & -3.4783 & -3.7688 & 4.0178 & 0.6096 & -6.3749 & 3.7174 \\
\hline $\mathrm{C}$ & -0.7304 & -4.1273 & 5.2312 & -5.4059 & -4.7260 & -3.5157 & 1.1312 & -5.8790 & -4.0901 \\
\hline $\mathrm{O}$ & 0.1461 & -6.8669 & -0.8176 & -3.7437 & -3.1658 & 2.7560 & 0.1396 & -5.7564 & 2.5255 \\
\hline $\mathrm{O}$ & -2.6180 & -6.0009 & -1.0646 & -1.2199 & -4.3050 & 2.0041 & 2.8443 & -4.6949 & 2.4741 \\
\hline $\mathrm{O}$ & -3.0945 & -3.6083 & 0.4948 & -0.8903 & -4.7065 & -0.8308 & 3.5301 & -3.4737 & 0.0436 \\
\hline $\mathrm{O}$ & -0.5116 & -5.3302 & 2.3789 & -4.5146 & -4.4051 & -0.5623 & 0.9799 & -5.8944 & -0.9912 \\
\hline $\mathrm{O}$ & -1.2807 & -3.2570 & 4.2567 & -4.0490 & -4.3223 & -3.4615 & 1.6429 & -4.6710 & -3.5556 \\
\hline $\mathrm{H}$ & 0.9714 & -6.4932 & 1.6777 & -5.6547 & -3.7923 & 0.9774 & -0.3915 & -6.8295 & 0.1338 \\
\hline $\mathrm{H}$ & -1.1659 & -7.2673 & 0.7366 & -3.8123 & -5.0725 & 1.9530 & 1.6179 & -6.7602 & 1.4820 \\
\hline $\mathrm{H}$ & -1.1124 & -4.6086 & -0.7268 & -2.0102 & -2.9091 & 0.7651 & 1.3589 & -3.7287 & 1.3906 \\
\hline $\mathrm{H}$ & -3.0877 & -5.4720 & 1.4152 & -2.3839 & -5.8579 & 0.0347 & 3.4111 & -5.5451 & 0.0543 \\
\hline $\mathrm{H}$ & -0.8612 & -3.4031 & 1.7028 & -3.0176 & -3.2244 & -1.3885 & 1.1724 & -3.8290 & -1.1440 \\
\hline $\mathrm{H}$ & -2.6038 & -4.7872 & 3.7837 & -3.6514 & -6.0417 & -2.3605 & 3.0374 & -5.8130 & -2.5193 \\
\hline $\mathrm{H}$ & -2.9432 & -3.1904 & 3.0770 & -2.2515 & -5.0727 & -2.8959 & 3.2742 & -4.0497 & -2.5174 \\
\hline $\mathrm{H}$ & 0.3990 & -8.3088 & -2.2140 & -3.6441 & -2.9983 & 4.7720 & -0.1935 & -6.2889 & 4.4499 \\
\hline $\mathrm{H}$ & -0.3703 & -8.8776 & -0.7112 & -4.1641 & -4.6050 & 4.1986 & 0.8262 & -7.4352 & 3.5418 \\
\hline $\mathrm{H}$ & -1.3094 & -7.9072 & -1.8813 & -2.4467 & -4.1217 & 4.0637 & 1.5072 & -5.8742 & 4.0855 \\
\hline $\mathrm{H}$ & 0.0241 & -3.5569 & 5.7779 & -5.8975 & -4.0795 & -4.2459 & 0.4230 & -5.6005 & -4.8734 \\
\hline $\mathrm{H}$ & -1.5041 & -4.4661 & 5.9317 & -5.4890 & -5.7695 & -3.8460 & 1.9361 & -6.4823 & -4.5291 \\
\hline $\mathrm{H}$ & -0.2564 & -4.9993 & 4.7701 & -5.8994 & -4.6169 & -2.5450 & 0.6136 & -6.4701 & -3.3282 \\
\hline $\mathrm{H}$ & -3.1095 & -5.2801 & -1.5042 & -0.9562 & -5.2338 & 1.8610 & 3.6186 & -4.1343 & 2.2774 \\
\hline $\mathrm{C}$ & -4.4665 & -3.7717 & 0.2666 & -0.1851 & -5.9008 & -1.1021 & 4.8864 & -3.5678 & -0.3208 \\
\hline $\mathrm{C}$ & -4.8128 & -3.1630 & -1.1022 & 0.6312 & -6.3495 & 0.1186 & 5.7201 & -2.8778 & 0.7643 \\
\hline $\mathrm{C}$ & -4.7446 & -1.6406 & -1.0787 & 1.5522 & -5.2015 & 0.5041 & 5.4986 & -1.3722 & 0.7534 \\
\hline $\mathrm{C}$ & -5.5074 & -1.1190 & 0.1351 & 2.4174 & -4.8655 & -0.7025 & 5.6267 & -0.8329 & -0.6663 \\
\hline $\mathrm{C}$ & -5.0032 & -1.7759 & 1.4136 & 1.5692 & -4.5615 & -1.9366 & 4.7545 & -1.6097 & -1.6449 \\
\hline $\mathrm{C}$ & -5.7005 & -1.2501 & 2.6590 & 2.4087 & -4.3404 & -3.1743 & 4.9116 & -1.1042 & -3.0730 \\
\hline $\mathrm{C}$ & -4.2399 & -3.6083 & -3.4004 & 0.2118 & -7.7027 & 2.0424 & 6.3067 & -3.4070 & 3.0311 \\
\hline $\mathrm{C}$ & -7.8058 & -2.3418 & 2.5125 & 4.3368 & -5.0343 & -4.2784 & 7.0486 & -1.9660 & -3.6366 \\
\hline $\mathrm{O}$ & -3.9089 & -3.7457 & -2.0233 & -0.2766 & -6.7076 & 1.1462 & 5.3409 & -3.4726 & 1.9888 \\
\hline $\mathrm{O}$ & -5.3094 & -1.1355 & -2.2682 & 2.3494 & -5.5431 & 1.6148 & 6.4404 & -0.7696 & 1.6074 \\
\hline $\mathrm{O}$ & -5.2849 & 0.2771 & 0.1640 & 3.1895 & -3.7292 & -0.3411 & 5.2153 & 0.5215 & -0.6259 \\
\hline $\mathrm{O}$ & -5.2116 & -3.1865 & 1.3034 & 0.6722 & -5.6519 & -2.1766 & 5.1161 & -2.9922 & -1.5800 \\
\hline $\mathrm{O}$ & -7.0988 & -1.1147 & 2.5284 & 3.4068 & -5.3331 & -3.2579 & 6.2426 & -0.8181 & -3.4385 \\
\hline $\mathrm{H}$ & -4.7317 & -4.8338 & 0.2665 & -0.8732 & -6.6961 & -1.4138 & 5.1841 & -4.6175 & -0.4074 \\
\hline $\mathrm{H}$ & -5.8443 & -3.4541 & -1.3541 & 1.2204 & -7.2315 & -0.1706 & 6.7821 & -3.0790 & 0.5593 \\
\hline $\mathrm{H}$ & -3.6891 & -1.3372 & -0.9745 & 0.9177 & -4.3351 & 0.7290 & 4.4716 & -1.1801 & 1.0999 \\
\hline $\mathrm{H}$ & -6.5778 & -1.3340 & 0.0078 & 3.0767 & -5.7144 & -0.9200 & 6.6768 & -0.9044 & -0.9797 \\
\hline $\mathrm{H}$ & -3.9267 & -1.5706 & 1.5136 & 0.9869 & -3.6520 & -1.7485 & 3.6971 & -1.5029 & -1.3593 \\
\hline $\mathrm{H}$ & -5.3200 & -0.2437 & 2.8614 & 2.8739 & -3.3489 & -3.1189 & 4.3701 & -0.1572 & -3.1625 \\
\hline $\mathrm{H}$ & -5.4494 & -1.9053 & 3.5067 & 1.7542 & -4.3650 & -4.0598 & 4.4633 & -1.8382 & -3.7596 \\
\hline $\mathrm{H}$ & -3.5776 & -4.2858 & -3.9428 & -0.5791 & -7.8808 & 2.7712 & 5.9427 & -4.0549 & 3.8302 \\
\hline $\mathrm{H}$ & -5.2829 & -3.8936 & -3.5786 & 0.4176 & -8.6297 & 1.4962 & 7.2753 & -3.7790 & 2.6784 \\
\hline $\mathrm{H}$ & -4.0850 & -2.5821 & -3.7381 & 1.1184 & -7.3605 & 2.5446 & 6.4214 & -2.3849 & 3.3969 \\
\hline $\mathrm{H}$ & -8.8636 & -2.1016 & 2.6303 & 5.1051 & -5.8090 & -4.2588 & 7.9704 & -1.6302 & -4.1141 \\
\hline
\end{tabular}




\begin{tabular}{|c|c|c|c|c|c|c|c|c|c|}
\hline $\mathrm{H}$ & -7.6573 & -2.8871 & 1.5748 & 3.8542 & -5.0336 & -5.2656 & 7.2892 & -2.4655 & -2.6919 \\
\hline $\mathrm{H}$ & -7.4867 & -2.9866 & 3.3417 & 4.7945 & -4.0529 & -4.1046 & 6.5443 & -2.6890 & -4.2909 \\
\hline $\mathrm{H}$ & -5.6246 & -0.2300 & -2.0936 & 3.0656 & -4.8819 & 1.6702 & 6.4414 & 0.1891 & 1.4284 \\
\hline $\mathrm{C}$ & -6.3459 & 1.1393 & 0.5038 & 4.5685 & -3.7649 & -0.5938 & 6.0166 & 1.4989 & -1.2464 \\
\hline $\mathrm{C}$ & -6.5727 & 2.0542 & -0.6986 & 5.2840 & -3.2325 & 0.6572 & 6.3325 & 2.5489 & -0.1807 \\
\hline $\mathrm{C}$ & -5.2968 & 2.8365 & -0.9701 & 5.0735 & -1.7316 & 0.8076 & 5.0246 & 3.1388 & 0.3199 \\
\hline $\mathrm{C}$ & -4.7392 & 3.4731 & 0.3048 & 5.3057 & -1.0038 & -0.5136 & 4.1760 & 3.6261 & -0.8530 \\
\hline $\mathrm{C}$ & -4.7449 & 2.5354 & 1.5133 & 4.5132 & -1.6441 & -1.6461 & 4.0817 & 2.6388 & -2.0169 \\
\hline $\mathrm{C}$ & -4.4655 & 3.2787 & 2.8070 & 4.8016 & -1.0133 & -2.9971 & 3.5990 & 3.3701 & -3.2612 \\
\hline $\mathrm{C}$ & -7.6705 & 1.8000 & -2.8119 & 5.4484 & -3.8342 & 2.9780 & 7.9397 & 2.6936 & 1.5860 \\
\hline $\mathrm{C}$ & -2.6776 & 2.1124 & 3.7879 & 2.6494 & -0.7032 & -3.9099 & 1.8489 & 2.1243 & -4.1844 \\
\hline $\mathrm{O}$ & -6.9056 & 1.1995 & -1.7756 & 4.7713 & -3.9856 & 1.7365 & 7.0400 & 1.8810 & 0.8441 \\
\hline $\mathrm{O}$ & -5.5452 & 3.8316 & -1.9381 & 5.9546 & -1.2269 & 1.7825 & 5.2873 & 4.2048 & 1.2056 \\
\hline $\mathrm{O}$ & -3.3880 & 3.8391 & 0.0379 & 4.8862 & 0.3351 & -0.2926 & 2.8510 & 3.8465 & -0.3883 \\
\hline $\mathrm{O}$ & -6.0137 & 1.8978 & 1.6369 & 4.8988 & -3.0154 & -1.7316 & 5.3550 & 2.0833 & -2.3332 \\
\hline $\mathrm{O}$ & -4.0575 & 2.4064 & 3.8394 & 3.8761 & -1.3985 & -3.9909 & 3.2037 & 2.5010 & -4.2968 \\
\hline $\mathrm{H}$ & -7.2443 & 0.5712 & 0.7563 & 4.8893 & -4.7893 & -0.8057 & 6.9293 & 1.0499 & -1.6474 \\
\hline $\mathrm{H}$ & -7.4029 & 2.7412 & -0.4812 & 6.3632 & -3.4214 & 0.5450 & 6.9589 & 3.3345 & -0.6270 \\
\hline $\mathrm{H}$ & -4.5440 & 2.1169 & -1.3258 & 4.0225 & -1.5617 & 1.0903 & 4.4672 & 2.3378 & 0.8255 \\
\hline $\mathrm{H}$ & -5.3378 & 4.3663 & 0.5387 & 6.3774 & -1.0359 & -0.7587 & 4.6128 & 4.5683 & -1.2189 \\
\hline $\mathrm{H}$ & -3.9848 & 1.7626 & 1.3707 & 3.4409 & -1.5719 & -1.4118 & 3.3866 & 1.8339 & -1.7385 \\
\hline $\mathrm{H}$ & -5.3866 & 3.7698 & 3.1368 & 5.7873 & -1.3535 & -3.3311 & 4.4336 & 3.9714 & -3.6374 \\
\hline $\mathrm{H}$ & -3.7039 & 4.0516 & 2.6200 & 4.8299 & 0.0794 & -2.8912 & 2.7799 & 4.0490 & -2.9787 \\
\hline $\mathrm{H}$ & -7.9036 & 1.0085 & -3.5250 & 5.1184 & -4.6612 & 3.6104 & 8.4411 & 2.0348 & 2.2960 \\
\hline $\mathrm{H}$ & -8.6051 & 2.2076 & -2.4085 & 6.5335 & -3.8963 & 2.8363 & 8.6873 & 3.1405 & 0.9201 \\
\hline $\mathrm{H}$ & -7.1104 & 2.5973 & -3.3043 & 5.1976 & -2.8785 & 3.4442 & 7.4060 & 3.4834 & 2.1180 \\
\hline $\mathrm{H}$ & -2.4632 & 1.4023 & 4.5878 & 2.0243 & -1.0548 & -4.7324 & 1.6434 & 1.3820 & -4.9576 \\
\hline $\mathrm{H}$ & -2.3839 & 1.6601 & 2.8300 & 2.1211 & -0.8910 & -2.9644 & 1.6364 & 1.6847 & -3.2035 \\
\hline $\mathrm{H}$ & -2.0752 & 3.0194 & 3.9385 & 2.8021 & 0.3808 & -4.0117 & 1.1828 & 2.9887 & -4.3231 \\
\hline $\mathrm{H}$ & -4.6731 & 4.1417 & -2.2459 & 5.7815 & -0.2704 & 1.8637 & 4.4605 & 4.3593 & 1.6997 \\
\hline $\mathrm{H}$ & -1.4250 & 5.7111 & 3.2277 & 4.5643 & 2.3177 & -4.0019 & 0.2758 & 5.8020 & -3.1471 \\
\hline $\mathrm{C}$ & 3.2072 & -0.1247 & -3.2686 & 0.3944 & -1.5618 & 0.0985 & 2.4037 & -1.4066 & 2.7861 \\
\hline $\mathrm{C}$ & -0.7681 & -1.1559 & -0.3752 & 1.9827 & 2.4796 & 2.9729 & -2.7339 & -0.2147 & 2.4480 \\
\hline $\mathrm{C}$ & 1.5714 & 2.1099 & -3.5516 & -1.8828 & 0.0358 & 0.2800 & 1.8843 & 0.1571 & 5.0218 \\
\hline $\mathrm{C}$ & 3.7610 & 1.1565 & -3.2493 & -0.4436 & -1.4083 & -1.0072 & 3.0237 & -1.6991 & 4.0010 \\
\hline $\mathrm{C}$ & -1.6964 & 1.1593 & -1.5731 & -0.7255 & 2.9944 & 3.0558 & -2.0771 & 1.7180 & 4.2965 \\
\hline $\mathrm{C}$ & -1.4758 & -0.2319 & 0.3865 & 1.5401 & 3.7926 & 3.0965 & -3.7193 & 0.2712 & 3.2986 \\
\hline $\mathrm{C}$ & 2.9484 & 2.2775 & -3.3855 & -1.5827 & -0.6160 & -0.9175 & 2.7605 & -0.9245 & 5.1246 \\
\hline $\mathrm{C}$ & -1.9336 & 0.9351 & -0.2200 & 0.1740 & 4.0516 & 3.1475 & -3.3905 & 1.2661 & 4.2166 \\
\hline $\mathrm{C}$ & -0.5004 & -5.4135 & -3.7071 & 3.6019 & -1.7356 & 5.6233 & -0.8588 & 0.8377 & -2.2626 \\
\hline $\mathrm{C}$ & 2.0938 & -2.6899 & -3.8657 & 1.5310 & -2.3925 & 2.5819 & 1.6026 & -0.4040 & 0.2609 \\
\hline $\mathrm{C}$ & -0.5618 & -3.1186 & -2.9708 & 2.3131 & -0.2601 & 4.2531 & -1.0381 & 0.5547 & 0.0869 \\
\hline $\mathrm{C}$ & 1.2645 & -3.8951 & -4.2923 & 2.0979 & -2.6058 & 3.9714 & 1.0758 & 0.3173 & -0.9653 \\
\hline $\mathrm{C}$ & -0.4805 & 0.6248 & -3.7163 & -1.3270 & 0.5845 & 2.7006 & 0.3603 & 1.6627 & 3.6588 \\
\hline $\mathrm{C}$ & 1.8316 & -0.2969 & -3.4419 & 0.0869 & -0.9256 & 1.3053 & 1.5211 & -0.3272 & 2.6835 \\
\hline $\mathrm{C}$ & -0.4909 & -0.9227 & -1.7254 & 1.0901 & 1.4040 & 2.8892 & -1.4224 & 0.2735 & 2.4824 \\
\hline $\mathrm{C}$ & 1.0099 & 0.8385 & -3.6044 & -1.0619 & -0.1170 & 1.3937 & 1.2704 & 0.4728 & 3.8137 \\
\hline $\mathrm{C}$ & -0.9618 & 0.2485 & -2.3337 & -0.2906 & 1.6711 & 2.9186 & -1.0757 & 1.2284 & 3.4490 \\
\hline $\mathrm{C}$ & 0.3193 & -1.9907 & -2.4291 & 1.7339 & 0.0187 & 2.8577 & -0.5351 & -0.1833 & 1.3359 \\
\hline $\mathrm{N}$ & 0.2878 & -4.2695 & -3.2675 & 3.0184 & -1.5304 & 4.3080 & -0.3559 & 0.1081 & -1.1155 \\
\hline $\mathrm{N}$ & 1.2178 & -1.5713 & -3.5223 & 0.8532 & -1.0985 & 2.4904 & 0.9113 & 0.0590 & 1.4588 \\
\hline $\mathrm{H}$ & 3.8543 & -0.9842 & -3.1184 & 1.2865 & -2.1785 & 0.0210 & 2.6274 & -2.0196 & 1.9189 \\
\hline $\mathrm{H}$ & -0.3807 & -2.0641 & 0.0839 & 3.0530 & 2.2869 & 2.9291 & -2.9840 & -0.9832 & 1.7172 \\
\hline $\mathrm{H}$ & 0.9220 & 2.9797 & -3.6298 & -2.7760 & 0.6539 & 0.3553 & 1.6883 & 0.7819 & 5.8891 \\
\hline $\mathrm{H}$ & 4.8294 & 1.2655 & -3.0880 & -0.2112 & -1.9124 & -1.9410 & 3.7024 & -2.5446 & 4.0569 \\
\hline $\mathrm{H}$ & -2.0530 & 2.0807 & -2.0312 & -1.7942 & 3.1982 & 3.0795 & -1.8093 & 2.4653 & 5.0393 \\
\hline
\end{tabular}




\begin{tabular}{rrrrrrrrrr}
$\mathrm{H}$ & -1.6423 & -0.4134 & 1.4452 & 2.2624 & 4.6010 & 3.1455 & -4.7312 & -0.1218 & 3.2352 \\
$\mathrm{H}$ & 3.3803 & 3.2735 & -3.3394 & -2.2374 & -0.5097 & -1.7772 & 3.2389 & -1.1494 & 6.0721 \\
$\mathrm{H}$ & -2.4370 & 1.7068 & 0.3470 & -0.1921 & 5.0709 & 3.2227 & -4.1467 & 1.6733 & 4.8800 \\
$\mathrm{H}$ & 0.1637 & -6.2663 & -3.8764 & 4.1344 & -2.6910 & 5.6417 & -0.3826 & 0.4821 & -3.1817 \\
$\mathrm{H}$ & -1.2188 & -5.6862 & -2.9299 & 4.3157 & -0.9361 & 5.8406 & -1.9410 & 0.6916 & -2.3565 \\
$\mathrm{H}$ & -1.0411 & -5.2021 & -4.6459 & 2.8402 & -1.7490 & 6.4230 & -0.6626 & 1.9230 & -2.1638 \\
$\mathrm{H}$ & 2.7359 & -2.3884 & -4.6981 & 0.7864 & -3.1638 & 2.3698 & 2.6692 & -0.1788 & 0.3673 \\
$\mathrm{H}$ & 2.7414 & -2.9803 & -3.0228 & 2.3554 & -2.4959 & 1.8566 & 1.4944 & -1.4942 & 0.1101 \\
$\mathrm{H}$ & -1.3182 & -3.3988 & -2.2313 & 3.0121 & 0.5408 & 4.5131 & -2.1135 & 0.3780 & -0.0308 \\
$\mathrm{H}$ & -1.0930 & -2.7594 & -3.8728 & 1.4766 & -0.2284 & 4.9801 & -0.8996 & 1.6442 & 0.2532 \\
$\mathrm{H}$ & 0.7500 & -3.6628 & -5.2420 & 1.2714 & -2.6659 & 4.7058 & 1.3084 & 1.3986 & -0.8697 \\
$\mathrm{H}$ & 1.9319 & -4.7461 & -4.4639 & 2.6369 & -3.5603 & 3.9879 & 1.5873 & -0.0671 & -1.8569 \\
$\mathrm{H}$ & -0.9772 & 1.5390 & -4.0517 & -2.3242 & 1.0379 & 2.6917 & 0.4148 & 2.2958 & 4.5483 \\
$\mathrm{H}$ & -0.6839 & -0.1750 & -4.4363 & -1.2867 & -0.1502 & 3.5127 & 0.7057 & 2.2626 & 2.8115 \\
$\mathrm{H}$ & 0.9320 & -2.4584 & -1.6409 & 2.5880 & 0.0657 & 2.1527 & -0.7081 & -1.2654 & 1.1747 \\
\hline
\end{tabular}

\begin{tabular}{|c|c|c|c|c|c|c|}
\hline & \\
\hline atom & $\mathbf{x}$ & $\mathbf{y}$ & $\mathbf{Z}$ & $\mathbf{x}$ & $\mathbf{y}$ & $\mathbf{z}$ \\
\hline C & 5.5125 & 2.0073 & -1.2894 & 4.0198 & 4.6299 & -0.2456 \\
\hline $\mathrm{C}$ & 5.4932 & 2.9590 & -0.0880 & 3.3913 & 5.2504 & 1.0077 \\
\hline $\mathrm{C}$ & 4.0611 & 3.3680 & 0.2257 & 1.8924 & 4.9858 & 1.0386 \\
\hline $\mathrm{C}$ & 3.4241 & 3.9206 & -1.0416 & 1.2886 & 5.4285 & -0.2880 \\
\hline C & 3.5122 & 2.9204 & -2.1921 & 1.9795 & 4.7332 & -1.4568 \\
\hline $\mathrm{C}$ & 2.9401 & 3.4683 & -3.4920 & 1.4123 & 5.1467 & -2.8077 \\
\hline $\mathrm{C}$ & 6.8022 & 3.1357 & 1.9002 & 4.1543 & 5.5628 & 3.2476 \\
\hline $\mathrm{C}$ & 4.6241 & 5.0174 & -4.1310 & 2.3098 & 7.3336 & -3.0412 \\
\hline $\mathrm{O}$ & 6.1121 & 2.2870 & 0.9909 & 4.0568 & 4.6946 & 2.1243 \\
\hline $\mathrm{O}$ & 4.0605 & 4.3468 & 1.2408 & 1.3068 & 5.6838 & 2.1114 \\
\hline $\mathrm{O}$ & 2.0801 & 4.2283 & -0.7130 & -0.0888 & 5.1053 & -0.2306 \\
\hline $\mathrm{O}$ & 4.8896 & 2.5912 & -2.4065 & 3.3722 & 5.0614 & -1.4165 \\
\hline $\mathrm{O}$ & 3.2775 & 4.8148 & -3.7434 & 1.1511 & 6.5278 & -2.9215 \\
\hline $\mathrm{H}$ & 6.5457 & 1.8021 & -1.5889 & 5.0600 & 4.9586 & -0.3370 \\
\hline $\mathrm{H}$ & 6.0703 & 3.8570 & -0.3540 & 3.5580 & 6.3369 & 0.9686 \\
\hline $\mathrm{H}$ & 3.5038 & 2.4720 & 0.5435 & 1.7427 & 3.8985 & 1.1461 \\
\hline $\mathrm{H}$ & 3.9573 & 4.8375 & -1.3282 & 1.4155 & 6.5153 & -0.3892 \\
\hline $\mathrm{H}$ & 2.9562 & 2.0091 & -1.9220 & 1.8567 & 3.6436 & -1.3491 \\
\hline $\mathrm{H}$ & 1.8482 & 3.4348 & -3.4422 & 0.4495 & 4.6467 & -2.9520 \\
\hline $\mathrm{H}$ & 7.2425 & 2.4885 & 2.6605 & 4.7209 & 5.0250 & 4.0084 \\
\hline $\mathrm{H}$ & 7.6012 & 3.6826 & 1.3861 & 4.6898 & 6.4810 & 2.9794 \\
\hline $\mathrm{H}$ & 6.1141 & 3.8444 & 2.3666 & 3.1627 & 5.8156 & 3.6281 \\
\hline $\mathrm{H}$ & 4.6979 & 6.0401 & -4.5048 & 1.9760 & 8.3292 & -3.3380 \\
\hline $\mathrm{H}$ & 5.3178 & 4.8858 & -3.2935 & 2.8620 & 7.4008 & -2.0977 \\
\hline $\mathrm{H}$ & 4.9114 & 4.3194 & -4.9282 & 2.9856 & 6.9346 & -3.8086 \\
\hline $\mathrm{H}$ & 3.2030 & 4.8129 & 1.1961 & 0.3643 & 5.8205 & 1.9017 \\
\hline $\mathrm{C}$ & 1.5602 & 5.4463 & -1.1830 & -1.0094 & 6.0364 & -0.7524 \\
\hline C & 0.8759 & 6.1563 & -0.0048 & -2.0544 & 6.2992 & 0.3357 \\
\hline $\mathrm{C}$ & -0.4186 & 5.4512 & 0.3866 & -2.8506 & 5.0309 & 0.5816 \\
\hline $\mathrm{C}$ & -1.2510 & 5.0950 & -0.8442 & -3.3715 & 4.4212 & -0.7138 \\
\hline $\mathrm{C}$ & -0.4225 & 4.3838 & -1.9051 & -2.2596 & 4.2891 & -1.7494 \\
\hline C & -1.1890 & 4.1241 & -3.1897 & -2.7875 & 3.8841 & -3.1130 \\
\hline $\mathrm{C}$ & 1.5061 & 6.9454 & 2.1730 & -2.0371 & 7.5633 & 2.3680 \\
\hline $\mathrm{C}$ & -0.8549 & 1.8362 & -3.6228 & -1.4192 & 2.0947 & -3.7895 \\
\hline $\mathrm{O}$ & 1.8460 & 6.1845 & 1.0204 & -1.3344 & 6.6948 & 1.4890 \\
\hline $\mathrm{O}$ & -1.1824 & 6.2888 & 1.2253 & -3.9056 & 5.2832 & 1.4797 \\
\hline $\mathrm{O}$ & -2.3051 & 4.2649 & -0.3713 & -3.8358 & 3.1203 & -0.3755 \\
\hline $\mathrm{O}$ & 0.6703 & 5.2349 & -2.2427 & -1.6121 & 5.5480 & -1.9179 \\
\hline $\mathrm{O}$ & -0.5580 & 3.1604 & -4.0075 & -1.7610 & 3.4503 & -3.9789 \\
\hline
\end{tabular}




\begin{tabular}{|c|c|c|c|c|c|c|}
\hline $\mathrm{H}$ & 2.3624 & 6.0704 & -1.5858 & -0.4962 & 6.9576 & -1.0383 \\
\hline $\mathrm{H}$ & 0.6276 & 7.1827 & -0.3180 & -2.7175 & 7.1101 & 0.0019 \\
\hline $\mathrm{H}$ & -0.1473 & 4.5175 & 0.9028 & -2.1549 & 4.2948 & 0.9977 \\
\hline $\mathrm{H}$ & -1.6599 & 6.0210 & -1.2739 & -4.1904 & 5.0327 & -1.1202 \\
\hline $\mathrm{H}$ & -0.0420 & 3.4367 & -1.4956 & -1.5366 & 3.5449 & -1.3859 \\
\hline $\mathrm{H}$ & -1.2254 & 5.0579 & -3.7597 & -3.2517 & 4.7612 & -3.5752 \\
\hline $\mathrm{H}$ & -2.2175 & 3.8218 & -2.9560 & -3.5572 & 3.1083 & -2.9912 \\
\hline $\mathrm{H}$ & 2.4235 & 7.0380 & 2.7579 & -1.3473 & 7.8139 & 3.1748 \\
\hline $\mathrm{H}$ & 1.1550 & 7.9435 & 1.8858 & -2.3294 & 8.4818 & 1.8455 \\
\hline $\mathrm{H}$ & 0.7342 & 6.4355 & 2.7541 & -2.9254 & 7.0737 & 2.7724 \\
\hline $\mathrm{H}$ & -0.3996 & 1.1744 & -4.3613 & -0.6523 & 1.8482 & -4.5262 \\
\hline $\mathrm{H}$ & -0.4561 & 1.5860 & -2.6281 & -1.0194 & 1.8998 & -2.7838 \\
\hline $\mathrm{H}$ & -1.9411 & 1.6625 & -3.6148 & -2.2910 & 1.4429 & -3.9488 \\
\hline $\mathrm{H}$ & -2.0014 & 5.8088 & 1.4445 & -4.2752 & 4.4172 & 1.7347 \\
\hline C & -3.6254 & 4.6793 & -0.5963 & -5.2134 & 2.8918 & -0.4064 \\
\hline C & -4.4318 & 4.3691 & 0.6651 & -5.5858 & 2.0449 & 0.8205 \\
\hline C & -4.5405 & 2.8654 & 0.8749 & -5.0511 & 0.6218 & 0.6961 \\
\hline C & -5.0713 & 2.2335 & -0.4063 & -5.3770 & 0.0598 & -0.6841 \\
\hline C & -4.2129 & 2.6168 & -1.6080 & -4.9236 & 1.0023 & -1.7924 \\
\hline C & -4.7342 & 2.0220 & -2.9098 & -5.2231 & 0.4743 & -3.1875 \\
\hline C & -4.7089 & 5.5418 & 2.7141 & -5.5622 & 2.3248 & 3.2058 \\
\hline C & -6.6337 & 3.3869 & -3.3160 & -7.5570 & 0.8943 & -3.3601 \\
\hline $\mathrm{O}$ & -3.7992 & 5.0229 & 1.7491 & -5.0677 & 2.7365 & 1.9383 \\
\hline $\mathrm{O}$ & -5.4076 & 2.6140 & 1.9540 & -5.6334 & -0.1867 & 1.6951 \\
\hline $\mathrm{O}$ & -5.0649 & 0.8322 & -0.2069 & -4.6993 & -1.1803 & -0.7556 \\
\hline $\mathrm{O}$ & -4.1842 & 4.0426 & -1.7194 & -5.5809 & 2.2586 & -1.6074 \\
\hline $\mathrm{O}$ & -6.1354 & 2.0878 & -3.0503 & -6.5160 & -0.0668 & -3.3441 \\
\hline $\mathrm{H}$ & -3.6600 & 5.7528 & -0.8081 & -5.7654 & 3.8369 & -0.3784 \\
\hline $\mathrm{H}$ & -5.4436 & 4.7747 & 0.5213 & -6.6840 & 1.9942 & 0.8808 \\
\hline $\mathrm{H}$ & -3.5326 & 2.4593 & 1.0663 & -3.9531 & 0.6572 & 0.8038 \\
\hline $\mathrm{H}$ & -6.0986 & 2.5871 & -0.5706 & -6.4631 & -0.0926 & -0.7565 \\
\hline $\mathrm{H}$ & -3.1864 & 2.2505 & -1.4463 & -3.8342 & 1.1424 & -1.7152 \\
\hline $\mathrm{H}$ & -4.4803 & 0.9564 & -2.9335 & -4.5258 & -0.3438 & -3.3968 \\
\hline $\mathrm{H}$ & -4.2361 & 2.5286 & -3.7517 & -5.0518 & 1.2850 & -3.9129 \\
\hline $\mathrm{H}$ & -4.1128 & 6.0462 & 3.4755 & -5.2476 & 3.0858 & 3.9214 \\
\hline $\mathrm{H}$ & -5.3866 & 6.2678 & 2.2507 & -6.6565 & 2.2613 & 3.1928 \\
\hline $\mathrm{H}$ & -5.2886 & 4.7353 & 3.1684 & -5.1505 & 1.3540 & 3.4911 \\
\hline $\mathrm{H}$ & -7.6785 & 3.2735 & -3.6094 & -8.4546 & 0.3848 & -3.7140 \\
\hline $\mathrm{H}$ & -6.5707 & 4.0377 & -2.4372 & -7.7420 & 1.3123 & -2.3654 \\
\hline $\mathrm{H}$ & -6.0765 & 3.8588 & -4.1356 & -7.3155 & 1.7190 & -4.0429 \\
\hline $\mathrm{H}$ & -5.7167 & 1.6923 & 1.8800 & -5.5862 & -1.1127 & 1.3946 \\
\hline C & -6.1798 & 0.0986 & -0.6563 & -5.3190 & -2.2427 & -1.4436 \\
\hline C & -6.7059 & -0.7063 & 0.5339 & -5.5152 & -3.3783 & -0.4378 \\
\hline C & -5.6853 & -1.7576 & 0.9419 & -4.1511 & -3.8592 & 0.0286 \\
\hline C & -5.2405 & -2.5677 & -0.2747 & -3.2490 & -4.1792 & -1.1598 \\
\hline C & -4.7790 & -1.6541 & -1.4085 & -3.1909 & -3.0110 & -2.1419 \\
\hline C & -4.4553 & -2.4135 & -2.6815 & -2.4512 & -3.3654 & -3.4172 \\
\hline $\mathrm{C}$ & -7.9781 & -0.1426 & 2.4793 & -7.0910 & -3.7648 & 1.3174 \\
\hline C & -2.4749 & -1.4551 & -3.5237 & -1.0984 & -1.4863 & -3.8445 \\
\hline $\mathrm{O}$ & -6.9581 & 0.2292 & 1.5626 & -6.2774 & -2.8305 & 0.6205 \\
\hline $\mathrm{O}$ & -6.2497 & -2.6017 & 1.9156 & -4.2903 & -4.9805 & 0.8671 \\
\hline $\mathrm{O}$ & -4.1710 & -3.3911 & 0.1695 & -1.9584 & -4.4261 & -0.6169 \\
\hline $\mathrm{O}$ & -5.8304 & -0.7436 & -1.7198 & -4.5211 & -2.6547 & -2.5183 \\
\hline $\mathrm{O}$ & -3.8775 & -1.5821 & -3.6655 & -2.2258 & -2.2427 & -4.2430 \\
\hline $\mathrm{H}$ & -6.9490 & 0.7715 & -1.0431 & -6.2722 & -1.9208 & -1.8697 \\
\hline $\mathrm{H}$ & -7.6394 & -1.2054 & 0.2354 & -6.0628 & -4.1995 & -0.9219 \\
\hline
\end{tabular}




\begin{tabular}{|c|c|c|c|c|c|c|}
\hline $\mathrm{H}$ & -4.7957 & -1.2339 & 1.3293 & -3.6830 & -3.0198 & 0.5680 \\
\hline $\mathrm{H}$ & -6.0810 & -3.1865 & -0.6221 & -3.6265 & -5.0744 & -1.6760 \\
\hline $\mathrm{H}$ & -3.8971 & -1.0926 & -1.0712 & -2.7059 & -2.1606 & -1.6441 \\
\hline $\mathrm{H}$ & -5.3897 & -2.8074 & -3.0975 & -3.0686 & -4.0662 & -3.9907 \\
\hline $\mathrm{H}$ & -3.7935 & -3.2578 & -2.4456 & -1.5039 & -3.8591 & -3.1643 \\
\hline $\mathrm{H}$ & -8.1029 & 0.6976 & 3.1634 & -7.6098 & -3.2048 & 2.0970 \\
\hline $\mathrm{H}$ & -8.9224 & -0.3189 & 1.9505 & -7.8289 & -4.2088 & 0.6390 \\
\hline $\mathrm{H}$ & -7.6993 & -1.0407 & 3.0344 & -6.4834 & -4.5539 & 1.7672 \\
\hline $\mathrm{H}$ & -2.1224 & -0.8476 & -4.3596 & -0.9379 & -0.7250 & -4.6109 \\
\hline $\mathrm{H}$ & -2.1979 & -0.9524 & -2.5854 & -1.2563 & -0.9836 & -2.8795 \\
\hline $\mathrm{H}$ & -1.9900 & -2.4387 & -3.5481 & -0.2094 & -2.1257 & -3.7744 \\
\hline $\mathrm{H}$ & -5.5914 & -3.2872 & 2.1342 & -3.3990 & -5.2379 & 1.1697 \\
\hline C & -4.2887 & -4.7829 & 0.0582 & -1.4135 & -5.7055 & -0.7728 \\
\hline C & -3.7953 & -5.3924 & 1.3730 & -0.7157 & -6.0783 & 0.5372 \\
\hline C & -2.3200 & -5.0748 & 1.5445 & 0.4806 & -5.1657 & 0.7615 \\
\hline $\mathrm{C}$ & -1.5409 & -5.5089 & 0.3066 & 1.3879 & -5.2392 & -0.4653 \\
\hline C & -2.1505 & -4.9483 & -0.9784 & 0.6016 & -4.8673 & -1.7245 \\
\hline $\mathrm{C}$ & -1.5107 & -5.5243 & -2.2284 & 1.4154 & -4.9793 & -3.0003 \\
\hline C & -4.8004 & -5.7138 & 3.5136 & -1.4801 & -6.8831 & 2.6521 \\
\hline C & -2.9811 & -5.2999 & -4.0841 & -0.0097 & -5.2944 & -4.8779 \\
\hline $\mathrm{O}$ & -4.5751 & -4.8409 & 2.4137 & -1.6782 & -5.9863 & 1.5659 \\
\hline $\mathrm{O}$ & -1.8213 & -5.7236 & 2.6875 & 1.1636 & -5.5694 & 1.9222 \\
\hline $\mathrm{O}$ & -0.2146 & -5.0295 & 0.4884 & 2.4556 & -4.3373 & -0.2265 \\
\hline $\mathrm{O}$ & -3.5414 & -5.2740 & -1.0247 & -0.5126 & -5.7547 & -1.8496 \\
\hline $\mathrm{O}$ & -1.8592 & -4.7913 & -3.3837 & 0.7492 & -4.3879 & -4.0974 \\
\hline $\mathrm{H}$ & -5.3277 & -5.0715 & -0.1298 & -2.1971 & -6.4360 & -0.9990 \\
\hline $\mathrm{H}$ & -3.9381 & -6.4812 & 1.3243 & -0.3591 & -7.1150 & 0.4492 \\
\hline $\mathrm{H}$ & -2.2311 & -3.9808 & 1.6350 & 0.1161 & -4.1285 & 0.8568 \\
\hline $\mathrm{H}$ & -1.5457 & -6.6080 & 0.2598 & 1.7725 & -6.2656 & -0.5613 \\
\hline $\mathrm{H}$ & -2.0186 & -3.8566 & -0.9912 & 0.2442 & -3.8313 & -1.6249 \\
\hline $\mathrm{H}$ & -1.7976 & -6.5811 & -2.3282 & 1.6401 & -6.0377 & -3.1978 \\
\hline $\mathrm{H}$ & -0.4243 & -5.4751 & -2.1145 & 2.3604 & -4.4475 & -2.8595 \\
\hline $\mathrm{H}$ & -5.4207 & -5.1670 & 4.2242 & -2.3740 & -6.8239 & 3.2748 \\
\hline $\mathrm{H}$ & -5.3335 & -6.6143 & 3.1870 & -1.3674 & -7.9107 & 2.2871 \\
\hline $\mathrm{H}$ & -3.8553 & -5.9961 & 3.9822 & -0.5978 & -6.6023 & 3.2318 \\
\hline $\mathrm{H}$ & -3.1968 & -4.5930 & -4.8883 & -0.5169 & -4.7025 & -5.6427 \\
\hline $\mathrm{H}$ & -2.7565 & -6.2825 & -4.5182 & 0.6432 & -6.0300 & -5.3651 \\
\hline $\mathrm{H}$ & -3.8578 & -5.3866 & -3.4349 & -0.7557 & -5.8208 & -4.2742 \\
\hline $\mathrm{H}$ & -0.8637 & -5.5415 & 2.7345 & 2.0453 & -5.1509 & 1.9130 \\
\hline C & 0.8236 & -5.9699 & 0.4723 & 3.7693 & -4.7710 & -0.4737 \\
\hline C & 1.8026 & -5.6413 & 1.6089 & 4.6109 & -4.4475 & 0.7654 \\
\hline C & 2.6361 & -4.4035 & 1.3054 & 4.7436 & -2.9407 & 0.9253 \\
\hline C & 3.1956 & -4.4831 & -0.1085 & 5.2040 & -2.3151 & -0.3850 \\
\hline C & 2.0810 & -4.7389 & -1.1160 & 4.3081 & -2.7250 & -1.5487 \\
\hline C & 2.5774 & -4.7922 & -2.5530 & 4.7810 & -2.1725 & -2.8862 \\
\hline C & 1.7042 & -5.8515 & 3.9883 & 4.7662 & -5.3256 & 2.9884 \\
\hline C & 3.6034 & -6.9352 & -2.6247 & 6.7003 & -3.5097 & -3.2903 \\
\hline $\mathrm{O}$ & 1.0338 & -5.4911 & 2.7861 & 3.9530 & -5.0629 & 1.8530 \\
\hline $\mathrm{O}$ & 3.6888 & -4.3185 & 2.2404 & 5.6718 & -2.6499 & 1.9459 \\
\hline $\mathrm{O}$ & 3.8420 & -3.2452 & -0.3333 & 5.1724 & -0.9142 & -0.1689 \\
\hline $\mathrm{O}$ & 1.4712 & -5.9895 & -0.7748 & 4.2978 & -4.1564 & -1.6191 \\
\hline $\mathrm{O}$ & 3.7655 & -5.5319 & -2.7282 & 6.1807 & -2.2128 & -3.0623 \\
\hline $\mathrm{H}$ & 0.4256 & -6.9790 & 0.6195 & 3.7878 & -5.8482 & -0.6682 \\
\hline $\mathrm{H}$ & 2.4879 & -6.4960 & 1.7099 & 5.6106 & -4.8871 & 0.6295 \\
\hline $\mathrm{H}$ & 1.9812 & -3.5169 & 1.3658 & 3.7500 & -2.5351 & 1.1689 \\
\hline $\mathrm{H}$ & 3.9215 & -5.3068 & -0.1591 & 6.2329 & -2.6405 & -0.5912 \\
\hline
\end{tabular}




\begin{tabular}{|c|c|c|c|c|c|c|}
\hline $\mathrm{H}$ & 1.3315 & -3.9361 & -1.0464 & 3.2855 & -2.3632 & -1.3672 \\
\hline $\mathrm{H}$ & 2.8089 & -3.7713 & -2.8716 & 4.5002 & -1.1178 & -2.9505 \\
\hline $\mathrm{H}$ & 1.7728 & -5.1905 & -3.1900 & 4.2708 & -2.7263 & -3.6895 \\
\hline $\mathrm{H}$ & 0.9743 & -5.7521 & 4.7924 & 4.1528 & -5.9032 & 3.6811 \\
\hline $\mathrm{H}$ & 2.0498 & -6.8907 & 3.9397 & 5.6430 & -5.9186 & 2.7021 \\
\hline $\mathrm{H}$ & 2.5558 & -5.1931 & 4.1725 & 5.0964 & -4.3982 & 3.4607 \\
\hline $\mathrm{H}$ & 4.5211 & -7.3905 & -3.0006 & 7.7363 & -3.3873 & -3.6107 \\
\hline $\mathrm{H}$ & 3.4355 & -7.2528 & -1.5905 & 6.6698 & -4.1294 & -2.3874 \\
\hline $\mathrm{H}$ & 2.7554 & -7.2759 & -3.2330 & 6.1361 & -4.0241 & -4.0793 \\
\hline $\mathrm{H}$ & 4.3785 & -3.7476 & 1.8525 & 5.9260 & -1.7140 & 1.8474 \\
\hline C & 5.0446 & -3.2446 & -1.0655 & 6.2230 & -0.1409 & -0.6853 \\
\hline C & 6.1138 & -2.5867 & -0.1894 & 6.6953 & 0.7886 & 0.4385 \\
\hline C & 5.7627 & -1.1247 & 0.0284 & 5.6191 & 1.8156 & 0.7545 \\
\hline C & 5.4217 & -0.4203 & -1.2818 & 5.0896 & 2.4805 & -0.5121 \\
\hline C & 4.4034 & -1.2147 & -2.0934 & 4.6881 & 1.4402 & -1.5510 \\
\hline $\mathrm{C}$ & 4.1789 & -0.6441 & -3.4807 & 4.2889 & 2.0547 & -2.8802 \\
\hline C & 7.3452 & -3.2700 & 1.7475 & 7.9853 & 0.4516 & 2.4228 \\
\hline $\mathrm{C}$ & 1.8415 & -0.5474 & -3.7068 & 2.2259 & 1.0533 & -3.4019 \\
\hline $\mathrm{O}$ & 6.1250 & -3.3203 & 1.0212 & 6.9738 & -0.0383 & 1.5526 \\
\hline $\mathrm{O}$ & 6.8306 & -0.4653 & 0.6706 & 6.1274 & 2.7809 & 1.6440 \\
\hline $\mathrm{O}$ & 4.8608 & 0.8316 & -0.9065 & 3.9525 & 3.2373 & -0.1172 \\
\hline $\mathrm{O}$ & 4.8839 & -2.5466 & -2.2694 & 5.8089 & 0.5961 & -1.8048 \\
\hline $\mathrm{O}$ & 3.0337 & -1.1919 & -4.0991 & 3.6020 & 1.1375 & -3.7055 \\
\hline $\mathrm{H}$ & 5.3289 & -4.2639 & -1.3371 & 7.0383 & -0.7819 & -1.0313 \\
\hline $\mathrm{H}$ & 7.0875 & -2.6686 & -0.6942 & 7.6082 & 1.3037 & 0.1064 \\
\hline $\mathrm{H}$ & 4.8555 & -1.0965 & 0.6486 & 4.7751 & 1.2704 & 1.2000 \\
\hline $\mathrm{H}$ & 6.3378 & -0.2724 & -1.8724 & 5.8637 & 3.1386 & -0.9327 \\
\hline $\mathrm{H}$ & 3.4567 & -1.2329 & -1.5372 & 3.8573 & 0.8432 & -1.1485 \\
\hline $\mathrm{H}$ & 5.0396 & -0.9046 & -4.1052 & 5.1999 & 2.3492 & -3.4110 \\
\hline $\mathrm{H}$ & 4.1111 & 0.4520 & -3.4222 & 3.6842 & 2.9551 & -2.7038 \\
\hline $\mathrm{H}$ & 7.2289 & -3.9451 & 2.5966 & 8.1210 & -0.3026 & 3.1988 \\
\hline $\mathrm{H}$ & 8.1773 & -3.6166 & 1.1232 & 8.9286 & 0.5825 & 1.8793 \\
\hline $\mathrm{H}$ & 7.5535 & -2.2570 & 2.0992 & 7.6888 & 1.4017 & 2.8718 \\
\hline $\mathrm{H}$ & 1.0216 & -1.0152 & -4.2554 & 1.7830 & 0.3336 & -4.0927 \\
\hline $\mathrm{H}$ & 1.6442 & -0.6457 & -2.6305 & 2.0440 & 0.7121 & -2.3734 \\
\hline $\mathrm{H}$ & 1.8781 & 0.5235 & -3.9556 & 1.7369 & 2.0292 & -3.5352 \\
\hline $\mathrm{H}$ & 6.5495 & 0.4570 & 0.8197 & 5.3943 & 3.3841 & 1.8687 \\
\hline $\mathrm{H}$ & 3.2791 & 2.8222 & -4.3175 & 2.1086 & 4.8157 & -3.5943 \\
\hline C & 3.8001 & 1.2515 & 3.0651 & 1.7756 & -1.9802 & 3.7419 \\
\hline $\mathrm{C}$ & -0.9510 & 1.1424 & 0.6784 & -1.9916 & 1.5342 & 4.4967 \\
\hline C & 3.0049 & -1.4028 & 3.3480 & 2.4063 & 0.1393 & 2.0561 \\
\hline C & 4.7440 & 0.2266 & 3.0165 & 3.0988 & -1.5632 & 3.6072 \\
\hline C & -0.8790 & -1.3985 & 1.7529 & -0.8001 & 2.6622 & 2.2811 \\
\hline C & -1.6632 & 0.1175 & 0.0653 & -1.9966 & 2.9176 & 4.3504 \\
\hline C & 4.3533 & -1.1011 & 3.1559 & 3.4185 & -0.4946 & 2.7763 \\
\hline C & -1.6212 & -1.1684 & 0.5993 & -1.3958 & 3.4874 & 3.2325 \\
\hline C & -1.2919 & 5.0283 & 4.2017 & -4.5932 & -2.9753 & 3.6569 \\
\hline C & 1.9807 & 3.2505 & 3.9269 & -0.8490 & -3.0799 & 3.6018 \\
\hline C & -0.6965 & 3.0026 & 3.0880 & -2.9683 & -1.2264 & 3.4658 \\
\hline C & 0.8633 & 4.0548 & 4.5636 & -2.2773 & -3.4908 & 3.2882 \\
\hline C & 0.5976 & -0.6773 & 3.6509 & -0.0370 & 0.4145 & 1.4308 \\
\hline C & 2.4504 & 0.9544 & 3.2844 & 0.7533 & -1.3301 & 3.0413 \\
\hline C & -0.2119 & 0.9222 & 1.8469 & -1.3990 & 0.6960 & 3.5463 \\
\hline C & 2.0562 & -0.3891 & 3.4275 & 1.0826 & -0.2766 & 2.1662 \\
\hline C & -0.1938 & -0.3667 & 2.4008 & -0.7965 & 1.2722 & 2.4184 \\
\hline $\mathrm{C}$ & 0.4278 & 2.1593 & 2.4627 & -1.5364 & -0.7965 & 3.8082 \\
\hline
\end{tabular}




\begin{tabular}{|c|c|c|c|c|c|c|c|c|c|}
\hline $\mathrm{N}$ & -0.2076 & 4.2692 & 3.6047 & -3.2191 & -2.5811 & 3.9230 & & & \\
\hline $\mathrm{N}$ & 1.4658 & 1.9562 & 3.4861 & -0.6090 & -1.7191 & 3.1201 & & & \\
\hline $\mathrm{H}$ & 4.1170 & 2.2811 & 2.9206 & 1.5416 & -2.8082 & 4.4022 & & & \\
\hline $\mathrm{H}$ & -0.9974 & 2.1474 & 0.2589 & -2.4554 & 1.0875 & 5.3736 & & & \\
\hline $\mathrm{H}$ & 2.6948 & -2.4396 & 3.4519 & 2.6391 & 0.9685 & 1.3894 & & & \\
\hline $\mathrm{H}$ & 5.7871 & 0.4681 & 2.8449 & 3.8852 & -2.0825 & 4.1454 & & & \\
\hline $\mathrm{H}$ & -0.8227 & -2.4004 & 2.1720 & -0.3326 & 3.1060 & 1.4041 & & & \\
\hline $\mathrm{H}$ & -2.2545 & 0.3296 & -0.8212 & -2.4594 & 3.5425 & 5.1072 & & & \\
\hline $\mathrm{H}$ & 5.0898 & -1.8979 & 3.0980 & 4.4534 & -0.1827 & 2.6666 & & & \\
\hline $\mathrm{H}$ & -2.1763 & -1.9813 & 0.1383 & -1.3891 & 4.5654 & 3.0936 & & & \\
\hline $\mathrm{H}$ & -0.9355 & 6.0229 & 4.4869 & -4.7708 & -3.9790 & 4.0557 & & & \\
\hline $\mathrm{H}$ & -2.0953 & 5.1394 & 3.4722 & -5.2777 & -2.2771 & 4.1488 & & & \\
\hline $\mathrm{H}$ & -1.7033 & 4.5330 & 5.0994 & -4.8263 & -2.9916 & 2.5805 & & & \\
\hline $\mathrm{H}$ & 2.7699 & 3.0763 & 4.6622 & -0.1562 & -3.7666 & 3.1049 & & & \\
\hline $\mathrm{H}$ & 2.4122 & 3.8230 & 3.0882 & -0.6880 & -3.1544 & 4.6916 & & & \\
\hline $\mathrm{H}$ & -1.4576 & 3.2172 & 2.3265 & -3.6780 & -0.5515 & 3.9546 & & & \\
\hline $\mathrm{H}$ & -1.1730 & 2.3893 & 3.8792 & -3.1125 & -1.1198 & 2.3704 & & & \\
\hline $\mathrm{H}$ & 0.4983 & 3.5182 & 5.4601 & -2.4003 & -3.5100 & 2.1907 & & & \\
\hline $\mathrm{H}$ & 1.2474 & 5.0297 & 4.8846 & -2.4573 & -4.5045 & 3.6602 & & & \\
\hline $\mathrm{H}$ & 0.4568 & -1.7272 & 3.9207 & 0.3616 & 1.0395 & 0.6259 & & & \\
\hline $\mathrm{H}$ & 0.2344 & -0.0564 & 4.4787 & -0.7009 & -0.3394 & 0.9914 & & & \\
\hline \multirow[t]{2}{*}{$\mathrm{H}$} & 0.8680 & 2.7451 & 1.6311 & -1.4161 & -0.9382 & 4.8980 & & & \\
\hline & \multicolumn{3}{|c|}{ CR2 } & \multicolumn{3}{|c|}{ NR2 } & \multicolumn{3}{|c|}{ M2 } \\
\hline atom & $\mathbf{x}$ & $\mathbf{y}$ & $\mathbf{Z}$ & $\mathbf{x}$ & $\mathbf{y}$ & $\mathbf{Z}$ & $\mathbf{x}$ & $\mathbf{y}$ & $\mathbf{Z}$ \\
\hline $\mathrm{C}$ & -3.0346 & -3.8483 & -2.5247 & -4.6905 & 1.4812 & -2.5190 & -0.7501 & 5.9341 & 1.1733 \\
\hline $\mathrm{C}$ & -3.3485 & -3.3712 & -3.9454 & -4.3799 & 2.4021 & -3.6999 & -1.8850 & 6.0556 & 2.1941 \\
\hline $\mathrm{C}$ & -3.3705 & -1.8517 & -3.9953 & -3.1058 & 3.1890 & -3.4242 & -2.9324 & 4.9837 & 1.9345 \\
\hline $\mathrm{C}$ & -4.2922 & -1.3131 & -2.9053 & -3.1859 & 3.8551 & -2.0535 & -3.3544 & 5.0292 & 0.4711 \\
\hline $\mathrm{C}$ & -3.9234 & -1.8820 & -1.5414 & -3.5662 & 2.8563 & -0.9687 & -2.1559 & 4.9577 & -0.4696 \\
\hline $\mathrm{C}$ & -4.8528 & -1.4359 & -0.4266 & -3.7008 & 3.4714 & 0.4169 & -2.5428 & 5.0929 & -1.9365 \\
\hline $\mathrm{C}$ & -2.8165 & -4.2103 & -6.1135 & -4.6394 & 2.1973 & -6.0593 & -1.9665 & 6.6814 & 4.4932 \\
\hline $\mathrm{C}$ & -6.7002 & -2.8875 & -0.8119 & -5.7461 & 4.6368 & 0.1885 & -3.0898 & 7.4005 & -2.0472 \\
\hline $\mathrm{O}$ & -2.3619 & -3.9249 & -4.7954 & -4.2760 & 1.5675 & -4.8371 & -1.3013 & 5.9390 & 3.4778 \\
\hline $\mathrm{O}$ & -3.8064 & -1.4216 & -5.2626 & -2.9097 & 4.1654 & -4.4212 & -4.0506 & 5.1930 & 2.7658 \\
\hline $\mathrm{O}$ & -4.1075 & 0.0929 & -2.9091 & -1.8798 & 4.3570 & -1.8188 & -4.2066 & 3.9119 & 0.2922 \\
\hline $\mathrm{O}$ & -3.9363 & -3.3106 & -1.5933 & -4.7864 & 2.1957 & -1.3140 & -1.2341 & 6.0032 & -0.1431 \\
\hline $\mathrm{O}$ & -6.2250 & -1.5568 & -0.7222 & -4.3542 & 4.7202 & 0.4353 & -3.5375 & 6.0646 & -2.1812 \\
\hline $\mathrm{H}$ & -3.1510 & -4.9353 & -2.4699 & -5.6634 & 1.0026 & -2.6699 & -0.0569 & 6.7735 & 1.2894 \\
\hline $\mathrm{H}$ & -4.3435 & -3.7542 & -4.2171 & -5.2197 & 3.1038 & -3.8142 & -2.3464 & 7.0469 & 2.0738 \\
\hline $\mathrm{H}$ & -2.3513 & -1.4919 & -3.7809 & -2.2685 & 2.4732 & -3.3988 & -2.4703 & 4.0029 & 2.1284 \\
\hline $\mathrm{H}$ & -5.3337 & -1.5650 & -3.1480 & -3.9117 & 4.6788 & -2.0954 & -3.9088 & 5.9610 & 0.2939 \\
\hline $\mathrm{H}$ & -2.9089 & -1.5365 & -1.2995 & -2.7637 & 2.1157 & -0.9310 & -1.6670 & 3.9820 & -0.3282 \\
\hline $\mathrm{H}$ & -4.6914 & -0.3733 & -0.2261 & -2.6982 & 3.6431 & 0.8193 & -2.9572 & 4.1431 & -2.2825 \\
\hline $\mathrm{H}$ & -1.9735 & -4.6517 & -6.6458 & -4.5476 & 1.4419 & -6.8402 & -1.4079 & 6.5198 & 5.4155 \\
\hline $\mathrm{H}$ & -3.6446 & -4.9286 & -6.0890 & -5.6769 & 2.5502 & -6.0186 & -1.9654 & 7.7511 & 4.2524 \\
\hline $\mathrm{H}$ & -3.1397 & -3.2965 & -6.6158 & -3.9776 & 3.0392 & -6.2742 & -2.9952 & 6.3352 & 4.6132 \\
\hline $\mathrm{H}$ & -7.7865 & -2.8470 & -0.7102 & -6.1698 & 5.6184 & 0.4057 & -3.8753 & 8.0432 & -2.4484 \\
\hline $\mathrm{H}$ & -6.4403 & -3.3519 & -1.7691 & -5.9621 & 4.3687 & -0.8517 & -2.9007 & 7.6680 & -1.0016 \\
\hline $\mathrm{H}$ & -6.2835 & -3.5123 & -0.0107 & -6.2132 & 3.8868 & 0.8412 & -2.1644 & 7.5618 & -2.6155 \\
\hline $\mathrm{H}$ & -3.9385 & -0.4565 & -5.2130 & -2.2297 & 4.7808 & -4.0905 & -4.7819 & 4.6563 & 2.4084 \\
\hline $\mathrm{C}$ & -5.2281 & 0.9332 & -2.9432 & -1.7408 & 5.5909 & -1.1669 & -5.3476 & 4.0623 & -0.5099 \\
\hline $\mathrm{C}$ & -4.9277 & 2.0267 & -3.9721 & -0.7911 & 6.4429 & -2.0165 & -6.5281 & 3.4879 & 0.2774 \\
\hline $\mathrm{C}$ & -3.7587 & 2.8721 & -3.4856 & 0.6119 & 5.8521 & -1.9785 & -6.3868 & 1.9768 & 0.4157 \\
\hline $\mathrm{C}$ & -3.9976 & 3.3456 & -2.0543 & 1.0476 & 5.5843 & -0.5384 & -6.0861 & 1.3347 & -0.9395 \\
\hline $\mathrm{C}$ & -4.3988 & 2.2046 & -1.1196 & 0.0106 & 4.7403 & 0.1943 & -4.8918 & 2.0149 & -1.6047 \\
\hline $\mathrm{C}$ & -4.8715 & 2.7028 & 0.2374 & 0.3177 & 4.5106 & 1.6685 & -4.5869 & 1.4943 & -2.9892 \\
\hline
\end{tabular}




\begin{tabular}{|c|c|c|c|c|c|c|c|c|c|}
\hline $\mathrm{C}$ & -4.9324 & 2.1233 & -6.3593 & -1.0368 & 7.6173 & -4.0804 & -7.7993 & 4.2512 & 2.1516 \\
\hline $\mathrm{C}$ & -4.0572 & 1.2258 & 1.8881 & 0.6915 & 2.2102 & 1.9870 & -3.0070 & 1.6533 & -4.7057 \\
\hline $\mathrm{O}$ & -4.6455 & 1.3678 & -5.1902 & -1.3364 & 6.4545 & -3.3209 & -6.5272 & 4.1481 & 1.5275 \\
\hline $\mathrm{O}$ & -3.5847 & 3.9836 & -4.3340 & 1.5170 & 6.7305 & -2.6063 & -7.5745 & 1.4487 & 0.9549 \\
\hline $\mathrm{O}$ & -2.7682 & 3.9339 & -1.6589 & 2.2766 & 4.8756 & -0.6123 & -5.8296 & -0.0369 & -0.6726 \\
\hline $\mathrm{O}$ & -5.4971 & 1.4928 & -1.6864 & -1.2451 & 5.4176 & 0.1345 & -5.1937 & 3.4030 & -1.7378 \\
\hline $\mathrm{O}$ & -5.1613 & 1.6305 & 1.1064 & -0.2223 & 3.2808 & 2.1204 & -3.3613 & 2.0550 & -3.4018 \\
\hline $\mathrm{H}$ & -6.1247 & 0.3729 & -3.2273 & -2.7126 & 6.0796 & -1.0560 & -5.5124 & 5.1161 & -0.7505 \\
\hline $\mathrm{H}$ & -5.8192 & 2.6615 & -4.0794 & -0.7673 & 7.4617 & -1.6027 & -7.4556 & 3.7100 & -0.2705 \\
\hline $\mathrm{H}$ & -2.8633 & 2.2315 & -3.4800 & 0.5780 & 4.8806 & -2.4962 & -5.5252 & 1.7782 & 1.0734 \\
\hline $\mathrm{H}$ & -4.7940 & 4.1054 & -2.0724 & 1.1832 & 6.5394 & -0.0106 & -6.9696 & 1.4388 & -1.5863 \\
\hline $\mathrm{H}$ & -3.5371 & 1.5303 & -0.9983 & -0.0652 & 3.7656 & -0.3102 & -3.9997 & 1.8834 & -0.9770 \\
\hline $\mathrm{H}$ & -5.8001 & 3.2666 & 0.0905 & -0.1516 & 5.3123 & 2.2460 & -5.3982 & 1.7844 & -3.6763 \\
\hline $\mathrm{H}$ & -4.1159 & 3.3692 & 0.6732 & 1.4013 & 4.5501 & 1.8416 & -4.5326 & 0.3966 & -2.9691 \\
\hline $\mathrm{H}$ & -4.7325 & 1.4705 & -7.2097 & -1.5604 & 7.5128 & -5.0314 & -7.6558 & 4.8435 & 3.0561 \\
\hline $\mathrm{H}$ & -5.9876 & 2.4212 & -6.3747 & -1.4002 & 8.5159 & -3.5674 & -8.5095 & 4.7666 & 1.4937 \\
\hline $\mathrm{H}$ & -4.3029 & 3.0139 & -6.4149 & 0.0379 & 7.7081 & -4.2528 & -8.1938 & 3.2656 & 2.4078 \\
\hline $\mathrm{H}$ & -4.3149 & 0.2617 & 2.3316 & 0.1433 & 1.2798 & 2.1534 & -2.0747 & 2.1583 & -4.9617 \\
\hline $\mathrm{H}$ & -3.1455 & 1.1090 & 1.2833 & 1.1289 & 2.1731 & 0.9794 & -2.8564 & 0.5661 & -4.7600 \\
\hline $\mathrm{H}$ & -3.8421 & 1.9451 & 2.6935 & 1.5031 & 2.2897 & 2.7230 & -3.7800 & 1.9350 & -5.4330 \\
\hline $\mathrm{H}$ & -2.9936 & 4.6098 & -3.8771 & 2.3859 & 6.2883 & -2.6311 & -7.5142 & 0.4763 & 0.9127 \\
\hline $\mathrm{C}$ & -2.7983 & 5.0939 & -0.8731 & 3.4365 & 5.5294 & -0.1771 & -6.5185 & -1.0074 & -1.4145 \\
\hline $\mathrm{C}$ & -1.9255 & 6.1471 & -1.5639 & 4.5679 & 5.1687 & -1.1437 & -7.0516 & -2.0554 & -0.4315 \\
\hline $\mathrm{C}$ & -0.4523 & 5.7611 & -1.4936 & 4.8748 & 3.6801 & -1.0598 & -5.9009 & -2.8284 & 0.1956 \\
\hline $\mathrm{C}$ & -0.0873 & 5.3915 & -0.0588 & 5.0840 & 3.2695 & 0.3918 & -4.9971 & -3.3618 & -0.9078 \\
\hline $\mathrm{C}$ & -1.0144 & 4.3042 & 0.4711 & 3.9509 & 3.7501 & 1.2930 & -4.5332 & -2.2457 & -1.8392 \\
\hline $\mathrm{C}$ & -0.6954 & 3.8726 & 1.8909 & 4.1902 & 3.4460 & 2.7653 & -3.6761 & -2.7637 & -2.9865 \\
\hline $\mathrm{C}$ & -2.1989 & 7.4999 & -3.5150 & 5.1974 & 5.9552 & -3.3057 & -8.9218 & -2.0971 & 1.0521 \\
\hline $\mathrm{C}$ & -1.6750 & 5.5656 & 3.2328 & 5.7922 & 5.1155 & 3.3113 & -5.3115 & -3.8088 & -4.3556 \\
\hline $\mathrm{O}$ & -2.4062 & 6.2409 & -2.8891 & 4.1444 & 5.5537 & -2.4373 & -7.8214 & -1.3642 & 0.5301 \\
\hline $\mathrm{O}$ & 0.3286 & 6.8435 & -1.9406 & 6.0279 & 3.3859 & -1.8159 & -6.4160 & -3.8885 & 0.9658 \\
\hline $\mathrm{O}$ & 1.2655 & 4.9658 & -0.0786 & 5.1318 & 1.8516 & 0.3719 & -3.9010 & -3.9814 & -0.2589 \\
\hline $\mathrm{O}$ & -2.3478 & 4.8249 & 0.4284 & 3.7628 & 5.1586 & 1.1389 & -5.6900 & -1.5984 & -2.3830 \\
\hline $\mathrm{O}$ & -0.4855 & 4.9366 & 2.7909 & 5.4940 & 3.7334 & 3.2196 & -4.1497 & -3.9617 & -3.5610 \\
\hline $\mathrm{H}$ & -3.8235 & 5.4662 & -0.7730 & 3.2895 & 6.6142 & -0.1635 & -7.3502 & -0.5535 & -1.9632 \\
\hline $\mathrm{H}$ & -2.0599 & 7.1060 & -1.0415 & 5.4610 & 5.7387 & -0.8486 & -7.6863 & -2.7571 & -0.9925 \\
\hline $\mathrm{H}$ & -0.3007 & 4.8704 & -2.1252 & 3.9984 & 3.1341 & -1.4427 & -5.3161 & -2.1310 & 0.8166 \\
\hline $\mathrm{H}$ & -0.1943 & 6.2810 & 0.5772 & 6.0428 & 3.6793 & 0.7386 & -5.5582 & -4.1043 & -1.4917 \\
\hline $\mathrm{H}$ & -0.9502 & 3.4142 & -0.1713 & 3.0371 & 3.2272 & 0.9834 & -3.9425 & -1.5144 & -1.2677 \\
\hline $\mathrm{H}$ & 0.2367 & 3.2980 & 1.8761 & 4.0486 & 2.3713 & 2.9161 & -2.6760 & -2.9911 & -2.6067 \\
\hline $\mathrm{H}$ & -1.5057 & 3.2162 & 2.2445 & 3.4421 & 3.9927 & 3.3602 & -3.5968 & -1.9715 & -3.7473 \\
\hline $\mathrm{H}$ & -2.6884 & 7.4476 & -4.4882 & 4.7318 & 6.2478 & -4.2472 & -9.4366 & -1.4339 & 1.7481 \\
\hline $\mathrm{H}$ & -2.6574 & 8.3019 & -2.9242 & 5.7362 & 6.8132 & -2.8868 & -9.6108 & -2.3805 & 0.2478 \\
\hline $\mathrm{H}$ & -1.1341 & 7.7072 & -3.6411 & 5.8956 & 5.1323 & -3.4733 & -8.5803 & -2.9941 & 1.5731 \\
\hline $\mathrm{H}$ & -1.4092 & 6.1853 & 4.0913 & 6.7184 & 5.2035 & 3.8812 & -5.4412 & -4.7376 & -4.9136 \\
\hline $\mathrm{H}$ & -2.1211 & 6.1919 & 2.4540 & 5.9264 & 5.5708 & 2.3245 & -6.2035 & -3.6223 & -3.7476 \\
\hline $\mathrm{H}$ & -2.4169 & 4.8176 & 3.5460 & 4.9913 & 5.6533 & 3.8344 & -5.1961 & -2.9758 & -5.0614 \\
\hline $\mathrm{H}$ & 1.2521 & 6.6723 & -1.6785 & 6.3821 & 2.5395 & -1.4876 & -5.6852 & -4.5102 & 1.1388 \\
\hline $\mathrm{C}$ & 2.1311 & 5.4999 & 0.8901 & 5.9730 & 1.2139 & 1.3015 & -3.4648 & -5.2271 & -0.7405 \\
\hline $\mathrm{C}$ & 3.3977 & 5.9762 & 0.1665 & 7.0147 & 0.4101 & 0.5143 & -3.3500 & -6.1656 & 0.4661 \\
\hline $\mathrm{C}$ & 4.1596 & 4.7787 & -0.3777 & 6.3592 & -0.7768 & -0.1757 & -2.2280 & -5.6966 & 1.3804 \\
\hline $\mathrm{C}$ & 4.3976 & 3.7709 & 0.7417 & 5.4940 & -1.5566 & 0.8125 & -0.9485 & -5.4771 & 0.5769 \\
\hline $\mathrm{C}$ & 3.0893 & 3.3812 & 1.4226 & 4.4832 & -0.6337 & 1.4864 & -1.1925 & -4.5561 & -0.6163 \\
\hline $\mathrm{C}$ & 3.3101 & 2.5072 & 2.6476 & 3.6179 & -1.3231 & 2.5367 & 0.0273 & -4.4415 & -1.5114 \\
\hline $\mathrm{C}$ & 3.8988 & 7.8971 & -1.1588 & 8.9325 & 1.0352 & -0.7674 & -4.9438 & -7.3438 & 1.8002 \\
\hline $\mathrm{C}$ & 2.1694 & 0.4830 & 2.3062 & 1.4477 & -1.3703 & 1.6676 & 0.1919 & -2.1186 & -1.8896 \\
\hline
\end{tabular}




\begin{tabular}{|c|c|c|c|c|c|c|c|c|c|}
\hline $\mathrm{O}$ & 2.9797 & 6.8567 & -0.8555 & 7.5884 & 1.3191 & -0.4049 & -4.6103 & -6.1505 & 1.1049 \\
\hline $\mathrm{O}$ & 5.3870 & 5.1981 & -0.9292 & 7.3531 & -1.6105 & -0.7265 & -2.0072 & -6.6503 & 2.3937 \\
\hline $\mathrm{O}$ & 4.9933 & 2.6254 & 0.1510 & 4.8272 & -2.5690 & 0.0709 & -0.0037 & -4.9027 & 1.4703 \\
\hline $\mathrm{O}$ & 2.4362 & 4.5631 & 1.8845 & 5.2191 & 0.3962 & 2.1546 & -2.2400 & -5.1122 & -1.4099 \\
\hline $\mathrm{O}$ & 2.1701 & 1.7339 & 2.9679 & 2.3186 & -0.7637 & 2.6053 & -0.0644 & -3.3920 & -2.4533 \\
\hline $\mathrm{H}$ & 1.6524 & 6.3369 & 1.4069 & 6.4607 & 1.9531 & 1.9433 & -4.1734 & -5.6236 & -1.4729 \\
\hline $\mathrm{H}$ & 4.0289 & 6.5095 & 0.8924 & 7.7787 & 0.0412 & 1.2143 & -3.1209 & -7.1773 & 0.1000 \\
\hline $\mathrm{H}$ & 3.5291 & 4.2939 & -1.1390 & 5.6920 & -0.3842 & -0.9594 & -2.5254 & -4.7256 & 1.8063 \\
\hline $\mathrm{H}$ & 5.0733 & 4.2107 & 1.4907 & 6.1313 & -2.0079 & 1.5874 & -0.5839 & -6.4478 & 0.2090 \\
\hline $\mathrm{H}$ & 2.4496 & 2.8581 & 0.6959 & 3.8339 & -0.1867 & 0.7206 & -1.4918 & -3.5660 & -0.2451 \\
\hline $\mathrm{H}$ & 3.5111 & 3.1669 & 3.4984 & 4.0923 & -1.1834 & 3.5133 & 0.1195 & -5.3728 & -2.0812 \\
\hline $\mathrm{H}$ & 4.1823 & 1.8601 & 2.5010 & 3.5577 & -2.3996 & 2.3330 & 0.9236 & -4.3210 & -0.8925 \\
\hline $\mathrm{H}$ & 3.4341 & 8.5088 & -1.9329 & 9.2531 & 1.8403 & -1.4296 & -5.9539 & -7.2070 & 2.1881 \\
\hline $\mathrm{H}$ & 4.0835 & 8.5168 & -0.2732 & 9.5753 & 1.0233 & 0.1210 & -4.9345 & -8.2014 & 1.1168 \\
\hline $\mathrm{H}$ & 4.8442 & 7.4885 & -1.5221 & 9.0072 & 0.0747 & -1.2818 & -4.2483 & -7.5267 & 2.6220 \\
\hline $\mathrm{H}$ & 1.2596 & -0.0458 & 2.5997 & 0.4891 & -0.8505 & 1.6981 & 0.3144 & -1.4137 & -2.7159 \\
\hline $\mathrm{H}$ & 2.1743 & 0.5986 & 1.2122 & 1.8565 & -1.3166 & 0.6460 & -0.6324 & -1.7767 & -1.2485 \\
\hline $\mathrm{H}$ & 3.0435 & -0.1087 & 2.6056 & 1.2845 & -2.4252 & 1.9276 & 1.1206 & -2.1361 & -1.3031 \\
\hline $\mathrm{H}$ & 5.7992 & 4.4216 & -1.3511 & 6.9038 & -2.2993 & -1.2509 & -1.3128 & -6.2980 & 2.9811 \\
\hline C & 6.3538 & 2.4160 & 0.4114 & 5.2898 & -3.8808 & 0.2481 & 1.1194 & -5.6701 & 1.8012 \\
\hline C & 7.0014 & 1.8738 & -0.8644 & 5.2662 & -4.5930 & -1.1076 & 1.4148 & -5.4687 & 3.2903 \\
\hline C & 6.4331 & 0.4996 & -1.1802 & 3.8314 & -4.7631 & -1.5776 & 1.8787 & -4.0432 & 3.5501 \\
\hline C & 6.5068 & -0.4151 & 0.0419 & 2.9669 & -5.3872 & -0.4838 & 3.0106 & -3.6656 & 2.5956 \\
\hline C & 5.9128 & 0.2533 & 1.2812 & 3.1204 & -4.6375 & 0.8400 & 2.6095 & -3.9420 & 1.1493 \\
\hline $\mathrm{C}$ & 6.1076 & -0.5462 & 2.5575 & 2.3972 & -5.2890 & 2.0059 & 3.7136 & -3.6885 & 0.1429 \\
\hline C & 7.7507 & 2.8831 & -2.8892 & 6.6948 & -4.5587 & -3.0172 & 0.4361 & -6.3374 & 5.2862 \\
\hline C & 5.9667 & 0.9395 & 4.4150 & 3.4150 & -4.5663 & 4.0346 & 3.3483 & -4.9052 & -1.8756 \\
\hline $\mathrm{O}$ & 6.7392 & 2.8112 & -1.8912 & 6.0317 & -3.8110 & -2.0042 & 0.2282 & -5.7794 & 3.9943 \\
\hline $\mathrm{O}$ & 7.1335 & -0.0821 & -2.2547 & 3.7894 & -5.5671 & -2.7338 & 2.3036 & -3.9224 & 4.8857 \\
\hline $\mathrm{O}$ & 5.7488 & -1.5499 & -0.3453 & 1.6532 & -5.2758 & -1.0079 & 3.2247 & -2.2785 & 2.8041 \\
\hline $\mathrm{O}$ & 6.5334 & 1.5243 & 1.4808 & 4.5060 & -4.5726 & 1.1847 & 2.2305 & -5.3133 & 1.0192 \\
\hline $\mathrm{O}$ & 5.3279 & -0.0493 & 3.6265 & 2.3272 & -4.4420 & 3.1357 & 3.1924 & -3.6845 & -1.1733 \\
\hline $\mathrm{H}$ & 6.8405 & 3.3513 & 0.7060 & 6.3104 & -3.8787 & 0.6440 & 0.9349 & -6.7307 & 1.6015 \\
\hline $\mathrm{H}$ & 8.0836 & 1.7916 & -0.6883 & 5.7266 & -5.5827 & -0.9754 & 2.2182 & -6.1646 & 3.5722 \\
\hline $\mathrm{H}$ & 5.3673 & 0.6304 & -1.4250 & 3.4269 & -3.7581 & -1.7778 & 1.0321 & -3.3706 & 3.3376 \\
\hline $\mathrm{H}$ & 7.5564 & -0.6944 & 0.2188 & 3.2449 & -6.4451 & -0.3638 & 3.9117 & -4.2393 & 2.8580 \\
\hline $\mathrm{H}$ & 4.8368 & 0.3853 & 1.1029 & 2.7251 & -3.6240 & 0.6972 & 1.7570 & -3.2933 & 0.9000 \\
\hline $\mathrm{H}$ & 7.1760 & -0.5535 & 2.8203 & 2.8947 & -6.2380 & 2.2563 & 4.4999 & -4.4488 & 0.2546 \\
\hline $\mathrm{H}$ & 5.7815 & -1.5744 & 2.3789 & 1.3675 & -5.4990 & 1.7053 & 4.1553 & -2.7045 & 0.3312 \\
\hline $\mathrm{H}$ & 7.4334 & 3.6445 & -3.6023 & 7.2645 & -3.8434 & -3.6109 & -0.5509 & -6.5641 & 5.6906 \\
\hline $\mathrm{H}$ & 8.7091 & 3.1793 & -2.4467 & 7.3820 & -5.2874 & -2.5713 & 1.0194 & -7.2632 & 5.2174 \\
\hline $\mathrm{H}$ & 7.8636 & 1.9217 & -3.3948 & 5.9716 & -5.0778 & -3.6496 & 0.9524 & -5.6274 & 5.9354 \\
\hline $\mathrm{H}$ & 5.2954 & 1.1614 & 5.2467 & 3.1896 & -3.9301 & 4.8927 & 2.8325 & -4.7834 & -2.8309 \\
\hline $\mathrm{H}$ & 6.9177 & 0.5631 & 4.8135 & 3.5194 & -5.6042 & 4.3767 & 4.4107 & -5.1128 & -2.0626 \\
\hline $\mathrm{H}$ & 6.1570 & 1.8550 & 3.8463 & 4.3581 & -4.2453 & 3.5812 & 2.9044 & -5.7410 & -1.3256 \\
\hline $\mathrm{H}$ & 6.8587 & -1.0162 & -2.3046 & 2.8554 & -5.8009 & -2.8853 & 2.7256 & -3.0483 & 4.9814 \\
\hline C & 6.1545 & -2.8312 & 0.0428 & 0.7099 & -6.2767 & -0.7553 & 4.5293 & -1.7929 & 2.9705 \\
\hline $\mathrm{C}$ & 6.0878 & -3.7185 & -1.2048 & 0.0254 & -6.6023 & -2.0890 & 4.5069 & -0.8500 & 4.1773 \\
\hline C & 4.6387 & -3.9101 & -1.6435 & -0.8451 & -5.4324 & -2.5377 & 3.6673 & 0.3865 & 3.8728 \\
\hline C & 3.7646 & -4.2942 & -0.4526 & -1.7129 & -4.9246 & -1.3881 & 4.1086 & 0.9882 & 2.5443 \\
\hline C & 3.9409 & -3.3188 & 0.7061 & -0.8614 & -4.6139 & -0.1612 & 4.1142 & -0.0520 & 1.4279 \\
\hline C & 3.0964 & -3.6523 & 1.9266 & -1.6174 & -4.0755 & 1.0427 & 4.6083 & 0.5222 & 0.1145 \\
\hline C & 7.3819 & -3.9151 & -3.2031 & 0.6886 & -7.6976 & -4.1028 & 4.4402 & -1.1839 & 6.5436 \\
\hline C & 4.2516 & -5.5230 & 2.8157 & -2.7539 & -5.9526 & 1.9275 & 6.9496 & 0.5112 & -0.2174 \\
\hline $\mathrm{O}$ & 6.8774 & -3.0682 & -2.1795 & 1.0693 & -6.8938 & -2.9948 & 3.9956 & -1.6011 & 5.2596 \\
\hline $\mathrm{O}$ & 4.5729 & -4.9150 & -2.6301 & -1.6724 & -5.8290 & -3.6097 & 3.8226 & 1.3195 & 4.9175 \\
\hline
\end{tabular}




\begin{tabular}{|c|c|c|c|c|c|c|c|c|c|}
\hline $\mathrm{O}$ & 2.4371 & -4.2980 & -0.9463 & -2.3724 & -3.7930 & -1.9259 & 3.1979 & 2.0382 & 2.2541 \\
\hline $\mathrm{O}$ & 5.3277 & -3.3310 & 1.0647 & -0.2255 & -5.8477 & 0.2021 & 4.9770 & -1.1240 & 1.8180 \\
\hline $\mathrm{O}$ & 3.0511 & -5.0229 & 2.2547 & -2.8591 & -4.6999 & 1.2775 & 5.8256 & 1.2197 & 0.2734 \\
\hline $\mathrm{H}$ & 7.1726 & -2.8146 & 0.4463 & 1.1894 & -7.1716 & -0.3441 & 5.2313 & -2.6152 & 3.1433 \\
\hline $\mathrm{H}$ & 6.5157 & -4.7018 & -0.9587 & -0.6155 & -7.4849 & -1.9449 & 5.5386 & -0.5333 & 4.3885 \\
\hline $\mathrm{H}$ & 4.2739 & -2.9456 & -2.0321 & -0.1742 & -4.6111 & -2.8379 & 2.6165 & 0.0702 & 3.7753 \\
\hline $\mathrm{H}$ & 4.0477 & -5.3027 & -0.1195 & -2.4474 & -5.6976 & -1.1208 & 5.1253 & 1.3860 & 2.6572 \\
\hline $\mathrm{H}$ & 3.6604 & -2.3075 & 0.3801 & -0.0926 & -3.8728 & -0.4210 & 3.0937 & -0.4323 & 1.2763 \\
\hline $\mathrm{H}$ & 2.0648 & -3.3596 & 1.7127 & -1.8285 & -3.0158 & 0.8790 & 3.8525 & 1.2283 & -0.2495 \\
\hline $\mathrm{H}$ & 3.4682 & -3.0584 & 2.7763 & -0.9629 & -4.1611 & 1.9233 & 4.7118 & -0.2888 & -0.6208 \\
\hline $\mathrm{H}$ & 8.0499 & -3.3044 & -3.8114 & 1.6020 & -7.9198 & -4.6555 & 4.0350 & -1.8986 & 7.2607 \\
\hline $\mathrm{H}$ & 7.9497 & -4.7466 & -2.7685 & 0.2378 & -8.6368 & -3.7596 & 5.5353 & -1.2034 & 6.5953 \\
\hline $\mathrm{H}$ & 6.5740 & -4.3152 & -3.8197 & -0.0209 & -7.1738 & -4.7473 & 4.0843 & -0.1779 & 6.7748 \\
\hline $\mathrm{H}$ & 4.0334 & -6.5205 & 3.2002 & -3.7668 & -6.2653 & 2.1869 & 7.8323 & 1.1122 & 0.0099 \\
\hline $\mathrm{H}$ & 5.0545 & -5.5855 & 2.0734 & -2.2906 & -6.7081 & 1.2840 & 7.0439 & -0.4698 & 0.2629 \\
\hline $\mathrm{H}$ & 4.5926 & -4.8837 & 3.6405 & -2.1582 & -5.8632 & 2.8466 & 6.8777 & 0.3685 & -1.3038 \\
\hline $\mathrm{H}$ & 3.6461 & -5.2125 & -2.6831 & -2.3511 & -5.1373 & -3.7134 & 3.5950 & 2.2003 & 4.5678 \\
\hline $\mathrm{C}$ & 1.5614 & -5.2864 & -0.4647 & -3.6856 & -3.5403 & -1.5109 & 3.7179 & 3.2200 & 1.6927 \\
\hline $\mathrm{C}$ & 0.9648 & -6.0051 & -1.6810 & -4.4882 & -3.1141 & -2.7520 & 3.3870 & 4.3703 & 2.6504 \\
\hline $\mathrm{C}$ & 0.0286 & -5.0745 & -2.4385 & -4.1030 & -1.7056 & -3.2015 & 1.8800 & 4.5720 & 2.6992 \\
\hline $\mathrm{C}$ & -0.9609 & -4.4244 & -1.4740 & -4.0791 & -0.7637 & -1.9981 & 1.3175 & 4.7068 & 1.2865 \\
\hline $\mathrm{C}$ & -0.2203 & -3.7285 & -0.3388 & -3.1195 & -1.3250 & -0.9592 & 1.7495 & 3.5465 & 0.3960 \\
\hline $\mathrm{C}$ & -1.1281 & -3.0859 & 0.6999 & -2.8373 & -0.4594 & 0.2650 & 1.3574 & 3.7599 & -1.0565 \\
\hline $\mathrm{C}$ & 1.8374 & -7.5966 & -3.2344 & -5.2834 & -4.2681 & -4.6811 & 4.2856 & 5.0856 & 4.7471 \\
\hline $\mathrm{C}$ & -0.8561 & -0.7746 & 0.4084 & -0.8183 & 0.5764 & -0.3973 & 0.3566 & 1.7220 & -1.7315 \\
\hline $\mathrm{O}$ & 2.0636 & -6.4239 & -2.4651 & -4.2378 & -4.0959 & -3.7355 & 3.9211 & 4.0002 & 3.9051 \\
\hline $\mathrm{O}$ & -0.6560 & -5.7970 & -3.4368 & -5.0176 & -1.2444 & -4.1718 & 1.5704 & 5.7199 & 3.4563 \\
\hline $\mathrm{O}$ & -1.7166 & -3.4794 & -2.2182 & -3.6728 & 0.5143 & -2.4573 & -0.0972 & 4.7165 & 1.4054 \\
\hline $\mathrm{O}$ & 0.5646 & -4.7142 & 0.3372 & -3.7067 & -2.5514 & -0.5167 & 3.1734 & 3.4517 & 0.4211 \\
\hline $\mathrm{O}$ & -0.5555 & -1.8933 & 1.2163 & -1.4465 & -0.3231 & 0.4932 & 1.4753 & 2.5831 & -1.8246 \\
\hline $\mathrm{H}$ & 2.0961 & -5.9910 & 0.1769 & -4.1248 & -4.4336 & -1.0578 & 4.7982 & 3.1304 & 1.5447 \\
\hline $\mathrm{H}$ & 0.3947 & -6.8765 & -1.3263 & -5.5547 & -3.1082 & -2.4790 & 3.8711 & 5.2876 & 2.2846 \\
\hline $\mathrm{H}$ & 0.6375 & -4.2699 & -2.8800 & -3.0818 & -1.7469 & -3.6128 & 1.4369 & 3.6701 & 3.1488 \\
\hline $\mathrm{H}$ & -1.6220 & -5.1929 & -1.0477 & -5.0813 & -0.7026 & -1.5492 & 1.6728 & 5.6497 & 0.8446 \\
\hline $\mathrm{H}$ & 0.4415 & -2.9599 & -0.7622 & -2.1545 & -1.5430 & -1.4365 & 1.3082 & 2.6165 & 0.7796 \\
\hline $\mathrm{H}$ & -1.2672 & -3.7699 & 1.5433 & -3.2453 & -0.9515 & 1.1486 & 2.0459 & 4.4954 & -1.4883 \\
\hline $\mathrm{H}$ & -2.1160 & -2.8769 & 0.2640 & -3.3269 & 0.5203 & 0.1718 & 0.3393 & 4.1675 & -1.1106 \\
\hline $\mathrm{H}$ & 2.7779 & -7.8267 & -3.7364 & -4.9907 & -5.0996 & -5.3232 & 4.7225 & 4.6487 & 5.6459 \\
\hline $\mathrm{H}$ & 1.5629 & -8.4357 & -2.5837 & -6.2238 & -4.5191 & -4.1749 & 5.0338 & 5.7174 & 4.2539 \\
\hline $\mathrm{H}$ & 1.0482 & -7.4368 & -3.9722 & -5.4270 & -3.3660 & -5.2801 & 3.4142 & 5.6896 & 5.0082 \\
\hline $\mathrm{H}$ & -0.3066 & 0.0859 & 0.7969 & 0.2528 & 0.3641 & -0.3849 & 0.5889 & 0.8524 & -2.3509 \\
\hline $\mathrm{H}$ & -0.5694 & -0.9377 & -0.6399 & -1.1917 & 0.4663 & -1.4246 & 0.1720 & 1.3965 & -0.6989 \\
\hline $\mathrm{H}$ & -1.9328 & -0.5530 & 0.4475 & -0.9769 & 1.6148 & -0.0721 & -0.5524 & 2.2119 & -2.1108 \\
\hline $\mathrm{H}$ & -1.1572 & -5.1540 & -3.9722 & -4.6983 & -0.3785 & -4.4854 & 0.6005 & 5.7527 & 3.5536 \\
\hline $\mathrm{H}$ & -4.5940 & -2.0059 & 0.4808 & -4.2239 & 2.7490 & 1.0649 & -1.6308 & 5.3166 & -2.5121 \\
\hline $\mathrm{C}$ & -6.1378 & -1.9087 & 2.7749 & -2.6606 & -2.7737 & 4.0231 & 5.8252 & -2.2477 & -2.4546 \\
\hline $\mathrm{C}$ & -1.3869 & 0.0416 & 3.7409 & -5.4006 & 1.3599 & 5.7833 & 4.6369 & 2.7251 & -2.5099 \\
\hline $\mathrm{C}$ & -6.5606 & 0.2487 & 4.4709 & -0.7743 & -0.7332 & 4.1172 & 8.1213 & -1.0301 & -3.4467 \\
\hline $\mathrm{C}$ & -7.1980 & -1.0346 & 2.5386 & -1.3456 & -3.0530 & 4.3981 & 7.0828 & -2.6668 & -2.0192 \\
\hline $\mathrm{C}$ & -2.7931 & 1.4056 & 5.6755 & -3.0390 & 2.6369 & 5.1359 & 7.0038 & 2.8622 & -3.9230 \\
\hline $\mathrm{C}$ & -0.9378 & 1.2918 & 4.1522 & -5.0072 & 2.4813 & 6.5071 & 5.4477 & 3.8481 & -2.3805 \\
\hline $\mathrm{C}$ & -7.4082 & 0.0511 & 3.3808 & -0.3978 & -2.0367 & 4.4451 & 8.2339 & -2.0592 & -2.5097 \\
\hline $\mathrm{C}$ & -1.6528 & 1.9839 & 5.1272 & -3.8207 & 3.1298 & 6.1765 & 6.6371 & 3.9226 & -3.0985 \\
\hline $\mathrm{C}$ & -0.7933 & -5.1086 & 4.1975 & -7.8721 & -0.1170 & 1.2432 & 0.6364 & 0.3940 & -5.1898 \\
\hline $\mathrm{C}$ & -4.2684 & -3.8854 & 3.5787 & -5.0595 & -2.2305 & 2.5303 & 3.3872 & -1.7608 & -3.8487 \\
\hline $\mathrm{C}$ & -1.7308 & -2.9035 & 4.2752 & -6.2241 & 0.3187 & 2.9090 & 2.8201 & 0.9579 & -4.3765 \\
\hline
\end{tabular}




\begin{tabular}{|c|c|c|c|c|c|c|c|c|c|}
\hline $\mathrm{C}$ & -3.1845 & -4.7825 & 4.1481 & -6.0491 & -1.6300 & 1.5434 & 2.2495 & -1.3357 & -4.7617 \\
\hline $\mathrm{C}$ & -4.5300 & -0.4045 & 5.8490 & -2.5089 & 0.9539 & 3.3442 & 6.7011 & 0.5438 & -4.8531 \\
\hline $\mathrm{C}$ & -5.2658 & -1.6952 & 3.8465 & -3.0387 & -1.4696 & 3.6877 & 5.7112 & -1.2131 & -3.3877 \\
\hline $\mathrm{C}$ & -2.5252 & -0.5576 & 4.2931 & -4.6310 & 0.8661 & 4.7258 & 4.9868 & 1.6618 & -3.3482 \\
\hline $\mathrm{C}$ & -5.5005 & -0.6176 & 4.7186 & -2.0757 & -0.4385 & 3.7245 & 6.8747 & -0.6081 & -3.8981 \\
\hline C & -3.2362 & 0.1381 & 5.2824 & -3.4281 & 1.5125 & 4.4046 & 6.1960 & 1.7306 & -4.0602 \\
\hline $\mathrm{C}$ & -2.8357 & -1.9604 & 3.7717 & -5.2358 & -0.2755 & 3.9239 & 3.9673 & 0.5352 & -3.4518 \\
\hline $\mathrm{N}$ & -1.8859 & -4.2549 & 3.7646 & -6.9679 & -0.7174 & 2.2102 & 1.7482 & -0.0297 & -4.3574 \\
\hline $\mathrm{N}$ & -4.1495 & -2.5347 & 4.1249 & -4.3214 & -1.1548 & 3.1843 & 4.4650 & -0.7761 & -3.9068 \\
\hline $\mathrm{H}$ & -5.9931 & -2.7538 & 2.1118 & -3.3895 & -3.5765 & 3.9736 & 4.9275 & -2.7148 & -2.0618 \\
\hline $\mathrm{H}$ & -0.8483 & -0.5072 & 2.9737 & -6.3300 & 0.8553 & 6.0376 & 3.7089 & 2.6654 & -1.9488 \\
\hline $\mathrm{H}$ & -6.7245 & 1.0859 & 5.1445 & -0.0300 & 0.0597 & 4.1322 & 9.0143 & -0.5510 & -3.8398 \\
\hline $\mathrm{H}$ & -7.8443 & -1.2043 & 1.6835 & -1.0647 & -4.0726 & 4.6467 & 7.1547 & -3.4674 & -1.2886 \\
\hline $\mathrm{H}$ & -3.3624 & 1.9441 & 6.4286 & -2.1034 & 3.1263 & 4.8769 & 7.9440 & 2.9009 & -4.4668 \\
\hline $\mathrm{H}$ & -0.0351 & 1.7160 & 3.7206 & -5.6235 & 2.8430 & 7.3236 & 5.1502 & 4.6489 & -1.7103 \\
\hline $\mathrm{H}$ & -8.2282 & 0.7376 & 3.1977 & 0.6333 & -2.2526 & 4.7058 & 9.2131 & -2.3826 & -2.1722 \\
\hline $\mathrm{H}$ & -1.3260 & 2.9663 & 5.4545 & -3.5001 & 4.0061 & 6.7304 & 7.2847 & 4.7890 & -3.0078 \\
\hline $\mathrm{H}$ & -0.9392 & -6.1165 & 3.7997 & -8.4437 & -0.8991 & 0.7361 & -0.1793 & -0.3320 & -5.1126 \\
\hline $\mathrm{H}$ & 0.1536 & -4.7207 & 3.8089 & -8.5717 & 0.5518 & 1.7520 & 0.2721 & 1.3648 & -4.8404 \\
\hline $\mathrm{H}$ & -0.7181 & -5.1764 & 5.2968 & -7.3290 & 0.4676 & 0.4765 & 0.9162 & 0.4908 & -6.2535 \\
\hline $\mathrm{H}$ & -5.2566 & -4.2747 & 3.8370 & -4.3538 & -2.8673 & 1.9866 & 3.7835 & -2.7255 & -4.1796 \\
\hline $\mathrm{H}$ & -4.1705 & -3.8902 & 2.4777 & -5.6049 & -2.8534 & 3.2607 & 3.0009 & -1.8891 & -2.8241 \\
\hline $\mathrm{H}$ & -0.7641 & -2.5177 & 3.9369 & -6.9329 & 0.9683 & 3.4330 & 2.4173 & 1.9099 & -4.0169 \\
\hline $\mathrm{H}$ & -1.7423 & -2.8830 & 5.3834 & -5.6432 & 0.9488 & 2.2022 & 3.2248 & 1.1033 & -5.3976 \\
\hline $\mathrm{H}$ & -3.2927 & -4.8409 & 5.2475 & -5.4842 & -1.1052 & 0.7498 & 2.6050 & -1.3210 & -5.8092 \\
\hline $\mathrm{H}$ & -3.2942 & -5.7924 & 3.7404 & -6.6245 & -2.4296 & 1.0638 & 1.4356 & -2.0665 & -4.6863 \\
\hline $\mathrm{H}$ & -4.9388 & 0.3019 & 6.5757 & -1.6469 & 1.6138 & 3.2264 & 7.6506 & 0.7937 & -5.3329 \\
\hline $\mathrm{H}$ & -4.3497 & -1.3592 & 6.3543 & -3.0334 & 0.9113 & 2.3828 & 5.9784 & 0.2640 & -5.6272 \\
\hline \multirow[t]{2}{*}{$\mathrm{H}$} & -2.7512 & -1.9202 & 2.6670 & -5.8264 & -0.8904 & 4.6271 & 3.5151 & 0.4164 & -2.4505 \\
\hline & \multicolumn{3}{|c|}{ F $\Lambda 2$} & \multicolumn{3}{|c|}{ FV2 } & \multicolumn{3}{|c|}{$\mathbf{S}$} \\
\hline atom & $\mathbf{x}$ & $\mathbf{y}$ & $\mathbf{Z}$ & $\mathbf{x}$ & $\mathbf{y}$ & $\mathbf{Z}$ & $\mathbf{x}$ & $\mathbf{y}$ & $\mathbf{Z}$ \\
\hline $\mathrm{C}$ & -4.0651 & -3.2745 & -2.5314 & -4.6914 & -1.4771 & 2.5198 & -4.1750 & 0.5147 & 1.1843 \\
\hline $\mathrm{C}$ & -4.4470 & -2.5989 & -3.8524 & -4.3813 & -2.3977 & 3.7010 & -4.3159 & -0.3203 & 2.4535 \\
\hline $\mathrm{C}$ & -4.1290 & -1.1096 & -3.8092 & -3.1079 & -3.1857 & 3.4254 & -3.2534 & -1.4057 & 2.4991 \\
\hline $\mathrm{C}$ & -4.6815 & -0.4878 & -2.5296 & -3.1887 & -3.8523 & 2.0550 & -3.3091 & -2.1997 & 1.1997 \\
\hline C & -4.2026 & -1.2587 & -1.3084 & -3.5685 & -2.8537 & 0.9698 & -3.1958 & -1.2961 & -0.0253 \\
\hline $\mathrm{C}$ & -4.6862 & -0.7169 & 0.0252 & -3.7039 & -3.4693 & -0.4155 & -3.3615 & -2.0577 & -1.3350 \\
\hline $\mathrm{C}$ & -4.4038 & -3.3034 & -6.1286 & -4.6401 & -2.1918 & 6.0605 & -5.0644 & 0.2082 & 4.6473 \\
\hline $\mathrm{C}$ & -6.9488 & -1.4434 & -0.0262 & -5.7501 & -4.6332 & -0.1859 & -5.6900 & -2.5579 & -1.2426 \\
\hline $\mathrm{O}$ & -3.7411 & -3.2785 & -4.8709 & -4.2765 & -1.5628 & 4.8379 & -4.2359 & 0.5669 & 3.5501 \\
\hline $\mathrm{O}$ & -4.6840 & -0.4669 & -4.9339 & -2.9123 & -4.1619 & 4.4227 & -3.4910 & -2.2522 & 3.5988 \\
\hline $\mathrm{O}$ & -4.1942 & 0.8399 & -2.5308 & -1.8830 & -4.3553 & 1.8203 & -2.2398 & -3.1264 & 1.2510 \\
\hline $\mathrm{O}$ & -4.6413 & -2.6153 & -1.4334 & -4.7881 & -2.1920 & 1.3152 & -4.2213 & -0.2999 & 0.0389 \\
\hline $\mathrm{O}$ & -6.0489 & -0.3508 & 0.0604 & -4.3582 & -4.7176 & -0.4332 & -4.3646 & -3.0495 & -1.3020 \\
\hline $\mathrm{H}$ & -4.4590 & -4.2960 & -2.5154 & -5.6638 & -0.9976 & 2.6707 & -5.0371 & 1.1816 & 1.1197 \\
\hline $\mathrm{H}$ & -5.5316 & -2.7232 & -3.9899 & -5.2216 & -3.0987 & 3.8158 & -5.3131 & -0.7845 & 2.4209 \\
\hline $\mathrm{H}$ & -3.0328 & -0.9982 & -3.7881 & -2.2700 & -2.4706 & 3.3996 & -2.2657 & -0.9224 & 2.5721 \\
\hline $\mathrm{H}$ & -5.7799 & -0.4920 & -2.5695 & -3.9151 & -4.6755 & 2.0974 & -4.2679 & -2.7344 & 1.1648 \\
\hline $\mathrm{H}$ & -3.1030 & -1.2310 & -1.2971 & -2.7654 & -2.1137 & 0.9316 & -2.2104 & -0.8049 & -0.0235 \\
\hline $\mathrm{H}$ & -4.1240 & 0.1885 & 0.2734 & -2.7016 & -3.6418 & -0.8181 & -2.4319 & -2.5858 & -1.5653 \\
\hline $\mathrm{H}$ & -3.7728 & -3.8824 & -6.8035 & -4.5476 & -1.4362 & 6.8410 & -4.9824 & 1.0111 & 5.3812 \\
\hline $\mathrm{H}$ & -5.3814 & -3.7924 & -6.0401 & -5.6779 & -2.5441 & 6.0201 & -6.1082 & 0.1197 & 4.3206 \\
\hline $\mathrm{H}$ & -4.5378 & -2.2918 & -6.5182 & -3.9788 & -3.0341 & 6.2755 & -4.7375 & -0.7374 & 5.0863 \\
\hline $\mathrm{H}$ & -7.9298 & -1.0741 & 0.2767 & -6.1745 & -5.6146 & -0.4026 & -6.3542 & -3.3741 & -1.5346 \\
\hline $\mathrm{H}$ & -7.0052 & -1.8441 & -1.0436 & -5.9654 & -4.3645 & 0.8543 & -5.9623 & -2.2288 & -0.2305 \\
\hline $\mathrm{H}$ & -6.6418 & -2.2575 & 0.6445 & -6.2169 & -3.8830 & -0.8387 & -5.8356 & -1.7089 & -1.9257 \\
\hline
\end{tabular}




\begin{tabular}{|c|c|c|c|c|c|c|c|c|c|}
\hline $\mathrm{H}$ & -4.6721 & 0.4908 & -4.7526 & -2.2328 & -4.7780 & 4.0922 & -2.9676 & -3.0637 & 3.4643 \\
\hline $\mathrm{C}$ & -5.0236 & 1.8612 & -2.0443 & -1.7450 & -5.5895 & 1.1688 & -2.4914 & -4.4498 & 0.8539 \\
\hline $\mathrm{C}$ & -4.9727 & 2.9934 & -3.0764 & -0.7957 & -6.4419 & 2.0184 & -1.9113 & -5.3644 & 1.9367 \\
\hline $\mathrm{C}$ & -3.5779 & 3.6061 & -3.1024 & 0.6078 & -5.8521 & 1.9797 & -0.3934 & -5.2530 & 1.9532 \\
\hline $\mathrm{C}$ & -3.1071 & 3.9413 & -1.6878 & 1.0432 & -5.5852 & 0.5393 & 0.1721 & -5.4345 & 0.5458 \\
\hline $\mathrm{C}$ & -3.2358 & 2.7317 & -0.7697 & 0.0066 & -4.7407 & -0.1933 & -0.5134 & -4.5026 & -0.4503 \\
\hline $\mathrm{C}$ & -2.8518 & 3.0184 & 0.6624 & 0.3133 & -4.5117 & -1.6676 & -0.0941 & -4.7605 & -1.8859 \\
\hline $\mathrm{C}$ & -5.9260 & 3.3033 & -5.2429 & -1.0420 & -7.6156 & 4.0826 & -2.6338 & -5.9886 & 4.1262 \\
\hline $\mathrm{C}$ & -1.9453 & 2.0189 & 2.5611 & 0.6873 & -2.2116 & -1.9876 & 0.3361 & -2.6235 & -2.7719 \\
\hline $\mathrm{O}$ & -5.3305 & 2.4094 & -4.3132 & -1.3405 & -6.4525 & 3.3231 & -2.4938 & -4.9578 & 3.1586 \\
\hline $\mathrm{O}$ & -3.5792 & 4.7666 & -3.9017 & 1.5124 & -6.7309 & 2.6076 & 0.1409 & -6.2224 & 2.8228 \\
\hline $\mathrm{O}$ & -1.7438 & 4.3274 & -1.7931 & 2.2727 & -4.8775 & 0.6127 & 1.5585 & -5.1386 & 0.6387 \\
\hline $\mathrm{O}$ & -4.5964 & 2.2999 & -0.7826 & -1.2496 & -5.4170 & -0.1328 & -1.9208 & -4.7251 & -0.3955 \\
\hline $\mathrm{O}$ & -2.4541 & 1.8063 & 1.2618 & -0.2267 & -3.2821 & -2.1198 & -0.5272 & -3.7392 & -2.7600 \\
\hline $\mathrm{H}$ & -6.0465 & 1.4979 & -1.9105 & -2.7172 & -6.0776 & 1.0584 & -3.5659 & -4.6176 & 0.7389 \\
\hline $\mathrm{H}$ & -5.7034 & 3.7642 & -2.7905 & -0.7728 & -7.4609 & 1.6051 & -2.1950 & -6.4015 & 1.7054 \\
\hline $\mathrm{H}$ & -2.8893 & 2.8475 & -3.5058 & 0.5748 & -4.8804 & 2.4970 & -0.1356 & -4.2344 & 2.2842 \\
\hline $\mathrm{H}$ & -3.7115 & 4.7680 & -1.2874 & 1.1780 & -6.5407 & 0.0119 & 0.0230 & -6.4786 & 0.2329 \\
\hline $\mathrm{H}$ & -2.5840 & 1.9293 & -1.1409 & -0.0683 & -3.7658 & 0.3110 & -0.2894 & -3.4633 & -0.1706 \\
\hline $\mathrm{H}$ & -3.7050 & 3.4614 & 1.1979 & -0.1563 & -5.3136 & -2.2446 & -0.5664 & -5.6903 & -2.2191 \\
\hline $\mathrm{H}$ & -2.0260 & 3.7440 & 0.6804 & 1.3968 & -4.5514 & -1.8410 & 0.9959 & -4.8867 & -1.9377 \\
\hline $\mathrm{H}$ & -6.2044 & 2.7087 & -6.1138 & -1.5646 & -7.5100 & 5.0341 & -3.1411 & -5.5451 & 4.9838 \\
\hline $\mathrm{H}$ & -6.8281 & 3.7580 & -4.8156 & -1.4074 & -8.5137 & 3.5702 & -3.2468 & -6.8069 & 3.7298 \\
\hline $\mathrm{H}$ & -5.2278 & 4.0910 & -5.5347 & 0.0327 & -7.7080 & 4.2541 & -1.6592 & -6.3781 & 4.4281 \\
\hline $\mathrm{H}$ & -1.5766 & 1.0601 & 2.9339 & 0.1392 & -1.2813 & -2.1546 & -0.0457 & -1.9244 & -3.5183 \\
\hline $\mathrm{H}$ & -1.1135 & 2.7382 & 2.5323 & 1.1252 & -2.1737 & -0.9802 & 0.3765 & -2.1142 & -1.7986 \\
\hline $\mathrm{H}$ & -2.7185 & 2.3998 & 3.2382 & 1.4987 & -2.2918 & -2.7239 & 1.3576 & -2.9216 & -3.0493 \\
\hline $\mathrm{H}$ & -2.6598 & 5.0892 & -3.9477 & 2.3817 & -6.2896 & 2.6316 & 1.1129 & -6.1627 & 2.7685 \\
\hline $\mathrm{C}$ & -1.4265 & 5.6636 & -1.5214 & 3.4319 & -5.5320 & 0.1766 & 2.4826 & -6.1094 & 0.2329 \\
\hline $\mathrm{C}$ & -0.3776 & 6.1105 & -2.5441 & 4.5641 & -5.1723 & 1.1426 & 3.6029 & -6.1461 & 1.2769 \\
\hline $\mathrm{C}$ & 0.9321 & 5.3698 & -2.3160 & 4.8722 & -3.6840 & 1.0588 & 4.3877 & -4.8424 & 1.2543 \\
\hline $\mathrm{C}$ & 1.3392 & 5.5140 & -0.8553 & 5.0810 & -3.2733 & -0.3928 & 4.8258 & -4.5437 & -0.1734 \\
\hline $\mathrm{C}$ & 0.2241 & 5.0698 & 0.0859 & 3.9468 & -3.7526 & -1.2933 & 3.6446 & -4.5569 & -1.1400 \\
\hline $\mathrm{C}$ & 0.5804 & 5.2746 & 1.5532 & 4.1854 & -3.4482 & -2.7657 & 4.0685 & -4.3257 & -2.5852 \\
\hline $\mathrm{C}$ & -0.5049 & 6.7876 & -4.8278 & 5.1942 & -5.9607 & 3.3036 & 3.7860 & -7.1343 & 3.4413 \\
\hline $\mathrm{C}$ & 0.3959 & 7.6352 & 1.7442 & 5.7858 & -5.1187 & -3.3134 & 5.0916 & -6.4036 & -3.1085 \\
\hline $\mathrm{O}$ & -0.9186 & 5.8641 & -3.8277 & 4.1411 & -5.5570 & 2.4364 & 2.9946 & -6.3816 & 2.5307 \\
\hline $\mathrm{O}$ & 1.9277 & 5.9050 & -3.1558 & 6.0258 & -3.3909 & 1.8145 & 5.5100 & -4.9570 & 2.0973 \\
\hline $\mathrm{O}$ & 2.4964 & 4.7136 & -0.6871 & 5.1301 & -1.8553 & -0.3728 & 5.4490 & -3.2725 & -0.1362 \\
\hline $\mathrm{O}$ & -0.9597 & 5.8207 & -0.2046 & 3.7577 & -5.1610 & -1.1395 & 2.9931 & -5.8289 & -1.0472 \\
\hline $\mathrm{O}$ & 1.2335 & 6.4960 & 1.8207 & 5.4887 & -3.7364 & -3.2210 & 5.2482 & -5.0046 & -2.9551 \\
\hline $\mathrm{H}$ & -2.3162 & 6.2964 & -1.6039 & 3.2841 & -6.6167 & 0.1627 & 2.0056 & -7.0923 & 0.1605 \\
\hline $\mathrm{H}$ & -0.2048 & 7.1879 & -2.4050 & 5.4567 & -5.7429 & 0.8469 & 4.2804 & -6.9740 & 1.0214 \\
\hline $\mathrm{H}$ & 0.7639 & 4.3014 & -2.5261 & 3.9964 & -3.1373 & 1.4423 & 3.7187 & -4.0335 & 1.5885 \\
\hline $\mathrm{H}$ & 1.5785 & 6.5688 & -0.6622 & 6.0391 & -3.6840 & -0.7403 & 5.5506 & -5.3096 & -0.4819 \\
\hline $\mathrm{H}$ & 0.0287 & 3.9985 & -0.0779 & 3.0336 & -3.2290 & -0.9830 & 2.9354 & -3.7634 & -0.8570 \\
\hline $\mathrm{H}$ & 1.2803 & 4.4924 & 1.8618 & 4.0445 & -2.3734 & -2.9161 & 4.2814 & -3.2612 & -2.7248 \\
\hline $\mathrm{H}$ & -0.3424 & 5.1880 & 2.1486 & 3.4365 & -3.9942 & -3.3603 & 3.2331 & -4.6147 & -3.2428 \\
\hline $\mathrm{H}$ & -1.0135 & 6.4996 & -5.7484 & 4.7292 & -6.2521 & 4.2458 & 3.1878 & -7.2616 & 4.3441 \\
\hline $\mathrm{H}$ & -0.7998 & 7.8082 & -4.5563 & 5.7306 & -6.8201 & 2.8843 & 4.0218 & -8.1198 & 3.0228 \\
\hline $\mathrm{H}$ & 0.5770 & 6.7453 & -4.9691 & 5.8945 & -5.1394 & 3.4702 & 4.7124 & -6.6068 & 3.6780 \\
\hline $\mathrm{H}$ & 0.9501 & 8.4684 & 2.1796 & 6.7116 & -5.2073 & -3.8837 & 5.9974 & -6.7750 & -3.5904 \\
\hline $\mathrm{H}$ & 0.1266 & 7.8780 & 0.7105 & 5.9200 & -5.5746 & -2.3268 & 4.9574 & -6.9097 & -2.1463 \\
\hline $\mathrm{H}$ & -0.5296 & 7.4790 & 2.3134 & 4.9842 & -5.6557 & -3.8363 & 4.2246 & -6.6324 & -3.7419 \\
\hline $\mathrm{H}$ & 2.7918 & 5.6185 & -2.8069 & 6.3807 & -2.5447 & 1.4862 & 6.1330 & -4.2468 & 1.8565 \\
\hline $\mathrm{C}$ & 3.5491 & 5.2300 & 0.0875 & 5.9719 & -1.2185 & -1.3024 & 6.6225 & -3.0932 & -0.8893 \\
\hline
\end{tabular}




\begin{tabular}{|c|c|c|c|c|c|c|c|c|c|}
\hline $\mathrm{C}$ & 4.8341 & 5.0925 & -0.7355 & 7.0144 & -0.4157 & -0.5153 & 7.6843 & -2.5068 & 0.0468 \\
\hline $\mathrm{C}$ & 5.2167 & 3.6260 & -0.8898 & 6.3601 & 0.7717 & 0.1749 & 7.3157 & -1.0841 & 0.4453 \\
\hline $\mathrm{C}$ & 5.2001 & 2.9325 & 0.4709 & 5.4955 & 1.5525 & -0.8130 & 6.9659 & -0.2628 & -0.7942 \\
\hline $\mathrm{C}$ & 3.8606 & 3.1493 & 1.1732 & 4.4838 & 0.6306 & -1.4869 & 5.8956 & -0.9643 & -1.6274 \\
\hline $\mathrm{C}$ & 3.8367 & 2.5522 & 2.5667 & 3.6191 & 1.3211 & -2.5371 & 5.5888 & -0.2322 & -2.9189 \\
\hline $\mathrm{C}$ & 5.7223 & 6.2533 & -2.6281 & 8.9319 & -1.0425 & 0.7661 & 9.0144 & -3.4079 & 1.8183 \\
\hline $\mathrm{C}$ & 1.6122 & 1.7752 & 2.7375 & 1.4489 & 1.3687 & -1.6681 & 3.2506 & -0.4532 & -3.1328 \\
\hline $\mathrm{O}$ & 4.5781 & 5.7212 & -1.9749 & 7.5876 & -1.3254 & 0.4035 & 7.7587 & -3.3814 & 1.1543 \\
\hline $\mathrm{O}$ & 6.4961 & 3.5387 & -1.4697 & 7.3549 & 1.6043 & 0.7258 & 8.3957 & -0.4968 & 1.1312 \\
\hline $\mathrm{O}$ & 5.4494 & 1.5568 & 0.2180 & 4.8298 & 2.5653 & -0.0711 & 6.5200 & 1.0022 & -0.3243 \\
\hline $\mathrm{O}$ & 3.6559 & 4.5541 & 1.3103 & 5.2186 & -0.3999 & -2.1553 & 6.3920 & -2.2529 & -1.9857 \\
\hline $\mathrm{O}$ & 2.5876 & 2.6867 & 3.2098 & 2.3195 & 0.7624 & -2.6062 & 4.5224 & -0.8138 & -3.6399 \\
\hline $\mathrm{H}$ & 3.3606 & 6.2775 & 0.3376 & 6.4587 & -1.9581 & -1.9444 & 6.9580 & -4.0468 & -1.3051 \\
\hline $\mathrm{H}$ & 5.6437 & 5.6150 & -0.2049 & 7.7786 & -0.0473 & -1.2156 & 8.6460 & -2.4876 & -0.4870 \\
\hline $\mathrm{H}$ & 4.4558 & 3.1407 & -1.5223 & 5.6928 & 0.3795 & 0.9587 & 6.4162 & -1.1317 & 1.0800 \\
\hline $\mathrm{H}$ & 6.0029 & 3.3550 & 1.0934 & 6.1331 & 2.0033 & -1.5878 & 7.8711 & -0.1424 & -1.4078 \\
\hline $\mathrm{H}$ & 3.0596 & 2.7138 & 0.5593 & 3.8342 & 0.1841 & -0.7211 & 4.9832 & -1.0711 & -1.0245 \\
\hline $\mathrm{H}$ & 4.5691 & 3.0810 & 3.1876 & 4.0937 & 1.1816 & -3.5136 & 6.4728 & -0.2811 & -3.5651 \\
\hline $\mathrm{H}$ & 4.1250 & 1.4960 & 2.5016 & 3.5594 & 2.3974 & -2.3328 & 5.3745 & 0.8197 & -2.6955 \\
\hline $\mathrm{H}$ & 5.3599 & 6.7758 & -3.5142 & 9.2522 & -1.8485 & 1.4274 & 8.9459 & -4.1827 & 2.5830 \\
\hline $\mathrm{H}$ & 6.2389 & 6.9676 & -1.9758 & 9.5746 & -1.0300 & -0.1224 & 9.8149 & -3.6668 & 1.1149 \\
\hline $\mathrm{H}$ & 6.4154 & 5.4606 & -2.9174 & 9.0071 & -0.0826 & 1.2815 & 9.2365 & -2.4436 & 2.2801 \\
\hline $\mathrm{H}$ & 0.8030 & 1.7641 & 3.4704 & 0.4900 & 0.8494 & -1.6992 & 2.5086 & -0.8044 & -3.8532 \\
\hline $\mathrm{H}$ & 1.2007 & 2.0785 & 1.7631 & 1.8574 & 1.3141 & -0.6465 & 3.0447 & -0.9249 & -2.1613 \\
\hline $\mathrm{H}$ & 2.0307 & 0.7639 & 2.6529 & 1.2862 & 2.4240 & -1.9273 & 3.1713 & 0.6362 & -3.0296 \\
\hline $\mathrm{H}$ & 6.7819 & 2.6076 & -1.4219 & 6.9062 & 2.2935 & 1.2503 & 8.1657 & 0.4351 & 1.3040 \\
\hline $\mathrm{C}$ & 6.4620 & 0.9135 & 0.9440 & 5.2933 & 3.8767 & -0.2481 & 7.1917 & 2.1466 & -0.7740 \\
\hline $\mathrm{C}$ & 7.2999 & 0.0964 & -0.0440 & 5.2703 & 4.5887 & 1.1076 & 7.3940 & 3.0792 & 0.4238 \\
\hline $\mathrm{C}$ & 6.4690 & -1.0434 & -0.6114 & 3.8357 & 4.7599 & 1.5778 & 6.0573 & 3.6256 & 0.8989 \\
\hline $\mathrm{C}$ & 5.8214 & -1.8426 & 0.5172 & 2.9717 & 5.3849 & 0.4842 & 5.2857 & 4.2250 & -0.2741 \\
\hline $\mathrm{C}$ & 5.0492 & -0.9292 & 1.4686 & 3.1244 & 4.6354 & -0.8398 & 5.1686 & 3.2225 & -1.4212 \\
\hline $\mathrm{C}$ & 4.4974 & -1.6563 & 2.6812 & 2.4019 & 5.2878 & -2.0056 & 4.5099 & 3.8048 & -2.6580 \\
\hline $\mathrm{C}$ & 9.0076 & 0.6754 & -1.6084 & 6.6988 & 4.5528 & 3.0174 & 8.9479 & 3.0865 & 2.2351 \\
\hline $\mathrm{C}$ & 4.0712 & -0.1855 & 4.5085 & 3.4187 & 4.5644 & -4.0345 & 5.0919 & 2.5870 & -4.6157 \\
\hline $\mathrm{O}$ & 7.7385 & 0.9868 & -1.0488 & 6.0352 & 3.8060 & 2.0040 & 8.0487 & 2.3342 & 1.4303 \\
\hline $\mathrm{O}$ & 7.2893 & -1.8785 & -1.3918 & 3.7944 & 5.5637 & 2.7341 & 6.2796 & 4.5988 & 1.8899 \\
\hline $\mathrm{O}$ & 4.9559 & -2.7700 & -0.1212 & 1.6579 & 5.2747 & 1.0083 & 4.0062 & 4.5667 & 0.2391 \\
\hline $\mathrm{O}$ & 5.9348 & 0.0853 & 1.9493 & 4.5099 & 4.5693 & -1.1846 & 6.4810 & 2.7967 & -1.7970 \\
\hline $\mathrm{O}$ & 3.5561 & -0.8563 & 3.3709 & 2.3310 & 4.4410 & -3.1355 & 4.1507 & 2.7930 & -3.5767 \\
\hline $\mathrm{H}$ & 7.0943 & 1.6461 & 1.4558 & 6.3138 & 3.8739 & -0.6441 & 8.1622 & 1.8787 & -1.2041 \\
\hline $\mathrm{H}$ & 8.1610 & -0.3189 & 0.4993 & 5.7315 & 5.5781 & 0.9756 & 8.0276 & 3.9164 & 0.0973 \\
\hline $\mathrm{H}$ & 5.6589 & -0.6024 & -1.2137 & 3.4303 & 3.7552 & 1.7778 & 5.4662 & 2.7825 & 1.2908 \\
\hline $\mathrm{H}$ & 6.6072 & -2.3737 & 1.0745 & 3.2506 & 6.4426 & 0.3643 & 5.8107 & 5.1259 & -0.6245 \\
\hline $\mathrm{H}$ & 4.2127 & -0.4664 & 0.9251 & 2.7282 & 3.6221 & -0.6972 & 4.5788 & 2.3582 & -1.0818 \\
\hline $\mathrm{H}$ & 5.3269 & -1.9484 & 3.3398 & 2.9005 & 6.2363 & -2.2559 & 5.1868 & 4.5379 & -3.1193 \\
\hline $\mathrm{H}$ & 3.9917 & -2.5659 & 2.3465 & 1.3724 & 5.4989 & -1.7049 & 3.5980 & 4.3270 & -2.3558 \\
\hline $\mathrm{H}$ & 9.2272 & 1.4557 & -2.3378 & 7.2683 & 3.8370 & 3.6107 & 9.3939 & 2.3869 & 2.9428 \\
\hline $\mathrm{H}$ & 9.7817 & 0.6799 & -0.8320 & 7.3863 & 5.2814 & 2.5718 & 9.7390 & 3.5291 & 1.6184 \\
\hline $\mathrm{H}$ & 8.9876 & -0.3002 & -2.0988 & 5.9759 & 5.0719 & 3.6501 & 8.4183 & 3.8757 & 2.7729 \\
\hline $\mathrm{H}$ & 3.3271 & 0.5626 & 4.7977 & 3.1926 & 3.9282 & -4.8925 & 4.7842 & 1.6815 & -5.1440 \\
\hline $\mathrm{H}$ & 4.2281 & -0.8862 & 5.3380 & 3.5237 & 5.6021 & -4.3769 & 5.0947 & 3.4339 & -5.3136 \\
\hline $\mathrm{H}$ & 5.0142 & 0.3216 & 4.2791 & 4.3616 & 4.2427 & -3.5813 & 6.1022 & 2.4441 & -4.2189 \\
\hline $\mathrm{H}$ & 6.7519 & -2.6435 & -1.6692 & 2.8605 & 5.7983 & 2.8856 & 5.4191 & 5.0048 & 2.1043 \\
\hline $\mathrm{C}$ & 5.0841 & -4.1318 & 0.1812 & 0.7155 & 6.2764 & 0.7558 & 3.5360 & 5.8755 & 0.0662 \\
\hline $\mathrm{C}$ & 4.9887 & -4.9116 & -1.1351 & 0.0311 & 6.6023 & 2.0894 & 2.9222 & 6.3276 & 1.3957 \\
\hline $\mathrm{C}$ & 3.5850 & -4.8023 & -1.7150 & -0.8404 & 5.4329 & 2.5378 & 1.6682 & 5.5206 & 1.7008 \\
\hline
\end{tabular}




\begin{tabular}{|c|c|c|c|c|c|c|c|c|c|}
\hline $\mathrm{C}$ & 2.5585 & -5.1526 & -0.6447 & -1.7085 & 4.9261 & 1.3880 & 0.7407 & 5.5426 & 0.4927 \\
\hline $\mathrm{C}$ & 2.7607 & -4.3183 & 0.6169 & -0.8572 & 4.6150 & 0.1611 & 1.4587 & 5.0974 & -0.7774 \\
\hline $\mathrm{C}$ & 1.7800 & -4.6605 & 1.7295 & -1.6136 & 4.0774 & -1.0429 & 0.5706 & 5.1651 & -2.0125 \\
\hline $\mathrm{C}$ & 6.4952 & -5.2980 & -2.9472 & 0.6950 & 7.6973 & 4.1030 & 3.8457 & 7.0893 & 3.4618 \\
\hline $\mathrm{C}$ & 2.6058 & -6.7628 & 2.4573 & -2.7487 & 5.9558 & -1.9270 & 0.4564 & 7.5087 & -2.3763 \\
\hline $\mathrm{O}$ & 5.9747 & -4.3791 & -1.9953 & 1.0751 & 6.8926 & 2.9955 & 3.9202 & 6.1666 & 2.3827 \\
\hline $\mathrm{O}$ & 3.4533 & -5.6781 & -2.8113 & -1.6674 & 5.8299 & 3.6099 & 1.0162 & 6.0683 & 2.8232 \\
\hline $\mathrm{O}$ & 1.2963 & -4.9193 & -1.2437 & -2.3689 & 3.7949 & 1.9254 & -0.3301 & 4.6736 & 0.8158 \\
\hline $\mathrm{O}$ & 4.0946 & -4.5471 & 1.0884 & -0.2202 & 5.8483 & -0.2019 & 2.5949 & 5.9474 & -0.9752 \\
\hline $\mathrm{O}$ & 1.5345 & -6.0416 & 1.8770 & -2.8548 & 4.7029 & -1.2775 & -0.2436 & 6.3157 & -2.0740 \\
\hline $\mathrm{H}$ & 6.0457 & -4.3323 & 0.6649 & 1.1958 & 7.1710 & 0.3448 & 4.3540 & 6.5465 & -0.2150 \\
\hline $\mathrm{H}$ & 5.2025 & -5.9691 & -0.9199 & -0.6091 & 7.4854 & 1.9454 & 2.6484 & 7.3888 & 1.3013 \\
\hline $\mathrm{H}$ & 3.4217 & -3.7560 & -2.0190 & -0.1702 & 4.6111 & 2.8378 & 1.9699 & 4.4771 & 1.8846 \\
\hline $\mathrm{H}$ & 2.6642 & -6.2168 & -0.3919 & -2.4424 & 5.6998 & 1.1209 & 0.3676 & 6.5670 & 0.3560 \\
\hline $\mathrm{H}$ & 2.6399 & -3.2528 & 0.3717 & -0.0890 & 3.8732 & 0.4208 & 1.7989 & 4.0581 & -0.6561 \\
\hline $\mathrm{H}$ & 0.8165 & -4.2029 & 1.4929 & -1.8256 & 3.0179 & -0.8794 & -0.1169 & 4.3148 & -1.9980 \\
\hline $\mathrm{H}$ & 2.1571 & -4.2288 & 2.6699 & -0.9591 & 4.1627 & -1.9235 & 1.2103 & 5.0914 & -2.9056 \\
\hline $\mathrm{H}$ & 7.2788 & -4.7715 & -3.4929 & 1.6084 & 7.9178 & 4.6565 & 4.6949 & 6.8779 & 4.1124 \\
\hline $\mathrm{H}$ & 6.9301 & -6.1689 & -2.4426 & 0.2463 & 8.6372 & 3.7594 & 3.9217 & 8.1190 & 3.0929 \\
\hline $\mathrm{H}$ & 5.7160 & -5.6289 & -3.6370 & -0.0159 & 7.1749 & 4.7471 & 2.9115 & 6.9681 & 4.0144 \\
\hline $\mathrm{H}$ & 2.2268 & -7.7565 & 2.7016 & -3.7613 & 6.2694 & -2.1862 & -0.2923 & 8.2704 & -2.6002 \\
\hline $\mathrm{H}$ & 3.4565 & -6.8559 & 1.7735 & -2.2848 & 6.7106 & -1.2832 & 1.0772 & 7.8440 & -1.5383 \\
\hline $\mathrm{H}$ & 2.9546 & -6.2728 & 3.3759 & -2.1531 & 5.8662 & -2.8462 & 1.1048 & 7.3704 & -3.2513 \\
\hline $\mathrm{H}$ & 2.5000 & -5.7806 & -2.9870 & -2.3467 & 5.1389 & 3.7133 & 0.1075 & 5.7155 & 2.8355 \\
\hline $\mathrm{C}$ & 0.2576 & -5.8198 & -0.9607 & -3.6824 & 3.5433 & 1.5102 & -1.6305 & 5.0602 & 0.4538 \\
\hline $\mathrm{C}$ & -0.3901 & -6.2128 & -2.2962 & -4.4854 & 3.1180 & 2.7513 & -2.5112 & 4.9100 & 1.6969 \\
\hline $\mathrm{C}$ & -1.1617 & -5.0372 & -2.8823 & -4.1013 & 1.7093 & 3.2012 & -2.6505 & 3.4401 & 2.0694 \\
\hline $\mathrm{C}$ & -2.0736 & -4.4198 & -1.8233 & -4.0782 & 0.7671 & 1.9980 & -3.0345 & 2.6086 & 0.8471 \\
\hline $\mathrm{C}$ & -1.2581 & -4.0423 & -0.5916 & -3.1180 & 1.3274 & 0.9589 & -2.0990 & 2.8761 & -0.3282 \\
\hline $\mathrm{C}$ & -2.0470 & -3.4145 & 0.5465 & -2.8368 & 0.4612 & -0.2651 & -2.5513 & 2.1854 & -1.6015 \\
\hline $\mathrm{C}$ & 0.2877 & -7.5784 & -4.1339 & -5.2799 & 4.2729 & 4.6802 & -2.7719 & 6.1771 & 3.7064 \\
\hline $\mathrm{C}$ & -1.0512 & -1.2876 & 0.8066 & -0.8188 & -0.5760 & 0.3977 & -0.5483 & 1.3599 & -2.5301 \\
\hline $\mathrm{O}$ & 0.6650 & -6.6473 & -3.1293 & -4.2344 & 4.0998 & 3.7346 & -1.8905 & 5.6695 & 2.7142 \\
\hline $\mathrm{O}$ & -1.9254 & -5.4638 & -3.9872 & -5.0162 & 1.2491 & 4.1716 & -3.6292 & 3.3073 & 3.0746 \\
\hline $\mathrm{O}$ & -2.6681 & -3.2842 & -2.4348 & -3.6728 & -0.5111 & 2.4575 & -2.9806 & 1.2450 & 1.2439 \\
\hline $\mathrm{O}$ & -0.6760 & -5.2451 & -0.0872 & -3.7042 & 2.5543 & 0.5162 & -2.1131 & 4.2754 & -0.6023 \\
\hline $\mathrm{O}$ & -1.2212 & -2.5862 & 1.3456 & -1.4461 & 0.3234 & -0.4933 & -1.6065 & 2.2899 & -2.6433 \\
\hline $\mathrm{H}$ & 0.6460 & -6.7032 & -0.4474 & -4.1206 & 4.4369 & 1.0570 & -1.6384 & 6.0919 & 0.0924 \\
\hline $\mathrm{H}$ & -1.0925 & -7.0388 & -2.1088 & -5.5519 & 3.1128 & 2.4782 & -3.5077 & 5.3171 & 1.4700 \\
\hline $\mathrm{H}$ & -0.4306 & -4.2680 & -3.1792 & -3.0800 & 1.7500 & 3.6124 & -1.6687 & 3.0857 & 2.4204 \\
\hline $\mathrm{H}$ & -2.8459 & -5.1468 & -1.5339 & -5.0804 & 0.7067 & 1.5491 & -4.0597 & 2.8792 & 0.5496 \\
\hline $\mathrm{H}$ & -0.4613 & -3.3495 & -0.8951 & -2.1529 & 1.5447 & 1.4361 & -1.0828 & 2.5583 & -0.0585 \\
\hline $\mathrm{H}$ & -2.4343 & -4.2068 & 1.1935 & -3.2443 & 0.9535 & -1.1488 & -3.4653 & 2.6809 & -1.9489 \\
\hline $\mathrm{H}$ & -2.9004 & -2.8525 & 0.1562 & -3.3274 & -0.5180 & -0.1716 & -2.7853 & 1.1329 & -1.3842 \\
\hline $\mathrm{H}$ & 1.2052 & -7.8790 & -4.6411 & -4.9870 & 5.1048 & 5.3217 & -2.1691 & 6.7964 & 4.3715 \\
\hline $\mathrm{H}$ & -0.1796 & -8.4622 & -3.6827 & -6.2203 & 4.5237 & 4.1740 & -3.5513 & 6.7966 & 3.2465 \\
\hline $\mathrm{H}$ & -0.4054 & -7.1286 & -4.8482 & -5.4236 & 3.3713 & 5.2799 & -3.2396 & 5.3668 & 4.2699 \\
\hline $\mathrm{H}$ & -0.2893 & -0.7813 & 1.4041 & 0.2525 & -0.3646 & 0.3854 & 0.0780 & 1.4725 & -3.4165 \\
\hline $\mathrm{H}$ & -0.7127 & -1.3184 & -0.2374 & -1.1922 & -0.4652 & 1.4249 & 0.0692 & 1.5374 & -1.6399 \\
\hline $\mathrm{H}$ & -1.9816 & -0.7044 & 0.8606 & -0.9781 & -1.6145 & 0.0730 & -0.9345 & 0.3321 & -2.4880 \\
\hline $\mathrm{H}$ & -2.4200 & -4.6902 & -4.3146 & -4.6975 & 0.3830 & 4.4855 & -3.7377 & 2.3562 & 3.2680 \\
\hline $\mathrm{H}$ & -4.4757 & -1.4853 & 0.7873 & -4.2268 & -2.7468 & -1.0636 & -3.5661 & -1.3246 & -2.1320 \\
\hline $\mathrm{C}$ & -5.3278 & 1.3332 & 3.1631 & -2.6579 & 2.7743 & -4.0232 & -9.0641 & -2.6318 & -0.5635 \\
\hline $\mathrm{C}$ & -0.8366 & -1.2058 & 4.2399 & -5.4032 & -1.3552 & -5.7840 & -6.0494 & 1.1899 & -2.3051 \\
\hline $\mathrm{C}$ & -4.3416 & 2.6996 & 5.3815 & -0.7743 & 0.7313 & -4.1185 & -10.2826 & -1.8175 & -2.9254 \\
\hline $\mathrm{C}$ & -5.4598 & 2.7199 & 3.2496 & -1.3427 & 3.0519 & -4.3988 & -9.6011 & -3.5794 & -1.4357 \\
\hline
\end{tabular}




\begin{tabular}{|c|c|c|c|c|c|c|c|c|c|}
\hline $\mathrm{C}$ & -1.0060 & 0.3954 & 6.4704 & -3.0433 & -2.6355 & -5.1369 & -8.1644 & 1.5397 & -4.0440 \\
\hline $\mathrm{C}$ & 0.3321 & -0.8001 & 4.8754 & -5.0114 & -2.4771 & -6.5081 & -5.8052 & 1.6572 & -3.5925 \\
\hline $\mathrm{C}$ & -4.9722 & 3.4070 & 4.3571 & -0.3963 & 2.0343 & -4.4465 & -10.2075 & -3.1774 & -2.6203 \\
\hline $\mathrm{C}$ & 0.2463 & 0.0117 & 6.0037 & -3.8257 & -3.1271 & -6.1776 & -6.8735 & 1.8453 & -4.4654 \\
\hline $\mathrm{C}$ & -4.3380 & -4.9260 & 3.3373 & -7.8726 & 0.1237 & -1.2435 & -7.0782 & 2.5562 & 2.7256 \\
\hline $\mathrm{C}$ & -5.6929 & -1.4562 & 3.4855 & -5.0573 & 2.2338 & -2.5301 & -8.5507 & -0.6346 & 1.4096 \\
\hline $\mathrm{C}$ & -3.3177 & -2.8746 & 4.0198 & -6.2252 & -0.3137 & -2.9093 & -7.4053 & 1.7643 & 0.4792 \\
\hline $\mathrm{C}$ & -5.6575 & -2.9578 & 3.6831 & -6.0477 & 1.6344 & -1.5434 & -8.4371 & 0.6080 & 2.2799 \\
\hline C & -3.5090 & 0.5093 & 6.3729 & -2.5109 & -0.9536 & -3.3450 & -9.8091 & 0.6154 & -2.3681 \\
\hline $\mathrm{C}$ & -4.7098 & 0.6230 & 4.1976 & -3.0375 & 1.4706 & -3.6879 & -9.1314 & -1.2693 & -0.8699 \\
\hline C & -2.1059 & -0.8170 & 4.6931 & -4.6329 & -0.8627 & -4.7264 & -7.3470 & 0.9074 & -1.8631 \\
\hline $\mathrm{C}$ & -4.2097 & 1.3157 & 5.3131 & -2.0759 & 0.4383 & -3.7251 & -9.7641 & -0.8602 & -2.0605 \\
\hline $\mathrm{C}$ & -2.1882 & -0.0084 & 5.8387 & -3.4308 & -1.5108 & -4.4054 & -8.4182 & 1.0703 & -2.7525 \\
\hline $\mathrm{C}$ & -3.2865 & -1.3468 & 3.8741 & -5.2362 & 0.2794 & -3.9242 & -7.4718 & 0.5091 & -0.4015 \\
\hline $\mathrm{N}$ & -4.3768 & -3.4763 & 3.2255 & -6.9676 & 0.7232 & -2.2103 & -7.2747 & 1.3892 & 1.8803 \\
\hline $\mathrm{N}$ & -4.6112 & -0.7998 & 4.2206 & -4.3205 & 1.1573 & -3.1843 & -8.6663 & -0.2555 & 0.0052 \\
\hline $\mathrm{H}$ & -5.7128 & 0.8173 & 2.2876 & -3.3856 & 3.5781 & -3.9731 & -8.5882 & -2.9599 & 0.3548 \\
\hline $\mathrm{H}$ & -0.7712 & -1.8408 & 3.3613 & -6.3320 & -0.8494 & -6.0381 & -5.2202 & 1.0137 & -1.6230 \\
\hline $\mathrm{H}$ & -3.9557 & 3.2256 & 6.2507 & -0.0309 & -0.0625 & -4.1342 & -10.7647 & -1.4915 & -3.8433 \\
\hline $\mathrm{H}$ & -5.9474 & 3.2591 & 2.4428 & -1.0606 & 4.0711 & -4.6474 & -9.5382 & -4.6330 & -1.1829 \\
\hline $\mathrm{H}$ & -1.0797 & 1.0327 & 7.3476 & -2.1083 & -3.1262 & -4.8782 & -8.9981 & 1.6549 & -4.7313 \\
\hline $\mathrm{H}$ & 1.2981 & -1.1040 & 4.4789 & -5.6282 & -2.8377 & -7.3246 & -4.7872 & 1.8603 & -3.9124 \\
\hline $\mathrm{H}$ & -5.0793 & 4.4847 & 4.4253 & 0.6349 & 2.2488 & -4.7082 & -10.6255 & -3.9119 & -3.3005 \\
\hline $\mathrm{H}$ & 1.1439 & 0.3464 & 6.5147 & -3.5062 & -4.0038 & -6.7317 & -6.7031 & 2.2077 & -5.4739 \\
\hline $\mathrm{H}$ & -5.1185 & -5.3639 & 2.7093 & -8.4432 & 0.9064 & -0.7362 & -7.0330 & 2.2432 & 3.7726 \\
\hline $\mathrm{H}$ & -3.3679 & -5.3001 & 2.9978 & -8.5731 & -0.5440 & -1.7524 & -6.1257 & 3.0369 & 2.4777 \\
\hline $\mathrm{H}$ & -4.4932 & -5.2697 & 4.3752 & -7.3303 & -0.4617 & -0.4769 & -7.8908 & 3.2955 & 2.6213 \\
\hline $\mathrm{H}$ & -6.6403 & -1.0515 & 3.8540 & -4.3508 & 2.8697 & -1.9863 & -9.4414 & -1.2013 & 1.6926 \\
\hline $\mathrm{H}$ & -5.6433 & -1.2516 & 2.4075 & -5.6019 & 2.8575 & -3.2604 & -7.6651 & -1.2713 & 1.5896 \\
\hline $\mathrm{H}$ & -2.3591 & -3.2749 & 3.6780 & -6.9349 & -0.9623 & -3.4336 & -6.5368 & 2.3657 & 0.1865 \\
\hline $\mathrm{H}$ & -3.4325 & -3.1185 & 5.0951 & -5.6451 & -0.9448 & -2.2028 & -8.3094 & 2.3767 & 0.2966 \\
\hline $\mathrm{H}$ & -5.8274 & -3.2013 & 4.7493 & -5.4834 & 1.1086 & -0.7499 & -9.3676 & 1.1989 & 2.1921 \\
\hline $\mathrm{H}$ & -6.4619 & -3.4178 & 3.0993 & -6.6220 & 2.4346 & -1.0636 & -8.3236 & 0.3086 & 3.3272 \\
\hline $\mathrm{H}$ & -3.3265 & 1.1229 & 7.2587 & -1.6498 & -1.6147 & -3.2275 & -10.5051 & 0.8142 & -3.1866 \\
\hline $\mathrm{H}$ & -4.1487 & -0.3318 & 6.6605 & -3.0351 & -0.9105 & -2.3834 & -10.1535 & 1.1547 & -1.4785 \\
\hline $\mathrm{H}$ & -3.0694 & -1.1308 & 2.8098 & -5.8260 & 0.8954 & -4.6271 & -6.5754 & -0.0876 & -0.1617 \\
\hline
\end{tabular}




\section{References}

(1) HyperChem(TM) Professional, version 8.0.10. 1115 NW 4th Street, Gainesville, Florida 32601, USA: Hypercube, Inc; 2011.

(2) Stewart, J. J. P. MOPAC2016, Stewart Computational Chemistry, 2016, http://OpenMOPAC.net (accessed October 15, 2019).

(3) Klamt, A.; Schüümann, G. COSMO: a New Approach to Dielectric Screening in Solvents with Explicit Expressions for the Screening Energy and Its Gradient. J. Chem. Soc., Perkin Trans. 2 1993, 799-805.

(4) Allouche, R. Gabedit 2.5.1, Gabedit - A Graphical User Interface for Computational Chemistry Softwares. J. Comput. Chem. 2011, 32, 174-182.

(5) Frisch, M. J.; Trucks, G. W.; Schlegel, H. B.; Scuseria, G. E.; Robb, M. A.; Cheeseman, J. R.; Scalmani, G.; Barone, V.; Mennucci, B.; Petersson, G. A., et al. Gaussian 09, Revision D.01; Gaussian, Inc.: Wallingford, CT, 2013.

(6) Becke, A. D. Density-Functional Thermochemistry. III. The Role of Exact Exchange. J. Chem. Phys. 1993, 98, 5648-5652.

(7) Grimme, S. Semiempirical GGA-Type Density Functional Constructed with a Long-Range Dispersion Correction. J. Comput. Chem. 2006, 27, 1787-1799.

(8) Tomasi, J.; Mennucci, B.; Cammi, R. Quantum Mechanical Continuum Solvation Models. Chem. Rev. 2005, 105, 2999-3093.

(9) Tantillo, D. J. Chemical Shift Repository, http://cheschirenmr.info/Instructions.htm (accessed November 21, 2015).

(10) Ignaczak, A.; Porwanski, S.; Szyszka, M. Deeper Insight into the Properties of the Newly Synthesized Macrocycles as Drug Receptors - Some Preliminary Quantum Chemical Studies. New J. Chem. 2017, 41, 521-529.

(11) Yamamoto, Y.; Onda, M.; Takahashi, Y.; Inoue, Y.; Chûjô, R. Two-Dimensional N.M.R. Spectra of $O$-Methylated Cyclomalto-Oligosaccharides: Assignment of ${ }^{1} \mathrm{H}$ and ${ }^{13} \mathrm{C}$ Resonances and Conformational Analysis. Carbohydr. Res. 1987, 170, 229-234.

(12) Correia, I.; Bezzenine, N.; Ronzani, N.; Platzer, N.; Beloeil, J.-C.; Doan, B.-T. Study of Inclusion Complexes of Acridine with $\beta$ - and (2,6-di- $O$-Methyl)- $\beta$-cyclodextrin by Use of Solubility Diagrams and NMR Spectroscopy. J. Phys. Org. Chem. 2002, 15, 647-459.

(13) Belica, S.; Jeziorska, D.; Urbaniak, P.; Buko, V. U.; Zavodnik, I. B.; Pałecz, B. Calorimetric and Spectroscopic Characterization of Complexes Between $\beta$-Cyclodextrin or Heptakis(2,6-di-Omethyl)- $\beta$-cyclodextrin and Sertraline Hydrochloride in Aqueous Solution. $J$. Chem. Thermodynamics 2014, 70, 160-167.

(14) Szejtli, J.; Lipták, A.; Jodál, I.; Fügedi, P.; Nánási, P.; Neszmélyi, A. Synthesis and ${ }^{13}$ C-NMR Spectroscopy of Methylated Beta-cyclodextrins. Starch-Stärke 1980, 32, 165-169. 